\title{
A desingularization of the main component of the moduli space of genus-one stable maps into $\mathbb{P}^{n}$
}

\author{
RAVI VAKIL \\ ALEKSEY ZiNGER
}

\begin{abstract}
We construct a natural smooth compactification of the space of smooth genus-one curves with $k$ distinct points in a projective space. It can be viewed as an analogue of a well-known smooth compactification of the space of smooth genus-zero curves, that is, the space of stable genus-zero maps $\overline{\mathfrak{M}}_{0, k}\left(\mathbb{P}^{n}, d\right)$. In fact, our compactification is obtained from the singular space of stable genus-one maps $\overline{\mathfrak{M}}_{1, k}\left(\mathbb{P}^{n}, d\right)$ through a natural sequence of blowups along "bad" subvarieties. While this construction is simple to describe, it requires more work to show that the end result is a smooth space. As a bonus, we obtain desingularizations of certain natural sheaves over the "main" irreducible component $\overline{\mathfrak{M}}_{1, k}^{0}\left(\mathbb{P}^{n}, d\right)$ of $\overline{\mathfrak{M}}_{1, k}\left(\mathbb{P}^{n}, d\right)$. A number of applications of these desingularizations in enumerative geometry and Gromov-Witten theory are described in the introduction, including the second author's proof of physicists' predictions for genus-one Gromov-Witten invariants of a quintic threefold.
\end{abstract}

14D20; 53D99

\section{Introduction}

\subsection{Background and applications}

The space of degree- $d$ genus- $g$ curves with $k$ distinct marked points in $\mathbb{P}^{n}$ is generally not compact, but admits a number of natural compactifications ${ }^{1}$. Among the most prominent compactifications is the moduli space of stable genus- $g$ maps, $\overline{\mathfrak{M}}_{g, k}\left(\mathbb{P}^{n}, d\right)$, constructed by Gromov [9] and Fulton-Pandharipande [6]. It has found numerous applications in classical enumerative geometry and is a central object in Gromov-Witten theory. However, most applications in enumerative geometry and some results in GWtheory have been restricted to the genus-zero case. The reason for this is essentially that the genus-zero moduli space has a particularly simple structure: it is smooth and contains the space of smooth genus-zero curves as a dense open subset. On the other

\footnotetext{
${ }^{1}$ We call a space $\overline{\mathfrak{M}}$ a compactification of $\mathfrak{M}$ if $\overline{\mathfrak{M}}$ is compact and contains $\mathfrak{M}$. In particular, $\mathfrak{M}$ need not be dense in $\overline{\mathfrak{M}}$.
} 
hand, the moduli spaces of positive-genus stable maps fail to satisfy either of these two properties. In fact, $\overline{\mathfrak{M}}_{g, k}\left(\mathbb{P}^{n}, d\right)$ can be arbitrarily singular according to Vakil [19]. It is thus natural to ask whether these failings can be remedied by modifying $\overline{\mathfrak{M}}_{g, k}\left(\mathbb{P}^{n}, d\right)$, preferably in a way that leads to a range of applications. As announced in [20] and shown in this paper, the answer is yes if $g=1$.

We denote by $\mathfrak{M}_{1, k}\left(\mathbb{P}^{n}, d\right)$ the subset of $\overline{\mathfrak{M}}_{1, k}\left(\mathbb{P}^{n}, d\right)$ consisting of the stable maps that have smooth domains. This space is smooth and contains the space of genusone curves with $k$ distinct marked points in $\mathbb{P}^{n}$ as a dense open subset, provided $d \geq 3$. However, $\mathfrak{M}_{1, k}\left(\mathbb{P}^{n}, d\right)$ is not compact. Let $\overline{\mathfrak{M}}_{1, k}^{0}\left(\mathbb{P}^{n}, d\right)$ be the closure of $\mathfrak{M}_{1, k}\left(\mathbb{P}^{n}, d\right)$ in the compact space $\overline{\mathfrak{M}}_{1, k}\left(\mathbb{P}^{n}, d\right)$. While $\overline{\mathfrak{M}}_{1, k}^{0}\left(\mathbb{P}^{n}, d\right)$ is not smooth, it turns out that a natural sequence of blowups along loci disjoint from $\mathfrak{M}_{1, k}\left(\mathbb{P}^{n}, d\right)$ leads to a desingularization of $\overline{\mathfrak{M}}_{1, k}^{0}\left(\mathbb{P}^{n}, d\right)$, which will be denoted by $\widetilde{\mathfrak{M}}_{1, k}^{0}\left(\mathbb{P}^{n}, d\right)$.

The situation is as good as one could possibly hope. A general strategy when attempting to desingularize some space is to blow up the "most degenerate" locus, then the proper transform of the "next most degenerate locus", and so on. This strategy works here, but with a novel twist: we apply it to the entire space of stable maps $\overline{\mathfrak{M}}_{1, k}\left(\mathbb{P}^{n}, d\right)$. The most degenerate locus is in fact an entire irreducible component, and blowing it up removes it ${ }^{2}$. Hence one by one we erase the "bad" components of $\overline{\mathfrak{M}}_{1, k}\left(\mathbb{P}^{n}, d\right)$. Each blowup of course changes the "good" component $\overline{\mathfrak{M}}_{1, k}^{0}\left(\mathbb{P}^{n}, d\right)$, and miraculously at the end of the process the resulting space $\widetilde{\mathfrak{M}}_{1, k}^{0}\left(\mathbb{P}^{n}, d\right)$ is nonsingular. We note that this cannot possibly be true for an arbitrary $g$, as $\mathfrak{M}_{g, k}\left(\mathbb{P}^{n}, d\right)$ behaves quite badly [19]. The sequential blowup construction itself is beautifully simple. It is completely described in the part of Section 1.2 ending with the main theorem of the paper, Theorem 1.1. However, showing that $\widetilde{\mathfrak{M}}_{1, k}^{0}\left(\mathbb{P}^{n}, d\right)$ is in fact smooth requires a considerable amount of preparation (which takes up Sections 2.1-4.2) and is finally completed in Section 4.3.

Since the smooth space $\widetilde{\mathfrak{M}}_{1, k}^{0}\left(\mathbb{P}^{n}, d\right)$ is obtained from $\overline{\mathfrak{M}}_{1, k}^{0}\left(\mathbb{P}^{n}, d\right)$ by blowing up along loci disjoint from $\mathfrak{M}_{1, k}\left(\mathbb{P}^{n}, d\right), \widetilde{\mathfrak{M}}_{1, k}^{0}\left(\mathbb{P}^{n}, d\right)$ is a smooth compactification of $\mathfrak{M}_{1, k}\left(\mathbb{P}^{n}, d\right)$. One would hope that there is a modular interpretation of this new compactification, and that one could then for example use this interpretation to construct the space $\widetilde{\mathfrak{M}}_{1, k}^{0}\left(\mathbb{P}^{n}, d\right)$ directly and show that it is smooth. Unfortunately, we have not managed to do this.

The desingularization $\widetilde{\mathfrak{M}}_{1, k}^{0}\left(\mathbb{P}^{n}, d\right)$ of $\overline{\mathfrak{M}}_{1, k}^{0}\left(\mathbb{P}^{n}, d\right)$ possesses a number of "good" properties and has a variety of applications to enumerative algebraic geometry and

\footnotetext{
${ }^{2}$ Blowing up an irreducible component of a stack will result in the component being removed (or "blown out of existence"), and the remainder of the stack is blown up along its intersection with the component in question.
} 
Gromov-Witten theory. It has already been observed by Fontanari [5] that the cohomology of $\widetilde{\mathfrak{M}}_{1, k}^{0}\left(\mathbb{P}^{n}, d\right)$ behaves in a certain respect like the cohomology of the moduli space of genus-one curves, $\overline{\mathcal{M}}_{1, k}$. The space $\widetilde{\mathfrak{M}}_{1, k}^{0}\left(\mathbb{P}^{n}, d\right)$ can be used to count genus-one curves in $\mathbb{P}^{n}$, mimicking the genus-zero results of Kontsevich-Manin [11] and Ruan-Tian [17] (though perhaps not their simple recursive formulas). Proceeding analogously to the genus-zero case (eg similar to Pandharipande [16], Vakil [18], and Zinger [23]), Theorem 1.1 can then be used to count genus-one curves with tangency conditions and singularities. In all cases, such counts can be expressed as integrals of natural cohomology classes on $\overline{\mathfrak{M}}_{1, k}^{0}\left(\mathbb{P}^{n}, d\right)$ or $\widetilde{\mathfrak{M}}_{1, k}^{0}\left(\mathbb{P}^{n}, d\right)$. Integrals on the latter space can be computed using the localization theorem of Atiyah-Bott [1], as $\widetilde{\mathfrak{M}}_{1, k}^{0}\left(\mathbb{P}^{n}, d\right)$ is smooth and inherits a torus action from $\mathbb{P}^{n}$ and $\overline{\mathfrak{M}}_{1, k}\left(\mathbb{P}^{n}, d\right)$.

We next discuss two types of applications of Theorem 1.1 in Gromov-Witten theory, as well as a bonus result of this paper, Theorem 1.2. It is shown by Zinger in [24] and [26] that the space $\overline{\mathfrak{M}}_{1, k}^{0}\left(\mathbb{P}^{n}, d\right)$ has a natural generalization to arbitrary almost Kähler manifolds and gives rise to new symplectic reduced genus-one $G W$-invariants. These reduced invariants are yet to be constructed in algebraic geometry. However, the spaces $\widetilde{\mathfrak{M}}_{1, k}^{0}\left(\mathbb{P}^{n}, d\right)$ do possess a number of "good" properties and give rise to algebraic invariants of algebraic manifolds; see the first and last sections of [20]. It is not clear whether these are the same as the reduced genus-one invariants, but it may be possible to verify this by using Theorem 1.2.

Theorem 1.1 also has applications to computing Gromov-Witten invariants of complete intersections, once it is combined with Theorem 1.2. Let $a$ be a nonnegative integer. For a general $s \in H^{0}\left(\mathbb{P}^{n}, \mathcal{O}_{\mathbb{P}^{n}}(a)\right)$,

$$
Y \equiv s^{-1}(0) \subset \mathbb{P}^{n}
$$

is a smooth hypersurface. We denote its degree- $d$ GW-invariant by $\operatorname{GW}_{g, k}^{Y}(d ; \cdot)$, ie

$$
\mathrm{GW}_{g, k}^{Y}(d ; \psi) \equiv\left\langle\psi,\left[\overline{\mathfrak{M}}_{g, k}(Y, d)\right]^{\mathrm{vir}}\right\rangle \quad \text { for all } \quad \psi \in H^{*}\left(\overline{\mathfrak{M}}_{g, k}(Y, d) ; \mathbb{Q}\right) .
$$

Suppose $\mathfrak{U}$ is the universal curve over $\overline{\mathfrak{M}}_{g, k}\left(\mathbb{P}^{n}, d\right)$, with structure map $\pi$ and evaluation map ev:

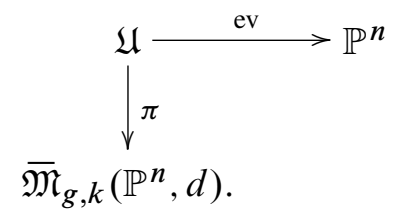

It can be shown that

$$
\operatorname{GW}_{0, k}^{Y}(d ; \psi)=\left\langle\psi \cdot e\left(\pi_{*} \mathrm{ev}^{*} \mathcal{O}_{\mathbb{P}^{n}}(a)\right),\left[\overline{\mathfrak{M}}_{0, k}\left(\mathbb{P}^{n}, d\right)\right]\right\rangle
$$


for all $\psi \in H^{*}\left(\overline{\mathfrak{M}}_{0, k}\left(\mathbb{P}^{n}, d\right) ; \mathbb{Q}\right)$; see Beauville [2] for example. The moduli space $\overline{\mathfrak{M}}_{0, k}\left(\mathbb{P}^{n}, d\right)$ is a smooth orbifold and

$$
\pi_{*} \operatorname{ev}^{*} \mathcal{O}_{\mathbb{P}^{n}}(a) \longrightarrow \overline{\mathfrak{M}}_{0, k}\left(\mathbb{P}^{n}, d\right)
$$

is a locally free sheaf, ie a vector bundle. The right-hand side of (1-1) can be computed via the classical localization theorem of Atiyah and Bott [1]. The complexity of this computation increases quickly with the degree $d$, but it has been completed in full generality in a number of different ways by Bertram [4], Gathmann [7], Givental [8], Lee [12] and Lian-Liu-Yau [14].

If $n=4$, so $Y$ is a threefold, then

$$
\begin{aligned}
\mathrm{GW}_{1, k}^{Y}(d ; \psi)=\frac{d(a-5)+2}{24} & \mathrm{GW}_{0, k}^{Y}(d ; \psi) \\
& +\left\langle\psi \cdot e\left(\pi_{*} \mathrm{ev}^{*} \mathcal{O}_{\mathbb{P}^{n}}(a)\right),\left[\overline{\mathfrak{M}}_{1, k}^{0}\left(\mathbb{P}^{4}, d\right)\right]\right\rangle
\end{aligned}
$$

for all primary $\psi \in H^{*}\left(\overline{\mathfrak{M}}_{1, k}\left(\mathbb{P}^{4}, d\right) ; \mathbb{Q}\right)$; see Li and Zinger [13, (1.5) and (1.12)]. This decomposition generalizes to arbitrary complete intersections $Y$ and perhaps even to higher-genus invariants. The sheaf

$$
\pi_{*} \operatorname{ev}^{*} \mathcal{O}_{\mathbb{P}^{n}}(a) \longrightarrow \overline{\mathfrak{M}}_{1, k}^{0}\left(\mathbb{P}^{4}, d\right)
$$

is not locally free. Nevertheless, its euler class is well-defined: the euler class of every desingularization of this sheaf is the same, in the sense of [25, Subsection 1.2]. This euler class can be geometrically interpreted as the zero set of a sufficiently good section of the cone

$$
\mathcal{V}_{1, k}^{d} \longrightarrow \overline{\mathfrak{M}}_{1, k}^{0}\left(\mathbb{P}^{4}, d\right)
$$

naturally associated to the sheaf $(1-3)^{3}$; see the second part of the next subsection and Lemma 5.1.

One would hope to compute the last expression in (1-2) by localization. However, since the variety $\overline{\mathfrak{M}}_{1, k}^{0}\left(\mathbb{P}^{4}, d\right)$ and the cone $\mathcal{V}_{1, k}^{d}$ are singular, the localization theorem of [1] is not immediately applicable in the given situation. Let

$$
\tilde{\pi}: \widetilde{\mathfrak{M}}_{1, k}^{0}\left(\mathbb{P}^{4}, d\right) \longrightarrow \overline{\mathfrak{M}}_{1, k}^{0}\left(\mathbb{P}^{4}, d\right)
$$

be the projection map. As a straightforward extension of the main desingularization construction of this paper, we show that the cone

$$
\tilde{\pi}^{*} \mathcal{V}_{1, k}^{d} \longrightarrow \widetilde{\mathfrak{M}}_{1, k}^{0}\left(\mathbb{P}^{4}, d\right)
$$

\footnotetext{
${ }^{3} \mathcal{V}_{1, k}^{d}$ is a variety such that the fibers of the projection map to $\overline{\mathfrak{M}}_{1, k}^{0}\left(\mathbb{P}^{4}, d\right)$ are vector spaces, but not necessarily of the same dimension.
} 
contains a vector bundle

$$
\widetilde{\mathcal{V}}_{1, k}^{d} \longrightarrow \widetilde{\mathfrak{M}}_{1, k}^{0}\left(\mathbb{P}^{4}, d\right)
$$

of rank $d a=\left.\operatorname{rk} \mathcal{V}_{1, k}^{d}\right|_{\mathfrak{M}_{1, k}^{0}\left(\mathbb{P}^{4}, d\right)}$; see Theorem 1.2. It then follows that

$$
\begin{aligned}
\left\langle\psi \cdot e\left(\pi_{*} \mathrm{ev}^{*} \mathcal{O}_{\mathbb{P}^{n}}(a)\right),\left[\overline{\mathfrak{M}}_{1, k}^{0}\left(\mathbb{P}^{4}, d\right)\right]\right\rangle & \equiv\left\langle\psi \cdot e\left(\mathcal{V}_{1, k}^{d}\right),\left[\overline{\mathfrak{M}}_{1, k}^{0}\left(\mathbb{P}^{4}, d\right)\right]\right\rangle \\
& =\left\langle\tilde{\pi}^{*} \psi \cdot e\left(\widetilde{\mathcal{V}}_{1, k}^{d}\right),\left[\widetilde{\mathfrak{M}}_{1, k}^{0}\left(\mathbb{P}^{4}, d\right)\right]\right\rangle .
\end{aligned}
$$

The last expression above is computable by localization. In fact, it is computed explicitly by the second author in [28], confirming the prediction of Bershadsky-Cecotti-OoguriVafa [3] for genus-one GW-invariants of a quintic threefold.

Remark Another approach to computing positive-genus GW-invariants has been proposed by Maulik-Pandharipande in [15]. In contrast to the approach of Li-Zinger in [13], it applies to arbitrary-genus invariants, but can at present be used to compute invariants of only low-dimensional and/or low-degree complete intersections.

The main desingularization construction of this paper is the subject of Section 4, but its key aspects are presented in the next subsection. The construction itself and its connections with Sections 2 and 3 are outlined in Section 1.3. We suggest that the reader return to Sections 1.2 and 1.3 before going through the technical details of the blowup constructions in Sections 2-4. In the next subsection, we also describe a natural sheaf over $\widetilde{\mathfrak{M}}_{1, k}^{0}\left(\mathbb{P}^{n}, d\right)$ which is closely related to the sheaf $\pi_{*} \operatorname{ev}^{*} \mathcal{O}_{\mathbb{P}^{n}}(a)$ over $\overline{\mathfrak{M}}_{1, k}^{0}\left(\mathbb{P}^{n}, d\right)$. It is shown to be locally free in Section 5. Finally, all the data necessary for applying the localization theorem of [1] to $\widetilde{\mathfrak{M}}_{1, k}^{0}\left(\mathbb{P}^{n}, d\right)$ and $e\left(\widetilde{\mathcal{V}}_{1, k}^{d}\right)$ is given in Section 1.4. In Appendix A, we list the most commonly used notation.

Throughout this article we work with Deligne-Mumford stacks. They can also be thought of as analytic orbivarieties. As we work with reduced scheme structures throughout the paper, we will call such objects simply varieties. Also, all immersions will be assumed to be from smooth varieties. ${ }^{4}$

The authors would like to thank Jun Li for many enlightening discussions and the referees for timely responses with many detailed suggestions. The first author was partially supported by the NSF grant DMS-0228011; the second author was partially supported by an NSF Postdoctoral Fellowship.

\footnotetext{
${ }^{4}$ The notion of "immersion" is often called "unramified" in algebraic geometry.
} 


\subsection{Description of the desingularization}

The moduli space $\overline{\mathfrak{M}}_{1, k}\left(\mathbb{P}^{n}, d\right)$ has irreducible components of various dimensions. One of these components is $\overline{\mathfrak{M}}_{1, k}^{0}\left(\mathbb{P}^{n}, d\right)$, the closure of the stratum $\mathfrak{M}_{1, k}\left(\mathbb{P}^{n}, d\right)$ of stable maps with smooth domains. We now describe natural subvarieties of $\overline{\mathfrak{M}}_{1, k}\left(\mathbb{P}^{n}, d\right)^{5}$ which contain the remaining components of $\overline{\mathfrak{M}}_{1, k}\left(\mathbb{P}^{n}, d\right)$. They will be indexed by the set

$$
\begin{gathered}
\mathcal{A}_{1}(d, k) \equiv\left\{\sigma=\left(m ; J_{P}, J_{B}\right): m \in \mathbb{Z}^{+}, m \leq d ;[k]=J_{P} \sqcup J_{B}\right\}, \\
\text { where } \quad[k]=\{1, \ldots, k\} .
\end{gathered}
$$

For each $\sigma \in \mathcal{A}_{1}(d, k)$, let $\mathfrak{M}_{1, \sigma}\left(\mathbb{P}^{n}, d\right)$ be the subset of $\overline{\mathfrak{M}}_{1, k}\left(\mathbb{P}^{n}, d\right)$ consisting of the stable maps $[\mathcal{C}, u]$ such that $\mathcal{C}$ is a smooth genus-one curve $E$ with $m$ smooth rational components attached directly to $E,\left.u\right|_{E}$ is constant, the restriction of $u$ to each rational component is nonconstant, and the marked points on $E$ are indexed by the set $J_{P}$. Here $P$ stands for "principal component", $B$ stands for "bubble component", and $\mathcal{A}$ stands for "admissible set". Figure 1 shows the domain of an element of $\mathfrak{M}_{1, \sigma}\left(\mathbb{P}^{n}, d\right)$, where $\sigma=(3 ;\{2\},\{1\})$, from the points of view of symplectic topology and of algebraic geometry. In the first diagram, each shaded disc represents a sphere; the integer next to each rational component $\mathcal{C}_{i}$ indicates the degree of $\left.u\right|_{\mathcal{C}_{i}}$. In the second diagram, the components of $\mathcal{C}$ are represented by curves, and the pair of integers next to each component $\mathcal{C}_{i}$ shows the genus of $\mathcal{C}_{i}$ and the degree of $\left.u\right|_{\mathcal{C}_{i}}$. In both diagrams, the marked points are labeled in bold face. Let $\overline{\mathfrak{M}}_{1, \sigma}\left(\mathbb{P}^{n}, d\right)$ be the closure of $\mathfrak{M}_{1, \sigma}\left(\mathbb{P}^{n}, d\right)$ in $\overline{\mathfrak{M}}_{1, k}\left(\mathbb{P}^{n}, d\right)$. The space $\overline{\mathfrak{M}}_{1, \sigma}\left(\mathbb{P}^{n}, d\right)$ has a number of irreducible components. These components are indexed by the splittings of the degree $d$ into $m$ positive integers and of the set $J_{B}$ into $m$ subsets. However, we do not need to distinguish these components.

It is straightforward to check that

$$
\overline{\mathfrak{M}}_{1, k}\left(\mathbb{P}^{n}, d\right)=\overline{\mathfrak{M}}_{1, k}^{0}\left(\mathbb{P}^{n}, d\right) \cup \underset{\sigma \in \mathcal{A}_{1}(d, k)}{\bigcup} \overline{\mathfrak{M}}_{1, \sigma}\left(\mathbb{P}^{n}, d\right) .
$$

Dimensional considerations imply that if $\sigma=\left(m ; J_{P}, J_{B}\right) \in A_{1}(d, k)$ and $m \leq n$, then $\overline{\mathfrak{M}}_{1, \sigma}\left(\mathbb{P}^{n}, d\right)$ is a union of components of $\overline{\mathfrak{M}}_{1, k}\left(\mathbb{P}^{n}, d\right)$. The converse holds as well: $\overline{\mathfrak{M}}_{1, \sigma}\left(\mathbb{P}^{n}, d\right)$ is contained in $\overline{\mathfrak{M}}_{1, k}^{0}\left(\mathbb{P}^{n}, d\right)$ if $m>n$ by [24, Theorem 2.3]. However, we will use the entire collection $\mathcal{A}_{1}(d, k)$ of subvarieties of $\overline{\mathfrak{M}}_{1, k}\left(\mathbb{P}^{n}, d\right)$ to construct $\widetilde{\mathfrak{M}}_{1, k}^{0}\left(\mathbb{P}^{n}, d\right)$. The independence of the indexing set $\mathcal{A}_{1}(d, k)$ of $n$ leads to a number

\footnotetext{
${ }^{5}$ In fact, these will be substacks of the stack $\overline{\mathfrak{M}}_{1, k}\left(\mathbb{P}^{n}, d\right)$. They can also be thought of as analytic sub-orbivarieties of the analytic orbivariety $\overline{\mathfrak{M}}_{1, k}\left(\mathbb{P}^{n}, d\right)$. As we work with reduced scheme structures throughout the paper, we will call such objects simply varieties.
} 
of good properties being satisfied by our blowup construction; see (2) of Theorem 1.1 and the second part of this subsection. It may also be possible to use this construction to define reduced genus-one GW-invariants in algebraic geometry; this is achieved in symplectic topology by the second author in [26].
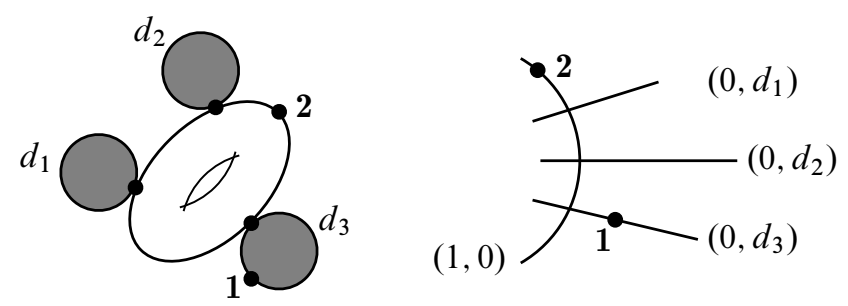

$$
\begin{aligned}
& \sigma=(3 ;\{2\},\{1\}) \\
& d_{1}+d_{2}+d_{3}=d \\
& d_{1}, d_{2}, d_{3}>0
\end{aligned}
$$

Figure 1: The domain of an element of $\mathfrak{M}_{1, \sigma}\left(\mathbb{P}^{n}, d\right)$

We define a partial ordering $\prec$ on the set $\mathcal{A}_{1}(d, k)$ by

$$
\sigma^{\prime} \equiv\left(m^{\prime} ; J_{P}^{\prime}, J_{B}^{\prime}\right) \prec \sigma \equiv\left(m ; J_{P}, J_{B}\right) \quad \text { if } \quad \sigma^{\prime} \neq \sigma, m^{\prime} \leq m \text {, and } J_{P}^{\prime} \subseteq J_{P} .
$$

This relation is illustrated in Figure 2, where an element $\sigma$ of $\mathcal{A}_{1}(d, k)$ is represented by an element of the corresponding space $\mathfrak{M}_{1, \sigma}\left(\mathbb{P}^{n}, d\right)$. We indicate that the degree of the stable map on every bubble component is positive by shading the disks in the figure. We show only the marked points lying on the principal component. The exact distribution of the remaining marked points between the components is irrelevant.
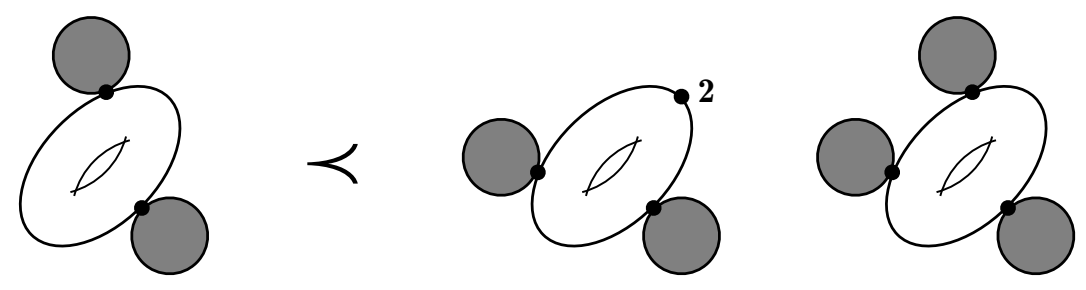

Figure 2: Examples of partial ordering (1-5)

Choose an ordering $<$ on $\mathcal{A}_{1}(d, k)$ extending the partial ordering $\prec$. The desingularization

$$
\tilde{\pi}: \widetilde{\mathfrak{M}}_{1, k}^{0}\left(\mathbb{P}^{n}, d\right) \longrightarrow \overline{\mathfrak{M}}_{1, k}^{0}\left(\mathbb{P}^{n}, d\right)
$$

is constructed by blowing up $\overline{\mathfrak{M}}_{1, k}\left(\mathbb{P}^{n}, d\right)$ along the subvarieties $\overline{\mathfrak{M}}_{1, \sigma}\left(\mathbb{P}^{n}, d\right)$ and their proper transforms in the order specified by $<$. In other words, we first blow up $\overline{\mathfrak{M}}_{1, k}\left(\mathbb{P}^{n}, d\right)$ along $\overline{\mathfrak{M}}_{1, \sigma_{\text {min }}}\left(\mathbb{P}^{n}, d\right)$, where

$$
\sigma_{\min } \equiv(1 ; \varnothing,[k])
$$


is the smallest element of $\mathcal{A}_{1}(d, k)$. We then blow up the resulting space along the proper transform of $\overline{\mathfrak{M}}_{1, \sigma_{2}}\left(\mathbb{P}^{n}, d\right)$, where $\sigma_{2}$ is the smallest element of $\mathcal{A}_{1}(d, k)-$ $\left\{\sigma_{\min }\right\}$. We continue this procedure until we blow up along the proper transform of $\overline{\mathfrak{M}}_{1, \sigma_{\max }}\left(\mathbb{P}^{n}, d\right)$, where

$$
\sigma_{\max }=(d ;[k], \varnothing)
$$

is the largest element of $\mathcal{A}_{1}(d, k)$. The variety resulting from this last blowup is the proper transform $\widetilde{\mathfrak{M}}_{1, k}^{0}\left(\mathbb{P}^{n}, d\right)$ of $\overline{\mathfrak{M}}_{1, k}^{0}\left(\mathbb{P}^{n}, d\right)$, as all other irreducible components of $\overline{\mathfrak{M}}_{1, k}\left(\mathbb{P}^{n}, d\right)$ have been "blown out of existence".

We describe the first interesting case of this construction, ie for $\overline{\mathfrak{M}}_{1,0}^{0}\left(\mathbb{P}^{2}, 3\right)$, in detail in [20]. The space $\widetilde{\mathfrak{M}}_{1,0}^{0}\left(\mathbb{P}^{2}, 3\right)$ is a smooth compactification of the space of smooth plane cubics. It has a richer structure than the naive compactification, $\mathbb{P}^{9}$, does.

Theorem 1.1 Suppose $n, d \in \mathbb{Z}^{+}, k \in \overline{\mathbb{Z}}^{+},<$is an ordering on the set $\mathcal{A}_{1}(d, k)$ extending the partial ordering $\prec$, and

$$
\tilde{\pi}: \widetilde{\mathfrak{M}}_{1, k}^{0}\left(\mathbb{P}^{n}, d\right) \longrightarrow \overline{\mathfrak{M}}_{1, k}^{0}\left(\mathbb{P}^{n}, d\right)
$$

is the blowup of $\overline{\mathfrak{M}}_{1, k}^{0}\left(\mathbb{P}^{n}, d\right)$ obtained by blowing up $\overline{\mathfrak{M}}_{1, k}\left(\mathbb{P}^{n}, d\right)$ along the subvarieties $\overline{\mathfrak{M}}_{1, \sigma}\left(\mathbb{P}^{n}, d\right)$ and their proper transforms in the order specified by $<$.

(1) The variety $\widetilde{\mathfrak{M}}_{1, k}^{0}\left(\mathbb{P}^{n}, d\right)$ is smooth and is independent of the choice of ordering < extending $\prec$.

(2) For all $m \leq n$, the embedding $\overline{\mathfrak{M}}_{1, k}^{0}\left(\mathbb{P}^{m}, d\right) \longrightarrow \overline{\mathfrak{M}}_{1, k}^{0}\left(\mathbb{P}^{n}, d\right)$ lifts to an embedding

$$
\widetilde{\mathfrak{M}}_{1, k}^{0}\left(\mathbb{P}^{m}, d\right) \longrightarrow \widetilde{\mathfrak{M}}_{1, k}^{0}\left(\mathbb{P}^{n}, d\right)
$$

and the image of the latter embedding is the preimage of $\overline{\mathfrak{M}}_{1, k}^{0}\left(\mathbb{P}^{m}, d\right)$ under $\tilde{\pi}$.

(3) The blowup locus at every step of the blowup construction is a smooth subvariety in the corresponding blowup of $\overline{\mathfrak{M}}_{1, k}\left(\mathbb{P}^{n}, d\right)$.

(4) All fibers of $\tilde{\pi}$ are connected.

Remark While in Section 4 we analyze the blowup construction starting with the reduced scheme structure on $\overline{\mathfrak{M}}_{1, k}\left(\mathbb{P}^{n}, d\right)$, Theorem 1.1 applies to the standard scheme structure on $\overline{\mathfrak{M}}_{1, k}\left(\mathbb{P}^{n}, d\right)$ as well. It is known that the space

$$
\mathfrak{M}_{1, k}^{\mathrm{eff}}\left(\mathbb{P}^{n}, d\right) \equiv \overline{\mathfrak{M}}_{1, k}^{0}\left(\mathbb{P}^{n}, d\right)-\underset{\sigma \in \mathcal{A}_{1}(d, k)}{\bigcup} \overline{\mathfrak{M}}_{1, \sigma}\left(\mathbb{P}^{n}, d\right),
$$

consisting of stable maps with no contracted genus-one component, is a smooth stack (as such maps are unobstructed; see for example Proposition 5.5(c) of [18]). Thus, its 
scheme-theoretic closure, $\overline{\mathfrak{M}}_{1, k}^{0}\left(\mathbb{P}^{n}, d\right)$, is reduced. During the blowup process all other components of $\overline{\mathfrak{M}}_{1, k}\left(\mathbb{P}^{n}, d\right)$ are "blown out of existence", as is any nonreduced scheme structure.

In Theorem 1.1 and throughout the rest of the paper we denote by $\overline{\mathbb{Z}}^{+}$the set of nonnegative integers. We analyze the sequential blowup construction of Theorem 1.1 in Section 4 using the inductive assumptions $(I 1)-(I 15)$ of Section 4.3. One of these assumptions, (I3), implies the second part of the first statement of Theorem 1.1, as different choices of an ordering $<$ extending the partial ordering $\prec$ correspond to different orders of blowups along subvarieties that are disjoint. For example, suppose

$$
\sigma_{\varnothing}=(2 ; \varnothing,\{1,2\}), \quad \sigma_{1}=(2 ;\{1\},\{2\}), \quad \text { and } \quad \sigma_{2}=(2 ;\{2\},\{1\}) .
$$

While $\overline{\mathfrak{M}}_{1, \sigma_{1}}\left(\mathbb{P}^{n}, d\right)$ and $\overline{\mathfrak{M}}_{1, \sigma_{2}}\left(\mathbb{P}^{n}, d\right)$ do intersect in $\overline{\mathfrak{M}}_{1,2}\left(\mathbb{P}^{n}, d\right)$, their proper transforms are disjoint after the blowup along $\overline{\mathfrak{M}}_{1, \sigma_{\varnothing}}\left(\mathbb{P}^{n}, d\right)$. The second statement of Theorem 1.1 follows immediately from the description of the blowup construction in this and the next subsections, as each step of the construction commutes with the embeddings of the moduli spaces induced by the embedding $\mathbb{P}^{m} \longrightarrow \mathbb{P}^{n}$.

The main claim of this paper is that $\widetilde{\mathfrak{M}}_{1, k}^{0}\left(\mathbb{P}^{n}, d\right)$ is a smooth variety. The structure of $\mathfrak{M}_{1, k}^{\text {eff }}\left(\mathbb{P}^{n}, d\right)$ is well understood; see [18, Prop. 5.5(c)] for example. In particular, $\mathfrak{M}_{1, k}^{\text {eff }}\left(\mathbb{P}^{n}, d\right)$ is smooth. Below we describe the structure of the complement $\partial \widetilde{\mathfrak{M}}_{1, k}^{0}\left(\mathbb{P}^{n}, d\right)$ of $\mathfrak{M}_{1, k}^{\text {eff }}\left(\mathbb{P}^{n}, d\right)$ in $\widetilde{\mathfrak{M}}_{1, k}^{0}\left(\mathbb{P}^{n}, d\right)$.

If $J$ is a finite set and $g$ is a nonnegative integer, we denote by $\overline{\mathcal{M}}_{g, J}$ the moduli space of stable genus $-g$ curves with $|J|$ marked points, which are indexed by the set $J$. Similarly, we denote by $\overline{\mathfrak{M}}_{g, J}\left(\mathbb{P}^{n}, d\right)$ the moduli space of stable maps from genus- $g$ curves with marked points indexed by $J$ to $\mathbb{P}^{n}$. If $j \in J$, let

$$
\mathrm{ev}_{j}: \overline{\mathfrak{M}}_{g, J}\left(\mathbb{P}^{n}, d\right) \longrightarrow \mathbb{P}^{n}
$$

be the evaluation map at the marked point labeled by $j$.

If $\sigma=\left(m ; J_{P}, J_{B}\right)$ is an element of $\mathcal{A}_{1}(d, k)$, we define

$$
\begin{aligned}
& \overline{\mathcal{M}}_{\sigma ; P}=\overline{\mathcal{M}}_{1,[m] \sqcup J_{P}}, \\
& \overline{\mathfrak{M}}_{\sigma ; B}\left(\mathbb{P}^{n}, d\right)=\left\{\left(b_{1}, \ldots, b_{m}\right) \in \prod_{i=1}^{m} \overline{\mathfrak{M}}_{0,\{0\} \sqcup J_{i}}\left(\mathbb{P}^{n}, d_{i}\right): d_{i}>0, \sum_{i=1}^{m} d_{i}=d ;\right. \\
&\left.\bigsqcup_{i=1}^{m} J_{i}=J_{B} ; \operatorname{ev}_{0}\left(b_{i_{1}}\right)=\operatorname{ev}_{0}\left(b_{i_{2}}\right) \forall i_{1}, i_{2} \in[m]\right\} .
\end{aligned}
$$


There is a natural node-identifying surjective immersion

$$
\iota_{\sigma}: \overline{\mathcal{M}}_{\sigma ; P} \times \overline{\mathfrak{M}}_{\sigma ; B}\left(\mathbb{P}^{n}, d\right) \longrightarrow \overline{\mathfrak{M}}_{1, \sigma}\left(\mathbb{P}^{n}, d\right) \subset \overline{\mathfrak{M}}_{1, k}\left(\mathbb{P}^{n}, d\right) .
$$

As before, $P$ denotes "principal component", and $B$ denotes "bubble components". This immersion descends to the quotient

$$
\bar{\iota}_{\sigma}:\left(\overline{\mathcal{M}}_{\sigma ; P} \times \overline{\mathfrak{M}}_{\sigma ; B}\left(\mathbb{P}^{n}, d\right)\right) / G_{\sigma} \longrightarrow \overline{\mathfrak{M}}_{1, \sigma}\left(\mathbb{P}^{n}, d\right),
$$

where $G_{\sigma} \equiv S_{m}$ is the symmetric group on $m$ elements. If $m \geq 3, \bar{\iota}_{\sigma}$ is not an isomorphism as some subvarieties of the left side are identified. An example of a point on the right which is the image of two points on the left is given in Figure 3. In addition to the conventions used in Figure 1, in the first, symplectic-topology, diagram of Figure 3 we leave the components of the domain on which the map is constant unshaded. The subvarieties identified by the map $\bar{\iota}_{\sigma}$ get "unidentified" after taking the proper transform of $\overline{\mathfrak{M}}_{1, \sigma}\left(\mathbb{P}^{n}, d\right)$ in the blowup of $\overline{\mathfrak{M}}_{1, k}\left(\mathbb{P}^{n}, d\right)$ at the step corresponding to

$$
\tilde{\sigma}=\max \left\{\sigma^{\prime} \in \mathcal{A}_{1}(d, k): \sigma^{\prime} \prec \sigma\right\} .
$$

This is insured by the inductive assumption (I13) in Section 4.3 and implies the third statement of Theorem 1.1. For example, if $m=3$ and $k=0$ as in Figure 3, the "identified" subvarieties are "unidentified" after the blowup of the proper transform of $\overline{\mathfrak{M}}_{1,(2 ; \varnothing, \varnothing)}\left(\mathbb{P}^{n}, d\right)$.

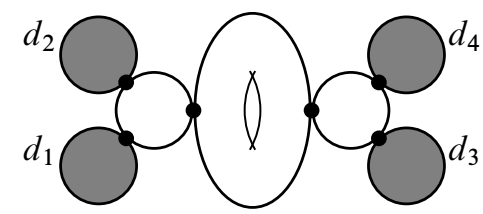

$\sigma=(3 ; \emptyset, \emptyset), \quad d_{1}+d_{2}+d_{3}+d_{4}=d, \quad d_{1}, d_{2}, d_{3}, d_{4}>0$

Figure 3: A point in $\overline{\mathfrak{M}}_{1, \sigma}\left(\mathbb{P}^{n}, d\right) \subset \overline{\mathfrak{M}}_{1,0}\left(\mathbb{P}^{n}, d\right)$ with two preimages under $\bar{\iota}_{\sigma}$

Remark Throughout the paper, we use $\mathfrak{M}$ (fraktur font) to denote moduli spaces of stable maps, of genus zero or one, into $\mathbb{P}^{n}$. We use $\mathcal{M}$ (calligraphic font) to denote moduli spaces of stable curves.

For each $i \in[m]$, let

$$
\pi_{i}: \overline{\mathfrak{M}}_{\sigma ; B}\left(\mathbb{P}^{n}, d\right) \longrightarrow \bigsqcup_{d_{i}>0, J_{i} \subset J_{B}} \overline{\mathfrak{M}}_{0,\{0\} \sqcup J_{i}}\left(\mathbb{P}^{n}, d_{i}\right)
$$


be the natural projection onto the $i$-th component. We put

$$
F_{\sigma ; B}=\bigoplus_{i=1}^{m} \pi_{i}^{*} L_{0},
$$

where $L_{0} \longrightarrow \overline{\mathfrak{M}}_{0,\{0\} \sqcup J_{i}}\left(\mathbb{P}^{n}, d_{i}\right)$ is the universal tangent line bundle for the marked point 0 . In Sections 2.3 and 3.4, we construct blowups

$$
\tilde{\pi}_{\sigma ; P} \equiv \pi_{1,\left([m], J_{P}\right)}: \widetilde{\mathcal{M}}_{\sigma ; P} \equiv \widetilde{\mathcal{M}}_{1,\left([m], J_{P}\right)} \longrightarrow \overline{\mathcal{M}}_{\sigma ; P} \equiv \overline{\mathcal{M}}_{1,[m] \sqcup J_{P}}
$$

and

$$
\tilde{\pi}_{\sigma ; B} \equiv \pi_{0,\left([m], J_{B}\right)}: \widetilde{\mathfrak{M}}_{\sigma ; B}\left(\mathbb{P}^{n}, d\right) \equiv \widetilde{\mathfrak{M}}_{0,\left([m], J_{B}\right)}\left(\mathbb{P}^{n}, d\right) \longrightarrow \mathbb{P} F_{\sigma ; B} \equiv \mathbb{P} F_{\left([m], J_{B}\right)} .
$$

We also construct a section

$$
\widetilde{\mathcal{D}}_{\sigma ; B} \equiv \widetilde{\mathcal{D}}_{\left([m], J_{B}\right)} \in \Gamma\left(\widetilde{\mathfrak{M}}_{\sigma ; B}\left(\mathbb{P}^{n}, d\right) ; \mathbb{E}_{\sigma ; B}^{*} \otimes \tilde{\pi}_{\sigma ; B}^{*} \pi_{\mathbb{P} F_{\sigma ; B}}^{*} \mathrm{ev}_{0}^{*} T \mathbb{P}^{n}\right),
$$

where $\quad \mathrm{ev}_{0}: \overline{\mathfrak{M}}_{\sigma ; B}\left(\mathbb{P}^{n}, d\right) \longrightarrow \mathbb{P}^{n} \quad$ and $\quad \pi_{\mathbb{P} F_{\sigma ; B}}: \mathbb{P} F_{\sigma ; B} \longrightarrow \overline{\mathfrak{M}}_{\sigma ; B}\left(\mathbb{P}^{n}, d\right)$ are the natural evaluation map and the bundle projection map, respectively, and

$$
\mathbb{E}_{\sigma ; B} \equiv \widetilde{\mathbb{E}} \longrightarrow \widetilde{\mathfrak{M}}_{\sigma ; B}\left(\mathbb{P}^{n}, d\right) \equiv \widetilde{\mathfrak{M}}_{0,\left([m], J_{B}\right)}\left(\mathbb{P}^{n}, d\right)
$$

is a line bundle. This line bundle is the sum of the tautological line bundle

$$
\gamma_{\sigma ; B} \longrightarrow \mathbb{P} F_{\sigma ; B}
$$

and all exceptional divisors. The section $\widetilde{\mathcal{D}}_{\sigma ; B}$ is transverse to the zero section. Thus, its zero set,

$$
\widetilde{\mathcal{Z}}_{\sigma ; B}\left(\mathbb{P}^{n}, d\right) \equiv \widetilde{\mathcal{D}}_{\sigma ; B}^{-1}(0) \subset \widetilde{\mathfrak{M}}_{\sigma ; B}\left(\mathbb{P}^{n}, d\right),
$$

is a smooth subvariety. The boundary $\partial \widetilde{\mathfrak{M}}_{1, k}^{0}\left(\mathbb{P}^{n}, d\right)$ of $\widetilde{\mathfrak{M}}_{1, k}^{0}\left(\mathbb{P}^{n}, d\right)$ is a union of smooth divisors:

$\partial \widetilde{\mathfrak{M}}_{1, k}^{0}\left(\mathbb{P}^{n}, d\right)=\bigcup_{\sigma \in \mathcal{A}_{1}(d, k)} \widetilde{\mathcal{Z}}_{\sigma}\left(\mathbb{P}^{n}, d\right) / G_{\sigma}$, where $\widetilde{\mathcal{Z}}_{\sigma}\left(\mathbb{P}^{n}, d\right)=\widetilde{\mathcal{M}}_{\sigma ; P} \times \widetilde{\mathcal{Z}}_{\sigma ; B}\left(\mathbb{P}^{n}, d\right) ;$

see the inductive assumptions (I7) and (I8) in Section 4.3 and Figure 4. By the inductive assumption (I6) and (I7), the normal bundle of $\widetilde{\mathcal{Z}}_{\sigma}\left(\mathbb{P}^{n}, d\right)$ in $\widetilde{\mathfrak{M}}_{1, k}\left(\mathbb{P}^{n}, d\right)$ is the quotient of the line bundle

$$
\mathbb{L}_{\sigma ; P} \otimes \tilde{\pi}_{\sigma ; B}^{*} \gamma_{\sigma ; B} \longrightarrow \widetilde{\mathcal{M}}_{\sigma ; P} \times \widetilde{\mathcal{Z}}_{\sigma ; B}\left(\mathbb{P}^{n}, d\right)
$$

by the $G_{\sigma}$-action, where

$$
\mathbb{L}_{\sigma ; P} \equiv \mathbb{L} \longrightarrow \widetilde{\mathcal{M}}_{\sigma ; P} \equiv \widetilde{\mathcal{M}}_{1,\left([m], J_{P}\right)}
$$


is the universal tangent line bundle constructed in Section 2.3. Thus we conclude that $\widetilde{\mathfrak{M}}_{1, k}^{0}\left(\mathbb{P}^{n}, d\right)$ is smooth, as the open subset $\mathfrak{M}_{1, k}^{\text {eff }}\left(\mathbb{P}^{n}, d\right)$ is smooth, and its complement is a union of smooth divisors whose normal sheaves are line bundles (ie with their reduced induced scheme structure, they are Cartier divisors).

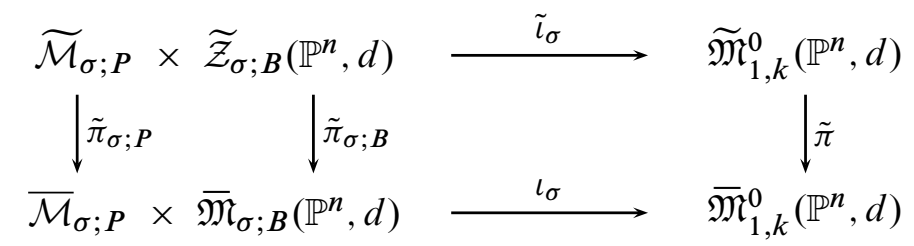

Figure 4: Changes in the boundary structure of $\overline{\mathfrak{M}}_{1, k}^{0}\left(\mathbb{P}^{n}, d\right)$ under the desingularization

Remark 1 In the Gromov-Witten theory, the symbol $\mathbb{E}$ is commonly used to denote the Hodge vector bundle of holomorphic differentials. It is the zero vector bundle in the genus-zero case. The line bundles over moduli spaces of genus-zero curves and maps we denote by $\mathbb{E}$, with various decorations, play roles analogous to that of the Hodge line bundle over moduli spaces of genus-one curves. The most overt parallel is described at the end of Section 2.2. There are deeper, more subtle, connections as well; compare the structural descriptions of Lemmas 3.8 and 4.10, for example.

Remark 2 Throughout this paper, the symbols $\mathcal{D}$ and $\mathfrak{D}$, with various decorations, denote vector bundle sections related to derivatives of holomorphic maps into $\mathbb{P}^{n}$ and of holomorphic bundle sections. In most cases, such bundle sections are viewed as vector bundle homomorphisms.

The final claim of Theorem 1.1 follows from the fact that $\overline{\mathfrak{M}}_{1, k}^{0}\left(\mathbb{P}^{n}, d\right)$ is unibranch (locally irreducible). If $\pi: Y \longrightarrow X$ is a surjective birational map of irreducible varieties, and $\pi^{-1}(x)$ is not connected for some $x \in X$, then $X$ is not unibranch at $x$.

We next describe a desingularization of the sheaf $\pi_{*} \mathrm{ev}^{*} \mathcal{O}_{\mathbb{P}^{n}}(a)$ and of the corresponding cone $\mathcal{V}_{1, k}^{d}$ over $\overline{\mathfrak{M}}_{1, k}^{0}\left(\mathbb{P}^{n}, d\right)$. Let $\widetilde{\mathfrak{U}}=\widetilde{\pi}^{*} \mathfrak{U}$ be the pullback of $\mathfrak{U}$ by $\tilde{\pi}$ :

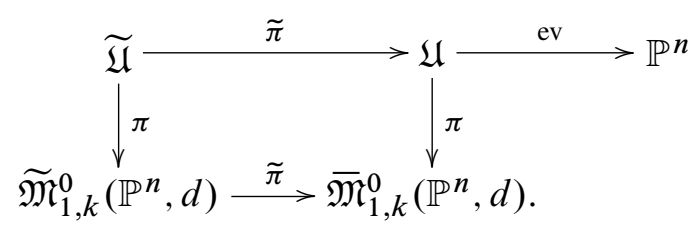

For each $\sigma \in \mathcal{A}_{1}(d, k)$, let

$$
\mathcal{V}_{\sigma ; B} \longrightarrow \overline{\mathfrak{M}}_{\sigma ; B}\left(\mathbb{P}^{n}, d\right)
$$


be the cone induced by the sheaf $\mathcal{O}_{\mathbb{P}^{n}}(a)$, similarly to $\mathcal{V}_{g, k}^{d}$; see Section 5.2 for details. It is a vector bundle of rank $d a+1$. We note that

$$
\left.\tilde{\pi}^{*} \mathcal{V}_{1, k}^{d}\right|_{\widetilde{\mathcal{Z}}_{\sigma}\left(\mathbb{P}^{n}, d\right)}=\pi_{B}^{*}\left\{\left.\tilde{\pi}_{\sigma ; B}^{*} \pi_{\mathbb{P} F_{\sigma ; B}}^{*} \mathcal{V}_{\sigma ; B}\right|_{\widetilde{\mathcal{Z}}_{\sigma ; B}\left(\mathbb{P}^{n}, d\right)}\right\} / G_{\sigma}
$$

where $\quad \pi_{B}: \widetilde{\mathcal{M}}_{\sigma ; P} \times \widetilde{\mathcal{Z}}_{\sigma ; B}\left(\mathbb{P}^{n}, d\right) \longrightarrow \widetilde{\mathcal{Z}}_{\sigma ; B}\left(\mathbb{P}^{n}, d\right)$

is the projection map. Let $\mathcal{L}=\gamma^{* \otimes a}$, where $\gamma \longrightarrow \mathbb{P}^{n}$ is the tautological line bundle.

Theorem 1.2 Suppose $d, n, a \in \mathbb{Z}^{+}$and $k \in \overline{\mathbb{Z}}^{+}$.

(1) The sheaf $\pi_{*} \tilde{\pi}^{*} \operatorname{ev}^{*} \mathcal{O}_{\mathbb{P}^{n}}(a)$ over $\widetilde{\mathfrak{M}}_{1, k}^{0}\left(\mathbb{P}^{n}, d\right)$ is locally free and of the expected rank, ie $d a$.

(2) If $\widetilde{\mathcal{V}}_{1, k}^{d} \subset \tilde{\pi}^{*} \mathcal{V}_{1, k}^{d}$ is the corresponding vector bundle and $\sigma \in \mathcal{A}_{1}(d, k)$, then there exists a surjective bundle homomorphism

$$
\widetilde{\mathfrak{D}}_{\sigma ; B}:\left.\tilde{\pi}_{\sigma ; B}^{*} \pi_{\mathbb{P} F_{\sigma ; B}}^{*} \mathcal{V}_{\sigma ; B}\right|_{\widetilde{\mathcal{Z}}_{\sigma ; B}\left(\mathbb{P}^{n}, d\right)} \longrightarrow \mathbb{E}_{\sigma ; B}^{*} \otimes \tilde{\pi}_{\sigma ; B}^{*} \pi_{\mathbb{P} F_{\sigma ; B}}^{*} \mathrm{ev}_{0}^{*} \mathcal{L}
$$

over $\widetilde{\mathcal{Z}}_{\sigma ; B}\left(\mathbb{P}^{n}, d\right)$ such that

$$
\left.\widetilde{\mathcal{V}}_{1, k}^{d}\right|_{\widetilde{\mathcal{Z}}_{\sigma}\left(\mathbb{P}^{n}, d\right)}=\left(\pi_{B}^{*} \operatorname{ker} \widetilde{\mathfrak{D}}_{\sigma ; B}\right) / G_{\sigma}
$$

(3) $\tilde{\pi}_{*} \pi_{*} \tilde{\pi}^{*} \operatorname{ev}^{*} \mathcal{O}_{\mathbb{P}^{n}}(a)=\pi_{*} \operatorname{ev}^{*} \mathcal{O}_{\mathbb{P}^{n}}(a)$ over $\overline{\mathfrak{M}}_{1, k}^{0}\left(\mathbb{P}^{n}, d\right)$.

The first two statements of this theorem can be used to compute expressions like (1-4) via the classical localization theorem and the short exact sequence (1-10) below. We prove them by working with the cone

$$
p: \overline{\mathfrak{M}}_{1, k}(\mathcal{L}, d) \longrightarrow \overline{\mathfrak{M}}_{1, k}\left(\mathbb{P}^{n}, d\right) .
$$

The sheaves $\pi_{*} \operatorname{ev}^{*} \mathcal{O}_{\mathbb{P}^{n}}(a)$ and $\pi_{*} \tilde{\pi}^{*} \mathrm{ev}^{*} \mathcal{O}_{\mathbb{P}^{n}}(a)$ are the sheaves of (holomorphic) sections of

$$
\left.\mathcal{V}_{1, k}^{d} \equiv \overline{\mathfrak{M}}_{1, k}(\mathcal{L}, d)\right|_{\overline{\mathfrak{M}}_{1, k}^{0}\left(\mathbb{P}^{n}, d\right)} \longrightarrow \overline{\mathfrak{M}}_{1, k}^{0}\left(\mathbb{P}^{n}, d\right)
$$

and $\tilde{\pi}^{*} \mathcal{V}_{1, k}^{d}$, respectively; see Lemma 5.1. In Section 5.4, we lift the blowup construction of Section 3.4 to $\overline{\mathfrak{M}}_{1, k}(\mathcal{L}, d)$. In particular, we blow up $\overline{\mathfrak{M}}_{1, k}(\mathcal{L}, d)$ along the subvarieties

$$
\overline{\mathfrak{M}}_{1, \sigma}(\mathcal{L}, d)=p^{-1}\left(\overline{\mathfrak{M}}_{1, \sigma}\left(\mathbb{P}^{n}, d\right)\right), \quad \sigma \in \mathcal{A}_{1}(d, k),
$$

and their proper transforms. The end result of this construction, which we denote by $\widetilde{\mathfrak{M}}_{1, k}^{0}(\mathcal{L}, d)$, is smooth for essentially the same reasons that $\overline{\mathfrak{M}}_{1, k}^{0}\left(\mathbb{P}^{n}, d\right)$ is. The only additional input we need is Lemma 5.7, which is a restatement of the key result 
concerning the structure of the cone $\mathcal{V}_{1, k}^{d}$ obtained by the second author in [25]. The bundle

$$
\tilde{p}: \widetilde{\mathfrak{M}}_{1, k}^{0}(\mathcal{L}, d) \longrightarrow \widetilde{\mathfrak{M}}_{1, k}^{0}\left(\mathbb{P}^{n}, d\right)
$$

of vector spaces of the same rank contains $\widetilde{\mathfrak{M}}_{1, k}^{0}\left(\mathbb{P}^{n}, d\right)$ as the zero section. Thus, $\tilde{p}$ is a vector bundle. There is a natural inclusion

$$
\widetilde{\mathfrak{M}}_{1, k}^{0}(\mathcal{L}, d) \longrightarrow \tilde{\pi}^{*} \overline{\mathfrak{M}}_{1, k}(\mathcal{L}, d) .
$$

All sections of $\tilde{\pi}^{*} \overline{\mathfrak{M}}_{1, k}(\mathcal{L}, d)$ must in fact be sections of $\widetilde{\mathfrak{M}}_{1, k}^{0}(\mathcal{L}, d)$ and thus the sheaf $\pi_{*} \tilde{\pi}^{*} \operatorname{ev}^{*} \mathcal{O}_{\mathbb{P}}(a)$ is indeed locally free. The bundle map

$$
\widetilde{\mathfrak{D}}_{\sigma ; B} \equiv \widetilde{\mathfrak{D}}_{\left([m], J_{B}\right)}
$$

of the second statement of Theorem 1.2 is described in Section 5.2. It is the "vertical" part of the natural extension of the bundle map $\widetilde{\mathcal{D}}_{\sigma ; B}$ from stable maps into $\mathbb{P}^{n}$ to stable maps into $\mathcal{L}$. Finally, the last statement of Theorem 1.2 is a consequence of the last statement of Theorem 1.1; see Lemma 5.2. At this point, this observation does not appear to have any applications though.

Remark By applying the methods of Section 5 and of [25], it should be possible to show that the standard scheme structure on $\overline{\mathfrak{M}}_{1, k}\left(\mathbb{P}^{n}, d\right)$ is in fact reduced.

\subsection{Outline of the main desingularization construction}

The main blowup construction of this paper is contained in Sections 4.2 and 4.3. It is a sequence of idealized blowups along smooth subvarieties. In other words, the blowup locus $\overline{\mathfrak{M}}_{1, \sigma}^{\sigma-1}$ at each step comes with an idealized normal bundle $\mathcal{N}^{\text {ide }}$. It is a vector bundle (of the smallest possible rank) containing the normal cone $\mathcal{N}$ for $\overline{\mathfrak{M}}_{1, \sigma}^{\sigma-1}$. After taking the usual blowup of the ambient space along $\overline{\mathfrak{M}}_{1, \sigma}^{\sigma-1}$, we attach the idealized exceptional divisor

$$
\mathcal{E}^{\text {ide }} \equiv \mathbb{P} \mathcal{N}^{\text {ide }}
$$

along the usual exceptional divisor

$$
\mathcal{E} \equiv \mathbb{P} \mathcal{N} \subset \mathcal{E}^{\text {ide }}
$$

The blowup construction summarized in Theorem 1.1 is contained in the idealized blowup construction of Section 4. The latter turns out to be more convenient for describing the proper transforms of $\overline{\mathfrak{M}}_{1, k}^{0}\left(\mathbb{P}^{n}, d\right)$, including at the final stage, ie $\widetilde{\mathfrak{M}}_{1, k}^{0}\left(\mathbb{P}^{n}, d\right)$.

The ambient space $\overline{\mathfrak{M}}_{1, k}^{\sigma}$ at each step $\sigma \in\{0\} \sqcup \mathcal{A}_{1}(d, k)$ of the blowup construction contains a subvariety $\overrightarrow{\mathfrak{M}}_{1, \sigma^{*}}^{\sigma}$ for each $\sigma^{*} \in \mathcal{A}_{1}(d, k)$. We take $\overline{\mathfrak{M}}_{1, \sigma}^{\sigma}$ to be the idealized 
exceptional divisor for the idealized blowup just constructed, ie along $\overline{\mathfrak{M}}_{1, \sigma}^{\sigma-1}$. If $\sigma^{*}<\sigma$ or $\sigma^{*}>\sigma, \overline{\mathfrak{M}}_{1, \sigma^{*}}^{\sigma}$ is the proper transform of $\overline{\mathfrak{M}}_{1, \sigma^{*}}^{\sigma^{*}}$ or $\overline{\mathfrak{M}}_{1, \sigma^{*}}\left(\mathbb{P}^{n}, d\right)$, respectively.

Every immersion $\iota_{\sigma^{*}}$ of Section 1.2 comes with an idealized normal bundle $\mathcal{N}_{\iota_{\sigma^{*}}}^{\text {ide }}$. It is a vector bundle of the smallest possible rank containing the normal cone to the immersion $\iota_{\sigma}$ (see Definition 4.1). It is given by

$$
\mathcal{N}_{l_{\sigma^{*}}}^{\text {ide }}=\bigoplus_{i \in\left[m^{*}\right]} \pi_{P}^{*} L_{i} \otimes \pi_{B}^{*} \pi_{i}^{*} L_{0} \quad \text { if } \quad \sigma^{*}=\left(m^{*} ; J_{P}^{*}, J_{B}^{*}\right)
$$

where $\quad \pi_{P}, \pi_{B}: \overline{\mathcal{M}}_{\sigma^{*} ; P} \times \overline{\mathfrak{M}}_{\sigma^{*} ; B}\left(\mathbb{P}^{n}, d\right) \longrightarrow \overline{\mathcal{M}}_{\sigma^{*} ; P}, \overline{\mathfrak{M}}_{\sigma^{*} ; B}\left(\mathbb{P}^{n}, d\right)$

are the component projection maps. In the case of Figure $1, \mathcal{N}_{l_{\sigma^{*}}}^{\text {ide }}$ is a rank-three vector bundle encoding the potential smoothings of the three nodes. At each step $\sigma$ of the blowup construction, $\iota_{\sigma^{*}}$ induces an immersion $\iota_{\sigma, \sigma^{*}}$ onto $\overline{\mathfrak{M}}_{1, \sigma^{*}}^{\sigma}$. Like the domain of $\iota_{\sigma^{*}}$, the domain of $\iota_{\sigma, \sigma^{*}}$ splits as a Cartesian product. If $\sigma^{*}>\sigma$, the second component of the domain does not change from the previous step, while the first is modified by blowing up along a collection of disjoint subvarieties, as specified by the inductive assumption (I9) in Section 4.3. The idealized normal bundle $\mathcal{N}_{\iota_{\sigma, \sigma^{*}}}^{\text {ide }}$ is obtained from $\mathcal{N}_{l_{\sigma-1, \sigma^{*}}}^{\text {ide }}$ by twisting the first factor in each summand by a subset of the exceptional divisors, as specified by the inductive assumption (I11). These blowup and twisting procedures correspond to several interchangeable steps in the blowup construction of Section 2.3. For $\sigma^{*}=\sigma$, the first component in the domain of $\iota_{\sigma-1, \sigma}$ has already been blown up all the way to $\widetilde{\mathcal{M}}_{\sigma ; P}$ and the first component of every summand of $\mathcal{N}_{l_{\sigma-1, \sigma}}^{\text {ide }}$ has already twisted to the universal tangent line bundle $\mathbb{L}$, ie

$$
\mathcal{N}_{l_{\sigma-1, \sigma}}^{\text {ide }}=\bigoplus_{i \in[m]} \pi_{P}^{*} \mathbb{L} \otimes \pi_{B}^{*} \pi_{i}^{*} L_{0}=\pi_{P}^{*} \mathbb{L} \otimes \pi_{B}^{*} F_{\sigma ; B} \longrightarrow \widetilde{\mathcal{M}}_{\sigma ; P} \times \overline{\mathfrak{M}}_{\sigma ; B}\left(\mathbb{P}^{n}, d\right),
$$

if $\sigma=\left(m ; J_{P}, J_{B}\right)$. In particular, the domain of $\iota_{\sigma, \sigma}$,

$$
\mathbb{P}_{\mathcal{N}^{\text {ide }}}^{\text {ide }}=\widetilde{\mathcal{M}}_{\sigma ; P} \times \mathbb{P} F_{\sigma ; B},
$$

still splits as a Cartesian product! The idealized normal bundle for $\iota_{\sigma, \sigma}$ is the tautological line for $\mathbb{P} \mathcal{N}_{l_{\sigma-1, \sigma}}^{\text {ide }}$ :

$$
\mathcal{N}_{l_{\sigma, \sigma}}^{\text {ide }}=\gamma_{\mathcal{N}_{l_{\sigma-1, \sigma}}^{\text {ide }}}=\pi_{P}^{*} \mathbb{L} \otimes \pi_{B}^{*} \gamma_{F_{\sigma ; B}} \equiv \pi_{P}^{*} \mathbb{L} \otimes \pi_{B}^{*} \gamma_{\sigma ; B}
$$

On the other hand, if $\sigma^{*}<\sigma$, the domain of $\iota_{\sigma, \sigma^{*}}$ is obtained from the domain of $\iota_{\sigma-1, \sigma^{*}}$ by blowing up the second component along a collection of disjoint subvarieties, as specified by the inductive assumption (I4) in Section 4.3. This corresponds to several interchangeable steps of the blowup construction in Section 3.4. By the time 
we are done with the last step of the blowup construction in Section 4.3, $\mathbb{P} F_{\sigma ; B}$ has been blown up all the way to $\widetilde{\mathfrak{M}}_{\sigma ; B}\left(\mathbb{P}^{n}, d\right)$. In the $\sigma^{*}<\sigma$ case,

$$
\mathcal{N}_{\iota_{\sigma, \sigma^{*}}}^{\text {ide }}=\mathcal{N}_{\iota_{\sigma-1, \sigma^{*}}^{\text {ide }}},
$$

since $\overline{\mathfrak{M}}_{1, \sigma^{*}}^{\sigma-1}$ is transverse to $\overline{\mathfrak{M}}_{1, \sigma}^{\sigma-1}$.

We study the proper transform $\overline{\mathfrak{M}}_{1,(0)}^{\sigma}$ of $\overline{\mathfrak{M}}_{1, k}^{0}\left(\mathbb{P}^{n}, d\right)$ in $\overline{\mathfrak{M}}_{1, k}^{\sigma}$ by looking at the structure of

$$
\overline{\mathcal{Z}}_{\sigma^{*}}^{\sigma}=\iota_{\sigma, \sigma^{*}}^{-1}\left(\overline{\mathfrak{M}}_{1,(0)}^{\sigma}\right) .
$$

Given a finite set $J$, there are natural bundle sections

$$
s_{j} \in \Gamma\left(\overline{\mathcal{M}}_{1, J} ; L_{j}^{*} \otimes \mathbb{E}^{*}\right), j \in J, \quad \text { and } \quad \mathcal{D}_{0} \in \Gamma\left(\overline{\mathfrak{M}}_{0,\{0\} \sqcup J}\left(\mathbb{P}^{n}, d\right) ; L_{0}^{*} \otimes \mathrm{ev}_{0}^{*} T \mathbb{P}^{n}\right) ;
$$

see Sections 2.2 and 3.2, respectively. By Lemma 4.10, the intersection of

$$
\overline{\mathcal{Z}}_{\sigma^{*}}^{0} \equiv \iota_{\sigma^{*}}^{-1}\left(\overline{\mathfrak{M}}_{1, k}^{0}\left(\mathbb{P}^{n}, d\right)\right)
$$

with the main stratum $\mathcal{M}_{\sigma^{*} ; P} \times \mathfrak{M}_{\sigma^{*} ; B}\left(\mathbb{P}^{n}, d\right)$ of $\overline{\mathcal{M}}_{\sigma^{*} ; P} \times \overline{\mathfrak{M}}_{\sigma^{*} ; B}\left(\mathbb{P}^{n}, d\right)$ is

$$
\mathcal{Z}_{\sigma^{*}}^{0}=\left\{b \in \mathcal{M}_{\sigma^{*} ; P} \times \mathfrak{M}_{\sigma^{*} ; B}\left(\mathbb{P}^{n}, d\right):\left.\operatorname{ker} \mathcal{D}_{\sigma^{*}}\right|_{b} \neq\{0\}\right\}
$$

where $\quad \mathcal{D}_{\sigma^{*}} \in \Gamma\left(\overline{\mathcal{M}}_{\sigma^{*} ; P} \times \overline{\mathfrak{M}}_{\sigma^{*} ; B}\left(\mathbb{P}^{n}, d\right) ; \operatorname{Hom}\left(\mathcal{N}_{l_{\sigma^{*}}}^{\text {ide }}, \pi_{P}^{*} \mathbb{E}^{*} \otimes \pi_{B}^{*} \operatorname{ev}_{0}^{*} T \mathbb{P}^{n}\right)\right)$,

$$
\left.\mathcal{D}_{\sigma^{*}}\right|_{\pi_{P}^{*} L_{i} \otimes \pi_{B}^{*} \pi_{i}^{*} L_{0}}=\pi_{P}^{*} s_{i} \otimes \pi_{B}^{*} \pi_{i}^{*} \mathcal{D}_{0}, \quad \forall i \in\left[m^{*}\right] .
$$

In addition, if $\mathcal{N} \overline{\mathcal{Z}}_{\sigma^{*}}^{\sigma} \subset \mathcal{N}_{\iota_{\sigma, \sigma^{*}}}^{\text {ide }}$ is the normal cone for the immersion $\left.\iota_{\sigma, \sigma^{*}}\right|_{\overline{\mathcal{Z}}_{\sigma^{*}}^{\sigma}}$ into $\overline{\mathfrak{M}}_{1,(0)}^{\sigma}$, then

$$
\left.\mathcal{N} \overline{\mathcal{Z}}_{\sigma^{*}}^{0}\right|_{\mathcal{Z}_{\sigma^{*}}^{0}}=\left.\operatorname{ker} \mathcal{D}_{\sigma^{*}}\right|_{\mathcal{Z}_{\sigma^{*}}^{0}}
$$

and $\mathcal{N} \overline{\mathcal{Z}}_{\sigma^{*}}^{0}$ is the closure of $\left.\mathcal{N} \overline{\mathcal{Z}}_{\sigma^{*}}^{0}\right|_{\mathcal{Z}_{\sigma^{*}}^{0}}$ in $\mathcal{N}_{l_{\sigma^{*}}}^{\text {ide }}$. By Lemma $4.5, \mathcal{N} \overline{\mathcal{Z}}_{\sigma^{*}}^{\sigma}$ is still the closure of $\left.\mathcal{N} \overline{\mathcal{Z}}_{\sigma^{*}}^{0}\right|_{\mathcal{Z}_{\sigma^{*}}^{0}}$, but now in $\mathcal{N}_{l_{\sigma, \sigma^{*}}}^{\text {ide }}$, for all $\sigma<\sigma^{*}$. In Section 2.3, we construct a nonvanishing section

$$
\tilde{s}_{i} \in \Gamma\left(\overline{\mathcal{M}}_{1, J} ; \mathbb{L}^{*} \otimes \mathbb{E}^{*}\right) \approx \Gamma\left(\overline{\mathcal{M}}_{1, J} ; \mathbb{C}\right)
$$

obtained by twisting $s_{i}$ by some exceptional divisors. Since $\tilde{s}_{i}$ agrees with $s_{i}$ on $\mathcal{M}_{\sigma^{*} ; P}$, we can replace $s_{i}$ with $\tilde{s}_{i}$ in the descriptions of $\mathcal{D}_{\sigma^{*}}, \mathcal{Z}_{\sigma^{*}}^{0}$, and $\left.\mathcal{N} \overline{\mathcal{Z}}_{\sigma^{*}}^{0}\right|_{\mathcal{Z}_{\sigma^{*}}^{0}}$ 
above. In particular, $\mathcal{N} \overline{\mathcal{Z}}_{\sigma^{*}}^{\sigma^{*}-1}$ is the closure of

$$
\left.\mathcal{N} \overline{\mathcal{Z}}_{\sigma^{*}}^{0}\right|_{\mathcal{Z}_{\sigma^{*}}^{0}}=\left.\pi_{P}^{*} \mathbb{L} \otimes \pi_{B}^{*} \operatorname{ker} \mathcal{D}_{\sigma^{*} ; B}\right|_{\mathcal{Z}_{\sigma^{*}}^{0}} \subset \pi_{P}^{*} \mathbb{L} \otimes \pi_{B}^{*} F_{\sigma^{*} ; B}
$$

where

$$
\begin{aligned}
\mathcal{D}_{\sigma^{*} ; B} \in \Gamma\left(\overline{\mathfrak{M}}_{\sigma^{*} ; B}\left(\mathbb{P}^{n}, d\right) ; \operatorname{Hom}\left(F_{\sigma^{*} ; B}, \mathrm{ev}_{0}^{*} T \mathbb{P}^{n}\right)\right), \\
\left.\mathcal{D}_{\sigma^{*} ; B}\right|_{\pi_{i}^{*} L_{0}}=\pi_{i}^{*} \mathcal{D}_{0} \forall i \in\left[m^{*}\right] .
\end{aligned}
$$

The bundle homomorphism $\mathcal{D}_{\sigma^{*} ; B}$ induces a section

$$
\widetilde{\mathcal{D}}_{0} \in \Gamma\left(\mathbb{P} F_{\sigma^{*} ; B} ; \gamma_{\sigma ; B}^{*} \otimes \pi_{\mathbb{P} F_{\sigma^{*} ; B}}^{*} \mathrm{ev}_{0}^{*} T \mathbb{P}^{n}\right) .
$$

By the previous paragraph and Lemma $4.5, \overline{\mathcal{Z}}_{\sigma^{*}}^{\sigma^{*}}$ is the closure of

$$
\widetilde{\mathcal{M}}_{\sigma^{*} ; P} \times\left.\widetilde{\mathcal{D}}_{0}^{-1}(0) \cap \mathbb{P} F_{\sigma^{*} ; B}\right|_{\mathfrak{M}_{\sigma^{*} ; B}\left(\mathbb{P}^{n}, d\right)} \subset \widetilde{\mathcal{M}}_{\sigma^{*} ; P} \times \mathbb{P} F_{\sigma^{*} ; B}
$$

and

$$
\mathcal{N} \overline{\mathcal{Z}}_{\sigma^{*}}^{\sigma^{*}}=\left.\pi_{P}^{*} \mathbb{L} \otimes \pi_{B}^{*} \gamma_{\sigma ; B}\right|_{\overline{\mathcal{Z}}_{\sigma^{*}}^{\sigma^{*}}}
$$

Since $\widetilde{\mathfrak{M}}_{1, k}^{0}\left(\mathbb{P}^{n}, d\right) \equiv \widetilde{\mathfrak{M}}_{1, k}^{\sigma_{\max }}\left(\mathbb{P}^{n}, d\right)$ is the proper transform of $\overline{\mathfrak{M}}_{1,(0)}^{\sigma^{*}}$ in $\overline{\mathfrak{M}}_{1, k}^{\sigma_{\max }}$,

$$
\widetilde{\mathcal{Z}}_{\sigma^{*}} \equiv \iota_{\sigma_{\max }, \sigma^{*}}^{-1}\left(\widetilde{\mathfrak{M}}_{1, k}^{0}\left(\mathbb{P}^{n}, d\right)\right)
$$

is still the closure of

$$
\widetilde{\mathcal{M}}_{\sigma^{*} ; P} \times\left.\widetilde{\mathcal{D}}_{0}^{-1}(0) \cap \mathbb{P} F_{\sigma^{*} ; B}\right|_{\mathfrak{M}_{\sigma^{*} ; B}\left(\mathbb{P}^{n}, d\right)} \subset \widetilde{\mathcal{M}}_{\sigma^{*} ; P} \times \widetilde{\mathfrak{M}}_{\sigma^{*} ; B}\left(\mathbb{P}^{n}, d\right) .
$$

On the other hand, in the process of constructing the blowup $\widetilde{\mathfrak{M}}_{\sigma ; B}\left(\mathbb{P}^{n}, d\right)$ of $\mathbb{P} F_{\sigma^{*} ; B}$ in Section 3.4, we also define a bundle section

$$
\widetilde{\mathcal{D}}_{\sigma^{*} ; B} \in \Gamma\left(\widetilde{\mathfrak{M}}_{\sigma^{*} ; B}\left(\mathbb{P}^{n}, d\right) ; \mathbb{E}_{\sigma ; B}^{*} \otimes \tilde{\pi}_{\sigma ; B}^{*} \pi_{\mathbb{P} F_{\sigma^{*} ; B}}^{*} \mathrm{ev}_{0}^{*} T \mathbb{P}^{n}\right)
$$

by twisting $\widetilde{\mathcal{D}}_{0}$ by the exceptional divisors. In particular,

$$
\left.\widetilde{\mathcal{D}}_{\sigma^{*} ; B}^{-1}(0) \cap \mathbb{P} F_{\sigma^{*} ; B}\right|_{\mathfrak{M}_{\sigma^{*} ; B}\left(\mathbb{P}^{n}, d\right)}=\left.\widetilde{\mathcal{D}}_{0}^{-1}(0) \cap \mathbb{P} F_{\sigma^{*} ; B}\right|_{\mathfrak{M}_{\sigma^{*} ; B}\left(\mathbb{P}^{n}, d\right)} .
$$

Since $\widetilde{\mathcal{D}}_{\sigma^{*} ; B}$ is transverse to the zero set, we conclude that

$$
\widetilde{\mathcal{Z}}_{\sigma^{*}}=\widetilde{\mathcal{M}}_{\sigma^{*} ; P} \times \widetilde{\mathcal{D}}_{\sigma^{*} ; B}^{-1}(0),
$$

as stated in Section 1.2.

Finally, the role played by the blowup construction of Section 2.4 in the blowup construction of Section 3 is similar to the role played by the construction of Section 2.3 in the construction of Section 4. In the case of Section 3, we blow up a moduli space of genus-zero stable maps, $\mathbb{P} F_{(\aleph, J)}$, along certain subvarieties $\widetilde{\mathfrak{M}}_{0, \varrho}^{0}$ and their proper transforms. These subvarieties are images of natural node-identifying immersions $\iota_{0, \varrho}$. 
The domain of $\iota_{0, \varrho}$ splits as the Cartesian product of a moduli space of genus-zero curves and a moduli space of genus-zero maps, defined in Sections 2.4 and 3.3, respectively. As we modify $\widetilde{\mathfrak{M}}_{0, \varrho}^{0}$ by taking its proper transforms in the blowups of $\mathbb{P} F_{(\aleph, J)}$ constructed in Section 3.4, the first factor in the domain of the corresponding immersion changes by blowups along collections of smooth disjoint subvarieties, as specified by the inductive assumption (I6). This change corresponds to several interchangeable steps in the blowup construction of Section 2.4. By the time we are ready to blow up the proper transform of $\widetilde{\mathfrak{M}}_{0, \varrho}^{0}$, the first component of the domain of the corresponding immersion has been blown up all the way to $\widetilde{\mathcal{M}}_{0, \rho_{P}}$, the end result in the blowup construction of Section 2.4.

In the blowup construction of Section 3.4, we twist a natural bundle section

$$
\widetilde{\mathcal{D}}_{0} \in \Gamma\left(\mathbb{P} F_{(\aleph, J)} ; \gamma_{(\aleph, J)}^{*} \otimes \pi_{\mathbb{P} F_{(\aleph, J)}}^{*} \operatorname{ev}_{0}^{*} T \mathbb{P}^{n}\right)
$$

by the exceptional divisors to a bundle section

$$
\widetilde{\mathcal{D}}_{(\aleph, J)} \in \Gamma\left(\widetilde{\mathfrak{M}}_{(\aleph, J)}\left(\mathbb{P}^{n}, d\right) ; \widetilde{\mathbb{E}}^{*} \otimes \pi_{0,(\aleph, J)}^{*} \pi_{\mathbb{P} F_{(\aleph, J)}}^{*} \mathrm{ev}_{0}^{*} T \mathbb{P}^{n}\right) .
$$

The two sections enter in an essential way in the main blowup construction of this paper. It is also essential that $\widetilde{\mathcal{D}}_{(\aleph, J)}$ is transverse to the zero set. The section $\widetilde{\mathcal{D}}_{0}$ is transverse to the zero set outside of the subvarieties $\widetilde{\mathfrak{M}}_{0, \varrho}^{0}$ and vanishes identically along $\widetilde{\mathfrak{M}}_{0, \varrho}^{0}$. Its derivative in the normal direction to $\iota_{0, \varrho}$ is described by Lemma 3.11, using Lemma 3.8. The bundle sections $s_{i}$ over a moduli space of genus-zero curves defined in Section 2.2 and modified in Section 2.4 enter into the expression of Lemma 3.8. In fact, this expression is identical to the expression for $\mathcal{D}_{\sigma^{*}}$ above, ie in the genus-one case. We use Lemma 3.11 to show that with each newly twisted version of $\widetilde{\mathcal{D}}_{0}$ is transverse to the zero set outside of the proper transforms of the remaining subvarieties $\overline{\mathfrak{M}}_{0, \varrho}^{0}$, ie the ones that have not been blown up yet; see the inductive assumption (I4) in Section 3.4. In particular, at the end of the blowup construction of Section 3.4, we end up with a twisted version of $\widetilde{\mathcal{D}}_{0}$, which we call $\widetilde{\mathcal{D}}_{(\aleph, J)}$, which is transverse to the zero set.

\subsection{Localization data}

Suppose the group $G=\left(S^{1}\right)^{n+1}$ or $G=\left(\mathbb{C}^{*}\right)^{n+1}$ acts in a natural way on the projective space $\mathbb{P}^{n}$. In particular, the fixed locus consists of $n+1$ points, which we denote by $p_{0}, \ldots, p_{n}$, and the only curves preserved by $G$ are the lines passing through pairs of fixed points. The $G$-action on $\mathbb{P}^{n}$ lifts to an action on $\overline{\mathfrak{M}}_{1, k}\left(\mathbb{P}^{n}, d\right)$ and on $\widetilde{\mathfrak{M}}_{1, k}^{0}\left(\mathbb{P}^{n}, d\right)$. The fixed loci of these two actions that are contained in $\mathfrak{M}_{1, k}^{\text {eff }}\left(\mathbb{P}^{n}, d\right)$ and their normal bundles are the same and are described in Sections 27.3 and 27.4 of Mirror Symmetry [10]. We note that the four-term exact sequence [10, (27.6)] applies to such loci. 
In this subsection, we describe the fixed loci of the $G$-action on $\widetilde{\mathfrak{M}}_{1, k}^{0}\left(\mathbb{P}^{n}, d\right)$ that are contained in $\partial \widetilde{\mathfrak{M}}_{1, k}^{0}\left(\mathbb{P}^{n}, d\right)$ and their normal bundles. To simplify the discussion, we ignore all automorphism groups until the very end of this subsection.

The boundary fixed loci $\widetilde{\mathcal{Z}}_{\widetilde{\Gamma}}$ will be indexed by refined decorated rooted trees $\widetilde{\Gamma}$. Figure 5 shows such a tree $\widetilde{\Gamma}$ and the corresponding decorated graph $\Gamma=\pi(\widetilde{\Gamma})$. In $\left[10\right.$, Section 27.3] the fixed loci $\mathcal{Z}_{\Gamma}$ of the $G$-action on $\overline{\mathfrak{M}}_{g, k}\left(\mathbb{P}^{n}, d\right)$ are indexed by decorated graphs $\Gamma$. If $\Gamma$ is a decorated graph such that $\mathcal{Z}_{\Gamma}$ is a $G$-fixed locus contained in $\partial \overline{\mathfrak{M}}_{1, k}\left(\mathbb{P}^{n}, d\right)$, we will have

$$
\mathcal{Z}_{\Gamma} \cap \overline{\mathfrak{M}}_{1, k}^{0}\left(\mathbb{P}^{n}, d\right)=\tilde{\pi}\left(\underset{\pi(\widetilde{\Gamma})=\Gamma}{\bigsqcup_{\mathcal{Z}_{\tilde{\Gamma}}}}\right),
$$

where $\widetilde{\Gamma}$ denotes a refined decorated rooted tree.

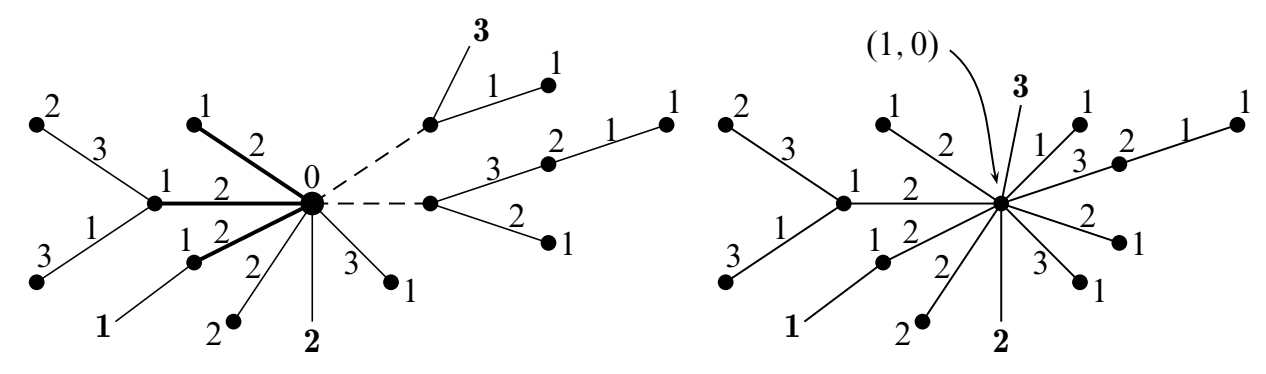

Figure 5: A refined decorated rooted tree and its decorated graph

We now formally describe what we mean by a refined decorated rooted tree and its corresponding decorated graph. A graph consists of a set Ver of vertices and a collection Edg of edges, ie of two-element subsets of Ver. In Figure 5, the vertices are represented by dots, while each edge $\left\{v_{1}, v_{2}\right\}$ is shown as the line segment between $v_{1}$ and $v_{2}$. A graph is a tree if it contains no loops, ie the set Edg contains no subset of the form

$$
\left\{\left\{v_{1}, v_{2}\right\},\left\{v_{2}, v_{3}\right\}, \ldots,\left\{v_{N}, v_{1}\right\}\right\}, \quad v_{1}, \ldots, v_{N} \in \text { Ver, } N \geq 1 .
$$

A tree is rooted if Ver contains a distinguished element $v_{0}$. It is represented by the large dot in the first diagram of Figure 5. A rooted tree is refined if Ver- $\left\{v_{0}\right\}$ contains two, possibly empty, distinguished subsets $\mathrm{Ver}_{+}$and $\mathrm{Ver}_{0}$ such that

$$
\operatorname{Ver}_{+} \cap \operatorname{Ver}_{0}=\varnothing \quad \text { and } \quad\left\{v_{0}, v\right\} \in \operatorname{Edg} \forall v \in \operatorname{Ver}_{+} \cup \operatorname{Ver}_{0} .
$$

We put

$$
\operatorname{Edg}_{+}=\left\{\left\{v_{0}, v\right\}: v \in \operatorname{Ver}_{+}\right\} \text {and } \operatorname{Edg}_{0}=\left\{\left\{v_{0}, v\right\}: v \in \operatorname{Ver}_{0}\right\}
$$


The elements of Edg $g_{+}$and $\operatorname{Edg}_{0}$ are shown in the first diagram of Figure 5 as the thick solid lines and the thin dashed lines, respectively. Finally, a refined decorated rooted tree is a tuple

$$
\widetilde{\Gamma}=\left(\text { Ver, Edg; } v_{0} ; \operatorname{Ver}_{+}, \operatorname{Ver}_{0} ; \mu, \mathfrak{d}, \eta\right),
$$

where (Ver, Edg; $v_{0} ; \operatorname{Ver}_{+}, \operatorname{Ver}_{0}$ ) is refined rooted tree and

$$
\mu: \text { Ver- }- \text { Ver }_{0} \longrightarrow\{0, \ldots, n\}, \quad \mathfrak{d}: \operatorname{Edg}-\operatorname{Edg}_{0} \longrightarrow \mathbb{Z}^{+}, \quad \eta:\{1, \ldots, k\} \longrightarrow \text { Ver }
$$

are maps such that

(i) $\mu\left(v_{1}\right)=\mu\left(v_{2}\right)$ and $\mathfrak{d}\left(\left\{v_{0}, v_{1}\right\}\right)=\mathfrak{d}\left(\left\{v_{0}, v_{2}\right\}\right)$ for all $v_{1}, v_{2} \in \operatorname{Ver}_{+}$;

(ii) if $v_{1} \in \mathrm{Ver}_{+}, v_{2} \in \mathrm{Ver}-\mathrm{Ver}_{0}-\mathrm{Ver}_{+}$, and $\left\{v_{0}, v_{2}\right\} \in \mathrm{Edg}$, then

$$
\mu\left(v_{1}\right) \neq \mu\left(v_{2}\right) \quad \text { or } \quad \mathfrak{d}\left(\left\{v_{0}, v_{1}\right\}\right) \neq \mathfrak{d}\left(\left\{v_{0}, v_{2}\right\}\right) \text {; }
$$

(iii) if $\left\{v_{1}, v_{2}\right\} \in \operatorname{Edg}$ and $v_{2} \notin \operatorname{Ver}_{0} \cup\left\{v_{0}\right\}$, then

$$
\mu\left(v_{2}\right) \neq \mu\left(v_{1}\right) \quad \text { if } v_{1} \notin \operatorname{Ver}_{0} \quad \text { and } \quad \mu\left(v_{2}\right) \neq \mu\left(v_{0}\right) \quad \text { if } v_{1} \in \operatorname{Ver}_{0} ;
$$

(iv) if $v_{1} \in \operatorname{Ver}_{0}$, then $\left\{v_{1}, v_{2}\right\} \in \operatorname{Edg}$ for some $v_{2} \in \operatorname{Ver}-\left\{v_{0}\right\}$ and

$$
\operatorname{val}\left(v_{1}\right) \equiv\left|\left\{v_{2} \in \operatorname{Ver}:\left\{v_{1}, v_{2}\right\} \in \operatorname{Edg}\right\}\right|+\mid\left\{l \in[k]: \eta(l)=v_{1} \mid \geq 3 ;\right.
$$

(v) $\sum_{e \in \operatorname{Edg}_{+}} \mathfrak{d}(e) \geq 2$.

In Figure 5, the value of the map $\mu$ on each vertex, not in $\operatorname{Ver}_{0}$, is indicated by the number next to the vertex. Similarly, the value of the map $\mathfrak{d}$ on each edge, not in $\operatorname{Edg}_{0}$, is indicated by the number next to the edge. The elements of the set $[k]=[3]$ are shown in bold face. Each of them is linked by a line segment to its image under $\eta$. The first condition above implies that all of the thick edges have the same labels, and so do their vertices, other than the root $v_{0}$. By the second condition, the set of thick edges is a maximal set of edges leaving $v_{0}$ which satisfies the first condition. By the third condition, no two consecutive vertex labels are the same. By the fourth condition, there are at least two solid lines, at least one of which is an edge, leaving from every vertex which is connected to the root by a dashed line. The final condition implies that either the set Edg $g_{+}$contains at least two elements or its only element is marked by at least 2 .

A decorated graph is a tuple

$$
\Gamma=(\text { Ver, } \operatorname{Edg} ; g, \mu, \mathfrak{d}, \eta),
$$

where (Ver, Edg) is a graph and

$$
g: \text { Ver } \longrightarrow \overline{\mathbb{Z}}^{+}, \quad \mu: \text { Ver } \longrightarrow\{0, \ldots, n\}, \quad \mathfrak{d}: \operatorname{Edg} \longrightarrow \mathbb{Z}^{+}, \quad \eta:[k] \longrightarrow \text { Ver }
$$


are maps such that

$$
\mu\left(v_{1}\right) \neq \mu\left(v_{2}\right) \quad \text { if } \quad\left\{v_{1}, v_{2}\right\} \in \mathrm{Edg} .
$$

The domain $[k]$ of the map $\eta$ can be replaced by any finite set. A decorated graph can be represented graphically as in the second diagram of Figure 5. In this case, every vertex $v$ should be labeled by the pair $(g(v), \mu(v))$. However, we drop the first entry if it is zero. If $\widetilde{\Gamma}$ is a refined decorated rooted tree as in (1-8), the corresponding decorated graph $\Gamma$ is obtained by identifying all elements of $\operatorname{Ver}_{0}$ with $v_{0}$, dropping $\mathrm{Edg}_{0}$ from Edg, and setting

$$
g(v)= \begin{cases}1, & \text { if } v=v_{0} \\ 0, & \text { otherwise }\end{cases}
$$

In terms of the first diagram in Figure 5, this procedure corresponds to contracting the dashed edges and adding 1 to the label for $v_{0}$.

The fixed locus $\mathcal{Z}_{\Gamma}$ of $\overline{\mathfrak{M}}_{1, k}\left(\mathbb{P}^{n}, d\right)$ consists of the stable maps $u$ from a genus-one nodal curve $\Sigma_{u}$ with $k$ marked points into $\mathbb{P}^{n}$ that satisfy the following conditions. The components of $\Sigma_{u}$ on which the map $u$ is not constant are rational and correspond to the edges of $\Gamma$. Furthermore, if $e=\left\{v_{1}, v_{2}\right\}$ is an edge, the restriction of $u$ to the component $\Sigma_{u, e}$ corresponding to $e$ is a degree- $\mathfrak{d}(e)$ cover of the line

$$
\mathbb{P}_{p_{\mu\left(v_{1}\right)}, p_{\mu\left(v_{2}\right)}}^{1} \subset \mathbb{P}^{n}
$$

passing through the fixed points $p_{\mu\left(v_{1}\right)}$ and $p_{\mu\left(v_{2}\right)}$. The map $\left.u\right|_{\Sigma_{u, e}}$ is ramified only over $p_{\mu\left(v_{1}\right)}$ and $p_{\mu\left(v_{2}\right)}$. In particular, $\left.u\right|_{\Sigma_{u, e}}$ is unique up to isomorphism. The remaining, contracted, components of $\Sigma_{u}$ correspond to the vertices $v \in$ Ver such that

$$
\operatorname{val}(v)+g(v) \geq 3
$$

For such a vertex $v, g(v)$ specifies the genus of the component corresponding to $v$. The map $u$ takes this component to the fixed point $\mu(v)$. Thus,

$$
\mathcal{Z}_{\Gamma} \approx \overline{\mathcal{M}}_{\Gamma} \equiv \prod_{v \in \operatorname{Ver}} \overline{\mathcal{M}}_{g(v), \operatorname{val}(v)}
$$

[10, Section 27.3]. For the purposes of this definition, $\overline{\mathcal{M}}_{0,1}$ and $\overline{\mathcal{M}}_{0,2}$ denote onepoint spaces. For example, in the case of the second diagram in Figure 5,

$$
\mathcal{Z}_{\Gamma} \approx \overline{\mathcal{M}}_{\Gamma} \equiv \overline{\mathcal{M}}_{1,10} \times \overline{\mathcal{M}}_{0,3} \times \overline{\mathcal{M}}_{0,2}^{2} \times \overline{\mathcal{M}}_{0,1}^{8} \approx \overline{\mathcal{M}}_{1,10}
$$

In this case, $\mathcal{Z}_{\Gamma}$ is a locus in $\overline{\mathfrak{M}}_{1,3}\left(\mathbb{P}^{n}, 22\right)$, with $n \geq 3$. 
If $\widetilde{\Gamma}$ is a refined decorated rooted tree as in (1-8), we put

$$
\operatorname{Edg}\left(v_{0}\right)=\left\{\left\{v_{0}, v_{1}\right\} \in \operatorname{Edg}: v_{1} \in \operatorname{Ver}\right\} \text { and } J_{v_{0}}=\left\{l \in[k]: \mu(l)=v_{0}\right\} .
$$

Similarly, for each $v \in \operatorname{Ver}_{0}$, we set

$$
\operatorname{Edg}(v)=\left\{\left\{v, v_{1}\right\} \in \operatorname{Edg}: v_{1} \in \operatorname{Ver}-\left\{v_{0}\right\}\right\} \text { and } J_{v}=\{l \in[k]: \mu(l)=v\} .
$$

If $e=\left\{v, v_{1}\right\}$ is an element of $\operatorname{Edg}(v)$ for some $v \in \operatorname{Ver}_{0}$ or of $\operatorname{Edg}\left(v_{0}\right)-\operatorname{Edg}_{0}$ with $v=v_{0}$, let $\left(\operatorname{Ver}_{e}, \operatorname{Edg}_{e}\right)$ be the branch of the tree (Ver, Edg) beginning at $v$ with the edge $e$. We put

$$
J_{e}=\left\{l \in[k]: \mu(l) \in \operatorname{Ver}_{e}-\{v\}\right\} \quad \text { and } \quad d_{e}=\sum_{e^{\prime} \in \operatorname{Edg}_{e}} \mathfrak{d}\left(e^{\prime}\right) .
$$

Let $\widetilde{\Gamma}_{e}$ be the decorated graph defined by

$$
\widetilde{\Gamma}_{e}=\left(\operatorname{Ver}_{e}, \operatorname{Edg}_{e} ; g_{e} \equiv 0, \mu_{e},\left.\mathfrak{d}_{e} \equiv \mathfrak{d}\right|_{\operatorname{Edg}_{e}}, \eta_{e}\right) \text {, }
$$

where

$$
\mu_{e}\left(v^{\prime}\right)=\left\{\begin{array}{ll}
\mu\left(v^{\prime}\right), & \text { if } v^{\prime} \neq v ; \\
\mu\left(v_{0}\right), & \text { if } v^{\prime}=v ;
\end{array} \quad \eta_{e}:\{0\} \sqcup J_{e} \longrightarrow \operatorname{Ver}_{e}, \eta_{e}(l)= \begin{cases}\eta(l), & \text { if } l \in J_{e} ; \\
v, & \text { if } l=0 ;\end{cases}\right.
$$

see Figure 6 for two examples.

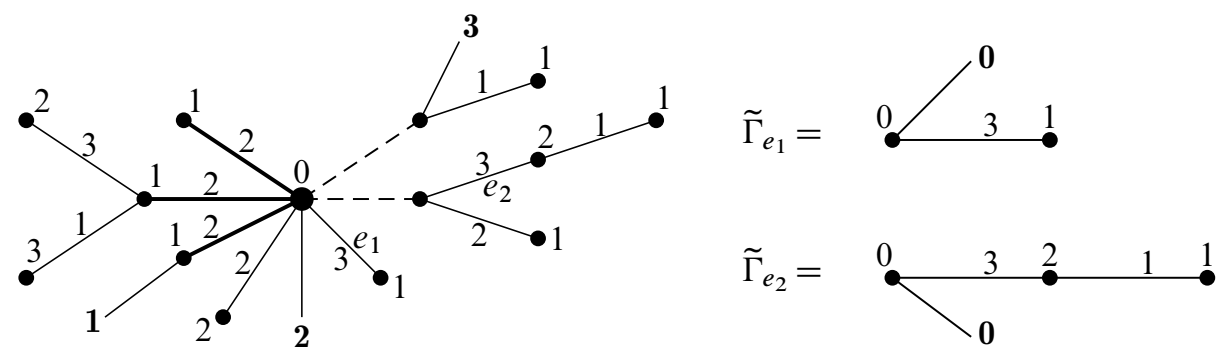

Figure 6: A refined decorated rooted tree and some of its components graphs

If $e$ is an element of $\operatorname{Edg}\left(v_{0}\right)-\operatorname{Edg}_{0}$ or of $\operatorname{Edg}(v)$ for some $v \in \operatorname{Ver}_{0}$, let

$$
\mathcal{Z}_{\widetilde{\Gamma}_{e}} \subset \overline{\mathfrak{M}}_{0,\{0\} \sqcup J_{e}}\left(\mathbb{P}^{n}, d_{e}\right)
$$


be the fixed locus corresponding to the decorated graph $\widetilde{\Gamma}_{e}$. We put

$$
\begin{gathered}
\sigma(\widetilde{\Gamma})=\left(\left|\operatorname{Edg}\left(v_{0}\right)\right| ; J_{v_{0}},[k]-J_{v_{0}}\right) \in \mathcal{A}_{1}(d, k), \quad \widetilde{\mathcal{M}}_{\widetilde{\Gamma} ; P}=\widetilde{\mathcal{M}}_{\sigma(\widetilde{\Gamma}) ; P} ; \\
\overline{\mathcal{Z}}_{\widetilde{\Gamma} ; B}=\prod_{e \in \operatorname{Edg}\left(v_{0}\right)-\operatorname{Edg}_{0}} \mathcal{Z}_{\widetilde{\Gamma}_{e}} \times \prod_{v \in \operatorname{Ver}_{0}}\left(\overline{\mathcal{M}}_{0,\{0\} \sqcup \operatorname{Edg}(v) \sqcup J_{v}} \times \prod_{e \in \operatorname{Edg}(v)} \mathcal{Z}_{\widetilde{\Gamma}_{e}}\right) \subset \overline{\mathfrak{M}}_{\sigma(\widetilde{\Gamma}) ; B}\left(\mathbb{P}^{n}, d\right) ; \\
F_{\widetilde{\Gamma} ; B}=\bigoplus_{e \in \operatorname{Edg}_{+}} L_{e ; 0} \subset F_{\sigma(\widetilde{\Gamma}) ; B} \longrightarrow \overline{\mathcal{Z}}_{\widetilde{\Gamma} ; B},
\end{gathered}
$$

where $L_{e ; 0} \longrightarrow \mathcal{Z}_{\widetilde{\Gamma}_{e}}$ is the tangent line bundle for the marked point 0 . If $e=\left\{v_{0}, v_{1}\right\}$ is an element of $\mathrm{Edg}_{+}$, let

$$
\mu_{+}(\widetilde{\Gamma})=\mu\left(v_{1}\right), \quad \mathfrak{d}_{+}(\widetilde{\Gamma})=\mathfrak{d}(e), \quad \operatorname{dim}_{+}(\widetilde{\Gamma})= \begin{cases}\left|\operatorname{Edg}_{+}\right|-2, & \text { if } \mathfrak{d}_{+}(\widetilde{\Gamma})=1 \\ \left|\operatorname{Edg}_{+}\right|-1, & \text { if } \mathfrak{d}_{+}(\widetilde{\Gamma}) \geq 2\end{cases}
$$

By the assumption (i) above, the numbers $\mu_{+}(\widetilde{\Gamma})$ and $\mathfrak{d}_{+}(\widetilde{\Gamma})$ are independent of the choice of $e \in \operatorname{Edg}_{+}$. Furthermore, if $e, e^{\prime} \in \operatorname{Edg}_{+}$, then the line bundles $L_{e ; 0}$ and $L_{e^{\prime} ; 0}$ are $G$-equivariantly isomorphic. Thus,

$$
F_{\widetilde{\Gamma} ; B} \approx \mathbb{C}^{\left|\mathrm{Edg}_{+}\right|} \otimes L_{e ; 0} \quad \text { if } \quad e \in \mathrm{Edg}_{+} .
$$

The group $G$ acts trivially on $\mathbb{C}^{\left|\mathrm{Edg}_{+}\right|}$. Let

$$
\begin{gathered}
F_{\widetilde{\Gamma} ; B}^{\prime}= \begin{cases}\left\{\left(w_{e}\right)_{e \in \mathrm{Edg}_{+}} \in \mathbb{C}^{\mathrm{Edg}}: \sum_{e \in \mathrm{Edg}_{+}} w_{e}=0\right\}, & \text { if } \mathfrak{d}_{+}(\widetilde{\Gamma})=1 ; \\
\mathbb{C}^{\mathrm{Edg}_{+}}, & \text {if } \mathfrak{d}_{+}(\tilde{\Gamma}) \geq 2 ;\end{cases} \\
\widetilde{\mathcal{Z}}_{\widetilde{\Gamma} ; B}=\mathbb{P}\left(F_{\widetilde{\Gamma} ; B}^{\prime} \otimes L_{e ; 0}\right) \approx \overline{\mathcal{Z}}_{\widetilde{\Gamma} ; B} \times \mathbb{P}^{\operatorname{dim}+(\widetilde{\Gamma})}
\end{gathered}
$$

While the moduli space $\widetilde{\mathfrak{M}}_{\sigma(\widetilde{\Gamma}) ; B}\left(\mathbb{P}^{n}, d\right)$ is a blowup of $\mathbb{P} F_{\sigma(\widetilde{\Gamma}) ; B}$, none of the blowup loci intersects $\widetilde{\mathcal{Z}}_{\widetilde{\Gamma} ; B}$. Thus,

$$
\widetilde{\mathcal{Z}}_{\widetilde{\Gamma} ; B} \subset \widetilde{\mathfrak{M}}_{\sigma(\widetilde{\Gamma}) ; B}\left(\mathbb{P}^{n}, d\right)
$$

In fact,

$$
\widetilde{\mathcal{Z}}_{\widetilde{\Gamma} ; B} \subset \widetilde{\mathcal{Z}}_{\sigma(\widetilde{\Gamma}) ; B}\left(\mathbb{P}^{n}, d\right)
$$

We put

$$
\widetilde{\mathcal{Z}}_{\widetilde{\Gamma}}=\widetilde{\mathcal{M}}_{\widetilde{\Gamma} ; P} \times \widetilde{\mathcal{Z}}_{\widetilde{\Gamma} ; B}
$$


By the above, $\widetilde{\mathcal{Z}}_{\widetilde{\Gamma}}$ is a fixed point locus in $\widetilde{\mathfrak{M}}_{1, k}^{0}\left(\mathbb{P}^{n}, d\right)$. For example, in the case of the first diagram in Figure 6,

$$
\begin{gathered}
\sigma(\widetilde{\Gamma})=(7 ;\{2\},\{1,3\}), \quad \widetilde{\mathcal{M}}_{\widetilde{\Gamma} ; P}=\widetilde{\mathcal{M}}_{1,([7],\{2\})}, \\
\overline{\mathcal{Z}}_{\widetilde{\Gamma} ; B}=\left(\overline{\mathcal{M}}_{0,3} \times \overline{\mathcal{M}}_{0,2}^{6} \times \overline{\mathcal{M}}_{0,1}^{5}\right) \times\left(\overline{\mathcal{M}}_{0,3}^{2} \times \overline{\mathcal{M}}_{0,2}^{4} \times \overline{\mathcal{M}}_{0,1}^{3}\right) \approx\{\mathrm{pt}\} ; \\
\operatorname{rk} F_{\widetilde{\Gamma} ; B}=\operatorname{rk} F_{\widetilde{\Gamma} ; B}^{\prime}=3, \quad \widetilde{\mathcal{Z}}_{\widetilde{\Gamma} ; B} \approx \mathbb{P}^{2}, \quad \widetilde{\mathcal{Z}}_{\widetilde{\Gamma}} \approx \widetilde{\mathcal{M}}_{1,([7],\{2\})} \times \mathbb{P}^{2} .
\end{gathered}
$$

The weight of the $G$-action on the line $L_{e ; 0}$ is $1 / 2$ of the weight of the $G$-action on $T_{p_{0}} \mathbb{P}_{p_{0}, p_{1}}^{1}[10$, Sects 27.1 and 27.2].

We next describe the equivariant normal bundle $\mathcal{N} \widetilde{\mathcal{Z}}_{\widetilde{\Gamma}}$ of $\widetilde{\mathcal{Z}}_{\widetilde{\Gamma}}$ in $\widetilde{\mathfrak{M}}_{1, k}^{0}\left(\mathbb{P}^{n}, d\right)$. Let

$$
\mathcal{N}_{\overline{\mathfrak{M}}_{\sigma(\widetilde{\Gamma}) ; B}\left(\mathbb{P}^{n}, d\right)} \overline{\mathcal{Z}}_{\widetilde{\Gamma} ; B} \longrightarrow \overline{\mathcal{Z}}_{\widetilde{\Gamma} ; B}
$$

be the normal bundle of $\overline{\mathcal{Z}}_{\widetilde{\Gamma} ; B}$ in $\overline{\mathfrak{M}}_{\sigma(\widetilde{\Gamma}) ; B}\left(\mathbb{P}^{n}, d\right)$. This normal bundle can easily be described using [10, Section 27.4]. Let

$$
F_{\tilde{\Gamma} ; B}^{-}=F_{\sigma(\tilde{\Gamma}) ; B} /\left(F_{\tilde{\Gamma} ; B}^{\prime} \otimes L_{e ; 0}\right) \approx \underset{e^{\prime} \in \operatorname{Edg}\left(v_{0}\right)-\mathrm{Edg}^{+}}{\bigoplus L_{e^{\prime} ; 0}} \oplus \begin{cases}L_{e ; 0}, & \text { if } \mathfrak{d}_{+}(\tilde{\Gamma})=1 ; \\ \{0\}, & \text { if } \mathfrak{d}_{+}(\widetilde{\Gamma}) \geq 2,\end{cases}
$$

where $e$ is an element of $\operatorname{Edg}_{+}$. The normal bundle of $\widetilde{\mathcal{Z}}_{\widetilde{\Gamma} ; B}$ in $\widetilde{\mathfrak{M}}_{\sigma(\widetilde{\Gamma}) ; B}\left(\mathbb{P}^{n}, d\right)$ is given by

$$
\mathcal{N}_{\widetilde{\mathfrak{M}}_{\sigma(\widetilde{\Gamma}) ; B}\left(\mathbb{P}^{n}, d\right)} \widetilde{\mathcal{Z}}_{\widetilde{\Gamma} ; B}=\mathcal{N}_{\overline{\mathfrak{M}}_{\sigma(\widetilde{\Gamma}) ; B}\left(\mathbb{P}^{n}, d\right)} \overline{\mathcal{Z}}_{\widetilde{\Gamma} ; B} \oplus \gamma_{\operatorname{dim}+\widetilde{\Gamma}}^{*} \otimes L_{e ; 0}^{*} \otimes F_{\widetilde{\Gamma} ; B}^{-},
$$

where $\gamma_{\operatorname{dim}_{+} \tilde{\Gamma}} \longrightarrow \mathbb{P}^{\operatorname{dim}} \widetilde{\Gamma}_{+} \tilde{\Gamma}$ is the tautological line bundle. Since none of the exceptional divisors intersects $\widetilde{\mathcal{Z}}_{\widetilde{\Gamma} ; B}$,

$$
\left.\mathbb{E}_{\sigma ; B}\right|_{\widetilde{\mathcal{Z}}_{\widetilde{\Gamma} ; B}}=\gamma_{\operatorname{dim}_{+} \widetilde{\Gamma}^{\otimes}}^{\otimes L_{e ; 0}}
$$

Since the section $\widetilde{\mathcal{D}}_{\sigma ; B}$ is transverse to the zero set, the normal bundle of $\widetilde{\mathcal{Z}}_{\widetilde{\Gamma} ; B}$ in $\widetilde{\mathcal{Z}}_{\sigma(\widetilde{\Gamma}) ; B}\left(\mathbb{P}^{n}, d\right)$ is

$$
\mathcal{N}_{\widetilde{\mathcal{Z}}_{\sigma(\widetilde{\Gamma}) ; B}\left(\mathbb{P}^{n}, d\right)} \widetilde{\mathcal{Z}}_{\widetilde{\Gamma} ; B}=\mathcal{N}_{\widetilde{\mathfrak{M}}_{\sigma(\widetilde{\Gamma}) ; B}\left(\mathbb{P}^{n}, d\right)} \widetilde{\mathcal{Z}}_{\widetilde{\Gamma} ; B} /\left(\gamma_{\operatorname{dim}+\widetilde{\Gamma}}^{*} \otimes L_{e ; 0}^{*} \otimes T_{\mu\left(v_{0}\right)} \mathbb{P}^{n}\right)
$$

by (1-6) and (1-7). Finally,

$$
\mathcal{N} \widetilde{\mathcal{Z}}_{\widetilde{\Gamma}}=\mathcal{N}_{\widetilde{\mathcal{Z}}_{\sigma(\widetilde{\Gamma}) ; B}\left(\mathbb{P}^{n}, d\right)} \widetilde{\mathcal{Z}}_{\widetilde{\Gamma} ; B} \oplus \mathbb{L}_{\sigma(\widetilde{\Gamma}) ; P} \otimes \gamma_{\operatorname{dim}_{+} \widetilde{\Gamma}} \otimes L_{e ; 0}
$$

since the normal bundle of $\widetilde{\mathcal{Z}}_{\sigma(\widetilde{\Gamma})}\left(\mathbb{P}^{n}, d\right)$ in $\widetilde{\mathfrak{M}}_{1, k}^{0}\left(\mathbb{P}^{n}, d\right)$ is $\mathbb{L}_{\sigma(\widetilde{\Gamma}) ; P} \otimes \gamma_{\sigma(\widetilde{\Gamma}) ; B}$. 
In order to compute the last number in (1-4), we also need to determine the restriction of the vector bundle $\widetilde{\mathcal{V}}_{1, k}^{d}$ to $\widetilde{\mathcal{Z}}_{\widetilde{\Gamma}}$. By Theorem 1.2 and (1-9), there is a short exact sequence of vector bundles

$$
\left.\left.0 \longrightarrow \widetilde{\mathcal{V}}_{1, k}^{d}\right|_{\widetilde{\mathcal{Z}}_{\widetilde{\Gamma}}} \longrightarrow \mathcal{V}_{\sigma(\widetilde{\Gamma}) ; B}^{d}\right|_{\widetilde{\mathcal{Z}}_{\widetilde{\Gamma} ; B}} \longrightarrow \gamma_{\operatorname{dim}_{+} \widetilde{\Gamma}}^{*} \otimes L_{e ; 0}^{*} \otimes \mathcal{L}_{\mu\left(v_{0}\right)} \longrightarrow 0
$$

over $\widetilde{\mathcal{Z}}_{\widetilde{\Gamma}}$. This exact sequence describes the euler class of the restriction of $\widetilde{\mathcal{V}}_{1, k}^{d}$ to $\widetilde{\mathcal{Z}}_{\widetilde{\Gamma}}$. If $\sigma=\left(m ; J_{P}, J_{B}\right) \in \mathcal{A}_{1}(d, k)$,

$$
\left\langle c_{1}^{|m|+\left|J_{P}\right|}\left(\mathbb{L}_{\sigma ; P}^{*}\right), \widetilde{\mathcal{M}}_{\sigma ; P}\right\rangle=\frac{m^{\left|J_{P}\right|} \cdot(m-1) !}{24}
$$

by [27, Corollary 1.2]. This is the only intersection number on $\widetilde{\mathcal{M}}_{\sigma ; P}$ needed for computing the last number in (1-4) and the integrals of the cohomology classes on $\overline{\mathfrak{M}}_{1, k}^{0}\left(\mathbb{P}^{n}, d\right)$ that count elliptic curves in $\mathbb{P}^{n}$ passing through specified constraints. For more general enumerative problems, such as counting curves with tangency conditions and with singularities considered by the first author in [18] and the second author in [23], respectively, one would need to compute the intersection numbers of the form

$$
\left\langle c_{1}^{\beta_{0}}\left(\mathbb{L}_{\sigma ; P}^{*}\right) \cdot \prod_{l \in J_{P}} \psi_{l}^{\beta_{l}}, \widetilde{\mathcal{M}}_{\sigma ; P}\right\rangle, \quad \text { where } \quad \beta_{0}+\sum_{l \in J_{P}} \beta_{l}=|m|+\left|J_{P}\right| .
$$

Theorem 1.1 in [27] gives a recursive formula for such numbers. The recursion is on $|m|+\left|J_{P}\right|$, ie the total number of marked points. The starting data for the recursion is the well-known number $\left\langle\psi_{1}, \overline{\mathcal{M}}_{1,1}\right\rangle=1 / 24$.

In the above discussion we ignored all automorphism groups. As in [10, Chapter 27], the rational function for each refined decorated rooted tree $\widetilde{\Gamma}$ obtained following the above algorithm and applying the localization theorem of [1] should be divided by the order of the appropriate automorphism group $\mathbb{A}_{\tilde{\Gamma}}$ :

$$
\left|\mathbb{A}_{\tilde{\Gamma}}\right|=|\operatorname{Aut}(\tilde{\Gamma})| \cdot \prod_{e \in \operatorname{Edg}-\operatorname{Edg}_{0}} \mathfrak{d}(e) .
$$

For example, in the case of the first diagram in Figure 6,

$$
\left|\mathbb{A}_{\tilde{\Gamma}}\right|=1 \cdot\left(1^{3} \cdot 2^{5} \cdot 3^{3}\right)=864
$$

\section{Blowups of moduli spaces of curves}

\subsection{Blowups and subvarieties}

In this section we construct blowups of certain moduli spaces of genus-one and genuszero curves; see Sections 2.3 and 2.4. The former appear in Section 4.3 as the first 
factor in the domain of the proper transforms of the immersion $\iota_{\sigma}$ of Section 1.2. The latter play the analogous role in Section 3.4, where we blow up certain moduli spaces of genus-zero maps. In turn, these last blowups describe the second factor of the domain of maps induced by $\iota_{\sigma}$ in Section 4.3; see Section 1.3 for more details.

We begin by introducing convenient terminology and reviewing standard facts from algebraic geometry. If $\overline{\mathcal{M}}$ is a smooth variety and $Z$ is a smooth subvariety of $\overline{\mathcal{M}}$, let

$$
\left.\mathcal{N}_{\overline{\mathcal{M}}} Z \equiv T \overline{\mathcal{M}}\right|_{Z} / T Z
$$

be the normal bundle of $Z$ in $\overline{\mathcal{M}}$. We denote by

$$
\pi_{Z}^{\perp}:\left.T \overline{\mathcal{M}}\right|_{Z} \longrightarrow \mathcal{N}_{\overline{\mathcal{M}}} Z
$$

the quotient projection map.

Definition 2.1 Let $\overline{\mathcal{M}}$ be a smooth variety.

(1) Smooth subvarieties $X$ and $Y$ of $\overline{\mathcal{M}}$ intersect properly if $X \cap Y$ is a smooth subvariety of $\overline{\mathcal{M}}$ and

$$
T(X \cap Y)=\left.\left.T X\right|_{X \cap Y} \cap T Y\right|_{X \cap Y \cdot{ }^{6}}
$$

(2) If $Z$ is a smooth subvariety of $\overline{\mathcal{M}}$, properly intersecting subvarieties $X$ and $Y$ of $\overline{\mathcal{M}}$ intersect properly relative to $Z$ if

$$
\pi_{Z}^{\perp}\left(\left.T(X \cap Y)\right|_{X \cap Y \cap Z}\right)=\pi_{Z}^{\perp}\left(\left.T X\right|_{X \cap Y \cap Z}\right) \cap \pi_{Z}^{\perp}\left(\left.T Y\right|_{X \cap Y \cap Z}\right) \subset \mathcal{N}_{\overline{\mathcal{M}}} Z .
$$

For example, if $X$ and $Y$ are two smooth curves in a projective space that intersect without being tangent to each other, then $X$ and $Y$ intersect properly (but not transversally, unless the dimension of the projective space is 2). If $X, Y$, and $Z$ are three distinct concurrent lines that lie in a plane, then they intersect properly pairwise, but $X$ and $Y$ do not intersect properly relative to $Z$.

Definition 2.2 If $\overline{\mathcal{M}}$ is a smooth variety, a collection $\left\{\overline{\mathcal{M}}_{\rho}\right\}_{\rho \in \mathcal{A}}$ of smooth subvarieties is properly intersecting if $\overline{\mathcal{M}}_{\rho_{1}}$ and $\overline{\mathcal{M}}_{\rho_{2}}$ intersect properly relative to $\overline{\mathcal{M}}_{\rho_{3}}$ for all $\rho_{1}, \rho_{2}, \rho_{3} \in \mathcal{A}$.

\footnotetext{
${ }^{6}$ In other words, the scheme-theoretic intersection of $X$ and $Y$ is smooth. If the set-theoretic intersection $X \cap Y$ is smooth, the second part of this condition is also equivalent to the injectivity of the natural homomorphism

$$
\left.T X\right|_{X \cap Y} / T(X \cap Y) \longrightarrow T \overline{\mathcal{M}} / T Y
$$
}


If $Z$ is a smooth subvariety of $\overline{\mathcal{M}}$, let

$$
\pi: \mathrm{Bl}_{Z} \overline{\mathcal{M}} \longrightarrow \overline{\mathcal{M}}
$$

be the blowup of $\overline{\mathcal{M}}$ along $Z$. If $X$ is a subvariety of $\overline{\mathcal{M}}$, we denote by $\operatorname{Pr}_{Z} X$ the proper transform of $X$ in $\mathrm{Bl}_{Z} \overline{\mathcal{M}}$, ie the closure of $\pi^{-1}(X-Z)$ in $\mathrm{Bl}_{Z} \overline{\mathcal{M}}{ }^{7}$ The next lemma follows from a local computation. (The local geometry of a proper intersection is particularly simple.)

Lemma 2.3 Let $\overline{\mathcal{M}}$ be a smooth variety.

(1) If $X$ and $Z$ are properly intersecting subvarieties of $\overline{\mathcal{M}}$, then $\operatorname{Pr}_{Z} X$ is a smooth subvariety of $\mathrm{Bl}_{Z} \overline{\mathcal{M}}$ and

$$
\operatorname{Pr}_{Z} X=\mathrm{Bl}_{X \cap Z} X \text {. }
$$

(2) If $X, Y$, and $Z$ are pairwise properly intersecting subvarieties of $\overline{\mathcal{M}}$ and $X$ and $Y$ intersect properly relative to $Z$, then $\operatorname{Pr}_{Z} X$ and $\operatorname{Pr}_{Z} Y$ are properly intersecting subvarieties of $\operatorname{Pr}_{Z} \overline{\mathcal{M}}$ and

$$
\operatorname{Pr}_{Z} X \cap \operatorname{Pr}_{Z} Y=\operatorname{Pr}_{Z}(X \cap Y) .
$$

(3) If $X, Y, Z$, and $Z^{\prime}$ are pairwise properly intersecting subvarieties of $\overline{\mathcal{M}}$ and $X$ and $Y$ intersect properly relative to $Z$ and $Z^{\prime}$, then $\operatorname{Pr}_{Z} X$ and $\operatorname{Pr}_{Z} Y$ intersect properly relative to $\operatorname{Pr}_{Z} Z^{\prime}$.

Corollary 2.4 If $\overline{\mathcal{M}}$ is a smooth variety, $\left\{\overline{\mathcal{M}}_{\rho}\right\}_{\rho \in \mathcal{A}}$ is a properly intersecting collection of subvarieties of $\overline{\mathcal{M}}$, and $\rho \in \mathcal{A}$, then $\left\{\operatorname{Pr}_{\overline{\mathcal{M}}} \overline{\mathcal{M}}_{\rho^{\prime}}\right\}_{\rho^{\prime} \in \mathcal{A}-\{\rho\}}$ is a properly intersecting collection of subvarieties of $\mathrm{Bl}_{\overline{\mathcal{M}}_{\rho}} \overline{\mathcal{M}}$.

Remark By our definitions, properly intersecting subvarieties are necessarily smooth subvarieties of smooth varieties.

\subsection{Moduli spaces of genus-one and -zero curves}

In this subsection, we describe natural subvarieties of moduli spaces of genus-one and -zero curves and natural bundle sections over these moduli spaces. These bundle sections and their twisted versions introduced in the next two subsections are used in Sections 3.4 and 4.3 to describe the structure of the proper transforms of $\overline{\mathfrak{M}}_{1, k}^{0}\left(\mathbb{P}^{n}, d\right)$. Below we also state the now standard facts about these objects that are used in the next two subsections.

\footnotetext{
${ }^{7}$ For the purposes of these definitions we do not require that $\overline{\mathcal{M}}$ and $X$ be smooth.
} 
If $I$ is a finite set, let

$$
\begin{aligned}
& \mathcal{A}_{1}(I)=\left\{\left(I_{P},\left\{I_{k}: k \in K\right\}\right): K \neq \varnothing ; I=\bigsqcup_{k \in\{P\} \sqcup K} I_{k} ;\left|I_{k}\right| \geq 2 \forall k \in K\right\} ; \\
& \mathcal{A}_{0}(I)=\left\{\left(I_{P},\left\{I_{k}: k \in K\right\}\right): K \neq \varnothing ; I=\bigsqcup_{k \in\{P\} \sqcup K} I_{k} ;\left|I_{k}\right| \geq 2 \forall k \in K ;\right. \\
& \left.|K|+\left|I_{P}\right| \geq 2\right\} .
\end{aligned}
$$

If $\rho=\left(I_{P},\left\{I_{k}: k \in K\right\}\right)$ is an element of $\{(I, \varnothing)\} \sqcup \mathcal{A}_{1}(I)$, we denote by $\mathcal{M}_{1, \rho}$ the subset of $\overline{\mathcal{M}}_{1, I}$ consisting of the stable curves $\mathcal{C}$ such that

(i) $\mathcal{C}$ is a union of a smooth torus and $|K|$ projective lines, indexed by $K$;

(ii) each line is attached directly to the torus;

(iii) the marked points on the line corresponding to $k \in K$ are indexed by $I_{k}$.

Let $\overline{\mathcal{M}}_{1, \rho}$ be the closure of $\mathcal{M}_{1, \rho}$ in $\overline{\mathcal{M}}_{1, I}$. Figure 7 illustrates this definition, from the points of view of symplectic topology and of algebraic geometry. In the first diagram, each circle represents a sphere, or $\mathbb{P}^{1}$. In the second diagram, the irreducible components of $\mathcal{C}$ are represented by curves, and the integer next to each component shows its genus. Similarly, if

$$
\rho=\left(I_{P},\left\{I_{k}: k \in K\right\}\right) \in\{(I, \varnothing)\} \sqcup \mathcal{A}_{0}(I),
$$

let $\mathcal{M}_{0, \rho}$ be the subset of $\overline{\mathcal{M}}_{0,\{0\} \sqcup I}$ consisting of the stable curves $\mathcal{C}$ such that

(i) the components of $\mathcal{C}$ are indexed by the set $\{P\} \sqcup K$;

(ii) for each $k \in K$, the component $\mathcal{C}_{k}$ of $\mathcal{C}$ is attached directly to $\mathcal{C}_{P}$;

(iii) for each $k \in K$, the marked points on $\mathcal{C}_{k}$ are indexed by $I_{k}$.

We denote by $\overline{\mathcal{M}}_{0, \rho}$ the closure of $\mathcal{M}_{0, \rho}$ in $\overline{\mathcal{M}}_{0,\{0\} \sqcup I}$. This definition is illustrated in Figure 8. In this case, we do not indicate the genus of the irreducible components in the second diagram, as all of the curves are rational.

The next lemma follows from the fact that for any nodal curve, the deformations of the nodes are independent. More precisely, in the dual to the first-order deformation space of a nodal curve, the vectors corresponding to the smoothings of each node are linearly independent.

Lemma 2.5 If $g=0,1$ and $I$ is a finite set, the collection $\left\{\overline{\mathcal{M}}_{g, \rho}\right\}_{\rho \in \mathcal{A}_{g}(I)}$ is properly intersecting. 

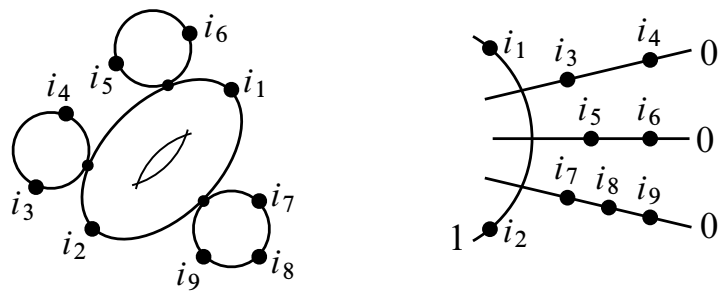

$$
\begin{aligned}
& I_{P}=\left\{i_{1}, i_{2}\right\} \\
& K=\{1,2,3\} \\
& I_{1}=\left\{i_{3}, i_{4}\right\} \\
& I_{2}=\left\{i_{5}, i_{6}\right\} \\
& I_{3}=\left\{i_{7}, i_{8}, i_{9}\right\}
\end{aligned}
$$

Figure 7: A typical element of $\overline{\mathcal{M}}_{1, \rho}$
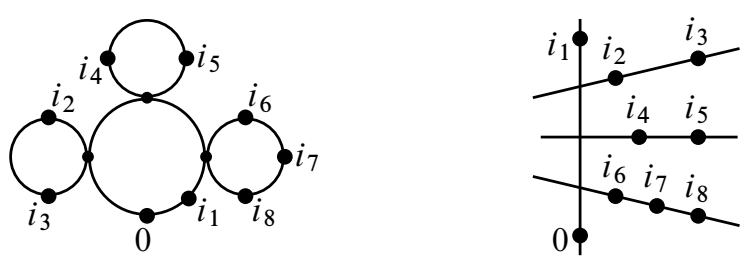

$$
\begin{aligned}
& I_{P}=\left\{i_{1}\right\} \\
& K=\{1,2,3\} \\
& I_{1}=\left\{i_{2}, i_{3}\right\} \\
& I_{2}=\left\{i_{4}, i_{5}\right\} \\
& I_{3}=\left\{i_{6}, i_{7}, i_{8}\right\}
\end{aligned}
$$

Figure 8: A typical element of $\overline{\mathcal{M}}_{0, \rho}$

We define a partial ordering on the sets $\mathcal{A}_{g}(I)$ for $g=0,1$ by setting

$$
\rho^{\prime} \equiv\left(I_{P}^{\prime},\left\{I_{k}^{\prime}: k \in K^{\prime}\right\}\right) \prec \rho \equiv\left(I_{P},\left\{I_{k}: k \in K\right\}\right)
$$

if $\rho^{\prime} \neq \rho$ and there exists a map $\varphi: K \longrightarrow K^{\prime}$ such that $I_{k} \subset I_{\varphi(k)}^{\prime}$ for all $k \in K$. This condition means that the elements of $\mathcal{M}_{\rho^{\prime}}$ can be obtained from the elements of $\mathcal{M}_{\rho}$ by moving more points onto the bubble components or combining the bubble components; see Figure 9. In the $g=0$ case, we define the bubble components to be the components not containing the marked point 0 .
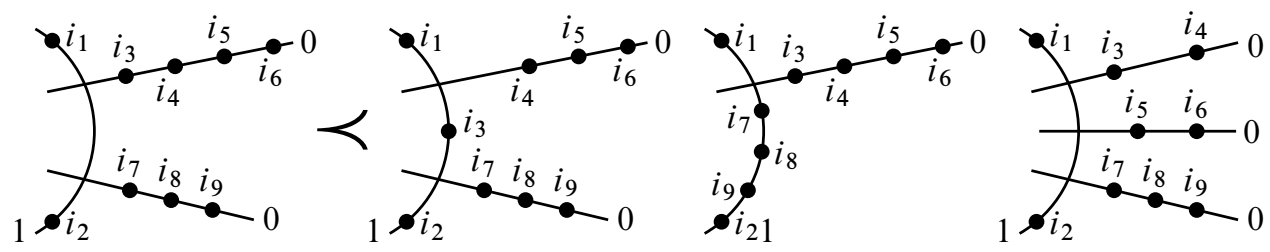

Figure 9: Examples of partial ordering (2-2)

In the blowup constructions of the next two subsections we will twist certain line bundles over moduli spaces of curves and homomorphisms between them. In the rest of this subsection we describe the relevant starting data.

For each $i \in I$, let $L_{i} \longrightarrow \overline{\mathcal{M}}_{1, I}$ be the universal tangent line bundle at the marked point labeled $i$. Let $\mathbb{E} \longrightarrow \overline{\mathcal{M}}_{1, I}$ be the Hodge line bundle of holomorphic differentials. 
The natural pairing of tangent vectors with cotangent vectors induces a section

$$
\begin{aligned}
& \qquad s_{i} \in \Gamma\left(\overline{\mathcal{M}}_{1, I} ; \operatorname{Hom}\left(L_{i}, \mathbb{E}^{*}\right)\right) \\
& \text { Explicitly, } \\
& \text { if } \quad\left[s_{i}([\mathcal{C} ; w])\right\}([\mathcal{C}, \psi])=\psi_{x_{i}(\mathcal{C})} w \\
&
\end{aligned}
$$

and $x_{i}(\mathcal{C}) \in \mathcal{C}$ is the marked point on $\mathcal{C}$ labeled by $i$.

In the genus-zero case, the line bundle $L_{0} \longrightarrow \overline{\mathcal{M}}_{0,\{0\} \sqcup I}$ will be one of the substitutes for $\mathbb{E}$. We note that for every $p \in \mathbb{P}^{1}$, there is a natural isomorphism between the tangent space $T_{p} \mathbb{P}^{1}$ of $\mathbb{P}^{1}$ at $p$ and the space of holomorphic differentials $H^{0}\left(\mathbb{P}^{1} ; T^{*} \mathbb{P}^{1} \otimes\right.$ $\mathcal{O}(2 p)$ ) on $\mathbb{P}^{1}$ that have a pole of order two at $p$. More precisely, let $w$ be a meromorphic function on $\mathbb{P}^{1}$ such that $p$ is the only zero of $w$ and this zero is a simple one. We can then view $w$ as a coordinate around $p$ in $\mathbb{P}^{1}$. Every tangent vector $v \in T_{p} \mathbb{P}^{1}$ can be written as

$$
v=c_{w}(v) \frac{\partial}{\partial w}, \quad c_{w}(v) \in \mathbb{C} .
$$

We define the isomorphism

$$
\psi: T_{p} \mathbb{P}^{1} \longrightarrow H^{0}\left(\mathbb{P}^{1} ; T^{*} \mathbb{P}^{1} \otimes \mathcal{O}(2 p)\right) \quad \text { by } \quad v \longrightarrow \psi_{v}=\frac{c_{w}(v) d w}{w^{2}} .
$$

If $w^{\prime}$ is another meromorphic function on $\mathbb{P}^{1}$ such that $p$ is the only zero of $w^{\prime}$ and this zero is a simple one, then

$$
\begin{array}{cll}
w^{\prime}=\frac{w}{\alpha w+\beta} & \Longrightarrow & d w^{\prime}=\frac{\beta d w}{(\alpha w+\beta)^{2}}, c_{w^{\prime}}(v)=\frac{c_{w}(v)}{\beta} \\
& \Longrightarrow \quad \frac{c_{w^{\prime}}(v) d w^{\prime}}{w^{\prime 2}}=\frac{c_{w}(v) d w}{w^{2}}
\end{array}
$$

Thus, the isomorphism $\psi$ is well-defined. If $i \in I$, we define the section

$$
\begin{aligned}
& s_{i} \in \Gamma\left(\overline{\mathcal{M}}_{0,\{0\} \sqcup I} ; \operatorname{Hom}\left(L_{i}, L_{0}^{*}\right)\right) \quad \text { by } \quad\left\{s_{i}([\mathcal{C} ; w])\right\}([\mathcal{C}, v])=\left.\psi_{v}\right|_{x_{i}(\mathcal{C})} w \\
& \text { if } \quad[\mathcal{C}] \in \overline{\mathcal{M}}_{0,\{0\} \sqcup I},\left.\quad[\mathcal{C}, w] \in L_{i}\right|_{\mathcal{C}}=T_{x_{i}(\mathcal{C})} \mathcal{C},\left.\quad[\mathcal{C}, v] \in L_{0}\right|_{\mathcal{C}}=T_{x_{0}(\mathcal{C})} \mathcal{C} .
\end{aligned}
$$

We note that in both cases the section $s_{i}$ vanishes precisely on the curves for which the point $i$ lies on a bubble component. In fact,

(2-4) $s_{i}^{-1}(0)=\sum_{\rho \in \mathcal{B}_{g}(I ; i)} \overline{\mathcal{M}}_{1, \rho}, \quad$ where $\quad \mathcal{B}_{g}(I ; i)=\left\{\left(I_{P},\left\{I_{B}\right\}\right) \in \mathcal{A}_{g}(I): i \in I_{B}\right\}$. 


\subsection{A blowup of a moduli space of genus-one curves}

Let $I$ and $J$ be finite sets such that $I$ is nonempty. In this subsection, we construct a blowup

$$
\pi_{1,(I, J)}: \widetilde{\mathcal{M}}_{1,(I, J)} \longrightarrow \overline{\mathcal{M}}_{1, I \sqcup J}
$$

of the moduli space $\overline{\mathcal{M}}_{1, I \sqcup J},|I|+1$ line bundles

$$
\widetilde{\mathbb{E}}, \widetilde{L}_{i} \longrightarrow \widetilde{\mathcal{M}}_{1,(I, J)}, \quad i \in I,
$$

and $|I|$ nowhere vanishing sections

$$
\tilde{s}_{i} \in \Gamma\left(\widetilde{\mathcal{M}}_{1,(I, J)} ; \operatorname{Hom}\left(\widetilde{L}_{i}, \widetilde{\mathbb{E}}^{*}\right)\right), \quad i \in I .
$$

Since the sections $\widetilde{s}_{i}$ do not vanish, all $|I|+1$ bundles $\widetilde{L}_{i}$ and $\widetilde{\mathbb{E}}^{*}$ are explicitly isomorphic. They will be denoted by $\mathbb{L}$ and called the universal tangent line bundle.

The smooth variety $\widetilde{\mathcal{M}}_{1,(I, J)}$ is obtained by blowing up some of the subvarieties $\overline{\mathcal{M}}_{1, \rho}$, defined in the previous subsection, and their proper transforms in an order consistent with the partial ordering $\prec$. The line bundle $\widetilde{\mathbb{E}}$ is the sum of the Hodge line bundle $\mathbb{E}$ and all exceptional divisors. For each given $i \in I, \widetilde{L}_{i}$ is the universal tangent line bundle $L_{i}$ for the marked point $i$ minus some of these divisors. The section $\tilde{s}_{i}$ is induced from the pairing $s_{i}$ of the previous subsection.

With $I$ and $J$ as above and $\mathcal{A}_{g}(I \sqcup J)$ as in (2-1), let

$$
\mathcal{A}_{g}(I, J)=\left\{\left(\left(I_{P} \sqcup J_{P}\right),\left\{I_{k} \sqcup J_{k}: k \in K\right\}\right) \in \mathcal{A}_{g}(I \sqcup J): I_{k} \neq \varnothing \forall k \in K\right\} .
$$

We note that if $\rho \in \mathcal{A}_{g}(I \sqcup J)$, then $\rho \in \mathcal{A}_{g}(I, J)$ if and only if every bubble component of an element of $\mathcal{M}_{\rho}$ carries at least one element of $I$. Furthermore,

$$
\mathcal{B}_{g}(I \sqcup J ; i) \subset \mathcal{A}_{g}(I, J) \quad \forall i \in I .
$$

If $|I|+|J| \geq 2$, with respect to the partial ordering $\prec$ the set $\mathcal{A}_{1}(I, J)$ has a unique minimal element:

$$
\rho_{\min } \equiv(\varnothing,\{I \sqcup J\}) .
$$

Let $<$ be an ordering on $\mathcal{A}_{1}(I, J)$ extending the partial ordering $\prec$. We denote the corresponding maximal element by $\rho_{\max }$. If $\rho \in \mathcal{A}_{1}(I, J)$, we put

$$
\rho-1= \begin{cases}\max \left\{\rho^{\prime} \in \mathcal{A}_{1}(I, J): \rho^{\prime}<\rho\right\}, & \text { if } \rho \neq \rho_{\min } \\ 0, & \text { if } \rho=\rho_{\min },\end{cases}
$$

where the maximum is taken with respect to the ordering $<$. 
We now describe the starting data for the inductive blowup procedure involved in constructing the space $\widetilde{\mathcal{M}}_{1,(I, J)}$ and the line bundle $\mathbb{L}$ over $\widetilde{\mathcal{M}}_{1,(I, J)}$. Let

$$
\overline{\mathcal{M}}_{1,(I, J)}^{0}=\overline{\mathcal{M}}_{1, I \sqcup J}, \quad \mathbb{E}_{0}=\mathbb{E} \longrightarrow \overline{\mathcal{M}}_{1,(I, J)}^{0}, \quad \overline{\mathcal{M}}_{1, \rho}^{0}=\overline{\mathcal{M}}_{1, \rho} \forall \rho \in \mathcal{A}_{1}(I, J) .
$$

For each $i \in I$, let

$$
L_{0, i}=L_{i} \longrightarrow \overline{\mathcal{M}}_{1,(I, J)}^{0} \quad \text { and } \quad s_{0, i}=s_{i} \in \Gamma\left(\overline{\mathcal{M}}_{1,(I, J)}^{0} ; \operatorname{Hom}\left(L_{0, i}, \mathbb{E}_{0}^{*}\right)\right) .
$$

By (2-4),

$$
s_{0, i}^{-1}(0)=\sum_{\rho^{*} \in \mathcal{B}_{1}(I \sqcup J ; i)} \overline{\mathcal{M}}_{1, \rho^{*}}^{0}
$$

Suppose $\rho \in \mathcal{A}_{1}(I, J)$ and we have constructed

(I1) a blowup $\pi_{\rho-1}: \overline{\mathcal{M}}_{1,(I, J)}^{\rho-1} \longrightarrow \overline{\mathcal{M}}_{1,(I, J)}^{0}$ of $\overline{\mathcal{M}}_{1,(I, J)}^{0}$ such that $\pi_{\rho-1}$ is an isomorphism outside of the preimages of the spaces $\mathcal{M}_{1, \rho^{\prime}}^{0}$ with $\rho^{\prime} \leq \rho-1$;

(I2) line bundles $L_{\rho-1, i} \longrightarrow \overline{\mathcal{M}}_{1,(I, J)}^{\rho-1}$ for $i \in I$ and $\mathbb{E}_{\rho-1} \longrightarrow \overline{\mathcal{M}}_{1,(I, J)}^{\rho-1}$;

(I3) sections $s_{\rho-1, i} \in \Gamma\left(\overline{\mathcal{M}}_{1,(I, J)}^{\rho-1} ; \operatorname{Hom}\left(L_{\rho-1, i}, \mathbb{E}_{\rho-1}^{*}\right)\right)$ for $i \in I$.

For each $\rho^{*}>\rho-1$, let $\overline{\mathcal{M}}_{1, \rho^{*}}^{\rho-1}$ be the proper transform of $\overline{\mathcal{M}}_{1, \rho^{*}}^{0}$ in $\overline{\mathcal{M}}_{1,(I, J)}^{\rho-1}$. We assume that

(I4) the collection $\left\{\overline{\mathcal{M}}_{1, \rho^{*}}^{\rho-1}\right\}_{\rho^{*} \in \mathcal{A}_{1}(I, J), \rho^{*}>\rho-1}$ is properly intersecting;

(I5) for all $i \in I$,

$$
s_{\rho-1, i}^{-1}(0)=\sum_{\rho^{*} \in \mathcal{B}_{1}(I \sqcup J ; i), \rho^{*}>\rho-1} \overline{\mathcal{M}}_{1, \rho^{*}}^{\rho-1}
$$

The assumption (I5) means that we will gradually be killing the zero locus of the section $s_{i}$. We note that all five assumptions are satisfied if $\rho-1$ is replaced by 0 .

If $\rho$ is as above, let

$$
\tilde{\pi}_{\rho}: \overline{\mathcal{M}}_{1,(I, J)}^{\rho} \longrightarrow \overline{\mathcal{M}}_{1,(I, J)}^{\rho-1}
$$

be the blowup of $\overline{\mathcal{M}}_{1,(I, J)}^{\rho-1}$ along $\overline{\mathcal{M}}_{1, \rho}^{\rho-1}$. We denote by $\overline{\mathcal{M}}_{1, \rho}^{\rho}$ the corresponding exceptional divisor. If $\rho^{*}>\rho$, let $\overline{\mathcal{M}}_{1, \rho^{*}}^{\rho} \subset \overline{\mathcal{M}}_{1,(I, J)}^{\rho}$ be the proper transform of $\overline{\mathcal{M}}_{1, \rho^{*}}^{\rho-1}$. If

$$
\rho=\left(I_{P} \sqcup J_{P},\left\{I_{k} \sqcup J_{k}: k \in K\right\}\right)
$$

and $i \in I$, we put

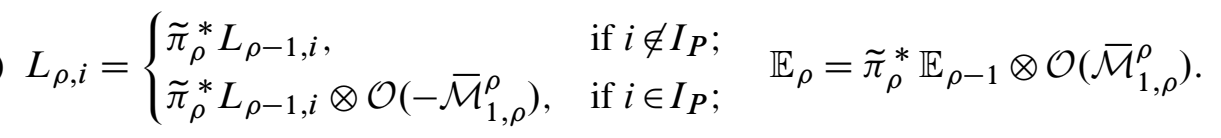


The section $\tilde{\pi}_{\rho}^{*} s_{\rho-1, i}$ induces a section

$$
\tilde{s}_{\rho, i} \in \Gamma\left(\overline{\mathcal{M}}_{1,(I, J)}^{\rho} ; \operatorname{Hom}\left(L_{\rho, i}, \tilde{\pi}_{\rho}^{*} \mathbb{E}_{\rho-1}^{*}\right)\right) .
$$

This section vanishes along $\overline{\mathcal{M}}_{1, \rho}^{\rho}$, by the inductive assumption (I5) if $i \notin I_{P}$. Thus, $\tilde{s}_{\rho, i}$ induces a section

$$
s_{\rho, i} \in \Gamma\left(\overline{\mathcal{M}}_{1,(I, J)}^{\rho} ; \operatorname{Hom}\left(L_{\rho, i}, \mathbb{E}_{\rho}^{*}\right)\right) .
$$

We have now described the inductive step of the procedure. It is immediate that the requirements (I1) $-(I 3)$ and (I5) are satisfied, with $\rho-1$ replaced by $\rho$, are satisfied. Corollary 2.4 and the assumption (I4) imply that the assumption (I4) with $\rho-1$ replaced by $\rho$ is also satisfied.

We conclude the blowup construction after the $\rho_{\max }$ step. Let

$$
\widetilde{\mathcal{M}}_{1,(I, J)}=\overline{\mathcal{M}}_{1,(I, J)}^{\rho_{\max }} ; \quad \widetilde{\mathbb{E}}=\mathbb{E}_{\rho_{\max }} ; \quad \tilde{L}_{i}=L_{\rho_{\max }, i}, \quad \tilde{s}_{i}=s_{\rho_{\max }, i} \quad \forall i \in I .
$$

By (I5), with $\rho-1$ replaced by $\rho_{\max }$, and (2-6), the section $\tilde{s}_{i}$ does not vanish. We note that by $(I 1)$, the stratum

$$
\mathcal{M}_{1,(I, J)} \subset \overline{\mathcal{M}}_{1,(I, J)}
$$

consisting of the smooth curves is a Zariski open subset of $\overline{\mathcal{M}}_{1,(I, J)}^{\rho}$ for all $\rho \in$ $\{0\} \sqcup \mathcal{A}_{1}(I, J)$.

By the next lemma, different extensions of the partial order $\prec$ to an order $<$ on $\mathcal{A}_{1}(I, J)$ correspond to blowing up along disjoint subvarieties in different orders. Thus, the end result of the above blowup construction is well-defined, ie independent of the choice of the ordering $<$ extending the partial ordering $\prec$.

Lemma 2.6 Suppose $\rho, \rho^{\prime} \in \mathcal{A}_{1}(I, J)$ are such that $\rho \nprec \rho^{\prime}$ and $\rho^{\prime} \nprec \rho$. If $\rho \neq \rho^{\prime}$, then the spaces $\overline{\mathcal{M}}_{1, \rho}^{\widetilde{\rho}}$ and $\overline{\mathcal{M}}_{1, \rho^{\prime}}^{\tilde{\rho}}$ are disjoint for some $\tilde{\rho} \prec \rho, \rho^{\prime}$.

Proof (1) Suppose

$$
\rho=\left(I_{P} \sqcup J_{P},\left\{I_{k} \sqcup J_{k}: k \in K\right\}\right) \quad \text { and } \quad \rho^{\prime}=\left(I_{P}^{\prime} \sqcup J_{P}^{\prime},\left\{I_{k}^{\prime} \sqcup J_{k}^{\prime}: k \in K^{\prime}\right\}\right) .
$$

For each $k \in K$ and $k^{\prime} \in K^{\prime}$, let

$$
\begin{aligned}
& \rho_{k}=\left(\left(I-I_{k}\right) \sqcup\left(J-J_{k}\right),\left\{I_{k} \sqcup J_{k}\right\}\right) \in \mathcal{A}_{1}(I, J), \\
& \rho_{k^{\prime}}^{\prime}=\left(\left(I-I_{k^{\prime}}^{\prime}\right) \sqcup\left(J-J_{k^{\prime}}^{\prime}\right),\left\{I_{k^{\prime}}^{\prime} \sqcup J_{k^{\prime}}^{\prime}\right\}\right) \in \mathcal{A}_{1}(I, J) .
\end{aligned}
$$


By definition, $\overline{\mathcal{M}}_{1, \rho_{k}}^{0}$ and $\overline{\mathcal{M}}_{1, \rho_{k^{\prime}}^{\prime}}^{0}$ are divisors in $\overline{\mathcal{M}}_{1,(I, J)}^{0}=\overline{\mathcal{M}}_{1, I \sqcup J}$,

$$
\overline{\mathcal{M}}_{1, \rho}^{0}=\bigcap_{k \in K} \overline{\mathcal{M}}_{1, \rho_{k}}^{0}, \quad \text { and } \quad \overline{\mathcal{M}}_{1, \rho^{\prime}}^{0}=\bigcap_{k^{\prime} \in K^{\prime}} \overline{\mathcal{M}}_{1, \rho_{k^{\prime}}^{\prime}}^{0}
$$

Furthermore, if $\overline{\mathcal{M}}_{1, \rho_{k}}^{0} \cap \overline{\mathcal{M}}_{1, \rho_{k^{\prime}}^{\prime}}^{0} \neq \varnothing$, then either

$$
I_{k} \sqcup J_{k} \subset I_{k^{\prime}}^{\prime} \sqcup J_{k^{\prime}}^{\prime}, \quad I_{k} \sqcup J_{k} \supset I_{k^{\prime}}^{\prime} \sqcup J_{k^{\prime}}^{\prime}, \quad \text { or } \quad\left(I_{k} \sqcup J_{k}\right) \cap\left(I_{k^{\prime}}^{\prime} \sqcup J_{k^{\prime}}^{\prime}\right)=\varnothing \text {. }
$$

(2) Suppose $\overline{\mathcal{M}}_{1, \rho}^{0} \cap \overline{\mathcal{M}}_{1, \rho^{\prime}}^{0} \neq \varnothing$. By the above, there exist decompositions

$$
K=K_{+} \sqcup K_{0} \sqcup \bigsqcup_{l^{\prime} \in K_{+}^{\prime}} K_{l^{\prime}} \quad \text { and } \quad K^{\prime}=K_{+}^{\prime} \sqcup K_{0}^{\prime} \sqcup \bigsqcup_{l \in K_{+}} K_{l}^{\prime}
$$

and a bijection $\varphi: K_{0} \longrightarrow K_{0}^{\prime}$ such that

$$
\begin{gathered}
I_{k} \sqcup J_{k} \subsetneq I_{l^{\prime}}^{\prime} \sqcup J_{l^{\prime}}^{\prime} \quad \forall k \in K_{l^{\prime}}, l^{\prime} \in K_{+}^{\prime}, \quad I_{l} \sqcup J_{l} \supsetneq I_{k^{\prime}}^{\prime} \sqcup J_{k^{\prime}}^{\prime} \quad \forall k^{\prime} \in K_{l}^{\prime}, l \in K_{+}, \\
I_{k} \sqcup J_{k}=I_{\varphi(k)}^{\prime} \sqcup J_{\varphi(k)}^{\prime} \quad \forall k \in K_{0} .
\end{gathered}
$$

We note that the subsets $K_{+}$and $K_{+}^{\prime}$ of $K$ and $K^{\prime}$ are nonempty. For example, if $K_{+}$were empty, then we would have $\rho^{\prime} \prec \rho$, contrary to our assumptions. Let

$$
\widetilde{\rho}=\left(\tilde{I}_{P} \sqcup \widetilde{J}_{P},\left\{\tilde{I}_{k} \sqcup \widetilde{J}_{k}: k \in K_{0} \sqcup K_{+} \sqcup K_{+}^{\prime}\right\}\right) \in \mathcal{A}_{1}(I, J)
$$

be given by

$$
\tilde{I}_{P} \sqcup \widetilde{J}_{P}=\left(I_{P} \cap I_{P}^{\prime}\right) \sqcup\left(J_{P} \cap J_{P}^{\prime}\right), \quad \tilde{I}_{k} \sqcup \widetilde{J}_{k}= \begin{cases}I_{k} \sqcup J_{k}, & \text { if } k \in K_{0} \sqcup K_{+} ; \\ I_{k}^{\prime} \sqcup J_{k}^{\prime}, & \text { if } k \in K_{+}^{\prime} .\end{cases}
$$

For example, if $\rho$ corresponds to the second diagram on the right side of Figure 9 and $\rho^{\prime}$ corresponds to either the first or the third diagram on the right side, then $\tilde{\rho}$ corresponds to the diagram on the left side of Figure 9. By definition, $\widetilde{\rho} \prec \rho, \rho^{\prime}$. Furthermore,

$$
\overline{\mathcal{M}}_{1, \rho}^{0} \cap \overline{\mathcal{M}}_{1, \rho^{\prime}}^{0} \subset \overline{\mathcal{M}}_{1, \tilde{\rho}}^{0}
$$

Thus, by Lemma 2.5, Corollary 2.4, and (2) of Lemma 2.3,

$$
\overline{\mathcal{M}}_{1, \rho}^{\tilde{\rho}} \cap \overline{\mathcal{M}}_{1, \rho^{\prime}}^{\tilde{\rho}} \subset \overline{\mathcal{M}}_{1,(I, J)}^{\tilde{\rho}}
$$

is the closure of the empty set. 


\subsection{A blowup of a moduli space of genus-zero curves}

Suppose $\boldsymbol{\aleph}$ is a nonempty finite set and $\varrho=\left(I_{l}, J_{l}\right)_{l \in \boldsymbol{N}}$ is a tuple of finite sets such that $I_{l} \neq \varnothing$ and $\left|I_{l}\right|+\left|J_{l}\right| \geq 2$ for all $l \in \aleph$. Let

$$
\overline{\mathcal{M}}_{0, \varrho}=\prod_{l \in \mathcal{N}} \overline{\mathcal{M}}_{0,\{0\} \sqcup I_{l} \sqcup J_{l}} \quad \text { and } \quad F_{\varrho}=\bigoplus_{l \in \mathbb{N}} \pi_{l}^{*} L_{0} \longrightarrow \overline{\mathcal{M}}_{0, \varrho},
$$

where $L_{0} \longrightarrow \overline{\mathcal{M}}_{0,\{0\} \sqcup I_{l} \sqcup J_{l}}$ is the universal tangent line bundle for the marked point 0 and

$$
\pi_{l}: \overline{\mathcal{M}}_{0, \varrho} \longrightarrow \overline{\mathcal{M}}_{0,\{0\} \sqcup I_{l} \sqcup J_{l}}
$$

is the projection map. In this subsection, we construct a blowup

$$
\pi_{0, \varrho}: \widetilde{\mathcal{M}}_{0, \varrho} \longrightarrow \mathbb{P} F_{\varrho}
$$

of the projective bundle $\mathbb{P} F_{\varrho}$ over $\overline{\mathcal{M}}_{0, \varrho}$. We also construct line bundles

$$
\widetilde{\mathbb{E}}, \widetilde{L}_{(l, i)} \longrightarrow \widetilde{\mathcal{M}}_{0, \varrho}, \quad i \in I_{l}, l \in \boldsymbol{\aleph},
$$

and nowhere vanishing sections

$$
\tilde{s}_{(l, i)} \in \Gamma\left(\widetilde{\mathcal{M}}_{0, \varrho} ; \operatorname{Hom}\left(\widetilde{L}_{(l, i)}, \widetilde{\mathbb{E}}^{*}\right)\right), \quad i \in I_{l}, l \in \aleph .
$$

In particular, all line bundles $\widetilde{L}_{(l, i)}$ and $\widetilde{\mathbb{E}}^{*}$ are explicitly isomorphic. They will be denoted by $\mathbb{L}$ and called the universal tangent line bundle.

Similarly to the previous subsection, the smooth variety $\widetilde{\mathcal{M}}_{0, \varrho}$ is obtained by blowing up the subvarieties $\overline{\mathcal{M}}_{0, \rho}$ defined below and their proper transforms in an order consistent with a natural partial ordering $\prec$. The line bundle $\widetilde{\mathbb{E}}$ is the sum of the tautological line bundle

$$
\gamma_{\varrho} \longrightarrow \mathbb{P} F_{\varrho}
$$

and all exceptional divisors. For every $l \in \boldsymbol{\aleph}$ and $i \in I_{l}, \widetilde{L}_{(l, i)}$ is $\pi_{l}^{*} L_{i}$ minus some of these divisors. The section $\widetilde{s}_{(l, i)}$ is induced from the pairings $s_{i}$ of Section 2.2.

With $\varrho$ as above and $\mathcal{A}_{0}\left(I_{l}, J_{l}\right)$ as in (2-5), let

$$
\begin{aligned}
& \mathcal{A}_{0}(\varrho)=\left\{\left(\boldsymbol{\aleph}_{+},\left(\rho_{l}\right)_{l \in \boldsymbol{N}}\right): \varnothing \neq \boldsymbol{\aleph}_{+} \subset \boldsymbol{\aleph} ; \rho_{l} \in\left\{\left(I_{l} \sqcup J_{l}, \varnothing\right)\right\} \sqcup \mathcal{A}_{0}\left(I_{l}, J_{l}\right) \forall l \in \boldsymbol{\aleph} ;\right. \\
&\left.\rho_{l}=\left(I_{l} \sqcup J_{l}, \varnothing\right) \forall l \in \boldsymbol{\aleph}-\boldsymbol{\aleph}_{+} ;\left(\boldsymbol{\aleph}_{+},\left(\rho_{l}\right)_{l \in \boldsymbol{N}}\right) \neq\left(\boldsymbol{\aleph},\left(I_{l} \sqcup J_{l}, \varnothing\right)_{l \in \boldsymbol{N}}\right)\right\} .
\end{aligned}
$$

We define a partial ordering on $\mathcal{A}_{0}(\varrho)$ by setting

$$
\rho^{\prime} \equiv\left(\aleph_{+}^{\prime},\left(\rho_{l}^{\prime}\right)_{l \in \boldsymbol{N}}\right) \prec \rho \equiv\left(\boldsymbol{\aleph}_{+},\left(\rho_{l}\right)_{l \in \boldsymbol{N}}\right)
$$

if $\rho^{\prime} \neq \rho, \aleph_{+}^{\prime} \subset \aleph_{+}$, and for every $l \in \boldsymbol{\aleph}$ either $\rho_{l}^{\prime}=\rho_{l}, \rho_{l}^{\prime} \prec \rho_{l}$, or $\rho_{l}^{\prime}=\left(I_{l} \sqcup J_{l}, \varnothing\right)$. Let $<$ be an ordering on $\mathcal{A}_{0}(\varrho)$ extending the partial ordering $\prec$. We denote the corresponding 
minimal and maximal elements of $\mathcal{A}_{0}(\varrho)$ by $\rho_{\min }$ and $\rho_{\max }$, respectively. If $\rho \in \mathcal{A}_{0}(\varrho)$, we define

$$
\rho-1 \in\{0\} \sqcup \mathcal{A}_{0}(\varrho)
$$

as in (2-7).

If $\rho \in \mathcal{A}_{0}(\varrho)$ is as in $(2-11)$, let

$$
\overline{\mathcal{M}}_{0, \rho}=\prod_{l \in \mathcal{K}} \overline{\mathcal{M}}_{0, \rho_{l}}, \quad F_{\rho}=\left.\bigoplus_{l \in \mathcal{N}^{+}} \pi_{l}^{*} L_{0}\right|_{\overline{\mathcal{M}}_{0, \rho}} \subset F_{\varrho},
$$

and

$$
\widetilde{\mathcal{M}}_{0, \rho}^{0}=\mathbb{P} F_{\rho} \subset \widetilde{\mathcal{M}}_{0, \varrho}^{0} \equiv \mathbb{P} F_{\varrho} .
$$

The spaces $\widetilde{\mathcal{M}}_{0, \varrho}^{0}$ and $\widetilde{\mathcal{M}}_{0, \rho}^{0}$ can be represented by diagrams as in Figure 10.

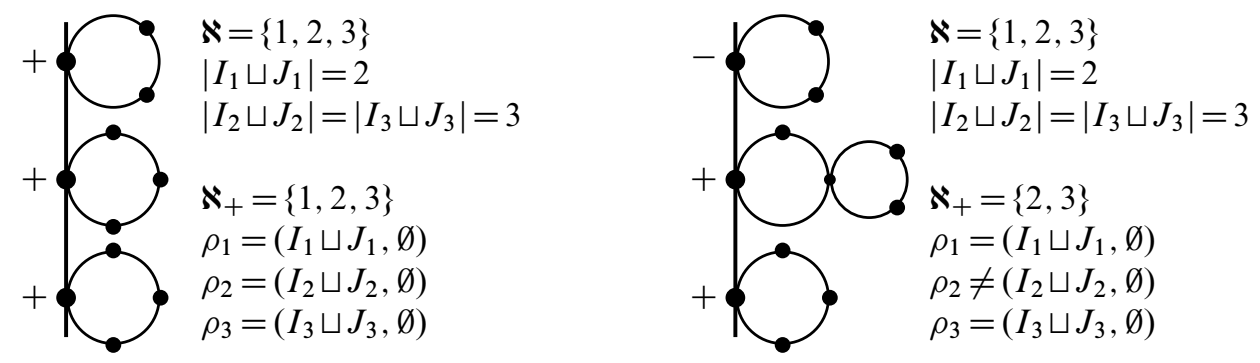

Figure 10: Typical elements of $\widetilde{\mathcal{M}}_{0, \varrho}^{0}$ and $\widetilde{\mathcal{M}}_{0, \rho}^{0}$

The trees of circles attached to the vertical lines correspond to the tuples $\rho_{l}$, with conventions as in the first, symplectic-topology, diagram in Figure 8. For each such tree, the marked point 0 is the point on the line. We indicate the elements of $\aleph_{+} \subset \boldsymbol{\aleph}$ with plus signs next to these points. Note that by (2-10), every dot on a vertical line for which the corresponding tree of circles contains more than one circle must be labeled with a plus sign. From Lemma 2.5, we immediately obtain the following lemma:

Lemma 2.7 Suppose $\boldsymbol{\aleph}$ is a nonempty finite set and $\varrho=\left(I_{l}, J_{l}\right)_{l \in \mathbb{N}}$ is a tuple of finite sets such that $I_{l} \neq \varnothing$ and $\left|I_{l}\right|+\left|J_{l}\right| \geq 2$ for all $l \in \aleph$. If $\mathcal{A}_{0}(\varrho)$ is as above, the collection $\left\{\widetilde{\mathcal{M}}_{0, \rho}^{0}\right\}_{\rho \in \mathcal{A}_{0}(\varrho)}$ is properly intersecting.

We now describe the starting data for the sequential blowup construction of this subsection. Let

$$
\mathbb{E}_{0}=\gamma_{\varrho} \longrightarrow \widetilde{\mathcal{M}}_{0, \varrho}^{0}=\mathbb{P} F_{\varrho} \quad \text { and } \quad L_{0,(l, i)}=\pi_{0, \varrho}^{*} \pi_{l}^{*} L_{i} \longrightarrow \widetilde{\mathcal{M}}_{0, \varrho}^{0} \forall i \in I_{l}, l \in \boldsymbol{N} .
$$

We take

$$
s_{0,(l, i)} \in \Gamma\left(\widetilde{\mathcal{M}}_{0, \varrho}^{0} ; \operatorname{Hom}\left(L_{0,(l, i)}, \mathbb{E}_{0}^{*}\right)\right)
$$


to be the section induced by $\pi_{0, \varrho}^{*} \pi_{l}^{*} s_{i}$, with $s_{i}$ defined by (2-3). It follows immediately from (2-4) that

$$
\underset{0,(l, i)}{-1}(0)=\sum_{\rho^{*} \in \mathcal{B}_{0}(\varrho ; l, i)} \widetilde{\mathcal{M}}_{0, \rho^{*}}^{0},
$$

where $\mathcal{B}_{0}(\varrho ; l, i)=\left\{\left(\aleph_{+},\left(\rho_{l^{\prime}}\right)_{l^{\prime} \in \mathbb{N}}\right) \in \mathcal{A}_{0}(\varrho)\right.$ :

$$
\begin{aligned}
& \aleph_{+}=\boldsymbol{\aleph}-\{l\} \text { and } \rho_{l^{\prime}}=\left(I_{l^{\prime}} \sqcup J_{l^{\prime}}, \varnothing\right) \forall l^{\prime} \in \boldsymbol{N}, \text { or } \\
& \left.\aleph_{+}=\boldsymbol{\aleph}, \rho_{l} \in \mathcal{B}_{0}\left(I_{l} \sqcup J_{l} ; i\right), \rho_{l^{\prime}}=\left(I_{l^{\prime}} \sqcup J_{l^{\prime}}, \varnothing\right) \forall l^{\prime} \in \boldsymbol{\aleph}-\{l\}\right\} .
\end{aligned}
$$

The rest of the construction proceeds as in Section 2.3. The analogue of (2-9) now is

$$
\begin{aligned}
& L_{\rho,(l, i)}=\left\{\begin{array}{lc}
\tilde{\pi}_{\rho}^{*} L_{\rho-1,(l, i)}, & \text { if } l \notin \boldsymbol{N}_{+} \text {or } \\
\tilde{\pi}_{\rho}^{*} L_{\rho-1,(l, i)} \otimes \mathcal{O}\left(-\widetilde{\mathcal{M}}_{0, \rho}^{\rho}\right), & \text { otherwise; }
\end{array}\right. \\
& \mathbb{E}_{\rho}=\tilde{\pi}_{\rho}^{*} \mathbb{E}_{\rho-1} \otimes \mathcal{O}\left(\tilde{\mathcal{M}}_{0, \rho}^{\rho}\right) .
\end{aligned}
$$

As before, we take

$$
\begin{gathered}
\widetilde{\mathcal{M}}_{0, \varrho}=\widetilde{\mathcal{M}}_{0, \varrho}^{\rho_{\max }} ; \quad \widetilde{\mathbb{E}}_{=} \mathbb{E}_{\rho_{\max }} ; \\
\tilde{L}_{(l, i)}=L_{\rho_{\max },(l, i)} \quad \text { and } \quad \tilde{s}_{(l, i)}=s_{\rho_{\max },(l, i)} \quad \forall i \in I_{l}, l \in \mathbb{N} .
\end{gathered}
$$

The analogue of the inductive assumption (I5) insures that each section $\widetilde{s}_{(l, i)}$ does not vanish. The statement and the proof of Lemma 2.6 remain valid in the present setting, with only minor changes. Thus, the end result of the above blowup construction is again well-defined, ie independent of the choice of the ordering $<$ extending the partial ordering $\prec$.

\section{A blowup of a moduli space of genus-zero maps}

\subsection{Blowups and immersions}

In this section we construct blowups of certain moduli spaces of genus-zero maps; see Sections 3.3 and 3.4. As outlined in Section 1.3, these blowups appear in Section 4.3 as the second factor in the domain of the immersions induced by the immersions $\iota_{\sigma}$ of Section 1.2.

As in Section 2, we begin by introducing convenient terminology and reviewing standard facts from algebraic geometry. If $\overline{\mathfrak{M}}$ is a variety, we denote its Zariski tangent space and its tangent cone by $T \overline{\mathfrak{M}}$ and $T C \overline{\mathfrak{M}}$, respectively. If $X$ is a smooth variety (but not 
necessarily equidimensional), we recall that a morphism $\iota_{X}: X \longrightarrow \overline{\mathfrak{M}}$ is an immersion if the differential of $\iota_{X}$,

$$
d \iota_{X}: T X \longrightarrow \iota_{X}^{*} T C \overline{\mathfrak{M}},
$$

is injective at every point of $X$. Let

$$
\operatorname{Im}^{s} \iota_{X} \equiv\left\{p \in \overline{\mathfrak{M}}:\left|\iota_{X}^{-1}(p)\right| \geq 2\right\} \quad \text { and } \quad \mathcal{N}_{\iota_{X}} \equiv \iota_{X}^{*} T C \overline{\mathfrak{M}} / \operatorname{Im} d \iota_{X}
$$

be the singular locus of $\iota_{X}$ and the normal cone of $\iota_{X}$ in $\overline{\mathfrak{M}}$, respectively. We denote by

$$
\pi_{\iota_{X}}^{\perp}: \iota_{X}^{*} T C \overline{\mathfrak{M}} \longrightarrow \mathcal{N}_{\iota_{X}}
$$

the projection map. If $Z$ is a subvariety of $\overline{\mathfrak{M}}$, let

$$
\iota_{Z}: Z \longrightarrow \overline{\mathfrak{M}}
$$

the inclusion map.

Definition 3.1 Let $\overline{\mathfrak{M}}$ be a variety.

(1) An immersion $\iota_{X}: X \longrightarrow \overline{\mathfrak{M}}$ is properly self-intersecting if for all $x_{1}, x_{2} \in X$ such that $\iota_{X}\left(x_{1}\right)=\iota_{X}\left(x_{2}\right)$ and sufficiently small neighborhoods $U_{1}$ of $x_{1}$ and $U_{2}$ of $x_{2}$ in $X$

$$
T C_{\iota_{X}\left(x_{1}\right)}\left(\iota_{X}\left(U_{1}\right) \cap \iota_{X}\left(U_{2}\right)\right)=\left.\left.\operatorname{Im} d \iota_{X}\right|_{x_{1}} \cap \operatorname{Im} d \iota_{X}\right|_{x_{2}} \subset T C_{\iota_{X}\left(x_{1}\right)} \overline{\mathfrak{M}} .{ }^{8}
$$

(2) If $\iota_{X}: X \longrightarrow \overline{\mathfrak{M}}$ and $\iota_{Y}: Y \longrightarrow \overline{\mathfrak{M}}$ are immersions such that $\iota_{X}$ is properly selfintersecting, $\iota_{X}$ is properly self-intersecting relative to $\iota_{Y}$ if for all $x_{1}, x_{2} \in X$ and $y \in Y$ such that

$$
\iota_{X}\left(x_{1}\right)=\iota_{X}\left(x_{2}\right)=\iota_{Y}(y)
$$

and for all sufficiently small neighborhoods $U_{1}$ of $x_{1}$ and $U_{2}$ of $x_{2}$ in $X$,

$$
\left.\pi_{\iota_{Y}}^{\perp}\right|_{y}\left(T C_{\iota_{Y}(y)}\left(\iota_{X}\left(U_{1}\right) \cap \iota_{X}\left(U_{2}\right)\right)\right)=\left.\left.\left.\left.\left.\pi_{\iota_{Y}}^{\perp}\right|_{y} \operatorname{Im} d \iota_{X}\right|_{x_{1}} \cap \pi_{\iota_{Y}}^{\perp}\right|_{y} \operatorname{Im} d \iota_{X}\right|_{x_{2}} \subset \mathcal{N}_{\iota_{Y}}\right|_{y} .
$$

This definition generalizes Definition 2.1; see the paragraph following the latter for some examples.

Definition 3.2 If $\overline{\mathfrak{M}}$ is a variety, a collection $\left\{\iota_{\varrho}: X_{\varrho} \longrightarrow \overline{\mathfrak{M}}\right\}_{\varrho \in \mathcal{A}}$ of immersions is properly self-intersecting if for all $\rho_{1}, \rho_{2}, \rho_{3} \in \mathcal{A}$ the immersion $\iota_{\rho_{1}} \sqcup \iota_{\rho_{2}}$ is properly self-intersecting relative to $\iota_{\rho_{3}}$.

The next lemma follows from a local computation. (The local geometry of a proper self-intersection is particularly simple.)

\footnotetext{
${ }^{8} \mathrm{We}$ emphasize that intersections are taken to be set-theoretic intersections unless otherwise noted.
} 
Lemma 3.3 Suppose $\overline{\mathfrak{M}}$ is a variety and $Z$ is a smooth subvariety of $\overline{\mathfrak{M}}$.

(1) If $\iota_{X}: X \longrightarrow \overline{\mathfrak{M}}$ is an immersion such that the immersion $\iota_{X} \sqcup \iota_{Z}: X \sqcup Z \longrightarrow \overline{\mathfrak{M}}$ is properly self-intersecting, then $\iota_{X}$ lifts to an immersion

$$
\operatorname{Pr}_{Z} \iota_{X}: \mathrm{Bl}_{\iota_{X}^{-1}(Z)} X \longrightarrow \mathrm{Bl}_{Z} \overline{\mathfrak{M}} \quad \text { s.t. } \quad \operatorname{Im} \operatorname{Pr}_{Z} \iota_{X}=\operatorname{Pr}_{Z} \operatorname{Im} \iota_{X} .
$$

(2) If in addition $\iota_{X}$ is properly self-intersecting relative to $\iota_{Z}$, then $\operatorname{Pr}_{Z} \iota_{X}$ is properly self-intersecting and

$$
\operatorname{Im}^{s} \operatorname{Pr}_{Z} \iota_{X}=\operatorname{Pr}_{Z} \operatorname{Im}^{s} \iota \iota_{X}
$$

(3) If in addition $\iota_{Y}: Y \longrightarrow \overline{\mathfrak{M}}$ is an immersion such that $\iota_{X} \sqcup \iota_{Y} \sqcup \iota_{Z}$ is properly selfintersecting and $\iota_{X}$ is properly self-intersecting relative to $\iota_{Y}$, then $\operatorname{Pr}_{Z} \iota_{X}$ is properly self-intersecting relative to $\operatorname{Pr}_{Z} \iota_{Y}$. Furthermore,

$$
\left\{\operatorname{Pr}_{Z} \iota_{X}\right\}^{-1}\left(\operatorname{Pr}_{Z} \operatorname{Im} \iota_{Y}\right)=\operatorname{Pr}_{\iota_{X}^{-1}(Z)} \iota_{X}^{-1}\left(\operatorname{Im} \iota_{Y}\right)
$$

Remark Since we always require that the blowup locus be smooth, an implicit conclusion of (1) of Lemma 3.3 is that $\iota_{X}^{-1}(Z)$ is a smooth subvariety of $X$; this is immediate from the local situation. Note that $X$ itself is smooth, as it is the domain of the immersion $\iota_{X}$.

Corollary 3.4 Suppose $\overline{\mathfrak{M}}$ is a variety and $\left\{\iota_{\varrho}: X_{\varrho} \longrightarrow \overline{\mathfrak{M}}\right\}_{\varrho \in \mathcal{A}}$ is a properly selfintersecting collection of immersions. If $\varrho \in \mathcal{A}$ is such that $\iota_{\varrho}$ is an embedding, then $\left\{\operatorname{Pr}_{\operatorname{Im}} \iota_{\varrho} \iota^{\prime}\right\}_{\varrho^{\prime} \in \mathcal{A}-\{\varrho\}}$ is a properly self-intersecting collection of immersions into $\mathrm{Bl}_{\mathrm{Im} \iota} \overline{\mathfrak{M}}$.

Like Lemma 3.3, the next lemma follows from a local computation, using the simple geometry of a proper self-intersection.

Lemma 3.5 Suppose $\overline{\mathfrak{M}}$ is a smooth variety, $Z$ is a smooth subvariety of $\overline{\mathfrak{M}}$, $\iota_{X}: X \longrightarrow \overline{\mathfrak{M}}$ is an immersion such that the immersion $\iota_{X} \sqcup \iota_{Z}$ is properly selfintersecting. Let

$$
\iota_{X}^{-1}(Z)=\bigsqcup_{\varrho \in \mathcal{A}} Z_{\varrho}
$$

be the decomposition of $\iota_{X}^{-1}(Z)$ into path components. If there exist a splitting

$$
\mathcal{N}_{l_{X}}=\bigoplus_{i \in I} L_{i} \longrightarrow X
$$


and a subset $I_{\varrho}$ of $I$ for each $\varrho \in \mathcal{A}$ such that

$$
\left.\iota_{X}\right|_{Z_{Q}} ^{*} T Z / T Z_{\varrho}=\left.\bigoplus_{i \in I-I_{\varrho}} L_{i}\right|_{Z_{\varrho}} \quad \forall \varrho \in \mathcal{A},
$$

then

$$
\mathcal{N}_{\operatorname{Pr} Z \iota_{X}}=\bigoplus_{i \in I}\left(\pi^{*} L_{i} \otimes \bigotimes_{i \in I_{\varrho}} \mathcal{O}\left(-E_{\varrho}\right)\right)
$$

where $E_{\varrho}$ is the component of the exceptional divisor for the blowup $\pi: \mathrm{Bl}_{l_{X}^{-1}(Z)} X \longrightarrow$ $X$ that projects onto $Z_{\varrho}$.

We note that by (1) of Definition 3.1, the homomorphism

$$
\left.\iota_{X}\right|_{Z_{Q}} ^{*} T Z / T Z_{\varrho} \longrightarrow \mathcal{N}_{\iota_{X}} \equiv \iota_{X}^{*} T \overline{\mathfrak{M}} / \operatorname{Im} d \iota_{X}
$$

induced by the inclusions is injective. Thus, we can identify $\left.\iota_{X}\right|_{Z_{Q}} ^{*} T Z / T Z_{\varrho}$ with a subbundle of $\mathcal{N}_{\iota_{X}}$, as we have done in Lemma 3.5.

\subsection{Moduli spaces of genus-zero maps}

In this subsection, we describe natural subvarieties of the moduli space of genus-zero maps and a natural bundle section over them. This bundle section induces other bundle sections, introduced in the next two subsections, that are used in the blowup construction of Section 4.3 to describe the structure of the proper transforms of $\overline{\mathfrak{M}}_{1, k}^{0}\left(\mathbb{P}^{n}, d\right)$; see Section 1.3 for more details. Below we also state two well-known facts in the GromovWitten theory, Lemmas 3.6 and 3.7, and a more recent result, Lemma 3.8.

If $d \in \mathbb{Z}^{+}$and $J$ is a finite set, let

$$
\begin{gathered}
\mathcal{A}_{0}(d, J)=\left\{\left(m ; J_{P}, J_{B}\right): m \in \mathbb{Z}^{+}, m \leq d ; J=J_{P} \sqcup J_{B}, m+\left|J_{P}\right| \geq 2\right\} ; \\
\overline{\mathfrak{M}}_{0,(0, J)}\left(\mathbb{P}^{n}, d\right)=\overline{\mathfrak{M}}_{0,\{0\} \sqcup J}\left(\mathbb{P}^{n}, d\right) .
\end{gathered}
$$

If $\sigma=\left(m ; J_{P}, J_{B}\right) \in \mathcal{A}_{0}(d, J)$, let $\mathfrak{M}_{0, \sigma}\left(\mathbb{P}^{n}, d\right)$ be the subset of $\overline{\mathfrak{M}}_{0,\{0\} \sqcup J}\left(\mathbb{P}^{n}, d\right)$ consisting of the stable maps $[\Sigma, u]$ such that

(i) the components of $\Sigma$ are $\Sigma_{i}=\mathbb{P}^{1}$ with $i \in\{P\} \sqcup[m]$;

(ii) $\left.u\right|_{\Sigma_{P}}$ is constant and the marked points on $\Sigma_{P}$ are indexed by the set $\{0\} \sqcup J_{P}$;

(iii) for each $i \in[m], \Sigma_{i}$ is attached to $\Sigma_{P}$ and $\left.u\right|_{\Sigma_{i}}$ is not constant.

We denote by $\overline{\mathfrak{M}}_{0, \sigma}\left(\mathbb{P}^{n}, d\right)$ the closure of $\mathfrak{M}_{0, \sigma}\left(\mathbb{P}^{n}, d\right)$ in $\overline{\mathfrak{M}}_{0,\{0\} \sqcup J}\left(\mathbb{P}^{n}, d\right)$. Figure 11 illustrates this definition, from the points of view of symplectic topology and of algebraic geometry. In the first diagram, each disk represents a sphere, and we 
shade the components on which the map $u$ is nonconstant. In the second diagram, the irreducible components of $\Sigma$ are represented by lines, and the integer next to each component shows the degree of $u$ on that component. In both cases, we indicate the marked points lying on the component $\Sigma_{P}$ only.
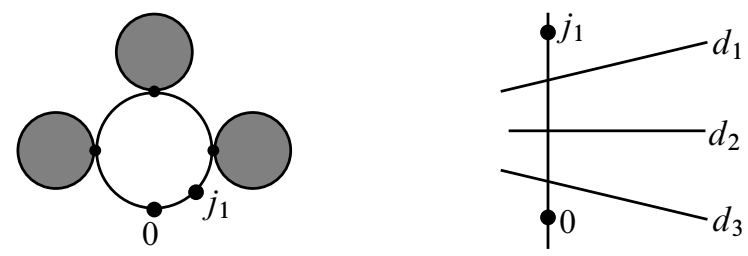

$$
\begin{aligned}
& m=3, J_{P}=\left\{j_{1}\right\} \\
& d_{1}, d_{2}, d_{3}>0 \\
& d_{1}+d_{2}+d_{3}=d
\end{aligned}
$$

Figure 11: A typical element of $\overline{\mathfrak{M}}_{0, \sigma}\left(\mathbb{P}^{n}, d\right)$

We define a partial ordering on the set $\mathcal{A}_{0}(d, J)$ by setting

$$
\sigma^{\prime} \equiv\left(m^{\prime} ; J_{P}^{\prime}, J_{B}^{\prime}\right) \prec \sigma \equiv\left(m ; J_{P}, J_{B}\right) \quad \text { if } \quad \sigma^{\prime} \neq \sigma, m^{\prime} \leq m, J_{P}^{\prime} \subset J_{P} .
$$

Similarly to Section 2.2 , this condition means that the elements of $\mathfrak{M}_{0, \sigma^{\prime}}\left(\mathbb{P}^{n}, d\right)$ can be obtained from the elements of $\mathfrak{M}_{0, \sigma}\left(\mathbb{P}^{n}, d\right)$ by moving more points onto the bubble components or combining the bubble components; see Figure 12. As in the $g=0$ case of Section 2.2, the bubble components are the components not containing the marked point 0 .
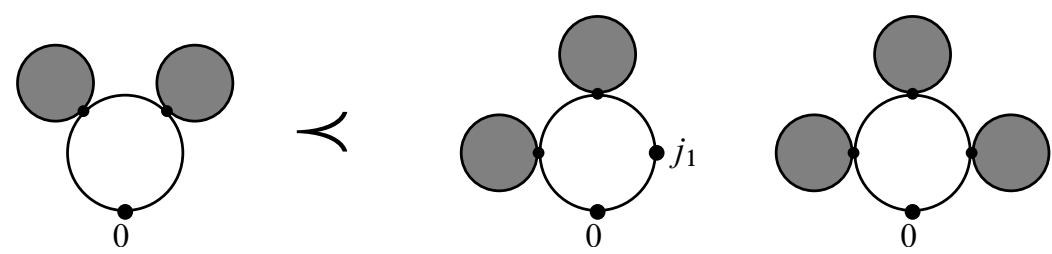

Figure 12: Examples of partial ordering (3-3)

Lemma 3.6 If $\sigma_{1}, \sigma_{2} \in \mathcal{A}_{0}(d, J), \sigma_{1} \neq \sigma_{2} \sigma_{1} \nprec \sigma_{2}$, and $\sigma_{2} \nprec \sigma_{1}$, then

$$
\overline{\mathfrak{M}}_{0, \sigma_{1}}\left(\mathbb{P}^{n}, d\right) \cap \overline{\mathfrak{M}}_{0, \sigma_{2}}\left(\mathbb{P}^{n}, d\right) \subset \overline{\mathfrak{M}}_{0, \tilde{\sigma}\left(\sigma_{1}, \sigma_{2}\right)}\left(\mathbb{P}^{n}, d\right),
$$

where

$$
\tilde{\sigma}\left(\sigma_{1}, \sigma_{2}\right)=\max \left\{\sigma^{\prime} \in \mathcal{A}_{0}(d, J): \sigma^{\prime} \prec \sigma_{1}, \sigma_{2}\right\} .
$$

If $\tilde{\sigma}\left(\sigma_{1}, \sigma_{2}\right)$ is not defined, $\overline{\mathfrak{M}}_{0, \sigma_{1}}\left(\mathbb{P}^{n}, d\right)$ and $\overline{\mathfrak{M}}_{0, \sigma_{2}}\left(\mathbb{P}^{n}, d\right)$ are disjoint.

For example, if $\sigma_{1}$ and $\sigma_{2}$ correspond to the two diagrams on the right side of Figure 12, then $\widetilde{\sigma}\left(\sigma_{1}, \sigma_{2}\right)$ corresponds to the diagram on the left side of Figure 12. Lemma 3.6 is immediate from the definition of the topology on $\overline{\mathfrak{M}}_{0,\{0\} \sqcup J}\left(\mathbb{P}^{n}, d\right)$. It can also 
be easily deduced from [16, Subsection 3.2] by an argument similar to the proof of Lemma 2.6.

If $\sigma=\left(m ; J_{P}, J_{B}\right)$ is an element of $\mathcal{A}_{0}(d, J)$, let

$$
\overline{\mathfrak{M}}_{\sigma ; B}\left(\mathbb{P}^{n}, d\right) \subset \prod_{i \in[m]} \bigsqcup_{d_{i}>0, J_{i} \subset J_{B}} \overline{\mathfrak{M}}_{0,\{0\} \sqcup J_{i}}\left(\mathbb{P}^{n}, d_{i}\right)
$$

and

$$
\pi_{i}: \overline{\mathfrak{M}}_{\sigma ; B}\left(\mathbb{P}^{n}, d\right) \longrightarrow \bigsqcup_{d_{i}>0, J_{i} \subset J_{B}} \overline{\mathfrak{M}}_{0,\{0\} \sqcup J_{i}}\left(\mathbb{P}^{n}, d_{i}\right), \quad i \in[m]
$$

be as in Section 1.2. Since each of the spaces $\overline{\mathfrak{M}}_{0,\{0\} \sqcup J_{i}}\left(\mathbb{P}^{n}, d_{i}\right)$ is smooth and each of the evaluation maps

$$
\mathrm{ev}_{0}: \overline{\mathfrak{M}}_{0,\{0\} \sqcup J_{i}}\left(\mathbb{P}^{n}, d_{i}\right) \longrightarrow \mathbb{P}^{n}
$$

is a submersion, the space $\overline{\mathfrak{M}}_{\sigma ; B}\left(\mathbb{P}^{n}, d\right)$ is smooth. We denote by

$$
\iota_{\sigma}: \overline{\mathcal{M}}_{0,\{0\} \sqcup[m] \sqcup J_{P}} \times \overline{\mathfrak{M}}_{\sigma ; B}\left(\mathbb{P}^{n}, d\right) \longrightarrow \overline{\mathfrak{M}}_{0, \sigma}\left(\mathbb{P}^{n}, d\right) \subset \overline{\mathfrak{M}}_{0,\{0\} \sqcup J}\left(\mathbb{P}^{n}, d\right)
$$

the natural node-identifying map. It descends to an immersion

$$
\bar{\iota}_{\sigma}:\left(\overline{\mathcal{M}}_{0,\{0\} \sqcup[m] \sqcup J_{P}} \times \overline{\mathfrak{M}}_{\sigma ; B}\left(\mathbb{P}^{n}, d\right)\right) / S_{m} \longrightarrow \overline{\mathfrak{M}}_{0,\{0\} \sqcup J}\left(\mathbb{P}^{n}, d\right) .
$$

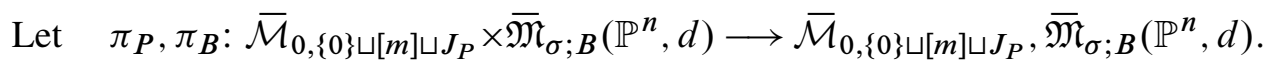

be the natural projection maps.

Lemma 3.7 If $d \in \mathbb{Z}^{+}$and $J$ is a finite set, the collections $\left\{\iota_{\sigma}\right\}_{\sigma \in \mathcal{A}_{0}(d, J)}$ and $\left\{\bar{l}_{\sigma}\right\}_{\sigma \in \mathcal{A}_{0}(d, J)}$ of immersions are properly self-intersecting. If $\sigma \in \mathcal{A}_{0}(d, J)$ is as in (3-3),

$$
\operatorname{Im}^{s} \bar{\iota}_{\sigma} \subset \bigcup_{\sigma^{\prime}<\sigma} \overline{\mathfrak{M}}_{0, \sigma^{\prime}}\left(\mathbb{P}^{n}, d\right) \quad \text { and } \quad \mathcal{N}_{\iota_{\sigma}}=\bigoplus_{i \in[m]} \pi_{P}^{*} L_{i} \otimes \pi_{B}^{*} \pi_{i}^{*} L_{0}
$$

If in addition $\sigma^{\prime} \in \mathcal{A}_{0}(d, J), \sigma^{\prime} \prec \sigma$, and $\sigma^{\prime}$ is as in (3-3), then

$$
\iota_{\sigma}^{-1}\left(\overline{\mathfrak{M}}_{0, \sigma^{\prime}}\left(\mathbb{P}^{n}, d\right)\right)=\left(\bigcup_{\rho \in \mathcal{A}_{0}\left(\sigma ; \sigma^{\prime}\right)} \overline{\mathcal{M}}_{0, \rho}\right) \times \overline{\mathfrak{M}}_{\sigma ; B}\left(\mathbb{P}^{n}, d\right),
$$

where $\mathcal{A}_{0}\left(\sigma ; \sigma^{\prime}\right)=\left\{\rho=\left(I_{P} \sqcup J_{P}^{\prime},\left\{I_{k} \sqcup J_{k}: k \in K\right\}\right) \in \mathcal{A}_{0}\left([m], J_{P}\right):|K|+\left|I_{P}\right|=m^{\prime}\right\}$ 
and $\mathcal{A}_{0}\left([m], J_{P}\right)$ and $\overline{\mathcal{M}}_{0, \rho}$ are as in Section 2.2. Finally, if $\rho \in \mathcal{A}_{0}\left(\sigma ; \sigma^{\prime}\right)$ is as above,

$$
\begin{aligned}
\left.\iota_{\sigma}\right|_{\overline{\mathcal{M}}_{0, \rho} \times \overline{\mathfrak{M}}_{\sigma ; B}\left(\mathbb{P}^{n}, d\right)} T \overline{\mathfrak{M}}_{0, \sigma^{\prime}}\left(\mathbb{P}^{n}, d\right) / T\left(\overline{\mathcal{M}}_{0, \rho} \times \overline{\mathfrak{M}}_{\sigma ; B}\left(\mathbb{P}^{n}, d\right)\right) & \\
& =\bigoplus_{i \in[m]-I_{P}} \pi_{P}^{*} L_{i} \otimes \pi_{B}^{*} \pi_{i}^{*} L_{0} .
\end{aligned}
$$

The first claim in the second sentence and the claim of the third sentence in Lemma 3.7 follow immediately from the definition of the topology on $\overline{\mathfrak{M}}_{0,\{0\} \sqcup J}\left(\mathbb{P}^{n}, d\right)$. The remaining claims are also restatements of standard facts in GW-theory; they all follow from the description of the tangent bundle of $\overline{\mathfrak{M}}_{0,\{0\} \sqcup J}\left(\mathbb{P}^{n}, d\right)$ in $[10,(27.6)]$.

We finish this subsection by describing a natural bundle section

$$
\mathcal{D}_{0} \in \Gamma\left(\overline{\mathfrak{M}}_{0,\{0\} \sqcup J}\left(\mathbb{P}^{n}, d\right), \operatorname{Hom}\left(L_{0} ; \operatorname{ev}_{0}^{*} T \mathbb{P}^{n}\right)\right)
$$

which plays a central role in the rest of the paper. An element $[b] \in \overline{\mathfrak{M}}_{0,\{0\} \sqcup J}\left(\mathbb{P}^{n}, d\right)$ consists of a prestable nodal curve $\Sigma$ with marked points and a map $u: \Sigma \longrightarrow \mathbb{P}^{n}$. One of the marked points is labeled by 0 . We denote it by $x_{0}(b)$. We define $\mathcal{D}_{0}$ by

$$
\left.\mathcal{D}_{0}\right|_{b}=\left.d u\right|_{x_{0}(b)}: T_{x_{0}(b)} \Sigma \longrightarrow T_{\mathrm{ev}_{0}(b)} \mathbb{P}^{n}
$$

If $\mathfrak{U} \longrightarrow \overline{\mathfrak{M}}_{0,\{0\} \sqcup J}\left(\mathbb{P}^{n}, d\right)$ is the universal curve and ev: $\mathfrak{U} \longrightarrow \mathbb{P}^{n}$ is the natural evaluation map, then $\left.\mathcal{D}_{0}\right|_{b}$ is simply the restriction of $\left.d \mathrm{ev}\right|_{x_{0}(b)}$ to the vertical tangent bundle of $\mathfrak{U}$. The bundle section $\mathcal{D}_{0}$ vanishes identically along the subvarieties $\overline{\mathfrak{M}}_{0, \sigma}\left(\mathbb{P}^{n}, d\right)$ with $\sigma \in \mathcal{A}_{0}(d, J)$.

Lemma 3.8 If $d \in \mathbb{Z}^{+}$and $J$ is a finite set, the section $\mathcal{D}_{0}$ is transverse to the zero set on the complement of the subvarieties $\overline{\mathfrak{M}}_{0, \sigma}\left(\mathbb{P}^{n}, d\right)$ with $\sigma \in \mathcal{A}_{0}(d, J)$. Furthermore, for every

$$
\sigma \equiv\left(m ; J_{P}, J_{B}\right) \in \mathcal{A}_{0}(d, J)
$$

the differential of $\mathcal{D}_{0}$,

$$
\nabla \mathcal{D}_{0}: \mathcal{N}_{\iota_{\sigma}} \longrightarrow \iota_{\sigma}^{*} \operatorname{Hom}\left(L_{0}, \mathrm{ev}_{0}^{*} T \mathbb{P}^{n}\right)=\pi_{P}^{*} L_{0}^{*} \otimes \pi_{B}^{*} \mathrm{ev}_{0}^{*} T \mathbb{P}^{n},
$$

in the normal direction to the immersion $\iota_{\sigma}$ is given by

$$
\left.\nabla \mathcal{D}_{0}\right|_{\pi_{P}^{*} L_{i} \otimes \pi_{B}^{*} \pi_{i}^{*} L_{0}}=\pi_{P}^{*} s_{i} \otimes \pi_{B}^{*} \pi_{i}^{*} \mathcal{D}_{0} \quad \forall i \in[m],
$$

where $s_{i}$ is the homomorphism defined in Section 2.2.

The first claim of the lemma is an immediate consequence of the fact that

$$
H^{1}\left(\Sigma ; u^{*} T \mathbb{P}^{n} \otimes \mathcal{O}(-2 z)\right)=\{0\}
$$


for every genus-zero stable map $(\Sigma, u)$ and a smooth point $z \in \Sigma$ such that the restriction of $u$ to the irreducible component of $\Sigma$ containing $z$ is not constant. The second statement of the lemma follows from [21, Theorem 2.8].

\subsection{Initial data}

If $\boldsymbol{\aleph}$ and $J$ are finite sets and $d$ is positive integer, let

$$
\begin{gathered}
\overline{\mathfrak{M}}_{0,(\boldsymbol{\aleph}, J)}\left(\mathbb{P}^{n}, d\right)=\left\{\left(b_{l}\right)_{l \in \boldsymbol{N}} \in \prod_{l \in \boldsymbol{N}} \overline{\mathfrak{M}}_{0,\{0\} \sqcup J_{l}}\left(\mathbb{P}^{n}, d_{l}\right): d_{l} \in \mathbb{Z}^{+}, \sum_{l \in \boldsymbol{N}} d_{l}=d ;\right. \\
\left.\bigsqcup_{l \in \boldsymbol{N}} J_{l}=J ; \operatorname{ev}_{0}\left(b_{l}\right)=\operatorname{ev}_{0}\left(b_{l^{\prime}}\right) \forall l, l^{\prime} \in \boldsymbol{\aleph}\right\} ; \\
\mathfrak{M}_{0,(\boldsymbol{\aleph}, J)}\left(\mathbb{P}^{n}, d\right)=\left\{\left(b_{l}\right)_{l \in \boldsymbol{N}} \in \prod_{l \in \mathbb{N}} \mathfrak{M}_{0,\{0\} \sqcup J_{l}}\left(\mathbb{P}^{n}, d_{l}\right): d_{l} \in \mathbb{Z}^{+}, \sum_{l \in \boldsymbol{N}} d_{l}=d ;\right. \\
\left.\bigsqcup_{l \in \mathbb{N}} J_{l}=J ; \operatorname{ev}_{0}\left(b_{l}\right)=\operatorname{ev}_{0}\left(b_{l^{\prime}}\right) \forall l, l^{\prime} \in \boldsymbol{\aleph}\right\},
\end{gathered}
$$

where $\mathfrak{M}_{0,\{0\} \sqcup J_{l}}\left(\mathbb{P}^{n}, d_{l}\right)$ is the subset of $\overline{\mathfrak{M}}_{0,\{0\} \sqcup J_{l}}\left(\mathbb{P}^{n}, d_{l}\right)$ consisting of stable maps with smooth domains. For each $l \in \boldsymbol{\aleph}$, let

$$
\pi_{l}: \overline{\mathfrak{M}}_{0,(\aleph, J)}\left(\mathbb{P}^{n}, d\right) \longrightarrow \bigsqcup_{d_{l}>0, J_{l} \subset J} \overline{\mathfrak{M}}_{0,\{0\} \sqcup J_{l}}\left(\mathbb{P}^{n}, d_{l}\right)
$$

be the projection map. We put

$$
F_{(\aleph, J)}=\bigoplus_{l \in \aleph} \pi_{l}^{*} L_{0}
$$

where $L_{0} \longrightarrow \overline{\mathfrak{M}}_{0,\{0\} \sqcup J_{l}}\left(\mathbb{P}^{n}, d_{l}\right)$ is the universal tangent line bundle for the marked point 0 . In the next subsection, we construct a blowup

$$
\pi_{0,(\aleph, J)}: \widetilde{\mathfrak{M}}_{0,(\aleph, J)}\left(\mathbb{P}^{n}, d\right) \longrightarrow \mathbb{P} F_{(\aleph, J)}
$$

of the projective bundle $\mathbb{P} F_{(\aleph, J)}$ over $\overline{\mathfrak{M}}_{0,(\aleph, J)}\left(\mathbb{P}^{n}, d\right)$ and a line bundle

$$
\widetilde{\mathbb{E}} \longrightarrow \widetilde{\mathfrak{M}}_{0,(\aleph, J)}\left(\mathbb{P}^{n}, d\right) \text {. }
$$

We also describe a natural bundle section

$$
\widetilde{\mathcal{D}}_{(\aleph, J)} \in \Gamma\left(\widetilde{\mathfrak{M}}_{0,(\aleph, J)}\left(\mathbb{P}^{n}, d\right) ; \widetilde{\mathbb{E}}^{*} \otimes \pi_{0,(\aleph, J)}^{*} \pi_{\mathbb{P} F_{(\aleph, J)}}^{*} \operatorname{ev}_{0}^{*} T \mathbb{P}^{n}\right),
$$

where

$$
\pi_{\mathbb{P} F_{(\aleph, J)}}: \mathbb{P} F_{(\aleph, J)} \longrightarrow \overline{\mathfrak{M}}_{0,(\aleph, J)}\left(\mathbb{P}^{n}, d\right)
$$

is the bundle projection map. This section is transverse to the zero set. 
Similarly to Section 2.4 , the smooth variety $\widetilde{\mathfrak{M}}_{0,(\aleph, J)}\left(\mathbb{P}^{n}, d\right)$ is obtained by blowing up the subvarieties $\widetilde{\mathfrak{M}}_{0, \varrho}^{0}\left(\mathbb{P}^{n}, d\right)$ defined below and their proper transforms in an order consistent with a natural partial ordering $\prec$. The line bundle $\widetilde{\mathbb{E}}$ is the sum of the tautological line bundle

$$
\gamma_{(\aleph, J)} \longrightarrow \mathbb{P} F_{(\aleph, J)}
$$

and all exceptional divisors. The section $\widetilde{\mathcal{D}}_{(\aleph, J)}$ is induced from the sections $\pi_{l}^{*} \mathcal{D}_{0}$, with $l \in \boldsymbol{N}$, where $\mathcal{D}_{0}$ is as in Section 3.2.

If $\aleph, J$, and $d$ are as above, let

$$
\begin{array}{r}
\mathcal{A}_{0}(\boldsymbol{\aleph} ; d, J)=\left\{\left(\left(\sigma_{l}\right)_{l \in \mathbb{N}}, J_{B}\right):\left(\sigma_{l}, \varnothing\right) \in\{(0, \varnothing)\} \sqcup \mathcal{A}_{0}\left(d_{l}, J_{l, P}\right),\left(\sigma_{l}\right)_{l \in \mathbb{N}} \neq(0)_{l \in \mathbb{N}} ;\right. \\
\left.\sum_{l \in \mathbb{N}} d_{l}=d, J=J_{B} \sqcup \bigsqcup_{l \in \mathbb{N}} J_{l, P}\right\} .
\end{array}
$$

We define a partial ordering $\prec$ on $\mathcal{A}_{0}(\boldsymbol{\aleph} ; d, J)$ by setting

$$
\varrho^{\prime} \equiv\left(\left(\sigma_{l}^{\prime}\right)_{l \in \mathbb{N}}, J_{B}^{\prime}\right) \prec \varrho \equiv\left(\left(\sigma_{l}\right)_{l \in \mathbb{N}}, J_{B}\right)
$$

if $\varrho^{\prime} \neq \varrho$ and for every $l \in \boldsymbol{\aleph}$ either $\sigma_{l}^{\prime}=\sigma_{l},\left(\sigma_{l}^{\prime}, \varnothing\right) \prec\left(\sigma_{l}, \varnothing\right)$, or $\sigma_{l}^{\prime}=0$. If $\varrho \in \mathcal{A}_{0}(\boldsymbol{\aleph} ; d, J)$ is as in (3-5), we put

$$
\boldsymbol{\aleph}_{P}(\varrho)=\left\{l \in \boldsymbol{N}: \sigma_{l} \neq 0\right\} \quad \text { and } \quad \boldsymbol{\aleph}_{S}(\varrho)=\left\{l \in \boldsymbol{N}: \sigma_{l}=0\right\} .
$$

Here $P$ and $S$ stand for the subsets of principal and secondary elements of $\boldsymbol{\aleph}$, respectively; see the next paragraph. Note that

$$
\begin{aligned}
& \varrho^{\prime} \prec \varrho \Longrightarrow \aleph_{P}\left(\varrho^{\prime}\right) \subset \aleph_{P}(\varrho), \quad \aleph_{P}(\varrho) \neq \varnothing \forall \varrho \in \mathcal{A}_{0}(\boldsymbol{\aleph} ; d, J), \\
& \varrho=\left(\left(m_{l} ; J_{l, P}\right)_{l \in \boldsymbol{\aleph}_{P}(\varrho)},(0)_{l \in \boldsymbol{\aleph}_{S}(\varrho)}, J_{B}\right)
\end{aligned}
$$

for some $m_{l}$ and $J_{l, P}$. Choose an ordering $<$ on $\mathcal{A}_{0}(\boldsymbol{\aleph} ; d, J)$ extending the partial ordering $\prec$. We denote the corresponding minimal and maximal element by $\varrho_{\text {min }}$ and $\varrho_{\max }$, respectively. For every $\varrho \in \mathcal{A}_{0}(\aleph ; d, J)$, define

$$
\varrho-1 \in\{0\} \sqcup \mathcal{A}_{0}(\aleph ; d, J)
$$

as in (2-7).

If $\varrho \in \mathcal{A}_{0}(\aleph ; d, J)$ is as in (3-5), let

$$
\begin{array}{r}
\overline{\mathfrak{M}}_{0, \varrho}\left(\mathbb{P}^{n}, d\right)=\left\{\left(b_{l}\right)_{l \in \mathbb{N}} \in \prod_{l \in \mathbb{N}} \overline{\mathfrak{M}}_{0,\left(\sigma_{l}, J_{l, B}\right)}\left(\mathbb{P}^{n}, d_{l}\right): \sum_{l \in \mathbb{N}} d_{l}=d ; \bigsqcup_{l \in \mathbb{N}} J_{l, B}=J_{B} ;\right. \\
\left.\operatorname{ev}_{0}\left(b_{l_{1}}\right)=\operatorname{ev}_{0}\left(b_{l_{2}}\right) \forall l_{1}, l_{2} \in \boldsymbol{N}\right\} .
\end{array}
$$

Geometry 83 Topology, Volume 12 (2008) 
This is a subset of $\overline{\mathfrak{M}}_{0,(\aleph, J)}\left(\mathbb{P}^{n}, d\right)$. With $\bullet=P, S$, we define

$$
F_{\varrho ; \bullet}=\left.\left.\bigoplus_{l \in \aleph_{\bullet}(\varrho)} \pi_{l}^{*} L_{0}\right|_{\overline{\mathfrak{M}}_{0, \varrho}\left(\mathbb{P}^{n}, d\right)} \subset F_{(\boldsymbol{\aleph}, J)}\right|_{\overline{\mathfrak{M}}_{0, \varrho}\left(\mathbb{P}^{n}, d\right)} .
$$

Let

$$
\widetilde{\mathfrak{M}}_{0, \varrho}^{0}\left(\mathbb{P}^{n}, d\right)=\mathbb{P} F_{\varrho ; P} \subset \widetilde{\mathfrak{M}}_{0,(\aleph, J)}^{0}\left(\mathbb{P}^{n}, d\right) \equiv \mathbb{P} F_{(\aleph, J)}
$$

From Lemma 3.6, we immediately obtain

Lemma 3.9 If $\varrho_{1}, \varrho_{2} \in \mathcal{A}_{0}(\aleph ; d, J), \varrho_{1} \neq \varrho_{2} \varrho_{1} \nprec \varrho_{2}$, and $\varrho_{2} \nprec \varrho_{1}$, then

where

$$
\begin{gathered}
\widetilde{\mathfrak{M}}_{0, \varrho_{1}}^{0}\left(\mathbb{P}^{n}, d\right) \cap \widetilde{\mathfrak{M}}_{0, \varrho_{2}}^{0}\left(\mathbb{P}^{n}, d\right) \subset \widetilde{\mathfrak{M}}_{0, \widetilde{\varrho}\left(\varrho_{1}, \varrho_{2}\right)}^{0}\left(\mathbb{P}^{n}, d\right), \\
\widetilde{\varrho}\left(\varrho_{1}, \varrho_{2}\right)=\max \left\{\varrho^{\prime} \in \mathcal{A}_{0}(\boldsymbol{\aleph} ; d, J): \varrho^{\prime} \prec \varrho_{1}, \varrho_{2}\right\} .
\end{gathered}
$$

If $\widetilde{\varrho}\left(\varrho_{1}, \varrho_{2}\right)$ is not defined, $\widetilde{\mathfrak{M}}_{0, \varrho_{1}}^{0}\left(\mathbb{P}^{n}, d\right)$ and $\widetilde{\mathfrak{M}}_{0, \varrho_{2}}^{0}\left(\mathbb{P}^{n}, d\right)$ are disjoint.

With $\varrho$ as (3-6), let

$$
\varrho_{P}=\left(\left[m_{l}\right], J_{l, P}\right)_{l \in \aleph_{P}(\varrho)}, \aleph_{B}(\varrho)=\aleph_{S}(\varrho) \sqcup \bigsqcup_{l \in \aleph_{P}(\varrho)}\left[m_{l}\right], J_{B}(\varrho)=J_{B}, G_{\varrho}=\prod_{l \in \boldsymbol{\aleph}_{P}(\varrho)} S_{m_{l}} .
$$

With $\widetilde{\mathcal{M}}_{0, \varrho_{P}}^{0}$ as in Section 2.4, we denote by

$$
\iota_{0, \varrho}: \widetilde{\mathcal{M}}_{0, \varrho_{P}}^{0} \times \overline{\mathfrak{M}}_{0,\left(\aleph_{B}(\varrho), J_{B}(\varrho)\right)}\left(\mathbb{P}^{n}, d\right) \longrightarrow \widetilde{\mathfrak{M}}_{0, \varrho}^{0}\left(\mathbb{P}^{n}, d\right) \subset \widetilde{\mathfrak{M}}_{0,(\aleph, J)}^{0}\left(\mathbb{P}^{n}, d\right)
$$

the natural node-identifying map induced by the immersions $\iota_{\left(\sigma_{l}, J_{l, B}\right)}$ in (3-4). It descends to an immersion

$$
\bar{\iota}_{0, \varrho}:\left(\widetilde{\mathcal{M}}_{0, \varrho P}^{0} \times \overline{\mathfrak{M}}_{0,\left(\aleph_{B}(\varrho), J_{B}(\varrho)\right)}\left(\mathbb{P}^{n}, d\right)\right) / G_{\varrho} \longrightarrow \widetilde{\mathfrak{M}}_{0,(\aleph, J)}^{0}\left(\mathbb{P}^{n}, d\right) .
$$

Let

$$
\pi_{P}, \pi_{B}: \widetilde{\mathcal{M}}_{0, \varrho_{P}}^{0} \times \overline{\mathfrak{M}}_{0,\left(\aleph_{B}(\varrho), J_{B}(\varrho)\right)}\left(\mathbb{P}^{n}, d\right) \longrightarrow \widetilde{\mathcal{M}}_{0, \varrho_{P}}^{0}, \overline{\mathfrak{M}}_{0,\left(\aleph_{B}(\varrho), J_{B}(\varrho)\right)}\left(\mathbb{P}^{n}, d\right)
$$

be the projection maps.

For the rest of this section, as well as for Section 4, we take

$$
\begin{gathered}
\overline{\mathfrak{M}}_{0,(\aleph, J)}=\overline{\mathfrak{M}}_{0,(\aleph, J)}\left(\mathbb{P}^{n}, d\right), \mathfrak{M}_{0,(\aleph, J)}=\mathfrak{M}_{0,(\aleph, J)}\left(\mathbb{P}^{n}, d\right), \\
\widetilde{\mathfrak{M}}_{0,(\aleph, J)}^{0}=\widetilde{\mathfrak{M}}_{0,(\aleph, J)}^{0}\left(\mathbb{P}^{n}, d\right), \widetilde{\mathfrak{M}}_{0, \varrho}^{0}=\widetilde{\mathfrak{M}}_{0, \varrho}^{0}\left(\mathbb{P}^{n}, d\right) \quad \forall \varrho \in \mathcal{A}_{0}(\aleph ; d, J),
\end{gathered}
$$

for any pair $(\boldsymbol{\aleph}, J)$ as above. 
Lemma 3.10 If $\aleph$ and $J$ are finite sets and $d \in \mathbb{Z}^{+}$, the collections

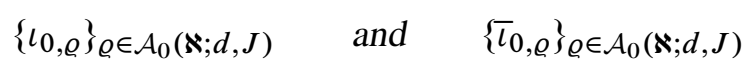

of immersions are properly self-intersecting. If

$$
\varrho^{*} \equiv\left(\left(m_{l}^{*} ; J_{l, P}^{*}\right)_{l \in \boldsymbol{\aleph}_{P}\left(\varrho^{*}\right)},(0)_{l \in \boldsymbol{\aleph}_{S}\left(\varrho^{*}\right)}, J_{B}^{*}\right) \in \mathcal{A}_{0}(\boldsymbol{\aleph} ; d, J),
$$

then

$$
\operatorname{Im}^{s} \bar{\iota}_{0, \varrho^{*}} \subset \bigcup_{\varrho^{\prime} \prec \varrho^{*}} \widetilde{\mathfrak{M}}_{0, \varrho^{\prime}}^{0},
$$

$$
\mathcal{N}_{l_{0, \varrho^{*}}}=\bigoplus_{l \in \mathbb{N}_{P}\left(\varrho^{*}\right)} \bigoplus_{i \in\left[m_{l}^{*}\right]} \pi_{P}^{*} L_{0,(l, i)} \otimes \pi_{B}^{*} \pi_{(l, i)}^{*} L_{0} \oplus \pi_{P}^{*} \mathbb{E}_{0}^{*} \otimes \pi_{B}^{*} \bigoplus_{l \in \mathbb{N}_{S}\left(\varrho^{*}\right)} \pi_{l}^{*} L_{0}
$$

where $\mathbb{E}_{0}$ and $L_{0,(l, i)}$ are as in Sections 2.4. If $\varrho, \varrho^{*} \in \mathcal{A}_{0}(\aleph ; d, J), \varrho$ is as (3-6), and $\varrho \prec \varrho^{*}$, then

$$
\iota_{0, \varrho^{*}}^{-1}\left(\widetilde{\mathfrak{M}}_{0, \varrho}^{0}\right)=\left(\underset{\rho \in \mathcal{A}_{0}\left(\varrho^{*} ; \varrho\right)}{\bigcup} \widetilde{\mathcal{M}}_{0, \rho}^{0}\right) \times \overline{\mathfrak{M}}_{0,\left(\aleph_{B}\left(\varrho^{*}\right), J_{B}\left(\varrho^{*}\right)\right)}
$$

where $\mathcal{A}_{0}\left(\varrho^{*} ; \varrho\right)$

$$
\begin{array}{r}
=\left\{\rho=\left(\aleph_{P}(\varrho),\left(I_{l, P}^{*} \sqcup J_{l, P},\left\{I_{l, k}^{*} \sqcup J_{l, k}^{*}: k \in K_{l}^{*}\right\}\right)_{l \in \boldsymbol{\aleph}_{P}\left(\varrho^{*}\right)}\right) \in \mathcal{A}_{0}\left(\varrho_{P}^{*}\right):\right. \\
\left.\left|K_{l}^{*}\right|+\left|I_{l, P}^{*}\right|=m_{l} \forall l \in \aleph_{P}\left(\varrho^{*}\right)\right\}
\end{array}
$$

and $\mathcal{A}_{0}\left(\varrho_{P}^{*}\right)$ and $\widetilde{\mathcal{M}}_{0, \rho}^{0}$ are as in Section 2.4. Finally, if $\rho \in \mathcal{A}_{0}\left(\varrho^{*} ; \varrho\right)$ is as above,

$$
\begin{gathered}
\left.\iota_{0, \varrho^{*}}\right|_{\widetilde{\mathcal{M}}_{0, \rho}^{0} \times \overline{\mathfrak{M}}_{0,\left(\aleph_{B}\left(\varrho^{*}\right), J_{B}\left(\varrho^{*}\right)\right)} T \overline{\mathfrak{M}}_{0, \varrho} / T\left(\widetilde{\mathcal{M}}_{0, \rho}^{0} \times \overline{\mathfrak{M}}_{0,\left(\aleph_{B}\left(\varrho^{*}\right), J_{B}\left(\varrho^{*}\right)\right)}\right)} ^{=} \bigoplus_{l \in \boldsymbol{\aleph}_{P}\left(\varrho^{*}\right)-\aleph_{P}(\varrho)} \bigoplus_{i \in\left[m_{l}^{*}\right]} \pi_{P}^{*} L_{0,(l, i)} \otimes \pi_{B}^{*} \pi_{(l, i)}^{*} L_{0} \\
\oplus \bigoplus_{l \in \aleph_{P}(\varrho)} \bigoplus_{i \in\left[m_{l}^{*}\right]-I_{l, P}^{*}} \pi_{P}^{*} L_{0,(l, i)} \otimes \pi_{B}^{*} \pi_{(l, i)}^{*} L_{0} .
\end{gathered}
$$

The normal bundle $\mathcal{N}_{\iota_{0, \varrho^{*}}}$ for the immersion $\iota_{0, \varrho^{*}}$ splits into horizontal and vertical bundles:

$$
\mathcal{N}_{\iota_{0, \varrho^{*}}}=\mathcal{N}_{\iota_{0, e^{*}}}^{\perp} \oplus \mathcal{N}_{\iota_{0, \ell^{*}}}^{\top} .
$$

It is immediate from the definitions that

$$
\mathcal{N}_{\iota_{0, \varrho^{*}}}^{\top}=\iota_{0, \varrho^{*}}^{*}\left(\gamma_{(\aleph, J)}^{*} \otimes F_{\varrho ; S}\right)=\pi_{P}^{*} \mathbb{E}_{0}^{*} \otimes \pi_{B}^{*} \underset{l \in \aleph_{S}\left(\varrho^{*}\right)}{\bigoplus} \pi_{l}^{*} L_{0}
$$


The horizontal normal bundle $\mathcal{N}_{\iota_{0, e^{*}}}^{\perp}$ is the pullback of the normal bundle for the node identifying immersion

$$
\overline{\mathcal{M}}_{0, \varrho_{P}^{*}} \times \overline{\mathfrak{M}}_{0,\left(\aleph_{B}\left(\varrho^{*}\right), J_{B}\left(\varrho^{*}\right)\right)} \longrightarrow \overline{\mathfrak{M}}_{0, \varrho^{*}}\left(\mathbb{P}^{n}, d\right) \subset \overline{\mathfrak{M}}_{0,(\aleph, J)}
$$

induced by the immersions $\iota_{\left(\sigma_{l}^{*}, J_{l, B}^{*}\right)}$ in (3-4) by the bundle projection map $\pi_{\mathbb{P} F_{Q ; P}}$. The normal bundle for this immersion is the sum of component-wise normal bundles given by Lemma 3.7. The remaining claims of Lemma 3.10 follow easily from the corresponding statements of Lemma 3.7 as well.

We note that for every $\varrho^{*} \in \mathcal{A}_{0}(\aleph ; d, J)$,

$$
\mathcal{A}_{0}\left(\varrho_{P}^{*}\right)=\bigsqcup_{\varrho \prec \varrho^{*}} \mathcal{A}_{0}\left(\varrho^{*} ; \varrho\right)
$$

Furthermore, if $\varrho_{1}, \varrho_{2} \in \mathcal{A}_{0}(\aleph ; d, J)$ are such that $\varrho_{1}, \varrho_{2} \prec \varrho^{*}$, then

$$
\rho_{1}^{*} \in \mathcal{A}_{0}\left(\varrho^{*} ; \varrho_{1}\right), \quad \rho_{2}^{*} \in \mathcal{A}_{0}\left(\varrho^{*} ; \varrho_{2}\right), \quad \rho_{1}^{*} \prec \rho_{2}^{*} \quad \Longrightarrow \quad \varrho_{1} \prec \varrho_{2}
$$

Thus, we can choose an ordering $<$ on $\mathcal{A}_{0}\left(\varrho_{P}^{*}\right)$ extending the partial ordering $\prec$ of Section 2.4 such that

$$
\varrho_{1}<\varrho_{2}, \quad \rho_{1}^{*} \in \mathcal{A}_{0}\left(\varrho^{*} ; \varrho_{1}\right), \quad \rho_{2}^{*} \in \mathcal{A}_{0}\left(\varrho^{*} ; \varrho_{2}\right) \quad \Longrightarrow \quad \rho_{1}^{*}<\rho_{2}^{*},
$$

whenever $\varrho_{1}, \varrho_{2} \in \mathcal{A}_{0}(\aleph ; d, J)$ are such that $\varrho_{1}, \varrho_{2} \prec \varrho^{*}$. In the next subsection, we will refer to the blowup construction of Section 2.4 corresponding to such an ordering.

Via the projection maps $\pi_{l}$, the bundle sections $\mathcal{D}_{0}$ of Section 3.2 induce a linear bundle map

$$
\mathcal{D}_{(\aleph, J)}: F_{(\aleph, J)} \longrightarrow \mathrm{ev}_{0}^{*} T \mathbb{P}^{n}
$$

over $\overline{\mathfrak{M}}_{0,(\aleph, J)}$. In turn, this homomorphism induces a bundle section

$$
\widetilde{\mathcal{D}}_{0} \in \Gamma\left(\widetilde{\mathfrak{M}}_{0,(\aleph, J)}^{0} ; \mathbb{E}_{0}^{*} \otimes \pi_{\mathbb{P} F_{(\aleph, J)}}^{*} \mathrm{ev}_{0}^{*} T \mathbb{P}^{n}\right), \quad \text { where } \quad \mathbb{E}_{0}=\gamma_{(\aleph, J)} \longrightarrow \widetilde{\mathfrak{M}}_{0,(\aleph, J)}^{0}
$$

This section vanishes identically on the subvarieties $\widetilde{\mathfrak{M}}_{0, \varrho}^{0}$ of $\widetilde{\mathfrak{M}}_{0,(\aleph, J)}^{0}$ with $\varrho \in$ $\mathcal{A}_{0}^{*}(\aleph ; d, J)$.

Lemma 3.11 The section $\widetilde{\mathcal{D}}_{0}$ is transverse to the zero set on the complement of the subvarieties $\widetilde{\mathfrak{M}}_{0, \varrho^{*}}^{0}$ with $\varrho^{*} \in \mathcal{A}_{0}(\boldsymbol{\aleph} ; d, J)$. Furthermore, for every $\varrho^{*} \in \mathcal{A}_{0}(\aleph ; d, J)$ as in Lemma 3.10, the differential of $\widetilde{\mathcal{D}}_{0}$,

$$
\nabla \widetilde{\mathcal{D}}_{0}: \mathcal{N}_{\iota_{0, \varrho^{*}}} \longrightarrow \iota_{0, \varrho^{*}}^{*}\left(\mathbb{E}_{0}^{*} \otimes \pi_{\mathbb{P} F_{(\aleph, J)}^{*}} e_{0}^{*} T \mathbb{P}^{n}\right)=\pi_{P}^{*} \mathbb{E}_{0}^{*} \otimes \pi_{B}^{*} \operatorname{ev}_{0}^{*} T \mathbb{P}^{n},
$$


in the normal direction to the immersion $\iota_{0, \varrho^{*}}$ is given by

$$
\begin{aligned}
&\left.\nabla \widetilde{\mathcal{D}}_{0}\right|_{\pi_{P}^{*} L_{0,(l, i)} \otimes \pi_{B}^{*} \pi_{(l, i)}^{*} L_{0}}=\pi_{P}^{*} s_{0,(l, i)} \otimes \pi_{B}^{*} \pi_{(l, i)}^{*} \mathcal{D}_{0} \forall i \in\left[m_{l}^{*}\right], l \in \aleph_{P}\left(\varrho^{*}\right), \\
&\left.\nabla \widetilde{\mathcal{D}}_{0}\right|_{\mathcal{N}_{\iota_{0, \varrho^{*}}^{\top}}^{\top}}=\pi_{P}^{*} \mathrm{id} \otimes \pi_{B}^{*} \mathcal{D}_{\left(\aleph_{B}\left(\varrho^{*}\right), J_{B}\left(\varrho^{*}\right)\right),}
\end{aligned}
$$

where $s_{0,(l, i)}$ is the homomorphism defined in Section 2.4.

This lemma follows immediately from Lemma 3.8.

\subsection{Inductive construction}

We are now ready to describe the inductive assumptions for our construction of the blowup

$$
\pi_{0,(\aleph, J)}: \widetilde{\mathfrak{M}}_{0,(\aleph, J)}^{\varrho_{\max }} \equiv \widetilde{\mathfrak{M}}_{0,(\aleph, J)}\left(\mathbb{P}^{n}, d\right) \longrightarrow \widetilde{\mathfrak{M}}_{0,(\aleph, J)}^{0} \equiv \widetilde{\mathfrak{M}}_{0,(\aleph, J)}^{0}\left(\mathbb{P}^{n}, d\right) .
$$

Suppose $\varrho \in \mathcal{A}_{0}(\boldsymbol{\aleph} ; d, J)$ and we have constructed

(I1) a blowup

$$
\pi_{\varrho-1}: \widetilde{\mathfrak{M}}_{0,(\aleph, J)}^{\varrho-1} \longrightarrow \widetilde{\mathfrak{M}}_{0,(\aleph, J)}^{0}
$$

such that $\pi_{\varrho-1}$ is an isomorphism outside of the preimage of the spaces $\widetilde{\mathfrak{M}}_{0, \varrho^{\prime}}^{0}$ with $\varrho^{\prime} \leq \varrho-1$;

(I2) a line bundle $\mathbb{E}_{\varrho-1} \longrightarrow \widetilde{\mathfrak{M}}_{0,(\aleph, J)}^{\varrho-1}$;

(I3) a section $\widetilde{\mathcal{D}}_{\varrho-1} \in \Gamma\left(\widetilde{\mathfrak{M}}_{0,(\aleph, J)}^{\varrho-1} ; \mathbb{E}_{\varrho-1}^{*} \otimes \pi_{\varrho-1}^{*} \pi_{\mathbb{P} F_{(\aleph, J)}}^{*} \mathrm{ev}_{0}^{*} T \mathbb{P}^{n}\right)$.

For each $\varrho^{*}>\varrho-1$, let

$$
\widetilde{\mathfrak{M}}_{0, \varrho^{*}}^{\varrho-1} \equiv \widetilde{\mathfrak{M}}_{0, \varrho^{*}}^{\varrho-1}\left(\mathbb{P}^{n}, d\right) \subset \widetilde{\mathfrak{M}}_{0,(\aleph, J)}^{\varrho-1}
$$

be the proper transform of $\widetilde{\mathfrak{M}}_{0, \varrho^{*}}^{0}$ in $\widetilde{\mathfrak{M}}_{0,(\aleph, J)}^{\varrho-1}$. We assume that

(I4) the section $\widetilde{\mathcal{D}}_{\varrho-1}$ is transverse to the zero set on the complement of the subvarieties $\widetilde{\mathfrak{M}}_{0, \varrho^{*}}^{\varrho-1}$ with $\varrho^{*}>\varrho-1$ and vanishes identically along these subvarieties;

(I5) if $\varrho_{1}, \varrho_{2} \in \mathcal{A}_{0}(\aleph ; d, J)$ are such that $\varrho_{1} \neq \varrho_{2}, \varrho_{1} \nprec \varrho_{2}, \varrho_{2} \nprec \varrho_{1}$, and $\varrho-1<\varrho_{1}, \varrho_{2}$, then

$$
\widetilde{\mathfrak{M}}_{0, \varrho_{1}}^{\varrho-1} \cap \widetilde{\mathfrak{M}}_{0, \varrho_{2}}^{\varrho-1} \begin{cases}\subset \widetilde{\mathfrak{M}}_{0, \widetilde{\varrho}\left(\varrho_{1}, \varrho_{2}\right)}^{\varrho-1}, & \text { if } \widetilde{\varrho}\left(\varrho_{1}, \varrho_{2}\right)>\varrho-1 ; \\ =\varnothing, & \text { otherwise, }\end{cases}
$$

where $\widetilde{\varrho}\left(\varrho_{1}, \varrho_{2}\right)$ is as in Lemma 3.10 . 
We also assume that for all $\varrho^{*} \in \mathcal{A}_{0}(\boldsymbol{\aleph} ; d, J)$ such that $\varrho^{*}>\varrho-1$ :

(I6) the domain of the $G_{\varrho^{*}}$-invariant immersion $\iota_{\varrho-1, \varrho^{*}}$ induced by $\iota_{0, \varrho^{*}}$ is

$$
\widetilde{\mathcal{M}}_{0, \varrho_{P}^{*}}^{\rho_{Q^{*}}(\varrho-1)} \times \overline{\mathfrak{M}}_{0,\left(\aleph_{B}\left(\varrho^{*}\right), J_{B}\left(\varrho^{*}\right)\right)},
$$

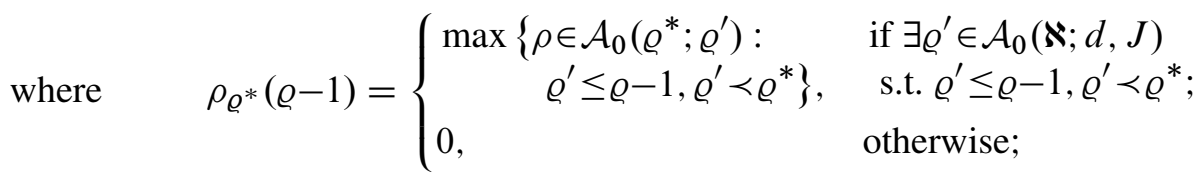

(I7) if $\varrho^{\prime} \in \mathcal{A}_{0}(\aleph ; d, J)$ is such that $\varrho-1<\varrho^{\prime} \prec \varrho^{*}$, then

$$
\iota_{\varrho-1, \varrho^{*}}^{-1}\left(\widetilde{\mathfrak{M}}_{0, \varrho^{\prime}}^{\varrho-1}\right)=\left(\underset{\rho \in \mathcal{A}_{0}\left(\varrho^{*} ; \varrho^{\prime}\right)}{\bigcup} \widetilde{\mathcal{M}}_{0, \rho}^{\rho_{\varrho^{*}}(\varrho-1)}\right) \times \overline{\mathfrak{M}}_{0,\left(\aleph_{B}\left(\varrho^{*}\right), J_{B}\left(\varrho^{*}\right)\right)} ;
$$

(I8) $\operatorname{Im}^{s} \bar{\iota}_{\varrho-1, \varrho^{*}} \subset \bigcup_{\varrho-1<\varrho^{\prime} \prec \varrho^{*}} \widetilde{\mathfrak{M}}_{0, \varrho^{\prime}}^{\varrho-1}$, where

$$
\bar{\iota}_{\varrho-1, \varrho^{*}}:\left(\widetilde{\mathcal{M}}_{0, \varrho_{P}^{*}}^{\rho_{Q^{*}}(\varrho-1)} \times \overline{\mathfrak{M}}_{0,\left(\aleph_{B}\left(\varrho^{*}\right), J_{B}\left(\varrho^{*}\right)\right)}\right) / G_{\varrho^{*}} \longrightarrow \widetilde{\mathfrak{M}}_{0,(\aleph, J)}^{\varrho-1},
$$

is the immersion map induced by $\iota_{\varrho-1, \varrho^{*}}$.

Furthermore, we assume that

(I9) the collections of immersions

$$
\left\{\iota_{\varrho-1, \varrho^{*}}\right\}_{\varrho^{*} \in \mathcal{A}_{0}(\boldsymbol{\aleph} ; d, J), \varrho^{*}>\varrho-1} \quad \text { and } \quad\left\{\bar{\iota}_{\varrho}-1, \varrho^{*}\right\}_{\varrho}-1 \in \mathcal{A}_{0}(\boldsymbol{\aleph} ; d, J), \varrho^{*}>\varrho-1
$$

are properly self-intersecting.

Finally, for all $\varrho^{*} \in \mathcal{A}_{0}(\aleph ; d, J)$ such that $\varrho^{*}>\varrho-1$ :

(I10) $\iota_{\varrho-1, \varrho^{*}}^{*} \mathbb{E}_{\varrho-1}=\pi_{P}^{*} \mathbb{E}_{\rho_{\varrho^{*}}(\varrho-1)}$, where

$$
\pi_{P}, \pi_{B}: \widetilde{\mathcal{M}}_{0, \varrho_{P}^{*}}^{\rho_{\varrho^{*}}(\varrho-1)} \times \overline{\mathfrak{M}}_{0,\left(\aleph_{B}\left(\varrho^{*}\right), J_{B}\left(\varrho^{*}\right)\right)} \longrightarrow \widetilde{\mathcal{M}}_{0, \varrho_{P}^{*}}^{\rho_{\varrho^{*}}(\varrho-1)}, \overline{\mathfrak{M}}_{0,\left(\aleph_{B}\left(\varrho^{*}\right), J_{B}\left(\varrho^{*}\right)\right)}
$$

are the two projection maps;

(I11) if $\varrho^{*}$ is as in Lemma 3.10, then the normal bundle for the immersion $\iota_{\varrho-1, \varrho^{*}}$ is given by

$$
\begin{aligned}
\mathcal{N}_{l_{Q-1, Q^{*}}} & =\mathcal{N}_{l_{Q-1, Q^{*}}}^{\perp} \oplus \mathcal{N}_{l_{Q-1, Q^{*}}^{\top}}^{\top} \bigoplus_{l \in \mathcal{N}_{P}\left(\varrho^{*}\right)} \bigoplus_{i \in\left[m_{l}^{*}\right]} \pi_{P}^{*} L_{\rho_{Q^{*}}(\varrho-1),(l, i)} \otimes \pi_{B}^{*} \pi_{(l, i)}^{*} L_{0} \\
& \oplus \pi_{P}^{*} \mathbb{E}_{\rho_{Q^{*}}^{*}(\varrho-1)} \otimes \pi_{B}^{*} \bigoplus_{l \in \boldsymbol{N}_{S}\left(\varrho^{*}\right)} \pi_{l}^{*} L_{0},
\end{aligned}
$$


where $L_{\rho_{\varrho^{*}}(\varrho-1),(l, i)}, \mathbb{E}_{\rho_{\varrho^{*}}(\varrho-1)} \longrightarrow \widetilde{\mathcal{M}}_{0, \varrho_{P}^{*}}^{\rho_{\varrho^{*}}(\varrho-1)}$ are the line bundles constructed in Section 2.4;

(I12) the differential of $\widetilde{\mathcal{D}}_{\varrho-1}$,

$$
\begin{aligned}
\nabla \widetilde{\mathcal{D}}_{\varrho-1}: \mathcal{N}_{\iota_{Q-1, \varrho^{*}}} \longrightarrow \iota_{\varrho-1, \varrho^{*}}^{*}\left(\mathbb{E}_{\varrho-1}^{*} \otimes \pi_{\varrho-1}^{*} \pi_{\mathbb{P} F_{(\aleph, J)}}^{*} \mathrm{ev}_{0}^{*} T \mathbb{P}^{n}\right) \\
=\pi_{P}^{*} \mathbb{E}_{\rho_{\varrho^{*}}^{*}(\varrho-1)}^{*} \otimes \pi_{B}^{*} \operatorname{ev}_{0}^{*} T \mathbb{P}^{n},
\end{aligned}
$$

in the normal direction to the immersion $\iota_{\varrho-1, \varrho^{*}}$ is given by

$$
\left.\nabla \widetilde{\mathcal{D}}_{\varrho-1}\right|_{\pi_{P}^{*} L_{\rho_{\varrho^{*}(\varrho-1),(l, i)}} \otimes \pi_{B}^{*} \pi_{(l, i)}^{*} L_{0}}=\pi_{P}^{*} s_{\rho_{\varrho^{*}}(\varrho-1),(l, i)} \otimes \pi_{B}^{*} \pi_{(l, i)}^{*} \mathcal{D}_{0}
$$

for all $i \in\left[m_{l}^{*}\right], l \in \aleph_{P}\left(\varrho^{*}\right)$ and

$$
\left.\nabla \widetilde{\mathcal{D}}_{\varrho-1}\right|_{\mathcal{N}_{\varrho^{-}-1, \varrho^{*}}^{\top}}=\pi_{P}^{*} \mathrm{id} \otimes \pi_{B}^{*} \mathcal{D}_{\left(\aleph_{B}\left(\varrho^{*}\right), J_{B}\left(\varrho^{*}\right)\right)},
$$

where $s_{\rho_{Q^{*}}(\varrho-1),(l, i)}$ is the homomorphism defined in Section 2.4.

By the inductive assumption ( I4), the loci on which the sections $\widetilde{\mathcal{D}}_{\varrho}$ fail to be transverse to the zero set shrink and eventually disappear. For each $\varrho$, the behavior of $\widetilde{\mathcal{D}}_{\varrho}$ in the directions normal to the "bad" locus is described by (I12). By the inductive assumption (I5), if $\varrho_{1}$ and $\varrho_{2}$ are noncomparable elements of $\left(\mathcal{A}_{0}(\boldsymbol{\aleph} ; d, J), \prec\right)$, the proper transforms of $\widetilde{\mathfrak{M}}_{0, \varrho_{1}}^{0}$ and $\widetilde{\mathfrak{M}}_{0, \varrho_{2}}^{0}$ become disjoint by the time either is ready to be blown up for any ordering $<$ extending the partial ordering $\prec$. Similarly to Sections 2.3 and 2.4, (I5) will imply that the end result of the present blowup construction is independent of the choice of an extension $<$. By (I6), our blowup construction modifies each immersion $\iota_{0, e^{*}}$ by changing the first factor of the domain according to the blowup construction of Section 2.4, until a proper transform of the image of $\iota_{0, \varrho^{*}}$ is to be blown up; see below. By ( $\left.I 8\right)$, by the time this happens the immersion $\bar{\iota}_{0, \varrho^{*}}$ induced by $\iota_{0, \varrho^{*}}$ transforms into an embedding. Thus, all blowup loci are smooth.

We note that all of the assumptions $(I 1)-(I 12)$ are satisfied if $\varrho-1$ is replaced by 0 . In particular, (I5) is a restatement of Lemma 3.9, while (I4) and (I12) are the two parts of Lemma 3.11. The statements (I7)-(I11), with $\varrho-1$ replaced by 0 , are contained in Lemma 3.10.

If $\varrho \in \mathcal{A}_{0}(\aleph ; d, J)$ is as above, let

$$
\tilde{\pi}_{\varrho}: \widetilde{\mathfrak{M}}_{0,(\aleph, J)}^{\varrho} \longrightarrow \widetilde{\mathfrak{M}}_{0,(\aleph, J)}^{\varrho-1}
$$

be the blowup of $\widetilde{\mathfrak{M}}_{0,(\aleph, J)}^{\varrho-1}$ along $\widetilde{\mathfrak{M}}_{0, \varrho}^{\varrho-1}$, which is a smooth subvariety by the inductive assumption (I8). We denote the exceptional divisor for this blowup by $\widetilde{\mathfrak{M}}_{0, \varrho}^{\varrho}$. If $\varrho^{*}>\varrho$, 
let $\widetilde{\mathfrak{M}}_{0, \varrho^{*}}^{\varrho} \subset \widetilde{\mathfrak{M}}_{0,(\aleph, J)}^{\varrho}$ be the proper transform of $\widetilde{\mathfrak{M}}_{0, \varrho^{*}}^{\varrho-1}$. We put

$$
\mathbb{E}_{\varrho}=\tilde{\pi}_{\varrho}^{*} \mathbb{E}_{\varrho-1} \otimes \mathcal{O}\left(\widetilde{\mathfrak{M}}_{0, \varrho}^{\varrho}\right) .
$$

The section $\widetilde{\pi}_{\varrho}^{*} \widetilde{\mathcal{D}}_{\varrho-1}$ vanishes identically along the divisor $\widetilde{\mathfrak{M}}_{0, \varrho}^{\varrho}$. Thus, it induces a section

$$
\widetilde{\mathcal{D}}_{\varrho} \in \Gamma\left(\widetilde{\mathfrak{M}}_{(\aleph, J)}^{\varrho} ; \mathbb{E}_{\varrho}^{*} \otimes \pi_{\varrho}^{*} \pi_{\mathbb{P} F_{(\aleph, J)}^{*}} \mathrm{ev}_{0}^{*} T \mathbb{P}^{n}\right)
$$

where $\pi_{\varrho}=\pi_{\varrho-1} \circ \tilde{\pi}_{\varrho}$.

The inductive assumptions (I1)-(I3), with $\varrho-1$ replaced by $\varrho$, are clearly satisfied, while (I5), (I8), and (I9) follow from (2) of Lemma 3.3 and Corollary 3.4. On the other hand, by (I6), the domain of the immersion $\iota_{\varrho-1, \varrho}$ is

$$
\widetilde{\mathcal{M}}_{0, \varrho P}^{\rho_{\varrho}(\varrho-1)} \times \overline{\mathfrak{M}}_{0,\left(\aleph_{B}(\varrho), J_{B}(\varrho)\right)}=\widetilde{\mathcal{M}}_{0, \varrho_{P}} \times \overline{\mathfrak{M}}_{0,\left(\aleph_{B}(\varrho), J_{B}(\varrho)\right)},
$$

where $\widetilde{\mathcal{M}}_{0, \varrho_{P}} \longrightarrow \widetilde{\mathcal{M}}_{0, \varrho_{P}}^{0}$ is the blowup constructed in Section 2.4. By (I11), the normal bundle for the immersion $\iota_{\varrho-1, \varrho}$ is given by

$$
\begin{aligned}
& \mathcal{N}_{l-1, \varrho}=\bigoplus_{l \in \boldsymbol{N}_{P}(\varrho)} \bigoplus_{i \in\left[m_{l}\right]} \pi_{P}^{*} L_{\rho_{\varrho}(\varrho-1),(l, i)} \otimes \pi_{B}^{*} \pi_{(l, i)}^{*} L_{0} \oplus \pi_{P}^{*} \mathbb{E}_{\rho_{\varrho}(\varrho-1)}^{*} \otimes \pi_{B}^{*} \bigoplus \pi_{l \in \boldsymbol{N}_{S}(\varrho)}^{*} L_{0} \\
& =\bigoplus_{l \in \aleph_{P}(\varrho)} \bigoplus_{i \in\left[m_{l}\right]} \pi_{P}^{*} \mathbb{L} \otimes \pi_{B}^{*} \pi_{(l, i)}^{*} L_{0} \oplus \pi_{P}^{*} \mathbb{L} \otimes \pi_{B}^{*} \bigoplus_{l \in \aleph_{S}(\varrho)} \pi_{l}^{*} L_{0} \\
& =\pi_{P}^{*} \mathbb{L} \otimes \pi_{B}^{*} F_{\left(\aleph_{B}(\varrho), J_{B}(\varrho)\right)},
\end{aligned}
$$

where $\mathbb{L} \longrightarrow \widetilde{\mathcal{M}}_{0, \varrho_{P}}$ is the universal tangent line bundle constructed in Section 2.4. We also note that by $(I 10)$,

$$
\iota_{\varrho-1, \varrho}^{*}\left(\mathbb{E}_{\varrho-1}^{*} \otimes \pi_{\mathbb{P} F_{(\aleph, J)}}^{*} \operatorname{ev}_{0}^{*} T \mathbb{P}^{n}\right)=\pi_{P}^{*} \mathbb{L} \otimes \pi_{B}^{*} \pi_{\mathbb{P} F_{\left(\aleph_{B}(\varrho), J_{B}(\varrho)\right)}^{*}} \mathrm{ev}_{0}^{*} T \mathbb{P}^{n} .
$$

By ( $I 12)$, the differential of $\widetilde{\mathcal{D}}_{\varrho-1}$ in the normal direction to the immersion $\iota_{\varrho-1, \varrho}$ is given by

$$
\nabla \widetilde{\mathcal{D}}_{\varrho-1}=\pi_{P}^{*} \operatorname{id} \otimes \pi_{B}^{*} \mathcal{D}_{\left(\aleph_{B}(\varrho), J_{B}(\varrho)\right)} .
$$

Thus, if

$$
\iota_{\varrho, \varrho}: \mathbb{P} \mathcal{N}_{\iota_{\varrho-1, \varrho}} \approx \widetilde{\mathcal{M}}_{0, \varrho_{P}} \times \widetilde{\mathfrak{M}}_{0,\left(\aleph_{P}(\varrho), J_{B}(\varrho)\right)}^{0} \longrightarrow \widetilde{\mathfrak{M}}_{0, \varrho}^{\varrho} \subset \widetilde{\mathfrak{M}}_{0,(\aleph, J)}^{\varrho}
$$

is the immersion induced by $\iota_{\varrho-1, \varrho}$, then

$$
\begin{aligned}
\iota_{\varrho, \varrho}^{*} \widetilde{\mathcal{D}}_{\varrho}=\pi_{B}^{*} \widetilde{\mathcal{D}}_{0} \in \Gamma\left(\widetilde{\mathcal{M}}_{0, \varrho_{P}} \times \widetilde{\mathfrak{M}}_{0,\left(\aleph_{P}(\varrho), J_{B}(\varrho)\right)}^{0} ; \iota_{\varrho, \varrho}^{*}\left(\mathbb{E}_{\varrho}^{*} \otimes \pi_{\varrho}^{*} \pi_{\mathbb{P} F_{(\aleph, J)}}^{*} e_{0}^{*} T \mathbb{P}^{n}\right)\right) \\
=\Gamma\left(\widetilde{\mathcal{M}}_{0, \varrho P} \times \widetilde{\mathfrak{M}}_{0,\left(\aleph_{B}(\varrho), J_{B}(\varrho)\right)}^{0} ;\right. \\
\left.\pi_{B}^{*}\left(\gamma_{\left(\aleph_{B}(\varrho), J_{B}(\varrho)\right)}^{*} \otimes \pi_{\mathbb{P} F_{\left(\aleph_{B}(\varrho), J_{B}(\varrho)\right)}^{*}}^{*} \operatorname{ev}_{0}^{*} T \mathbb{P}^{n}\right)\right) .
\end{aligned}
$$


Lemmas 3.10 and 3.11 thus imply that the restriction of the section $\widetilde{\mathcal{D}}_{\varrho}$ to the exceptional divisor $\widetilde{\mathfrak{M}}_{0, \varrho}^{\varrho}$ is transverse to the zero set away from the subvarieties $\widetilde{\mathfrak{M}}_{0, \varrho^{*}}^{\varrho}$ with $\varrho^{*}>\varrho$. Thus, by the inductive assumption (I4) as stated above, (I4) is satisfied with $\varrho-1$ replaced by $\varrho$.

We now verify that the remaining inductive assumptions are satisfied. If $\varrho<\varrho^{*}$, but $\varrho \nprec \varrho^{*}$,

$$
\rho_{\varrho^{*}}(\varrho)=\rho_{\varrho^{*}}(\varrho-1) \quad \text { and } \quad \overline{\mathfrak{M}}_{0, \varrho^{*}}^{\varrho-1} \cap \overline{\mathfrak{M}}_{0, \varrho}^{\varrho-1}=\varnothing,
$$

by definition and by (I5), respectively. It then follows that

$$
\begin{gathered}
\iota_{\varrho, \varrho^{*}}=\iota_{\varrho-1, \varrho^{*}, \quad} \widetilde{\mathfrak{M}}_{0, \varrho^{*}}^{\varrho} \cap \widetilde{\mathfrak{M}}_{0, \varrho^{\prime}}^{\varrho}=\widetilde{\mathfrak{M}}_{0, \varrho^{*}}^{\varrho-1} \cap \widetilde{\mathfrak{M}}_{0, \varrho^{\prime}}^{\varrho-1} \quad \forall \varrho^{\prime}>\varrho, \\
\iota_{\varrho, \varrho^{*}}^{*} \mathbb{E}_{\varrho}=\iota_{\varrho-1, \varrho^{*}}^{*} \mathbb{E}_{\varrho-1}, \quad \mathcal{N}_{\iota_{\varrho, \varrho^{*}}}=\mathcal{N}_{\iota_{\varrho-1, \varrho^{*}}}, \quad \text { and } \quad \nabla \widetilde{\mathcal{D}}_{\varrho}=\nabla \widetilde{\mathcal{D}}_{\varrho-1} .
\end{gathered}
$$

Thus, the inductive assumptions (I6), (I7), and (I10)-(I12), as stated above, imply the corresponding statements with $\varrho-1$ replaced by $\varrho$.

Suppose that $\varrho \prec \varrho^{*}$. By (I6) and (1) of Lemma 3.3, the domain of the immersion $\iota_{\varrho, \varrho^{*}}$ induced by the immersion $\iota_{\varrho-1, \varrho^{*}}$ is the blowup of

$$
\widetilde{\mathcal{M}}_{0, \varrho_{P}^{*}}^{\rho_{\varrho^{*}}(\varrho-1)} \times \overline{\mathfrak{M}}_{0,\left(\aleph_{B}\left(\varrho^{*}\right), J_{B}\left(\varrho^{*}\right)\right)}
$$

along the preimage of $\overline{\mathfrak{M}}_{0, \varrho}^{\varrho-1}$ under $\iota_{\varrho-1, \varrho^{*}}$ in

$$
\pi_{\rho_{\varrho^{*}(\varrho-1)} \times \mathrm{id}:} \widetilde{\mathcal{M}}_{0, \varrho_{P}^{*}}^{\rho_{Q^{*}}(\varrho-1)} \times \overline{\mathfrak{M}}_{0,\left(\aleph_{B}\left(\varrho^{*}\right), J_{B}\left(\varrho^{*}\right)\right)} \longrightarrow \widetilde{\mathcal{M}}_{0, \varrho_{P}^{*}}^{0} \times \overline{\mathfrak{M}}_{0,\left(\aleph_{B}\left(\varrho^{*}\right), J_{B}\left(\varrho^{*}\right)\right)}
$$

By (I7), this preimage is

$$
\left(\bigcup_{\rho \in \mathcal{A}_{0}\left(\varrho^{*} ; \varrho\right)} \widetilde{\mathcal{M}}_{0, \rho}^{\rho_{\varrho^{*}}(\varrho-1)}\right) \times \overline{\mathfrak{M}}_{0,\left(\aleph_{B}\left(\varrho^{*}\right), J_{B}\left(\varrho^{*}\right)\right)} .
$$

By the last paragraph of Section 2.4 and the second paragraph after Lemma 3.10,

$$
\widetilde{\mathcal{M}}_{0, \rho_{1}}^{\rho_{Q^{*}}(\varrho-1)} \cap \widetilde{\mathcal{M}}_{0, \rho_{2}}^{\rho_{Q^{*}}(\varrho-1)}=\varnothing \quad \forall \rho_{1}, \rho_{2} \in \mathcal{A}_{0}\left(\varrho^{*} ; \varrho\right), \rho_{1} \neq \rho_{2} .
$$

Thus, by the construction of Section 2.4, the blowup of $\widetilde{\mathcal{M}}_{0, \varrho_{P}^{*}}^{\rho_{\varrho^{*}}(\varrho-1)}$ along

$$
\bigcup_{\rho \in \mathcal{A}_{0}\left(\varrho^{*} ; \varrho\right)} \widetilde{\mathcal{M}}_{0, \rho}^{\rho_{Q^{*}}(\varrho-1)}
$$


is $\widetilde{\mathcal{M}}_{0, \varrho_{P}^{*}}^{\rho_{Q^{*}}(\varrho)}$, as needed for the inductive statement (I6), with $\varrho-1$ replaced by $\varrho$. For the same reasons, $(I 10),(2-13)$, and (3-7) imply that

$$
\begin{aligned}
\iota_{\varrho, \varrho^{*}}^{*} \mathbb{E}_{\varrho} & =\iota_{\varrho-1, \varrho^{*}}^{*} \mathbb{E}_{\varrho-1} \otimes \iota_{\varrho, \varrho^{*}}^{*} \mathcal{O}\left(\widetilde{\mathfrak{M}}_{0, \varrho}^{\varrho}\right) \\
& =\pi_{P}^{*} \mathbb{E}_{\rho_{\varrho^{*}}(\varrho-1)} \otimes \bigotimes_{\rho \in \mathcal{A}_{0}\left(\varrho^{*} ; \varrho\right)} \pi_{P}^{*} \mathcal{O}\left(\widetilde{\mathcal{M}}_{0, \rho}^{\rho}\right)=\pi_{P}^{*} \mathbb{E}_{\rho_{Q^{*}}(\varrho)} .
\end{aligned}
$$

Thus, the inductive statement $(I 10)$, with $\varrho-1$ replaced by $\varrho$, is satisfied. The assumption (I7) is checked similarly, using (3) of Lemma 3.3.

We next determine the normal bundle for the immersion $\iota_{\varrho, \varrho^{*}}$. By the construction of Section 2.4, the restrictions of the line bundles $L_{\rho_{Q^{*}}(\varrho-1),(l, i)}$ and $\mathbb{E}_{\rho_{Q^{*}(\varrho-1)}}$ to the complement of the exceptional divisors in $\widetilde{\mathcal{M}}_{0^{Q^{*}} \varrho_{P}^{*}\left(\varrho^{(-1)}\right.}$ are $\pi_{\rho_{Q^{*}}^{*}(\varrho-1)} L_{0,(l, i)}$ and $\pi_{\rho_{Q^{*}(\varrho-1)}}^{*} \mathbb{E}_{0}$. Thus, by the last statement of Lemma 3.10, (I11), and the inductive assumptions (I1) above and in Section 2.4,

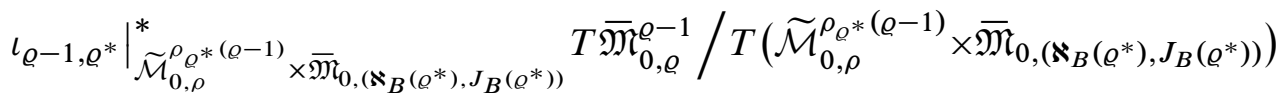

$$
\begin{aligned}
& =\bigoplus_{l \in \aleph_{P}\left(\varrho^{*}\right)-\aleph_{P}(\varrho)} \bigoplus_{i \in\left[m_{l}^{*}\right]} \pi_{P}^{*} L_{\rho_{Q^{*}}(\varrho-1),(l, i)} \otimes \pi_{B}^{*} \pi_{(l, i)}^{*} L_{0} \\
& \oplus \bigoplus_{l \in \aleph_{P}(\varrho)} \bigoplus_{i \in\left[m_{l}^{*}\right]-I_{l, P}^{*}} \pi_{P}^{*} L_{\rho^{*}}(\varrho-1),(l, i) \otimes \pi_{B}^{*} \pi_{(l, i)}^{*} L_{0}
\end{aligned}
$$

for all $\rho \in \mathcal{A}_{0}\left(\varrho^{*} ; \varrho\right)$ as in the statement of Lemma 3.10. Let

$$
I_{P}(\rho)=\left\{(l, i): l \in \aleph_{P}(\varrho), i \in I_{l, P}^{*}\right\} .
$$

From Lemma 3.5, we then obtain

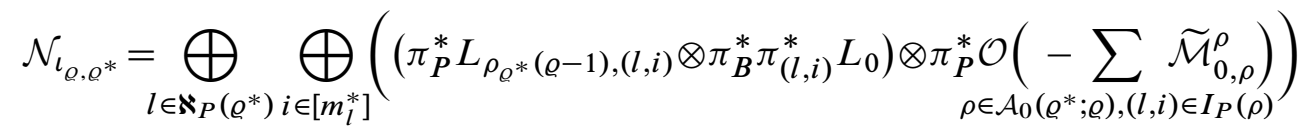

$$
\begin{aligned}
& \left.\oplus \pi_{P}^{*} \mathbb{E}_{\rho_{Q^{*}}(\varrho-1)}^{*} \otimes \pi_{B}^{*} \bigoplus_{l \in \mathbb{N}_{S}\left(\varrho^{*}\right)} \pi_{l}^{*} L_{0} \otimes \pi_{P}^{*} \mathcal{O}\left(-\sum_{\rho \in \mathcal{A}_{0}\left(\varrho^{*} ; \varrho\right)} \widetilde{\mathcal{M}}_{0, \rho}^{\rho}\right)\right) \\
& =\bigoplus_{l \in \boldsymbol{N}_{P}\left(\varrho^{*}\right)} \bigoplus_{i \in\left[m_{l}^{*}\right]}\left(\pi_{P}^{*} L_{\rho_{Q^{*}}(\varrho),(l, i)} \otimes \pi_{B}^{*} \pi_{(l, i)}^{*} L_{0}\right) \oplus \pi_{P}^{*} \mathbb{E}_{\rho_{\varrho^{*}}(\varrho)}^{*} \otimes \pi_{B}^{*} \bigoplus_{l \in \boldsymbol{\aleph}_{S}\left(\varrho^{*}\right)} \pi_{l}^{*} L_{0} .
\end{aligned}
$$

The last equality above follows from (2-12) by the same argument as in the previous paragraph. We have thus verified that the inductive assumption (I11), with $\varrho-1$ replaced by $\varrho$, is satisfied. Finally, the inductive assumption (I12) and the continuity of the two bundle sections involved in the identity in $(I 12)$, with $\varrho-1$ replaced by $\varrho$, imply ( $I 12)$ with $\varrho-1$ replaced by $\varrho$. 
We conclude this construction after the blowup at the $\varrho_{\max }$ step. Let

$$
\widetilde{\mathfrak{M}}_{0,(\aleph, J)}\left(\mathbb{P}^{n}, d\right)=\widetilde{\mathfrak{M}}_{0,(\aleph, J)}^{\varrho_{\max }}, \quad \widetilde{\mathbb{E}}=\mathbb{E}_{\varrho_{\max }}, \quad \widetilde{\mathcal{D}}_{(\aleph, J)}=\widetilde{\mathcal{D}}_{\varrho_{\max }} .
$$

By the inductive assumption (I4), applied with $\varrho-1$ replaced by $\varrho_{\max }$, the section $\widetilde{\mathcal{D}}_{(\aleph, J)}$ is transverse to the zero set. As in the previous two subsections, the final result of this blowup construction is independent of the order $<$ chosen to extend the partial ordering $\prec$ on $\mathcal{A}_{0}(\boldsymbol{\aleph} ; d, J)$, as can be seen from (I5).

\section{A blowup of a moduli space of genus-one maps}

\subsection{Idealized blowups and immersions}

In this section we describe the main blowup construction of this paper. This is the sequential idealized blowup construction for $\overline{\mathfrak{M}}_{1, k}\left(\mathbb{P}^{n}, d\right)$ with the initial data and the inductive step specified in Sections 4.2 and 4.3, respectively. This construction is outlined in Sections 1.2 and 1.3.

In contrast to the situations in Sections 2 and 3, the variety $\overline{\mathfrak{M}}_{1, k}\left(\mathbb{P}^{n}, d\right)$ is singular. In order to describe the structure of $\overline{\mathfrak{M}}_{1, k}\left(\mathbb{P}^{n}, d\right)$, we introduce the notion of idealized normal bundle for an immersion. Recall that the domain of an immersion is assumed to be a smooth variety.

Definition 4.1 Suppose $\overline{\mathfrak{M}}$ is a variety and $\iota_{X}: X \longrightarrow \overline{\mathfrak{M}}$ is an immersion. An idealized normal bundle for the immersion $\iota_{X}$ is a vector bundle $\mathcal{N}_{\iota_{X}}^{\text {ide }}$ over $X$ such that $\mathcal{N}_{\iota_{X}} \subset \mathcal{N}_{\iota_{X}}^{\text {ide }}$.

Remark An idealized normal bundle is of course not unique; an idealized normal bundle plus any other vector bundle is still an idealized normal bundle. If the image of $\iota_{X}$ is an irreducible component of $\overline{\mathfrak{M}}$, an idealized normal bundle of the smallest possible rank still need not be unique; it can be twisted by any divisor in $X$ disjoint from the preimage under $\iota_{X}$ of the other components of $\overline{\mathfrak{M}}$. For each of the immersions we encounter in the next subsection, there is a natural choice for $\mathcal{N}_{l_{X}}^{\text {ide }}$. These idealized normal bundles also transform in a natural way under blowups and proper immersions, as described in Lemma 4.3 below.

Suppose $\overline{\mathfrak{M}}$ is a variety, $Z$ is a smooth subvariety of $\overline{\mathfrak{M}}$, and $\mathcal{N}_{\iota Z}^{\text {ide }}$ is an idealized normal bundle for the embedding $\iota_{Z}$ of $Z$ into $\overline{\mathfrak{M}}$. Let

$$
\mathcal{E}_{Z} \equiv \mathbb{P} \mathcal{N}_{\iota_{Z}} \subset \mathrm{Bl}_{Z} \overline{\mathfrak{M}}
$$


be the exceptional divisor for the blowup of $\overline{\mathfrak{M}}$ along $Z$. We denote by $\mathrm{Bl}_{Z}^{\text {ide }} \overline{\mathfrak{M}}$ the variety obtained by identifying $\mathrm{Bl}_{Z} \overline{\mathfrak{M}}$ with

$$
\mathcal{E}_{Z}^{\text {ide }} \equiv \mathbb{P} \mathcal{N}_{l Z}^{\text {ide }}
$$

along $\mathcal{E}_{Z}$. We will call

$$
\pi^{\text {ide }}: \mathrm{Bl}_{Z}^{\text {ide }} \overline{\mathfrak{M}} \longrightarrow \overline{\mathfrak{M}}
$$

the idealized blowup of $\overline{\mathfrak{M}}$ along $Z$ and

$$
\mathcal{E}_{Z}^{\text {ide }} \subset \mathrm{Bl}_{Z}^{\text {ide }} \overline{\mathfrak{M}}
$$

the idealized exceptional divisor for $\pi^{\text {ide }}$. (Caution: the idealized exceptional divisor is not necessarily a divisor!) More generally, we will call

$$
\pi: \widetilde{\mathfrak{M}} \longrightarrow \overline{\mathfrak{M}}
$$

an idealized blowup of $\overline{\mathfrak{M}}$ if $\pi$ is a composition of idealized blowups along smooth subvarieties. In practice, idealized blowup is simply a convenient term. In the inductive assumption (I1) in Section 4.3 below, it can be replaced by morphism of varieties, as the remaining inductive assumptions describe all the relevant properties of this morphism. Let

$$
\gamma_{Z} \longrightarrow \mathcal{E}_{Z}^{\text {ide }}
$$

be the tautological line bundle. Note that the normal bundle of $\mathcal{E}_{Z} \subset \mathcal{E}_{Z}^{\text {ide }}$ in

$$
\operatorname{Pr}_{Z} \overline{\mathfrak{M}}=\mathrm{Bl}_{Z} \overline{\mathfrak{M}}
$$

is $\left.\gamma_{Z}\right|_{\mathcal{E}_{Z}}$. This observation implies the first statement of Lemma 4.3 below.

Our strategy is as follows. We begin with a space with a properly self-intersecting collection of immersions, each with an idealized normal bundle. These are the immersions $\iota_{\sigma}$ with $\sigma \in \mathcal{A}_{1}(d, k)$ defined in Section 1.2; their images are the subvarieties $\overline{\mathfrak{M}}_{1, \sigma}\left(\mathbb{P}^{n}, d\right)$ of $\overline{\mathfrak{M}}_{1, k}\left(\mathbb{P}^{n}, d\right)$. The idealized normal bundle for the immersion $\iota_{\sigma}$ is the direct sum of the deformation spaces of the nodes between the contracted genus-one curve and the noncontracted genus-zero curves that are identified by $\iota_{\sigma}$. At each stage, one of our immersions is an embedding, and we blow it up, replacing it with its idealized exceptional divisor. The exceptional divisor of the blowup of the main component is the intersection of the new main component with the idealized exceptional divisor. Then after each step, we have a new properly self-intersecting collection of immersions. Moreover, there is a natural idealized normal bundle for each of the proper transforms of the immersions we have "yet to blow up".

We now say this more explicitly. Lemmas 4.2 and 4.3 below are direct extensions of Corollary 3.4 and Lemma 3.5. The first lemma states that if we have a properly 
self-intersecting collection of immersions, one of which is an embedding, then upon blowing up the embedding, we still have a properly self-intersecting collection of immersions. It is immediate from the definition of "properly self-intersecting", by checking in local coordinates.

The second part of the second lemma follows from Lemma 3.5 with only one change. Instead of writing

$$
\mathcal{N}_{\iota_{X}}=\bigoplus_{i \in I} L_{i} \quad \text { and } \quad \mathcal{N}_{\operatorname{Pr}_{Z} \iota_{X}}=\bigoplus_{i \in I}\left(\pi^{*} L_{i} \otimes \bigotimes_{i \in I_{\varrho}} \mathcal{O}\left(-E_{\varrho}\right)\right)
$$

as in the statement of Lemma 3.5, we are saying that if there is a natural inclusion $\mathcal{N}_{l_{X}} \subset \bigoplus_{i \in I} L_{i}$, then we get a natural inclusion

$$
\mathcal{N}_{\operatorname{Pr}_{Z l_{X}}} \subset \bigoplus_{i \in I}\left(\pi^{*} L_{i} \otimes \bigotimes_{i \in I_{\varrho}} \mathcal{O}\left(-E_{\varrho}\right)\right)
$$

The vector bundles on the right sides of the two expressions in (4-1) are the original idealized normal bundle and the new idealized normal bundle.

Lemma 4.2 Suppose $\overline{\mathfrak{M}}$ is a variety, $\left\{\iota_{\sigma}: X_{\sigma} \longrightarrow \overline{\mathfrak{M}}\right\}_{\sigma \in \mathcal{A}}$ is a properly self-intersecting collection of immersions, and $\sigma \in \mathcal{A}$ is such that $\iota_{\sigma}$ is an embedding. If $\mathcal{N}_{\iota_{\sigma}}^{\text {ide }}$ is an idealized normal bundle for $\iota_{\sigma}$, then

$$
\left\{\operatorname{Pr}_{\operatorname{Im} \iota_{\sigma}} \iota_{\sigma^{\prime}}\right\}_{\sigma^{\prime} \in \mathcal{A}-\{\sigma\}} \cup\left\{\iota_{\mathcal{E}_{\operatorname{Im} \iota \sigma}^{\text {ide }}}\right\}
$$

is a properly self-intersecting collection of immersions into $\mathrm{B}_{\operatorname{Im} \iota_{\sigma}}^{\text {ide }} \overline{\mathfrak{M}}$.

Lemma 4.3 If $\overline{\mathfrak{M}}$ is a variety, $Z$ is a smooth subvariety of $\overline{\mathfrak{M}}$, and $\mathcal{N}_{\iota_{Z}}^{\text {ide }}$ is an idealized normal bundle for ${ } Z$, then

$$
\mathcal{N}_{\iota_{\mathcal{E}_{Z}^{\text {ide }}}^{\text {ide }}}^{\text {ing }}=\gamma_{Z}
$$

is an idealized normal bundle for the immersion $\iota_{\mathcal{Z}}^{\text {ide }}$. Suppose in addition that $\iota_{X}, \mathcal{A}$, $Z_{\varrho}$, and $E_{\varrho}$ are as in Lemma 3.5 and $\mathcal{N}_{\iota_{X}}^{\text {ide }}$ is an idealized normal bundle for $\iota_{X}$. If there exist a splitting

$$
\mathcal{N}_{l_{X}}^{\text {ide }}=\bigoplus_{i \in I} L_{i} \longrightarrow X
$$

and a subset $I_{\varrho}$ of $I$ for each $\varrho \in \mathcal{A}$ such that (3-1) holds, then

$$
\mathcal{N}_{\operatorname{Pr}_{Z} \iota_{X}}^{\text {ide }}=\bigoplus_{i \in I}\left(\pi^{*} L_{i} \otimes \bigotimes_{i \in I_{\varrho}} \mathcal{O}\left(-E_{\varrho}\right)\right)
$$

is an idealized normal bundle for the immersion $\operatorname{Pr}_{Z} \iota_{X}$. 
Definition 4.4 Suppose $\overline{\mathfrak{M}}$ is a variety, $\iota_{X}: X \longrightarrow \overline{\mathfrak{M}}$ is an immersion, $\overline{\mathfrak{M}}^{0}$ is a subvariety in $\overline{\mathfrak{M}}$, and $T C \overline{\mathfrak{M}}^{0} \subset T \overline{\mathfrak{M}}$ is the tangent cone of $\overline{\mathfrak{M}}^{0}$ in $\overline{\mathfrak{M}}\left(T C \overline{\mathfrak{M}}^{0}\right.$ not necessarily reduced). The subvariety $\overline{\mathfrak{M}}^{0}$ is proper relative to $\iota_{X}$ if

$$
d \iota_{X} T C \iota_{X}^{-1}\left(\overline{\mathfrak{M}}^{0}\right)=\iota_{X}^{*} T C \overline{\mathfrak{M}}^{0} \cap \operatorname{Im} d \iota_{X} \subset \iota_{X}^{*} T \overline{\mathfrak{M}}
$$

and the map

$$
\left.\iota_{X}^{*} T C \overline{\mathfrak{M}}^{0}\right|_{\iota_{X}^{-1}\left(\overline{\mathfrak{M}}^{0}\right)} /\left.\operatorname{Im} d \iota_{X}\right|_{T C_{X} \iota^{-1}\left(\overline{\mathfrak{M}}^{0}\right)} \longrightarrow \iota_{X}^{*} T \overline{\mathfrak{M}} / \operatorname{Im} d \iota_{X} \subset \mathcal{N}_{\iota_{X}}^{\text {ide }}
$$

induced by inclusions is injective, with its image being reduced.

The left-hand side of (4-2) denotes the family of cones over $\iota_{X}^{-1}\left(\overline{\mathfrak{M}}^{0}\right)$ such that for each $x \in \iota_{X}^{-1}\left(\overline{\mathfrak{M}}^{0}\right)$

$$
\left.\iota_{X}^{*} T C \overline{\mathfrak{M}}^{0}\right|_{\iota_{X}^{-1}\left(\overline{\mathfrak{M}}^{0}\right)} /\left.\operatorname{Im} d \iota_{X}\right|_{\left.T C \iota_{X}^{-1}\left(\overline{\mathfrak{M}}^{0}\right)\right|_{X}}
$$

is the quotient by the minimal vector subspace of $\left.\operatorname{Im} d \iota_{X}\right|_{x}=d \iota_{X}\left(T_{X} X\right)$ containing the cone $\left.\operatorname{Im} d \iota_{X}\right|_{T_{X} C \iota_{X}^{-1}\left(\overline{\mathfrak{M}}^{0}\right)}$. If $T C \iota_{X}^{-1}\left(\overline{\mathfrak{M}}^{0}\right)$ is a vector bundle, the two conditions in Definition 4.4 are equivalent.

If $\overline{\mathfrak{M}}^{0}$ is a subvariety of $\overline{\mathfrak{M}}$ which is proper relative to an immersion $\iota_{X}: X \longrightarrow \overline{\mathfrak{M}}$, we denote by

$$
\mathcal{N}_{\iota_{X} \mid \overline{\mathfrak{M}}^{0}} \subset \iota_{X}^{*} T \overline{\mathfrak{M}} / \operatorname{Im} d \iota_{X} \subset \mathcal{N}_{\iota_{X}}^{\text {ide }}
$$

the image of the homomorphism (4-2). We will call $\mathcal{N}_{\iota_{X} \mid \overline{\mathfrak{M}}^{0}}$ the normal cone of $\left.{ }^{\iota}\right|_{\iota_{X}^{-1}\left(\overline{\mathfrak{M}}^{0}\right)}$ in $\overline{\mathfrak{M}}^{0}$.

Lemma 4.5 Suppose $\overline{\mathfrak{M}}$ is a variety, $\iota_{X}: X \longrightarrow \overline{\mathfrak{M}}$ is an immersion with an idealized normal bundle $\mathcal{N}_{\iota_{X}}^{\text {ide }}, \overline{\mathfrak{M}}^{0}$ is a subvariety of $\overline{\mathfrak{M}}$ which is proper relative to $\iota_{X}$, and

$$
\mathcal{Z} \subset \overline{\mathcal{Z}} \equiv \iota_{X}^{-1}\left(\overline{\mathfrak{M}}^{0}\right)
$$

is such that $\mathcal{N}_{l_{X} \mid \overline{\mathfrak{M}}^{0}}$ is the closure of $\left.\mathcal{N}_{l_{X} \mid \overline{\mathfrak{M}}^{0}}\right|_{\mathcal{Z}}$ in $\mathcal{N}_{l_{X}}^{\text {ide }}$.

(1) If $X$ is a smooth subvariety of $\overline{\mathfrak{M}}$, then $\operatorname{Pr}_{X} \overline{\mathfrak{M}}^{0}$ is proper relative to the immer$\operatorname{sion} \iota^{\mathcal{E}_{X}^{\text {ide }}}$,

$$
\mathcal{E}_{X}^{\text {ide }} \cap \operatorname{Pr}_{X} \overline{\mathfrak{M}}^{0} \subset \mathcal{E}_{X}
$$

is the closure of $\mathbb{P} \mathcal{N}_{\iota_{X} \mid \overline{\mathfrak{M}}^{0}} \mid \mathcal{Z}$ in $\mathcal{E}_{X}^{\text {ide }}$, and

$$
\mathcal{N}_{l_{\mathcal{E}_{X}^{\text {ide }}} \mid \operatorname{Pr}_{X} \overline{\mathfrak{M}}^{0}}=\left.\gamma_{X}\right|_{\mathcal{E}_{X}^{\text {ide }} \cap \operatorname{Pr}_{X} \overline{\mathfrak{M}}^{0}}
$$

(2) If $Z$ is a smooth subvariety of $\overline{\mathfrak{M}}$ disjoint from $\iota_{X}(\mathcal{Z})$ and $\mathcal{N}_{\iota_{Z}}^{\text {ide }}$ is an idealized normal bundle for $\iota_{Z}$, then $\operatorname{Pr}_{Z} \overline{\mathfrak{M}}^{0}$ is a proper subvariety of the blowup $\mathrm{Bl}_{Z}^{\text {ide }} \overline{\mathfrak{M}}$ 
relative to the immersion $\operatorname{Pr}_{Z} \iota_{X}$ and $\mathcal{N}_{\operatorname{Pr}_{Z} \iota_{X} \mid \operatorname{Pr}} \overline{\mathfrak{M}}^{0}$ is the closure of $\left.\mathcal{N}_{\iota_{X} \mid \overline{\mathfrak{M}}^{0}}\right|_{\mathcal{Z}}$ in $\mathcal{N}_{\operatorname{Pr}_{Z} \iota_{X}}^{\text {ide }}$.

The first part of (1) essentially follows from the universal property of blowing up: if $\overline{\mathfrak{M}}$ is blown up along $Z$, then the proper transform of $\overline{\mathfrak{M}}^{0}$ in $\overline{\mathfrak{M}}$ (the scheme-theoretic closure of $\overline{\mathfrak{M}}^{0}-Z$ in the blowup) is the blowup of $\overline{\mathfrak{M}}^{0}$ along $\overline{\mathfrak{M}}^{0} \cap Z$, and the normal bundle to the exceptional divisor in $\mathrm{Bl}_{\overline{\mathfrak{M}}^{0} \cap Z^{\mathfrak{M}^{0}}}$ is the restriction of the normal bundle of the exceptional divisor in $\mathrm{Bl}_{Z} \overline{\mathfrak{M}}$. The statement (1) is the etale-local version of this. Part (2) is clear by working in local coordinates.

\subsection{Preliminaries}

In this subsection, we state a number of known facts concerning the moduli space $\overline{\mathfrak{M}}_{1, k}\left(\mathbb{P}^{n}, d\right)$ that insure that the inductive requirements of the next subsection are satisfied at the initial stage of the inductive construction. Lemmas 4.6-4.9, with the exception of one statement, are well-known in Gromov-Witten theory and are obtained similarly to Lemmas 3.6 and 3.7. We show that the last statement of Lemma 4.7 is simply a reinterpretation of a standard fact concerning moduli spaces of stable maps.

Let $\left(\mathcal{A}_{1}(d, k), \prec\right)$ be the partially ordered set of triples described in Section 1.2. It has a unique minimal element and a unique maximal element:

$$
\sigma_{\min }=(1 ; \varnothing,[k]) \quad \text { and } \quad \sigma_{\max }=(d ;[k], \varnothing) .
$$

Let $<$ be an order on $\mathcal{A}_{1}(d, k)$ extending the partial ordering $\prec$. For every $\sigma \in A_{1}(d, k)$, we define

$$
\sigma-1 \in\{0\} \sqcup \mathcal{A}_{1}(d, k)
$$

as in (2-7). For each element $\sigma=\left(m ; J_{P}, J_{B}\right)$ of $\mathcal{A}_{1}(d, k)$, let

$$
\overline{\mathfrak{M}}_{1, \sigma}^{0} \equiv \overline{\mathfrak{M}}_{1, \sigma}\left(\mathbb{P}^{n}, d\right) \subset \overline{\mathfrak{M}}_{1, k}^{0} \equiv \overline{\mathfrak{M}}_{1, k}\left(\mathbb{P}^{n}, d\right)
$$

be the subvarieties defined in Section 1.2.

Warning Note that $\overline{\mathfrak{M}}_{1, k}^{0}$ denotes the entire moduli space $\overline{\mathfrak{M}}_{1, k}\left(\mathbb{P}^{n}, d\right)$ and not the main component $\overline{\mathfrak{M}}_{1, k}^{0}\left(\mathbb{P}^{n}, d\right)$. Similarly to Sections 2 and 3, the superscript 0 indicates the $0-$ th stage in the blowup process.

Lemma 4.6 If $\sigma_{1}=\left(m_{1} ; J_{1 ; P}, J_{1 ; B}\right)$ and $\sigma_{2}=\left(m_{2} ; J_{2 ; P}, J_{2 ; B}\right)$ are elements of $\mathcal{A}_{1}(d, k), \sigma_{1} \neq \sigma_{2}, \sigma_{1} \nprec \sigma_{2}$, and $\sigma_{2} \nprec \sigma_{1}$, then

$$
\overline{\mathfrak{M}}_{1, \sigma_{1}}^{0} \cap \overline{\mathfrak{M}}_{1, \sigma_{2}}^{0} \subset \overline{\mathfrak{M}}_{1, \tilde{\sigma}\left(\sigma_{1}, \sigma_{2}\right)}^{0},
$$

where

$$
\tilde{\sigma}\left(\sigma_{1}, \sigma_{2}\right)=\left(\min \left(m_{1}, m_{2}\right) ; J_{1 ; P} \cap J_{2 ; P}, J_{1 ; B} \cup J_{2 ; B}\right) \text {. }
$$


With $\sigma$ as above, we define

$$
I_{P}(\sigma)=\aleph_{B}(\sigma)=[m], \quad J_{P}(\sigma)=J_{P}, \quad J_{B}(\sigma)=J_{B}, \quad G_{\sigma}=S_{m} .
$$

As in Section 1.2, we denote by

$$
\iota_{0, \sigma}: \overline{\mathcal{M}}_{1,\left(I_{P}(\sigma), J_{P}(\sigma)\right)}^{0} \times \overline{\mathfrak{M}}_{0,\left(\aleph_{B}(\sigma), J_{B}(\sigma)\right)} \longrightarrow \overline{\mathfrak{M}}_{1, \sigma}^{0} \subset \overline{\mathfrak{M}}_{1, k}^{0},
$$

where

$$
\overline{\mathfrak{M}}_{0,\left(\aleph_{B}(\sigma), J_{B}(\sigma)\right)}=\overline{\mathfrak{M}}_{0,\left(\aleph_{B}(\sigma), J_{B}(\sigma)\right)}\left(\mathbb{P}^{n}, d\right),
$$

the natural node-identifying map and by

$$
\bar{\iota}_{0, \sigma}:\left(\overline{\mathcal{M}}_{1,\left(I_{P}(\sigma), J_{P}(\sigma)\right)}^{0} \times \overline{\mathfrak{M}}_{0,\left(\aleph_{B}(\sigma), J_{B}(\sigma)\right)}\right) / G_{\sigma} \longrightarrow \overline{\mathfrak{M}}_{1, k}^{0}
$$

the induced immersion. Let

$\pi_{P}, \pi_{B}: \overline{\mathcal{M}}_{1,\left(I_{P}(\sigma), J_{P}(\sigma)\right)}^{0} \times \overline{\mathfrak{M}}_{0,\left(\aleph_{B}(\sigma), J_{B}(\sigma)\right)}^{\longrightarrow} \overline{\mathcal{M}}_{1,\left(I_{P}(\sigma), J_{P}(\sigma)\right)}^{0}, \overline{\mathfrak{M}}_{0,\left(\aleph_{P}(\sigma), J_{B}(\sigma)\right)}$

be the two projection maps.

Lemma 4.7 If $d, n \in \mathbb{Z}^{+}$and $k \in \overline{\mathbb{Z}}^{+},\left\{\iota_{0, \sigma}\right\}_{\sigma \in \mathcal{A}_{1}(d, k)}$ and $\left\{\bar{\iota}_{0, \sigma}\right\}_{\sigma \in \mathcal{A}_{1}(d, k)}$ are properly self-intersecting collections of immersions. If $\sigma^{*}=\left(m^{*} ; J_{P}^{*}, J_{B}^{*}\right) \in \mathcal{A}_{1}(d, k)$,

$$
\operatorname{Im}^{s} \bar{\iota}_{0, \sigma^{*}} \subset \bigcup_{\sigma^{\prime}<\sigma^{*}} \overline{\mathfrak{M}}_{1, \sigma^{\prime}} \quad \text { and } \quad \mathcal{N}_{\iota_{0, \sigma^{*}}}^{\mathrm{ide}}=\bigoplus_{i \in\left[m^{*}\right]} \pi_{P}^{*} L_{i} \otimes \pi_{B}^{*} \pi_{i}^{*} L_{0}
$$

is an idealized normal bundle for $\iota_{0, \sigma^{*}}$.

We deduce the last claim of this lemma from the deformation-obstruction exact sequence (24.2) in [10] as follows. Suppose

$$
[\Sigma, u]=\iota_{0, \sigma^{*}}\left(\left[\Sigma_{P}\right] \times\left[\Sigma_{B}, u_{B}\right]\right) \in \overline{\mathfrak{M}}_{1, \sigma^{*}}^{0},
$$

where

$$
\left[\Sigma_{B}, u_{B}\right]=\left(\left[\Sigma_{i}, u_{i}\right]\right)_{i \in\left[m^{*}\right]} \in \overline{\mathfrak{M}}_{0,\left(\aleph_{B}\left(\sigma^{*}\right), J_{B}\left(\sigma^{*}\right)\right)} .
$$

By [10, (24.2)], there exists a natural homomorphism

$$
j_{\Sigma, u}:\left.T \overline{\mathfrak{M}}_{1, k}\left(\mathbb{P}^{n}, d\right)\right|_{[\Sigma, u]}=\operatorname{Def}(\Sigma, u) \longrightarrow \operatorname{Def}(\Sigma),
$$

where $\operatorname{Def}(\Sigma, u)$ and $\operatorname{Def}(\Sigma)$ denote the deformations of the stable-map pair $(\Sigma, u)$ and the deformations of the curve $\Sigma$ (with its marked points), respectively. As $[\Sigma, u]$ is considered as the image of $\left[\Sigma_{P}\right] \times\left[\Sigma_{B}, u_{B}\right]$ under $\iota_{0, \sigma^{*}}$, there are $m^{*}$ distinguished nodes of $\Sigma$. These are the nodes of $\Sigma$ that do not correspond to either the nodes of $\Sigma_{\boldsymbol{P}}$ or the nodes of any of the curves $\Sigma_{i}$ with $i \in\left[m^{*}\right]$; see Figure 13 . Let

$$
\operatorname{Def}\left(\Sigma_{P}, \Sigma_{B}\right) \subset \operatorname{Def}(\Sigma)
$$


be the deformations of $\Sigma$ that do not smooth out the distinguished nodes of $\Sigma$. Since the smoothing of a given node of $\Sigma$ is parametrized by the tensor product of the tangent lines to the two branches of $\Sigma$ at the node, we have an exact sequence

$$
\left.0 \longrightarrow \operatorname{Def}\left(\Sigma_{P}, \Sigma_{B}\right) \longrightarrow \operatorname{Def}(\Sigma) \stackrel{j_{\Sigma}}{\longrightarrow} \mathcal{N}_{\iota_{0, \sigma^{*}}}^{\text {ide }}\right|_{[\Sigma, u]} \longrightarrow 0 .
$$

We denote by

$$
\left.\operatorname{Def}\left(\Sigma_{P},\left(\Sigma_{B}, u_{B}\right)\right) \subset T \overline{\mathfrak{M}}_{1, k}\left(\mathbb{P}^{n}, d\right)\right|_{[\Sigma, u]}=\operatorname{Def}(\Sigma, u)
$$

the kernel of the map

$$
j_{\Sigma} \circ j_{\Sigma, u}:\left.\operatorname{Def}(\Sigma, u) \longrightarrow \mathcal{N}_{\iota_{0, \sigma^{*}}}^{\text {ide }}\right|_{\left[\Sigma_{P}\right] \times\left[\Sigma_{B}, u_{B}\right]} .
$$

The space $\operatorname{Def}\left(\Sigma_{P},\left(\Sigma_{B}, u_{B}\right)\right)$ consists of deformations of $(\Sigma, u)$ that do not smooth out the $m^{*}$ distinguished nodes of $\Sigma$. Thus,

$$
\begin{aligned}
\operatorname{Def}\left(\Sigma_{P},\left(\Sigma_{B}, u_{B}\right)\right) & \approx \operatorname{Def}\left(\Sigma_{P}\right) \oplus \operatorname{Def}\left(\Sigma_{B}, u_{B}\right) \\
& =\left.\left.T \overline{\mathcal{M}}_{1,\left(I_{P}\left(\sigma^{*}\right), J_{P}\left(\sigma^{*}\right)\right)}^{0}\right|_{\left[\Sigma_{P}\right]} \oplus T \overline{\mathfrak{M}}_{0,\left(\aleph_{B}\left(\sigma^{*}\right), J_{B}\left(\sigma^{*}\right)\right)}\right|_{\left[\Sigma_{B}, u_{B}\right]} .
\end{aligned}
$$

The isomorphism from the right-hand side to the left-hand side is given by $d \iota_{0, \sigma^{*}}$. Thus, the homomorphism $j_{\Sigma} \circ j_{\Sigma, u}$ induces an injection

$$
\left.\left.\mathcal{N}_{\iota_{0, \sigma^{*}}}\right|_{[\Sigma, u]} \equiv T C \overline{\mathfrak{M}}_{1, k}\left(\mathbb{P}^{n}, d\right)\right|_{[\Sigma, u]} /\left.\operatorname{Im} d \iota_{0, \sigma^{*}} \longrightarrow \mathcal{N}_{\iota_{0, \sigma^{*}}}^{\text {ide }}\right|_{\left[\Sigma_{P}\right] \times\left[\Sigma_{B}, u_{B}\right]},
$$

as needed.
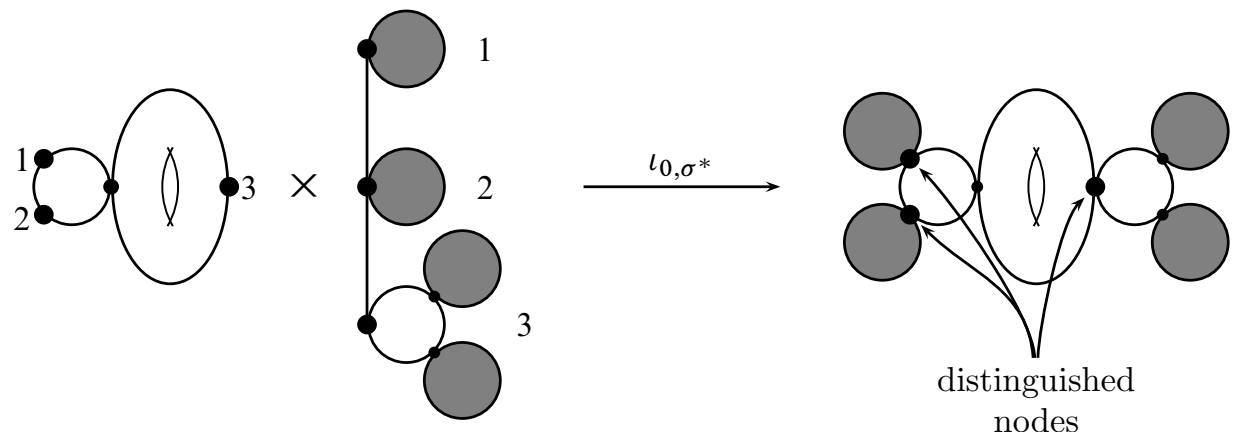

Figure 13: A point in the domain of $\iota_{0, \sigma^{*}}$ and its image in $\overline{\mathfrak{M}}_{1, k}\left(\mathbb{P}^{n}, d\right)$ 
Lemma 4.8 If $d, n, k$, and $\sigma^{*}$ are as in Lemma 4.7, $\sigma \in \mathcal{A}_{1}(d, k)$ is as above, and $\sigma \prec \sigma^{*}$, then

$$
\iota_{0, \sigma^{*}}^{-1}\left(\overline{\mathfrak{M}}_{1, \sigma}^{0}\right)=\left(\underset{\rho \in \mathcal{A}_{P}\left(\sigma^{*} ; \sigma\right)}{\bigcup} \overline{\mathcal{M}}_{1, \rho}^{0}\right) \times \overline{\mathfrak{M}}_{0,\left(\aleph_{B}\left(\sigma^{*}\right), J_{B}\left(\sigma^{*}\right)\right)},
$$

where $\quad \mathcal{A}_{P}\left(\sigma^{*} ; \sigma\right)=\left\{\rho=\left(I_{P} \sqcup J_{P},\left\{I_{k} \sqcup J_{k}: k \in K\right\}\right) \in \mathcal{A}_{1}\left(I_{P}\left(\sigma^{*}\right), J_{P}\left(\sigma^{*}\right)\right)\right.$ :

$$
\left.|K|+\left|I_{P}\right|=m\right\}
$$

and $\mathcal{A}_{1}\left(I_{P}\left(\sigma^{*}\right), J_{P}\left(\sigma^{*}\right)\right)$ and $\overline{\mathcal{M}}_{1, \rho}^{0} \equiv \overline{\mathcal{M}}_{1, \rho}$ are as in Section 2.2. Furthermore, if $\rho \in \mathcal{A}_{P}\left(\sigma^{*} ; \sigma\right)$ is as above,

$$
\begin{aligned}
\left.\iota_{0, \sigma^{*}}\right|_{\overline{\mathcal{M}}_{1, \rho}^{0} \times \overline{\mathfrak{M}}_{0,\left(\aleph_{B}\left(\sigma^{*}\right), J_{B}\left(\sigma^{*}\right)\right)} T \overline{\mathfrak{M}}_{1, \sigma}^{0} / T\left(\overline{\mathcal{M}}_{1, \rho}^{0} \times \overline{\mathfrak{M}}_{0,\left(\aleph_{B}\left(\sigma^{*}\right), J_{B}\left(\sigma^{*}\right)\right)}\right)} \\
=\bigoplus_{i \in I_{P}\left(\sigma^{*}\right)-I_{P}} \pi_{P}^{*} L_{i} \otimes \pi_{B}^{*} \pi_{i}^{*} L_{0} .
\end{aligned}
$$

Lemma 4.9 If $d, n, k, \sigma$, and $\sigma^{*}$ are as above, then

$$
\iota_{0, \sigma}^{-1}\left(\overline{\mathfrak{M}}_{1, \sigma^{*}}^{0}\right)=\overline{\mathcal{M}}_{1,\left(I_{P}(\sigma), J_{P}(\sigma)\right)}^{0} \times\left(\underset{\varrho \in \mathcal{A}_{B}\left(\sigma ; \sigma^{*}\right)}{\bigcup} \overline{\mathfrak{M}}_{0, \varrho}\right),
$$

where $\mathcal{A}_{B}\left(\sigma ; \sigma^{*}\right)=\left\{\varrho=\left(\left(\sigma_{l}\right)_{l \in \aleph_{B}(\sigma)}, J_{B}\right) \in \mathcal{A}_{0}\left(\aleph_{B}(\sigma) ; d, J_{B}(\sigma)\right):\left|\aleph_{B}(\varrho)\right|=m^{*}\right\}$,

and $\mathcal{A}_{0}\left(\aleph_{B}(\sigma) ; d, J_{B}(\sigma)\right), \aleph_{B}(\varrho)$, and $\overline{\mathfrak{M}}_{0, \varrho} \equiv \overline{\mathfrak{M}}_{0, \varrho}\left(\mathbb{P}^{n}, d\right)$ are as in Section 3.3. Furthermore, if $\varrho \in \mathcal{A}_{B}\left(\sigma ; \sigma^{*}\right)$ is as above,

$$
\begin{aligned}
\left.\iota_{0, \sigma}\right|_{\overline{\mathcal{M}}_{1,\left(I_{P}(\sigma), J_{P}(\sigma)\right)}^{*} \times \overline{\mathfrak{M}}_{0, \varrho} T \overline{\mathfrak{M}}_{1, \sigma^{*}}^{0} / T\left(\overline{\mathcal{M}}_{1,\left(I_{P}(\sigma), J_{P}(\sigma)\right)}^{0} \times \overline{\mathfrak{M}}_{0, \varrho}\right)} \\
=\bigoplus_{i \in \aleph_{P}(\varrho)} \pi_{P}^{*} L_{i} \otimes \pi_{B}^{*} \pi_{i}^{*} L_{0},
\end{aligned}
$$

where $\aleph_{P}(\varrho) \subset \aleph_{B}(\sigma)$ is as in Section 3.3.

We note that for every $\sigma^{*} \in \mathcal{A}_{1}(d, k)$,

$$
\mathcal{A}_{1}\left(I_{P}\left(\sigma^{*}\right), J_{P}\left(\sigma^{*}\right)\right)=\bigsqcup_{\sigma \prec \sigma^{*}} \mathcal{A}_{P}\left(\sigma^{*} ; \sigma\right)
$$

Furthermore, if $\sigma_{1}, \sigma_{2} \in \mathcal{A}_{1}(d, k)$ are such that $\sigma_{1}, \sigma_{2} \prec \sigma^{*}$, then

$$
\rho_{1} \in \mathcal{A}_{P}\left(\sigma^{*} ; \sigma_{1}\right), \quad \rho_{2} \in \mathcal{A}_{P}\left(\sigma^{*} ; \sigma_{2}\right), \quad \rho_{1} \prec \rho_{2} \quad \Longrightarrow \quad \sigma_{1} \prec \sigma_{2} .
$$


Thus, we can choose an ordering $<$ on $\mathcal{A}_{1}\left(I_{P}\left(\sigma^{*}\right), J_{P}\left(\sigma^{*}\right)\right)$ extending the partial ordering $\prec$ of Section 2.3 such that

$$
\sigma_{1}<\sigma_{2}, \quad \rho_{1} \in \mathcal{A}_{P}\left(\sigma^{*} ; \sigma_{1}\right), \quad \rho_{2} \in \mathcal{A}_{P}\left(\sigma^{*} ; \sigma_{2}\right) \quad \Longrightarrow \quad \rho_{1}<\rho_{2},
$$

whenever $\sigma_{1}, \sigma_{2} \in \mathcal{A}_{1}(d, k)$ are such that $\sigma_{1}, \sigma_{2} \prec \sigma^{*}$. In the next subsection, we will refer to the blowup construction of Section 2.3 corresponding to such an ordering.

Similarly, if $\sigma^{\prime} \in \mathcal{A}_{1}(d, k)$,

$$
\mathcal{A}_{0}\left(\aleph_{B}\left(\sigma^{\prime}\right) ; d, J_{B}\left(\sigma^{\prime}\right)\right)=\bigsqcup_{\sigma^{\prime} \prec \sigma} \mathcal{A}_{B}\left(\sigma^{\prime} ; \sigma\right) .
$$

Furthermore, if $\sigma_{1}, \sigma_{2} \in \mathcal{A}_{1}(d, k)$ are such that $\sigma^{\prime} \prec \sigma_{1}, \sigma_{2}$, then

$$
\varrho_{1} \in \mathcal{A}_{B}\left(\sigma^{\prime} ; \sigma_{1}\right), \quad \varrho_{2} \in \mathcal{A}_{B}\left(\sigma^{\prime} ; \sigma_{2}\right), \quad \varrho_{1} \prec \varrho_{2} \quad \Longrightarrow \quad \sigma_{1} \prec \sigma_{2} .
$$

Thus, we can choose an ordering $<$ on $\mathcal{A}_{0}\left(\aleph_{B}\left(\sigma^{\prime}\right) ; d, J_{B}\left(\sigma^{\prime}\right)\right)$ extending the partial ordering $\prec$ of Section 3.3 such that

$$
\sigma_{1}<\sigma_{2}, \quad \varrho_{1} \in \mathcal{A}_{B}\left(\sigma^{\prime} ; \sigma_{1}\right), \quad \varrho_{2} \in \mathcal{A}_{B}\left(\sigma^{\prime} ; \sigma_{2}\right) \quad \Longrightarrow \quad \varrho_{1}<\varrho_{2}
$$

whenever $\sigma_{1}, \sigma_{2} \in \mathcal{A}_{1}(d, k)$ are such that $\sigma^{\prime} \prec \sigma_{1}, \sigma_{2}$. In the next subsection, we will refer to the blowup construction of Section 3.4 corresponding to such an ordering.

We denote by the main component $\overline{\mathfrak{M}}_{1, k}^{0}\left(\mathbb{P}^{n}, d\right)$ of the moduli space $\overline{\mathfrak{M}}_{1, k}\left(\mathbb{P}^{n}, d\right)$ by $\overline{\mathfrak{M}}_{1,(0)}^{0}$. If $\sigma \in \mathcal{A}_{1}(d, k)$, we put

$$
\begin{aligned}
& \overline{\mathcal{Z}}_{\sigma}^{0}=\iota_{0, \sigma}^{-1}\left(\overline{\mathfrak{M}}_{1,(0)}^{0}\right) \equiv \iota_{0, \sigma}^{-1}\left(\overline{\mathfrak{M}}_{1,(0)}^{0} \cap \overline{\mathfrak{M}}_{1, \sigma}^{0}\right) ; \\
& \mathcal{Z}_{\sigma}^{0}=\iota_{0, \sigma}^{-1}\left(\overline{\mathfrak{M}}_{1,(0)}^{0} \cap \mathfrak{M}_{1, \sigma}\right) \subset \overline{\mathcal{Z}}_{\sigma}^{0}, \quad \text { where } \quad \mathfrak{M}_{1, \sigma}=\mathfrak{M}_{1, \sigma}\left(\mathbb{P}^{n}, d\right) .
\end{aligned}
$$

We denote by $\mathcal{N} \overline{\mathcal{Z}}_{\sigma}^{0} \subset \mathcal{N}_{\iota_{0, \sigma}}^{\text {ide }}$ the normal cone $\mathcal{N}_{\iota_{0, \sigma}} \mid \overline{\mathfrak{M}}_{1,(0)}^{0}$ for $\left.\iota_{0, \sigma}\right|_{\overline{\mathcal{Z}}_{\sigma}^{0}}$ in $\overline{\mathfrak{M}}_{1,(0)}^{0}$. Its structure is described in Lemma 4.10 below. Let

$$
\mathcal{D}_{0, \sigma} \in \Gamma\left(\overline{\mathcal{M}}_{1,\left(I_{P}(\sigma), J_{P}(\sigma)\right)}^{0} \times \overline{\mathfrak{M}}_{0,\left(\aleph_{B}(\sigma), J_{B}(\sigma)\right)} ; \operatorname{Hom}\left(\mathcal{N}_{\iota_{0, \sigma}}^{\text {ide }}, \pi_{P}^{*} \mathbb{E}_{0}^{*} \otimes \pi_{B}^{*} \operatorname{ev}_{0}^{*} T \mathbb{P}^{n}\right)\right)
$$

be the section defined by

$$
\left.\mathcal{D}_{0, \sigma}\right|_{\pi_{P}^{*} L_{i} \otimes \pi_{B}^{*} \pi_{i}^{*} L_{0}}=\pi_{P}^{*} s_{0, i} \otimes \pi_{B}^{*} \pi_{i}^{*} \mathcal{D}_{0}, \quad \forall i \in[m],
$$

where $s_{0, i}$ and $\mathcal{D}_{0}$ are as in Sections 2.3 and 3.2, respectively.

Lemma 4.10 For all $\sigma \in \mathcal{A}_{1}(d, k), \overline{\mathfrak{M}}_{1,(0)}^{0}$ is a proper subvariety of $\overline{\mathfrak{M}}_{1, k}^{0}$ relative to the immersions $\iota_{0, \sigma}$ and $\bar{\iota}_{0, \sigma}$. Furthermore,

$$
\begin{aligned}
\mathcal{Z}_{\sigma}^{0} & =\left\{b \in \mathcal{M}_{1, I_{P}(\sigma) \sqcup J_{P}(\sigma)} \times \mathfrak{M}_{0,\left(\aleph_{B}(\sigma), J_{B}(\sigma)\right)}:\left.\operatorname{ker} \mathcal{D}_{0, \sigma}\right|_{b} \neq\{0\}\right\} \\
\left.\mathcal{N} \overline{\mathcal{Z}}_{\sigma}^{0}\right|_{\mathcal{Z}_{\sigma}^{0}} & =\left.\operatorname{ker} \mathcal{D}_{0, \sigma}\right|_{\mathcal{Z}_{\sigma}^{0}} .
\end{aligned}
$$


Finally, $\overline{\mathcal{Z}}_{\sigma}^{0}$ is the closure of $\mathcal{Z}_{\sigma}^{0}$ in $\overline{\mathcal{M}}_{1,\left(I_{P}(\sigma), J_{P}(\sigma)\right)}^{0} \times \overline{\mathfrak{M}}_{0,\left(\aleph_{B}(\sigma), J_{B}(\sigma)\right)}$ and $\mathcal{N} \overline{\mathcal{Z}}_{\sigma}^{0}$ is the closure of $\left.\mathcal{N} \overline{\mathcal{Z}}_{\sigma}^{0}\right|_{\mathcal{Z}_{\sigma}^{0}}$ in $\mathcal{N}_{\iota_{0, \sigma}}^{\text {ide }}$.

This lemma is a consequence of [24, Theorem 2.3] and related results. In particular, the first claim in the second sentence of Lemma 4.10 is a special case of the first statement of [24, Theorem 2.3]. The second claim is nearly a special case of the last statement of [24, Theorem 2.3], but some additional comments are required. Theorem 2.3 in [24] by itself is a purely topological statement, as it describes the topological structure of a neighborhood of each stratum of $\iota_{0, \sigma}\left(\overline{\mathcal{Z}}_{\sigma}^{0}\right)$ in $\overline{\mathfrak{M}}_{1,(0)}^{0}$. On the other hand, by [22, Subsection 4.1], $\left.\mathcal{N} \overline{\mathcal{Z}}_{\sigma}^{0}\right|_{\mathcal{Z}_{\sigma}^{0}}$ is contained in $\operatorname{ker} \mathcal{D}_{0, \sigma}$. The second claim in the second sentence of Lemma 4.10 can then be obtained from a dimension count and a comparison of the gluing construction used in the proof of [24, Theorem 2.3] with the analysis of limiting behavior in [22, Subsection 4.1]. This comparison implies that the gluing parameter in the analytic construction of [24] agrees to the first two orders in the zero limit with the smoothing parameter in algebraic geometry. Thus, $\left.\mathcal{N} \overline{\mathcal{Z}}_{\sigma}^{0}\right|_{\mathcal{Z}_{\sigma}^{0}}$ must be equal to $\operatorname{ker} \mathcal{D}_{\sigma, 0}$. These considerations also imply the first claim of Lemma 4.10. Alternatively, suppose that $d \leq n$. If the moduli space $\overline{\mathfrak{M}}_{1, \sigma}^{0}$ is nonempty, then $m \leq n$ and thus for a Zariski open subset $\mathcal{Z}_{\sigma ; 1}$ of $\mathcal{Z}_{\sigma}^{0}$

$$
\begin{gathered}
1 \leq\left.\operatorname{dim} \mathcal{N} \overline{\mathcal{Z}}_{\sigma}^{0}\right|_{\mathcal{Z}_{\sigma ; 1}}=1=\left.\operatorname{dim} \operatorname{ker} \mathcal{D}_{0, \sigma}\right|_{\mathcal{Z}_{\sigma ; 1}} \\
\left.\Longrightarrow \mathcal{N} \overline{\mathcal{Z}}_{\sigma}^{0}\right|_{\mathcal{Z}_{\sigma ; 1}}=\left.\operatorname{ker} \mathcal{D}_{0, \sigma}\right|_{\mathcal{Z}_{\sigma ; 1}}
\end{gathered}
$$

Since $\mathcal{D}_{0, \sigma}$ is transverse to the zero set over $\mathcal{Z}_{\sigma}^{0}$, the second claim in the second sentence of the lemma follows from (4-4), if $d \leq n$. The general case follows from the observation that

$$
\overline{\mathfrak{M}}_{1, \sigma}\left(\mathbb{P}^{n}, d\right)=\left\{[\Sigma, u] \in \overline{\mathfrak{M}}_{1, \sigma}\left(\mathbb{P}^{n+d}, d\right): u(\Sigma) \subset \mathbb{P}^{n}\right\}
$$

and the $d \leq n$ case.

The first claim in the last sentence of Lemma 4.10 can be obtained by combining the first statement of [24, Theorem 2.3], the $m=1$ case of [21, Theorem 2.8], and the Implicit Function Theorem. It also follows immediately from the last claim of Lemma 4.10. The latter can be deduced from [24, Theorem 2.3] as follows. Suppose first that $m \leq n$. In this case, [21, Theorem 2.8] implies that $\overline{\mathcal{Z}}_{\sigma}^{0}$ admits a stratification

$$
\overline{\mathcal{Z}}_{\sigma}^{0}=\mathcal{Z}_{\sigma ; 1} \sqcup \bigsqcup_{\alpha \in \mathcal{A}} \mathcal{Z}_{\sigma ; \alpha}
$$


such that $\mathcal{Z}_{\sigma ; 1}$ is a Zariski open subset of $\overline{\mathcal{Z}}_{\sigma}^{0}$,

$$
\begin{gathered}
\mathcal{Z}_{\sigma ; 1} \subset \mathcal{Z}_{\sigma}^{0},\left.\quad \operatorname{dim} \mathcal{N} \overline{\mathcal{Z}}_{\sigma}^{0}\right|_{b}=1 \forall b \in \mathcal{Z}_{\sigma ; 1}, \\
\max \left\{\left.\operatorname{dim} \mathcal{N} \overline{\mathcal{Z}}_{\sigma}^{0}\right|_{b}: b \in \mathcal{Z}_{\sigma ; \alpha}\right\} \leq \operatorname{codim}_{\overline{\mathcal{Z}}_{\sigma}^{0}} \mathcal{Z}_{\sigma ; \alpha} \forall \alpha \in \mathcal{A} ;
\end{gathered}
$$

see the next paragraph. Let

$$
\widetilde{\mathcal{Z}}_{\sigma}^{0}=\left.\mathbb{P} \mathcal{N} \overline{\mathcal{Z}}_{\sigma}^{0} \subset \mathbb{P} \mathcal{N}_{\iota_{0, \sigma}}^{\text {ide }}\right|_{\overline{\mathcal{Z}}_{\sigma}^{0}}
$$

be the exceptional divisor for the blowup of $\overline{\mathfrak{M}}_{1,(0)}^{0}{\widetilde{Z^{\prime}}}_{\sigma}^{\text {along }} \overline{\mathfrak{M}}_{1, \sigma}^{0}$. Since all irreducible components of $\widetilde{\mathcal{Z}}_{\sigma}^{0}$ must be of the same dimension, $\widetilde{\mathcal{Z}}_{\sigma}^{0}$ must be the closure of $\left.\widetilde{\mathcal{Z}}_{\sigma}^{0}\right|_{\mathcal{Z}_{\sigma}^{0}}$ by (4-5). This closure property remains valid even if we do not assume that $m \leq n$ for the following reason. Let $\mathrm{pt} \in \mathbb{P}^{n+d}$ be any point not contained in $\mathbb{P}^{n}$. Let

$$
\pi: \mathbb{P}^{n+d}-\{\mathrm{pt}\} \longrightarrow \mathbb{P}^{\mathrm{n}}
$$

be the corresponding linear projection. It induces projection maps

$$
\begin{aligned}
& \varphi:\left\{[\Sigma, u] \in \overline{\mathfrak{M}}_{1, k}^{0}\left(\mathbb{P}^{n+d}, d\right): \operatorname{pt} \notin u(\Sigma)\right\} \longrightarrow \overline{\mathfrak{M}}_{1, k}^{0}\left(\mathbb{P}^{n}, d\right), \\
& \tilde{\varphi}:\left\{[\Sigma, u ; v] \in \widetilde{\mathcal{Z}}_{\sigma}^{0}\left(\mathbb{P}^{n+d}, d\right): \operatorname{pt} \notin u(\Sigma)\right\} \longrightarrow \widetilde{\mathcal{Z}}_{\sigma}^{0}\left(\mathbb{P}^{n}, d\right) .
\end{aligned}
$$

The latter map takes $\left.\widetilde{\mathcal{Z}}_{\sigma}^{0}\left(\mathbb{P}^{n+d}, d\right)\right|_{\mathcal{Z}_{\sigma}^{0}\left(\mathbb{P}^{n+d}, d\right)}$ to $\left.\widetilde{\mathcal{Z}}_{\sigma}^{0}\left(\mathbb{P}^{n}, d\right)\right|_{\mathcal{Z}_{\sigma}^{0}\left(\mathbb{P}^{n}, d\right)}$. Since the closure of

$$
\left.\widetilde{\mathcal{Z}}_{\sigma}^{0}\left(\mathbb{P}^{n+d}, d\right)\right|_{\mathcal{Z}_{\sigma}^{0}\left(\mathbb{P}^{n+d}, d\right)}
$$

contains $\widetilde{\mathcal{Z}}_{\sigma}^{0}\left(\mathbb{P}^{n}, d\right)$, it follows that so does the closure of $\left.\widetilde{\mathcal{Z}}_{\sigma}^{0}\left(\mathbb{P}^{n}, d\right)\right|_{\mathcal{Z}_{\sigma}^{0}\left(\mathbb{P}^{n}, d\right)}$. This observation implies the last claim of Lemma 4.10.

We conclude this subsection by briefly describing the stratification mentioned above. A stratum $\mathfrak{M}_{\Gamma_{B}}$ of $\overline{\mathfrak{M}}_{0,\left(\aleph_{B}(\sigma), J_{B}(\sigma)\right)}$ corresponds to a tuple $\Gamma_{B} \equiv\left(\Gamma_{B ; l}\right)_{l \in \aleph_{B}(\sigma)}$ of dual graphs, all of which are trees. The vertices of $\Gamma_{B ; l}$ correspond to the irreducible components of the domain of the stable map $b_{l}$ in the definition of $\overline{\mathfrak{M}}_{0,\left(\aleph_{B}(\sigma), J_{B}(\sigma)\right)}$ at the beginning of Section 3.3. Each vertex $v$ of $\Gamma_{B ; l}$ is labeled by a nonnegative integer, which specifies the degree of the stable map $b_{l}$ on the corresponding component $\Sigma_{v}$. There is an edge in $\Gamma_{B ; l}$ between two vertices if and only if the two corresponding components of the domain share a node. In addition, there are tails attached at some vertices of $\Gamma_{B ; l}$, which are labeled by the indexing set for marked points of the map $b_{l}$, ie $J_{l, P}$ in the notation of Section 3.3. Let $v_{l}^{*}$ be the vertex of $\Gamma_{B ; l}$ to which the tail corresponding to the marked point 0 is attached. If the degree of $v_{l}^{*}$ is positive, let

$$
\chi_{l}\left(\Gamma_{B}\right) \equiv \chi_{l}\left(\Gamma_{B ; l}\right)=\left\{v_{l}^{*}\right\} .
$$


Otherwise, denote by $\chi_{l}\left(\Gamma_{B}\right)$ the set of positive-degree vertices of $\Gamma_{B ; l}$ that are not separated from $v_{l}^{*}$ by a positive-degree vertex. Suppose

$$
b \equiv\left(b_{l}\right)_{l \in \aleph_{B}(\sigma)} \in \mathfrak{M}_{\Gamma_{B}} \equiv \overline{\mathfrak{M}}_{0,\left(\aleph_{B}(\sigma), J_{B}(\sigma)\right)} \cap \prod_{l \in \boldsymbol{\aleph}_{B}(\sigma)} \mathfrak{M}_{\Gamma_{B ; l}}, \quad \text { with } \quad b_{l}=\left[\Sigma_{l}, u_{l}\right]
$$

as in the paragraph preceding Lemma 3.8. If $l \in \aleph_{B}(\sigma)$ and $v=v_{l}^{*}$, let

$$
\left.\operatorname{Im} \mathcal{D}_{v}\right|_{b}=\left.\left.\operatorname{Im} \mathcal{D}_{0}\right|_{b_{l}} \equiv \operatorname{Im} d u_{l}\right|_{x_{0}\left(b_{l}\right)} \subset T_{\mathrm{ev}_{0}(b)} \mathbb{P}^{n} .
$$

If $v$ is a vertex of $\Gamma_{B ; l}$ different from $v_{l}^{*}$, we denote by $\left.\operatorname{Im} \mathcal{D}_{v}\right|_{b}$ the image of $d\left\{\left.u_{l}\right|_{\Sigma_{v}}\right\}$ at the node of $\Sigma_{v}$ corresponding to the edge of $\Gamma_{B ; l}$ that leaves $v$ on the unique path from $v$ on $v_{l}^{*}$ in $\Gamma_{B ; l}$. Note that if $v \in \chi_{l}\left(\Gamma_{B}\right)$, the image of this node under $u_{l}$ is $\operatorname{ev}_{0}(b)$. We set

$$
\chi(\Gamma)=\bigsqcup_{l \in \aleph_{B}(\sigma)} \chi_{l}\left(\Gamma_{B}\right)
$$

With $b$ as above, let

$$
\left.\operatorname{codim} \mathcal{D}\right|_{b}=\left|\chi\left(\Gamma_{B}\right)\right|-\operatorname{dim} \operatorname{Span}\left\{\left.\operatorname{Im} \mathcal{D}_{v}\right|_{b}: v \in \chi_{l}\left(\Gamma_{B}\right), l \in \boldsymbol{\aleph}_{B}(\sigma)\right\} .
$$

For each pair $\alpha=\left(\Gamma_{B}, \mu\right)$, where $\mu \in \mathbb{Z}^{+}$is such that

$$
\max \left(1,\left|\chi\left(\Gamma_{B}\right)\right|-n\right) \leq \mu \leq\left|\chi\left(\Gamma_{B}\right)\right|,
$$

we put

$$
\mathcal{Z}_{\Gamma_{B} ; \alpha}=\left\{b \in \mathfrak{M}_{\Gamma_{B}}:\left.\operatorname{codim} \mathcal{D}\right|_{b}=\mu\right\} .
$$

By the first statement of [24, Theorem 2.3],

$$
\overline{\mathcal{Z}}_{\sigma}^{0}=\bigsqcup_{\alpha} \mathcal{Z}_{\sigma ; \alpha}, \quad \text { where } \quad \mathcal{Z}_{\sigma ; \alpha}=\overline{\mathcal{M}}_{1, I_{P}(\sigma) \sqcup J_{P}(\sigma)} \times \mathcal{Z}_{\Gamma_{B} ; \alpha}
$$

The disjoint union is taken over all pairs $\alpha=(\Gamma, \mu)$ as described above. From transversality as in the first claim of Lemma 3.8, it is easy to see that

$$
\begin{aligned}
\operatorname{codim}_{\mathfrak{M}_{\Gamma_{B}}} \mathcal{Z}_{\Gamma_{B} ; \alpha} & =\left(n-\left(\left|\chi\left(\Gamma_{B}\right)\right|-\mu\right)\right) \mu \\
& \geq n-\left(\left|\chi\left(\Gamma_{B}\right)\right|-\mu\right) ;
\end{aligned}
$$

see the end of [25, Subsection 2.3], for example. The above inequality follows from the first inequality in (4-6). By (4-7), if $m=\left|\aleph_{B}(\sigma)\right| \leq n$,

$$
\begin{aligned}
& \operatorname{codim}_{\overline{\mathcal{Z}}_{\sigma}^{0}} \mathcal{Z}_{\sigma ; \alpha}= \operatorname{codim}_{\mathfrak{M}_{\Gamma_{B}}} \mathcal{Z}_{\Gamma_{B} ; \alpha}+\operatorname{codim}_{\overline{\mathfrak{M}}_{0,\left(\aleph_{B}(\sigma), J_{B}(\sigma)\right)}} \mathfrak{M}_{\Gamma_{B}} \\
&-\operatorname{codim}_{\overline{\mathcal{M}}_{1, I_{P}(\sigma) \sqcup J_{P}(\sigma)} \times \overline{\mathfrak{M}}_{0,\left(\aleph_{B}(\sigma), J_{B}(\sigma)\right)}} \overline{\mathcal{Z}}_{\sigma}^{0} \\
& \geq\left(n-\left|\chi\left(\Gamma_{B}\right)\right|+\mu\right)+\left(\left|\chi\left(\Gamma_{B}\right)\right|-\left|\aleph_{B}(\sigma)\right|\right)-\left(n-\left|\aleph_{B}(\sigma)\right|+1\right)=\mu-1 .
\end{aligned}
$$


On the other hand, by the last statement of [24, Theorem 2.3],

$$
\max \left\{\left.\operatorname{dim} \mathcal{N} \overline{\mathcal{Z}}_{\sigma}^{0}\right|_{b}: b \in \mathcal{Z}_{\sigma ; \alpha}\right\}=\mu \text {. }
$$

We conclude that

$$
\max \left\{\left.\operatorname{dim} \mathcal{N} \overline{\mathcal{Z}}_{\sigma}^{0}\right|_{b}: b \in \mathcal{Z}_{\sigma ; \alpha}\right\} \leq \operatorname{codim}_{\overline{\mathcal{Z}}_{\sigma}^{0}} \mathcal{Z}_{\sigma ; \alpha}+1 .
$$

The equality holds if and only if $\mu=1$ and $\Gamma_{B}$ is a tuple of one-vertex graphs, ie the image of $\mathcal{M}_{1, I_{P}(\sigma) \sqcup J_{P}(\sigma)} \times \mathfrak{M}_{\Gamma_{B}}$ under $\iota_{0, \sigma}$ is contained in $\mathfrak{M}_{1, \sigma}$. This observation concludes the proof of the stratification claim made in the previous paragraph.

\subsection{Inductive construction}

This subsection is the analogue of Section 3.4 in the present situation. Suppose $\sigma \in$ $\mathcal{A}_{1}(d, k)$ and we have constructed

(I1) an idealized blowup $\pi_{\sigma-1}: \overline{\mathfrak{M}}_{1, k}^{\sigma-1} \longrightarrow \overline{\mathfrak{M}}_{1, k}^{0}$ such that $\pi_{\sigma-1}$ is an isomorphism outside of the preimages of the subvarieties $\overline{\mathfrak{M}}_{1, \sigma^{\prime}}^{0}$ with $\sigma^{\prime} \leq \sigma-1$;

(I2) for each $\sigma^{\prime} \in\{(0)\} \sqcup \mathcal{A}_{1}(d, k)$, a subvariety $\overline{\mathfrak{M}}_{1, \sigma^{\prime}}^{\sigma-1}$ of $\overline{\mathfrak{M}}_{1, k}^{\sigma-1}$ such that

$$
\overline{\mathfrak{M}}_{1, k}^{\sigma-1}=\overline{\mathfrak{M}}_{1,(0)}^{\sigma-1} \cup \underset{\sigma^{\prime} \in \mathcal{A}_{1}(d, k)}{\bigcup} \overline{\mathfrak{M}}_{1, \sigma^{\prime}}^{\sigma-1}, \quad \pi_{\sigma-1}\left(\overline{\mathfrak{M}}_{1, \sigma^{\prime}}^{\sigma-1}\right)=\overline{\mathfrak{M}}_{1, \sigma^{\prime}}^{0} \forall \sigma^{\prime} \in\{(0)\} \sqcup \mathcal{A}_{1}(d, k),
$$

and $\overline{\mathfrak{M}}_{1, \sigma^{*}}^{\sigma-1}$ is the proper transform of $\overline{\mathfrak{M}}_{1, \sigma^{*}}^{0}$ for $\sigma^{*}=(0)$ and for all $\sigma^{*} \in$ $\mathcal{A}_{1}(d, k)$ such that $\sigma^{*}>\sigma-1$.

We assume that

(I3) for all $\sigma_{1}, \sigma_{2} \in \mathcal{A}_{1}(d, k)$ such that $\sigma_{1} \neq \sigma_{2}, \sigma_{1} \nprec \sigma_{2}, \sigma_{2} \nprec \sigma_{1}$, and $\sigma-1<\sigma_{1}, \sigma_{2}$,

$$
\overline{\mathfrak{M}}_{1, \sigma_{1}}^{\sigma-1} \cap \overline{\mathfrak{M}}_{1, \sigma_{2}}^{\sigma-1} \begin{cases}\subset \overline{\mathfrak{M}}_{1, \widetilde{\sigma}\left(\sigma_{1}, \sigma_{2}\right)}^{\sigma-1}, & \text { if } \tilde{\sigma}\left(\sigma_{1}, \sigma_{2}\right)>\sigma-1 ; \\ =\varnothing, & \text { otherwise, }\end{cases}
$$

where $\tilde{\sigma}\left(\sigma_{1}, \sigma_{2}\right)$ is as in Lemma 4.6.

We also assume that for every $\sigma^{\prime} \in A_{1}(d, k)$ such that $\sigma^{\prime} \leq \sigma-1$ :

(I4) $\overline{\mathfrak{M}}_{1, \sigma^{\prime}}^{\sigma-1}$ is the image of a $G_{\sigma^{\prime}}$-invariant immersion

$$
\iota_{\sigma-1, \sigma^{\prime}}: \widetilde{\mathcal{M}}_{1,\left(I_{P}\left(\sigma^{\prime}\right), J_{P}\left(\sigma^{\prime}\right)\right)} \times \widetilde{\mathfrak{M}}_{0,\left(\aleph_{B}\left(\sigma^{\prime}\right), J_{B}\left(\sigma^{\prime}\right)\right)}^{\varrho_{\sigma^{\prime}}(\sigma-1)} \longrightarrow \overline{\mathfrak{M}}_{1, k}^{\sigma-1},
$$

where $\varrho_{\sigma^{\prime}}(\sigma-1)= \begin{cases}\max \left\{\varrho \in \mathcal{A}_{B}\left(\sigma^{\prime} ; \sigma^{*}\right): \sigma^{\prime} \prec \sigma^{*} \leq \sigma-1\right\}, & \text { if } \exists \sigma^{*} \in \mathcal{A}_{1}(d, k) \\ 0, & \text { s.t. } \sigma^{\prime} \prec \sigma^{*} \leq \sigma-1 ;\end{cases}$ 
and

$$
\widetilde{\mathfrak{M}}_{0,\left(\aleph_{B}\left(\sigma^{\prime}\right), J_{B}\left(\sigma^{\prime}\right)\right)}^{\varrho_{\sigma^{\prime}}(\sigma-1)} \equiv \widetilde{\mathfrak{M}}_{0,\left(\aleph_{B}\left(\sigma^{\prime}\right), J_{B}\left(\sigma^{\prime}\right)\right)}^{\varrho_{\sigma^{\prime}}(\sigma-1)}\left(\mathbb{P}^{n}, d\right)
$$

is the blowup of $\widetilde{\mathfrak{M}}_{0,\left(\aleph_{B}\left(\sigma^{\prime}\right), J_{B}\left(\sigma^{\prime}\right)\right)}^{0}\left(\mathbb{P}^{n}, d\right)$ constructed in Section 3.4;

(I5) if $\sigma^{*} \in \mathcal{A}_{1}(d, k)$ is such that $\sigma-1<\sigma^{*}$ and $\sigma^{\prime} \prec \sigma^{*}$, then

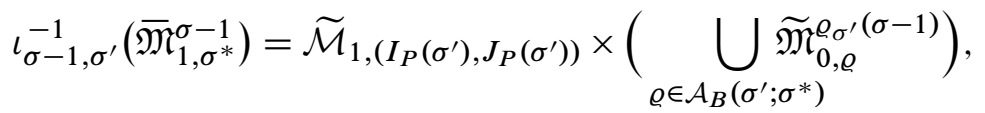

where $\widetilde{\mathfrak{M}}_{0, \varrho}^{\varrho_{\sigma^{\prime}}(\sigma-1)} \equiv \widetilde{\mathfrak{M}}_{0, \varrho}^{\varrho \sigma^{\prime}}(\sigma-1)\left(\mathbb{P}^{n}, d\right)$ is the subvariety of $\widetilde{\mathfrak{M}}_{\left.0, \boldsymbol{\aleph}_{B}^{\prime}\left(\sigma^{\prime}\right), J_{B}\left(\sigma^{\prime}\right)\right)}^{\varrho_{\sigma^{\prime}}(\sigma-1)}$ described in Section 3.4;

(I6) an idealized normal bundle for the immersion $\iota_{\sigma-1, \sigma^{\prime}}$ is given by

$$
\mathcal{N}_{\iota_{\sigma-1, \sigma^{\prime}}}^{\text {ide }}=\pi_{P}^{*} \mathbb{L} \otimes \pi_{B}^{*} \pi_{\varrho_{\sigma^{\prime}}(\sigma-1)}^{*} \gamma\left(\aleph_{B}\left(\sigma^{\prime}\right), J_{B}\left(\sigma^{\prime}\right)\right),
$$

where $\pi_{B}, \pi_{P}: \widetilde{\mathcal{M}}_{1,\left(I_{P}\left(\sigma^{\prime}\right), J_{P}\left(\sigma^{\prime}\right)\right)} \times \widetilde{\mathfrak{M}}_{0,\left(\aleph_{B}\left(\sigma^{\prime}\right), J_{B}\left(\sigma^{\prime}\right)\right)}^{\varrho_{\sigma^{\prime}}(\sigma-1)}$

$$
\longrightarrow \widetilde{\mathcal{M}}_{1,\left(I_{P}\left(\sigma^{\prime}\right), J_{P}\left(\sigma^{\prime}\right)\right)}, \widetilde{\mathfrak{M}}_{0,\left(\aleph_{B}\left(\sigma^{\prime}\right), J_{B}\left(\sigma^{\prime}\right)\right)} \varrho_{\sigma^{\prime}}(\sigma-1)
$$

are the two projection maps and $\mathbb{L} \longrightarrow \widetilde{\mathcal{M}}_{1,\left(I_{P}\left(\sigma^{\prime}\right), J_{P}\left(\sigma^{\prime}\right)\right)}$ is the universal tangent line bundle of Section 2.3;

(I7) $\overline{\mathcal{Z}}_{\sigma^{\prime}}^{\sigma-1} \equiv \iota_{\sigma-1, \sigma^{\prime}}^{-1}\left(\overline{\mathfrak{M}}_{1,(0)}^{\sigma-1}\right)$ is the closure of

$$
\mathcal{Z}_{\sigma^{\prime}}^{\sigma-1} \equiv \widetilde{\mathcal{M}}_{1,\left(I_{P}\left(\sigma^{\prime}\right), J_{P}\left(\sigma^{\prime}\right)\right)} \times(\widetilde{\mathcal{D}}_{\varrho_{\sigma^{\prime}}(\sigma-1)}^{-1}(0)-\underbrace{}_{\substack{\varrho \in \mathcal{A}_{B}\left(\aleph_{B}\left(\sigma^{\prime}\right) ; d, J_{B}\left(\sigma^{\prime}\right)\right) \\ \varrho_{\sigma^{\prime}}(\sigma-1)<\varrho}} \widetilde{\mathfrak{M}}_{0, \varrho}^{\varrho_{\sigma^{\prime}}(\sigma-1)})
$$

in $\widetilde{\mathcal{M}}_{1,\left(I_{P}\left(\sigma^{\prime}\right), J_{P}\left(\sigma^{\prime}\right)\right)} \times \widetilde{\mathfrak{M}}_{0,\left(\aleph_{B}\left(\sigma^{\prime}\right), J_{B}\left(\sigma^{\prime}\right)\right)}^{\varrho_{\sigma^{\prime}}(\sigma-1)}$ and

$$
\mathcal{N} \overline{\mathcal{Z}}_{\sigma^{\prime}}^{\sigma-1} \equiv \mathcal{N}_{\iota_{\sigma-1, \sigma^{\prime}} \mid \overline{\mathfrak{M}}_{1,(0)}^{\sigma-1}}=\left.\mathcal{N}_{\iota_{\sigma-1, \sigma^{\prime}}}^{\text {ide }}\right|_{\overline{\mathcal{Z}}_{\sigma^{\prime}}^{\sigma-1}}
$$

is the normal cone for $\iota_{\sigma-1, \sigma^{\prime}} \mid \overline{\mathfrak{M}}_{1,(0)}^{\sigma-1}$ in $\overline{\mathfrak{M}}_{1, k}^{\sigma-1}$;

(I8) the immersion map

$$
\bar{\iota}_{\sigma-1, \sigma^{\prime}}:\left(\widetilde{\mathcal{M}}_{1,\left(I_{P}\left(\sigma^{\prime}\right) ; J_{P}\left(\sigma^{\prime}\right)\right)} \times \widetilde{\mathfrak{M}}_{0,\left(\aleph_{B}\left(\sigma^{\prime}\right), J_{B}\left(\sigma^{\prime}\right)\right)}^{\varrho_{\sigma^{\prime}}(\sigma-1)}\right) / G_{\sigma^{\prime}} \longrightarrow \overline{\mathfrak{M}}_{1, k}^{\sigma-1}
$$

induced by $\iota_{\sigma-1, \sigma^{\prime}}$ is an embedding.

Furthermore, we assume that for every $\sigma^{*} \in A_{1}(d, k)$ such that $\sigma^{*}>\sigma-1$ : 
(I9) the domain of the $G_{\sigma^{*}}$-invariant immersion $\iota_{\sigma-1, \sigma^{*}}$ induced by $\iota_{0, \sigma^{*}}$ is

$$
\overline{\mathcal{M}}_{1,\left(I_{P}\left(\sigma^{*}\right), J_{P}\left(\sigma^{*}\right)\right)}^{\rho_{\sigma^{*}}(\sigma-1)} \times \overline{\mathfrak{M}}_{0,\left(\aleph_{B}\left(\sigma^{*}\right), J_{B}\left(\sigma^{*}\right)\right)}
$$

where $\quad \rho_{\sigma^{*}}(\sigma-1)=\left\{\begin{array}{cl}\max \left\{\rho \in \mathcal{A}_{P}\left(\sigma^{*} ; \sigma^{\prime}\right):\right. & \text { if } \exists \sigma^{\prime} \in \mathcal{A}_{1}(d, k) \\ \left.\sigma^{\prime} \leq \sigma-1, \sigma^{\prime} \prec \sigma^{*}\right\}, & \text { s.t. } \sigma^{\prime} \leq \sigma-1, \sigma^{\prime} \prec \sigma^{*} ; \\ 0, & \text { otherwise, }\end{array}\right.$

and $\overline{\mathcal{M}}_{1,\left(I_{P}\left(\sigma^{*}\right), J_{P}\left(\sigma^{*}\right)\right)}^{\rho_{\sigma^{*}}(\sigma-1)} \longrightarrow \overline{\mathcal{M}}_{1, I_{P}\left(\sigma^{*}\right) \sqcup J_{P}\left(\sigma^{*}\right)}$ is the blowup constructed in Section 2.3;

(I10) if $\sigma^{\prime} \in \mathcal{A}_{1}(d, k)$ is such that $\sigma-1<\sigma^{\prime} \prec \sigma^{*}$, then

$$
\iota_{\sigma-1, \sigma^{*}}^{-1}\left(\widetilde{\mathfrak{M}}_{1, \sigma^{\prime}}^{\sigma-1}\right)=\left(\bigcup_{\rho \in \mathcal{A}_{P}\left(\sigma^{*} ; \sigma^{\prime}\right)} \overline{\mathcal{M}}_{1, \rho}^{\rho_{\sigma^{*}}(\sigma-1)}\right) \times \overline{\mathfrak{M}}_{0,\left(\aleph_{B}\left(\sigma^{*}\right), J_{B}\left(\sigma^{*}\right)\right)} ;
$$

(I11) if $\sigma^{*}$ is as in Lemma 4.7, an idealized normal bundle for the immersion $\iota_{\sigma-1, \sigma^{*}}$ is given by

$$
\mathcal{N}_{l_{\sigma-1, \sigma^{*}}}^{\mathrm{ide}}=\bigoplus_{i \in\left[m^{*}\right]} \pi_{P}^{*} L_{\rho_{\sigma^{*}}(\sigma-1), i} \otimes \pi_{B}^{*} \pi_{i}^{*} L_{0}
$$

where $\pi_{P}, \pi_{B}: \overline{\mathcal{M}}_{1,\left(I_{P}\left(\sigma^{*}\right), J_{P}\left(\sigma^{*}\right)\right)}^{\rho_{\sigma}^{*}(\sigma-1)} \times \overline{\mathfrak{M}}_{0,\left(\aleph_{B}\left(\sigma^{*}\right), J_{B}\left(\sigma^{*}\right)\right)}$

$$
\longrightarrow \overline{\mathcal{M}}_{1,\left(I_{P}\left(\sigma^{*}\right), J_{P}\left(\sigma^{*}\right)\right)}^{\rho_{\sigma^{*}}(\sigma-1)}, \overline{\mathfrak{M}}_{0,\left(\aleph_{B}\left(\sigma^{*}\right), J_{B}\left(\sigma^{*}\right)\right)}
$$

are the two projection maps and $L_{\rho_{\sigma^{*}}(\sigma-1), i} \longrightarrow \overline{\mathcal{M}}_{1,\left(I_{P}\left(\sigma^{*}\right), J_{P}\left(\sigma^{*}\right)\right)}^{\rho_{\sigma^{*}}(\sigma-1)}$ is the line bundle constructed in Section 2.3;

(I12) $\overline{\mathcal{Z}}_{\sigma^{*}}^{\sigma-1} \equiv \iota_{\sigma-1, \sigma^{*}}^{-1}\left(\overline{\mathfrak{M}}_{1,(0)}^{\sigma-1}\right)$ is the closure of

$$
\mathcal{Z}_{\sigma^{*}}^{0} \subset \overline{\mathcal{M}}_{1,\left(I_{P}\left(\sigma^{*}\right), J_{P}\left(\sigma^{*}\right)\right)}^{\rho_{\sigma^{*}}(\sigma-1)} \times \overline{\mathfrak{M}}_{0,\left(\aleph_{B}\left(\sigma^{*}\right), J_{B}\left(\sigma^{*}\right)\right)}
$$

and the normal cone

$$
\mathcal{N} \overline{\mathcal{Z}}_{\sigma^{*}}^{\sigma-1} \equiv \mathcal{N}_{\iota_{\sigma-1, \sigma *} \mid \overline{\mathfrak{M}}_{1,(0)}^{\sigma-1}}
$$

for $\iota_{\sigma-1, \sigma^{*}} \mid \overline{\mathcal{Z}}_{\sigma^{*}}^{\sigma-1}$ is the closure of $\left.\mathcal{N} \overline{\mathcal{Z}}_{\sigma^{*}}^{0}\right|_{\mathcal{Z}_{\sigma^{*}}^{0}}$ in $\mathcal{N}_{\iota_{\sigma-1, \sigma^{*}}}^{\text {ide }}$;

(I13) $\operatorname{Im}^{s} \bar{\iota}_{\sigma-1, \sigma^{*}} \subset \bigcup_{\sigma-1<\sigma^{\prime}<\sigma^{*}} \widetilde{\mathfrak{M}}_{1, \sigma^{\prime}}^{\sigma-1}$, where

$$
\bar{\iota}_{\sigma-1, \sigma^{*}}:\left(\overline{\mathcal{M}}_{1,\left(I_{P}\left(\sigma^{*}\right), J_{P}\left(\sigma^{*}\right)\right)}^{\rho_{\sigma *}(\sigma-1)} \times \overline{\mathfrak{M}}_{0,\left(\aleph_{B}\left(\sigma^{*}\right), J_{B}\left(\sigma^{*}\right)\right)}\right) / G_{\sigma^{*}} \longrightarrow \overline{\mathfrak{M}}_{1, k}^{\sigma-1},
$$

is the immersion map induced by $\iota_{\sigma-1, \sigma^{*}}$. 
Finally, we assume that

(I14) the collections $\left\{\iota_{\sigma-1, \sigma^{\prime}}\right\}_{\sigma^{\prime} \in \mathcal{A}_{1}(d, k)}$ and $\left\{\bar{l}_{\sigma-1, \sigma^{\prime}}\right\}_{\sigma^{\prime} \in \mathcal{A}_{1}(d, k)}$ of immersions are properly self-intersecting;

(I15) for all $\sigma^{\prime} \in \mathcal{A}_{1}(d, k)$, the subvariety $\overline{\mathfrak{M}}_{1,(0)}^{\sigma-1}$ of $\overline{\mathfrak{M}}_{1, k}^{\sigma-1}$ is proper relative to the immersions $\iota_{\sigma-1, \sigma^{\prime}}$ and $\bar{\iota}_{\sigma-1, \sigma^{\prime}}$.

By the inductive assumption (I3), if $\sigma_{1}$ and $\sigma_{2}$ are noncomparable elements of $\left(\mathcal{A}_{1}(d, k), \prec\right)$, the proper transforms of $\overline{\mathfrak{M}}_{1, \sigma_{1}}^{0}$ and $\overline{\mathfrak{M}}_{1, \sigma_{2}}^{0}$ become disjoint by the time either is ready to be blown up for any ordering < extending the partial ordering $\prec$. Similarly to the three blowup constructions encountered previously, (I3) will imply that the end result of the present blowup construction is independent of the choice of an extension $<$. By (I9), our blowup construction modifies each immersion $\iota_{0, \sigma^{*}}$ by changing the first factor of the domain according to the blowup construction of Section 2.3, until a proper transform of the image of $\iota_{0, \sigma^{*}}$ is to be blown up; see below. By (I11) and (I13), in the process, the singular locus of $\iota_{0, \sigma^{*}}$ disappears and the first component in every summand of $\mathcal{N}_{\iota_{0, \sigma^{*}}}^{\text {ide }}$ gets twisted to $\mathbb{L}$. In particular, all blowup loci are smooth. On the other hand, by the inductive assumptions (I7) and (I8), for $\sigma^{\prime} \leq \sigma-1$ the intersection of the proper transform of $\overline{\mathfrak{M}}_{1,(0)}^{0}$ with the proper transform of the exceptional divisor $\overline{\mathfrak{M}}_{1, \sigma^{\prime}}^{\sigma^{\prime}}$ is an embedding of a subvariety of a smooth variety. The singular locus of this subvariety is annihilated by the time the entire blowup construction is complete, according to the inductive assumptions ( I7) above and the inductive assumption (I4) in Section 3.4. These assumptions imply that the proper transform of $\overline{\mathfrak{M}}_{1,(0)}^{0}$ after the final blowup step is smooth.

We note that all of the assumptions (I1)-(I15) are satisfied if $\sigma-1$ is replaced by 0 . In particular, (I3) is a restatement of Lemma 4.6, while (I10)-(I15) are contained in Lemmas 4.7, 4.8, and 4.10.

If $\sigma \in \mathcal{A}_{1}(d, k)$ is as above, let

$$
\tilde{\pi}_{\sigma}: \overline{\mathfrak{M}}_{1, k}^{\sigma} \longrightarrow \overline{\mathfrak{M}}_{1, k}^{\sigma-1}
$$

be the idealized blowup of $\overline{\mathfrak{M}}_{1, k}^{\sigma-1}$ along $\overline{\mathfrak{M}}_{1, \sigma}^{\sigma-1}$, which is a smooth subvariety by the inductive assumption (I13). We denote the idealized exceptional divisor,

$$
\mathcal{E}_{\overline{\mathfrak{M}}_{1, \sigma}^{\sigma-1}}^{\text {ide }}=\mathbb{P} \mathcal{N}_{\bar{\iota}_{\sigma-1, \sigma}}^{\text {ide }},
$$

by $\overline{\mathfrak{M}}_{1, \sigma}^{\sigma}$. For each $\sigma^{\prime} \in\{(0)\} \sqcup\left(\mathcal{A}_{1}(d, k)-\{\sigma\}\right)$, we denote by

$$
\overline{\mathfrak{M}}_{1, \sigma^{\prime}}^{\sigma} \subset \mathrm{B}_{\overline{\mathfrak{M}}_{1, \sigma}^{\sigma-1}} \overline{\mathfrak{M}}_{1, k}^{\sigma-1} \subset \overline{\mathfrak{M}}_{1, k}^{\sigma}
$$


the proper transform of $\overline{\mathfrak{M}}_{1, \sigma^{\prime}}^{\sigma-1}$. Let $\pi_{\sigma}=\pi_{\sigma-1} \circ \tilde{\pi}_{\sigma}$.

The inductive assumptions $(I 1)$ and (I2), with $\sigma-1$ replaced by $\sigma$, are clearly satisfied, while (I3), (I8) for $\sigma^{\prime} \neq \sigma$, and (I13)-(I15) follow from (2) of Lemma 3.3, Corollary 3.4, and Lemma 4.5. On the other hand, by (I9), the domain of the immersion $\iota_{\sigma-1, \sigma}$ is

$$
\overline{\mathcal{M}}_{1,\left(I_{P}(\sigma), J_{P}(\sigma)\right)}^{\rho_{\sigma}(\sigma-1)} \times \overline{\mathfrak{M}}_{0,\left(\aleph_{B}(\sigma), J_{B}(\sigma)\right)}=\widetilde{\mathcal{M}}_{1,\left(I_{P}(\sigma), J_{P}(\sigma)\right)} \times \overline{\mathfrak{M}}_{0,\left(\aleph_{B}(\sigma), J_{B}(\sigma)\right)} .
$$

By ( $I 11)$, the chosen idealized normal bundle for the immersion $\iota_{\sigma-1, \sigma}$ is given by

$$
\mathcal{N}_{l_{\sigma-1, \sigma}}^{\mathrm{ide}}=\bigoplus_{i \in[m]} \pi_{P}^{*} L_{\rho_{\sigma}(\sigma-1), i} \otimes \pi_{B}^{*} \pi_{i}^{*} L_{0}=\pi_{P}^{*} \mathbb{L} \otimes \pi_{B}^{*} F_{\left(\aleph_{B}(\sigma), J_{B}(\sigma)\right)}
$$

Thus, the domain of the immersion $\iota_{\sigma, \sigma}$ induced by $\iota_{\sigma-1, \sigma}$ is

$$
\begin{aligned}
\mathbb{P} \mathcal{N}_{l \sigma-1, \sigma}^{\text {ide }} & =\widetilde{\mathcal{M}}_{1,\left(I_{P}(\sigma), J_{P}(\sigma)\right)} \times \widetilde{\mathfrak{M}}_{0,\left(\aleph_{B}(\sigma), J_{B}(\sigma)\right)}^{0} \\
& =\widetilde{\mathcal{M}}_{1,\left(I_{P}(\sigma), J_{P}(\sigma)\right)} \times \widetilde{\mathfrak{M}}_{0,\left(\aleph_{B}(\sigma), J_{B}(\sigma)\right)}^{\varrho} .
\end{aligned}
$$

By the first statement of Lemma 4.3, an idealized normal bundle for the embedding $\iota_{\sigma, \sigma}$ is the tautological line bundle over $\mathbb{P} \mathcal{N}_{\iota_{\sigma-1, \sigma}}^{\text {ide }}$, ie

$$
\mathcal{N}_{l_{\sigma, \sigma}}^{\text {ide }}=\pi_{P}^{*} \mathbb{L} \otimes \pi_{B}^{*} \gamma_{\left(\aleph_{B}(\sigma), J_{B}(\sigma)\right)}=\pi_{P}^{*} \mathbb{L} \otimes \pi_{B}^{*} \pi_{\varrho_{\sigma}(\sigma)}^{*} \gamma_{\left(\aleph_{B}(\sigma), J_{B}(\sigma)\right)} .
$$

Thus, the inductive assumptions (I4) and (I6), with $\sigma^{\prime}=\sigma$ and $\sigma-1$ replaced by $\sigma$, are satisfied. The same is the case with (I8), since the map $\bar{\iota}_{\sigma-1, \sigma}$ is an embedding by $(I 13)$.

We also note that by the first statement of Lemma 4.9, the inductive assumptions (I1) and (I2), and the last statement of Lemma 3.3,

$$
\iota_{\sigma-1, \sigma}^{-1}\left(\overline{\mathfrak{M}}_{1, \sigma^{*}}^{\sigma-1}\right)=\widetilde{\mathcal{M}}_{1,\left(I_{P}(\sigma), J_{P}(\sigma)\right)} \times\left(\bigcup_{\varrho \in \mathcal{A}_{B}\left(\sigma ; \sigma^{*}\right)} \overline{\mathfrak{M}}_{0, \varrho}\right)
$$

for all $\sigma^{*} \in \mathcal{A}_{1}(d, k)$ such that $\sigma \prec \sigma^{*}$. In addition, by the last statement of Lemma 4.9

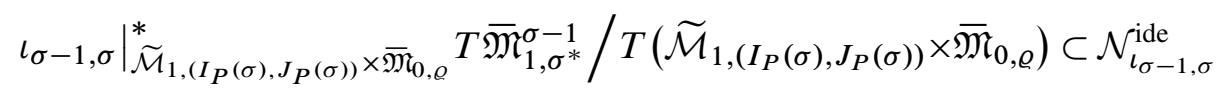

is a vector bundle for all $\varrho \in \mathcal{A}_{B}\left(\sigma ; \sigma^{*}\right)$ and

$$
\begin{aligned}
\left.\iota_{\sigma-1, \sigma}\right|_{\mathcal{M}_{1, I_{P}(\sigma) \sqcup J_{P}(\sigma)}^{*} \times \mathfrak{M}_{0, \varrho}} ^{*} T \overline{\mathfrak{M}}_{1, \sigma^{*}}^{\sigma-1} / T\left(\mathcal{M}_{1,\left(I_{P}(\sigma), J_{P}(\sigma)\right)} \times \mathfrak{M}_{0, \varrho}\right) & \\
& =\bigoplus_{i \in \aleph_{P}(\varrho)} \pi_{P}^{*} L_{i} \otimes \pi_{B}^{*} \pi_{i}^{*} L_{0} .
\end{aligned}
$$


Thus, by the first equality in (4-8),

$$
\begin{aligned}
\left.\iota_{\sigma-1, \sigma}\right|_{\overline{\mathcal{M}}_{1,\left(I_{P}(\sigma), J_{P}(\sigma)\right)}^{*} \times \overline{\mathfrak{M}}_{0, \varrho} T \overline{\mathfrak{M}}_{1, \sigma^{*}}^{\sigma-1} /} T\left(\widetilde{\mathcal{M}}_{1,\left(I_{P}(\sigma), J_{P}(\sigma)\right)} \times \overline{\mathfrak{M}}_{0, \varrho}\right) \\
=\pi_{P}^{*} \mathbb{L} \otimes \bigoplus_{i \in \mathcal{N}_{P}(\varrho)} \pi_{B}^{*} \pi_{i}^{*} L_{0} \\
=\pi_{P}^{*} \mathbb{L} \otimes \pi_{B}^{*} F_{\varrho ; P} .
\end{aligned}
$$

It follows that

$$
\begin{aligned}
& \iota_{\sigma, \sigma}^{-1}\left(\overline{\mathfrak{M}}_{1, \sigma^{*}}^{\sigma}\right)=\left.\bigcup_{\varrho \in \mathcal{A}_{B}\left(\sigma ; \sigma^{*}\right)} \mathbb{P}\left(\pi_{P}^{*} \mathbb{L} \otimes \pi_{B}^{*} F_{\varrho ; P}\right)\right|_{\widetilde{\mathcal{M}}_{1,\left(I_{P}(\sigma), J_{P}(\sigma)\right)} \times \overline{\mathfrak{M}}_{0, \varrho}} \\
& =\widetilde{\mathcal{M}}_{1,\left(I_{P}(\sigma), J_{P}(\sigma)\right)} \times\left(\bigcup_{\varrho \in \mathcal{A}_{B}\left(\sigma ; \sigma^{*}\right)} \mathbb{P} F_{\varrho ; P}\right) \\
& \equiv \widetilde{\mathcal{M}}_{1,\left(I_{P}(\sigma), J_{P}(\sigma)\right)} \times\left(\underset{\varrho \in \mathcal{A}_{B}\left(\sigma ; \sigma^{*}\right)}{\bigcup} \widetilde{\mathfrak{M}}_{0, \varrho}^{0}\right)
\end{aligned}
$$

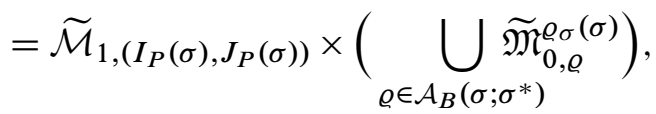

as needed for the inductive assumption (I5) with $\sigma-1$ replaced by $\sigma$ and $\sigma^{\prime}=\sigma$.

Furthermore, by $(I 12), \mathcal{N} \overline{\mathcal{Z}}_{\sigma}^{\sigma-1}$ is the closure of

$$
\begin{aligned}
\left.\mathcal{N} \overline{\mathcal{Z}}_{\sigma}^{0}\right|_{\mathcal{Z}_{\sigma}^{0}} & \left.\equiv \operatorname{ker} \mathcal{D}_{0, \sigma}\right|_{\mathcal{M}_{1, I_{P}(\sigma) \sqcup J_{P}(\sigma)} \times \mathfrak{M}_{0,\left(\aleph_{B}(\sigma), J_{B}(\sigma)\right)}} \\
& =\left.\pi_{P}^{*} \mathbb{L} \otimes \pi_{B}^{*} \operatorname{ker} \mathcal{D}_{\left(\aleph_{B}(\sigma), J_{B}(\sigma)\right)}\right|_{\left.\mathfrak{M}_{0,\left(\aleph_{B}(\sigma), J_{B}\right.}(\sigma)\right)}
\end{aligned}
$$

in $\pi_{P}^{*} \mathbb{L} \otimes \pi_{B}^{*} F_{\left(\aleph_{B}(\sigma), J_{B}(\sigma)\right)}$, where $\mathcal{D}_{\left(\aleph_{B}(\sigma), J_{B}(\sigma)\right)}$ is the bundle homomorphism described in Section 3.3. Thus, by the first statement of Lemma 4.5,

$$
\overline{\mathcal{Z}}_{\sigma}^{\sigma} \equiv \iota_{\sigma, \sigma}^{-1}\left(\overline{\mathfrak{M}}_{1,(0)}^{\sigma}\right)
$$

is the closure of

$$
\mathcal{M}_{1,\left(I_{P}(\sigma), J_{P}(\sigma)\right)} \times\left\{\left.b \in \mathbb{P} F_{\left(\aleph_{B}(\sigma), J_{B}(\sigma)\right)}\right|_{\left.\mathfrak{M}_{0,\left(\aleph_{B}(\sigma), J_{B}(\sigma)\right)}: \widetilde{\mathcal{D}}_{0} b=0\right\}}\right.
$$

in $\widetilde{\mathcal{M}}_{1,\left(I_{P}(\sigma), J_{P}(\sigma)\right)} \times \widetilde{\mathfrak{M}}_{0,\left(\aleph_{B}(\sigma), J_{B}(\sigma)\right)}^{\varrho_{\sigma}(\sigma)}$. The inductive assumption $(I 7)$, with $\sigma^{\prime}=\sigma$ and $\sigma-1$ replaced by $\sigma$, now follows from the first statement of Lemma 3.11.

We next verify that the inductive assumptions (I4)-(I7) hold for $\sigma^{\prime}<\sigma$, with $\sigma-1$ replaced by $\sigma$. If $\sigma^{\prime} \nprec \sigma$, then

$$
\varrho_{\sigma^{\prime}}(\sigma)=\varrho_{\sigma^{\prime}}(\sigma-1) \quad \text { and } \quad \overline{\mathfrak{M}}_{1, \sigma^{\prime}}^{\sigma-1} \cap \overline{\mathfrak{M}}_{1, \sigma}^{\sigma-1}=\varnothing
$$


by definition and (I3), respectively. It then follows that

$$
\begin{gathered}
\iota_{\sigma, \sigma^{\prime}}=\iota_{\sigma-1, \sigma^{\prime}}, \quad \mathcal{N}_{\iota_{\sigma, \sigma^{\prime}}}^{\text {ide }}=\mathcal{N}_{\iota_{\sigma-1, \sigma^{\prime}}}^{\text {ide }}, \\
\overline{\mathfrak{M}}_{1, \sigma^{\prime}}^{\sigma} \cap \overline{\mathfrak{M}}_{1, \sigma^{*}}^{\sigma}=\overline{\mathfrak{M}}_{1, \sigma^{\prime}}^{\sigma-1} \cap \overline{\mathfrak{M}}_{1, \sigma^{*}}^{\sigma-1} \quad \forall \sigma^{*} \in\{(0)\} \sqcup \mathcal{A}_{1}(d, k) .
\end{gathered}
$$

Thus, the inductive assumptions (I4)-(I7), as stated above, imply the corresponding statements with $\sigma-1$ replaced by $\sigma$.

Suppose that $\sigma^{\prime} \prec \sigma$. By (I4) and (1) of Lemma 3.3, the domain of the immersion $\iota_{\sigma, \sigma^{\prime}}$ induced by $\iota_{\sigma-1, \sigma^{\prime}}$ is the blowup of

$$
\widetilde{\mathcal{M}}_{1,\left(I_{P}\left(\sigma^{\prime}\right), J_{P}\left(\sigma^{\prime}\right)\right)} \times \widetilde{\mathfrak{M}}_{0,\left(\aleph_{B}\left(\sigma^{\prime}\right), J_{B}\left(\sigma^{\prime}\right)\right)}^{\varrho}
$$

along the preimage of $\overline{\mathfrak{M}}_{1, \sigma}^{\sigma-1}$ under $\iota_{\sigma-1, \sigma^{\prime}}$ in

$$
\begin{aligned}
& \operatorname{id} \times \pi_{\varrho_{\sigma^{\prime}}(\sigma-1)}: \widetilde{\mathcal{M}}_{1,\left(I_{P}\left(\sigma^{\prime}\right), J_{P}\left(\sigma^{\prime}\right)\right)} \times \widetilde{\mathfrak{M}}_{0,\left(\aleph_{B}\left(\sigma^{\prime}\right), J_{B}\left(\sigma^{\prime}\right)\right)}^{\varrho_{\sigma^{\prime}}(\sigma-1)} \\
& \longrightarrow \widetilde{\mathcal{M}}_{1,\left(I_{P}\left(\sigma^{\prime}\right), J_{P}\left(\sigma^{\prime}\right)\right)} \times \widetilde{\mathfrak{M}}_{0,\left(\aleph_{B}\left(\sigma^{\prime}\right), J_{B}\left(\sigma^{\prime}\right)\right)}^{0}
\end{aligned}
$$

By (I5), this preimage is

$$
\widetilde{\mathcal{M}}_{1,\left(I_{P}\left(\sigma^{\prime}\right), J_{P}\left(\sigma^{\prime}\right)\right)} \times\left(\underset{\varrho \in \mathcal{A}_{B}\left(\sigma^{\prime} ; \sigma\right)}{\bigcup} \widetilde{\mathfrak{M}}_{0, \varrho}^{\varrho_{\sigma^{\prime}}(\sigma-1)}\right) .
$$

By the inductive assumption (I5) in Section 3.4 and the second paragraph after Lemma 4.9,

$$
\widetilde{\mathfrak{M}}_{0, \varrho_{1}}^{\varrho_{\sigma^{\prime}}(\sigma-1)} \cap \widetilde{\mathfrak{M}}_{0, \varrho_{2}}^{\varrho_{\sigma^{\prime}}(\sigma-1)}=\varnothing \quad \forall \varrho_{1}, \varrho_{2} \in \mathcal{A}_{B}\left(\sigma^{\prime} ; \sigma\right), \varrho_{1} \neq \varrho_{2} .
$$

Thus, by the construction of Section 3.4, the blowup of $\widetilde{\mathfrak{M}}_{0,\left(\boldsymbol{\aleph}_{B}\left(\sigma^{\prime}\right), J_{B}\left(\sigma^{\prime}\right)\right)}^{\varrho_{\sigma^{\prime}}(\sigma-1)}$ along

$$
\bigcup_{\varrho \in \mathcal{A}_{B}\left(\sigma^{\prime} ; \sigma\right)} \widetilde{\mathfrak{M}}_{0, \varrho}^{\varrho_{\sigma^{\prime}}(\sigma-1)}
$$

is $\widetilde{\mathfrak{M}}_{0,\left(\aleph_{B}\left(\sigma^{\prime}\right), J_{B}\left(\sigma^{\prime}\right)\right)}^{\varrho_{\sigma^{\prime}}(\sigma)}$, as needed for the inductive statement (I4), with $\sigma-1$ replaced by $\sigma$. The inductive requirement (I5) is obtained by the same reasoning, using the last statement of Lemma 3.3.

Since $\overline{\mathfrak{M}}_{1, \sigma}^{\sigma-1}$ is not contained in $\overline{\mathfrak{M}}_{1, \sigma^{\prime}}^{\sigma-1}$, the bundle homomorphism

$$
\iota_{\sigma-1, \sigma^{\prime}}^{*} T \overline{\mathfrak{M}}_{1, \sigma}^{\sigma-1} \longrightarrow \mathcal{N}_{\iota_{\sigma-1, \sigma^{\prime}}}^{\text {ide }}
$$

must be surjective on every fiber over $\iota_{\sigma-1, \sigma^{\prime}}^{-1}\left(\overline{\mathfrak{M}}_{1, \sigma}^{\sigma-1}\right)$ by (I14). Thus, the inductive assumption (I6), for $\sigma^{\prime}<\sigma$, continues to hold. Furthermore, by (I7) and the last 
statement of Lemma 3.3, $\overline{\mathcal{Z}}_{\sigma^{\prime}}^{\sigma}$ is the closure of

$$
\begin{aligned}
& \widetilde{\mathcal{M}}_{1,\left(I_{P}\left(\sigma^{\prime}\right), J_{P}\left(\sigma^{\prime}\right)\right)} \times\left(\widetilde{\mathcal{D}}_{\varrho_{\sigma^{\prime}}(\sigma-1)}^{-1}(0)-\bigcup_{\substack{\alpha \in \mathcal{A}_{B}\left(\aleph_{B}\left(\sigma^{\prime}\right) ; d, J_{B}\left(\sigma^{\prime}\right)\right) \\
\varrho_{\sigma^{\prime}}(\sigma-1)<\varrho}} \widetilde{\mathfrak{M}}_{0, \varrho}^{\varrho_{\sigma^{\prime}}(\sigma-1)}\right)
\end{aligned}
$$

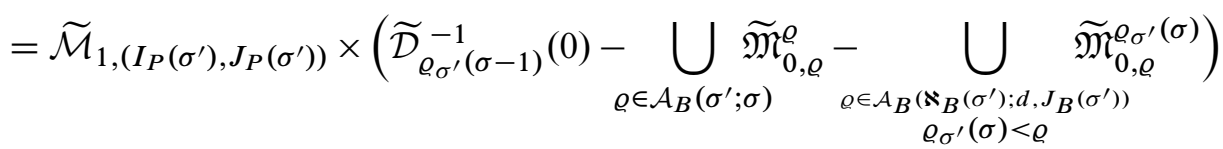

in $\widetilde{\mathcal{M}}_{1,\left(I_{P}\left(\sigma^{\prime}\right), J_{P}\left(\sigma^{\prime}\right)\right)} \times \widetilde{\mathfrak{M}}_{0,\left(\aleph_{B}\left(\sigma^{\prime}\right), J_{B}\left(\sigma^{\prime}\right)\right)}^{\varrho_{\sigma^{\prime}}(\sigma)}$. By the construction of Section 3.4,

$$
\begin{aligned}
& \left.\widetilde{\mathcal{D}}_{\varrho_{\sigma^{\prime}}(\sigma-1)}\right|_{\widetilde{\mathfrak{M}}_{0,\left(\aleph_{B}\left(\sigma^{\prime}\right), J_{B}\left(\sigma^{\prime}\right)\right)}}-\bigcup_{\varrho \in \mathcal{A}_{B}\left(\sigma^{\prime} ; \sigma\right)} \widetilde{\mathfrak{M}}_{0, \varrho}^{\varrho} \\
& =\left.\widetilde{\mathcal{D}}_{\varrho_{\sigma^{\prime}}(\sigma)}\right|_{\widetilde{\mathfrak{M}}_{0,\left(\aleph_{B} \sigma^{\prime}\left(\sigma^{\prime}\right), J_{B}\left(\sigma^{\prime}\right)\right)}^{(\sigma)}}-\bigcup_{\varrho \in \mathcal{A}_{B}\left(\sigma^{\prime} ; \sigma\right)} \widetilde{\mathfrak{M}}_{0, \varrho}^{\varrho} \cdot
\end{aligned}
$$

Since $\widetilde{\mathcal{D}}_{\varrho_{\sigma^{\prime}}(\sigma)}$ is transverse to the zero set outside of the subvarieties $\widetilde{\mathfrak{M}}_{0, \varrho}^{\varrho_{\sigma^{\prime}}(\sigma)}$ with $\varrho>\varrho_{\sigma^{\prime}}(\sigma)$ by the inductive requirement (I4) in Section 3.4, we conclude that the first part of the inductive assumption (I7), with $\sigma-1$ replaced by $\sigma$, is satisfied. The second part follows from the last statement of Lemma 4.5.

It remains to verify the inductive assumption (I9)-(I12), with $\sigma-1$ replaced by $\sigma$. Suppose $\sigma^{*} \in \mathcal{A}_{1}(d, k)$ is such that $\sigma<\sigma^{*}$. If $\sigma \nprec \sigma^{*}$, then

$$
\rho_{\sigma^{*}}(\sigma)=\rho_{\sigma^{*}}(\sigma-1) \quad \text { and } \quad \overline{\mathfrak{M}}_{1, \sigma^{*}}^{\sigma-1} \cap \overline{\mathfrak{M}}_{1, \sigma}^{\sigma-1}=\varnothing,
$$

by definition and (I3), respectively. It then follows that

$$
\begin{gathered}
\iota_{\sigma, \sigma^{*}}=\iota_{\sigma-1, \sigma^{*}}, \quad \mathcal{N}_{\iota_{\sigma, \sigma^{*}}}^{\text {ide }}=\mathcal{N}_{\iota_{\sigma-1, \sigma^{*}}}^{\text {ide }} \\
\overline{\mathfrak{M}}_{1, \sigma^{*}}^{\sigma} \cap \overline{\mathfrak{M}}_{1, \sigma^{\prime}}^{\sigma}=\overline{\mathfrak{M}}_{1, \sigma^{*}}^{\sigma-1} \cap \overline{\mathfrak{M}}_{1, \sigma^{\prime}}^{\sigma-1} \quad \forall \sigma^{\prime} \in\{(0)\} \sqcup \mathcal{A}_{1}(d, k) .
\end{gathered}
$$

Thus, the inductive assumptions (I9)-(I12), as stated above, imply the corresponding statements with $\sigma-1$ replaced by $\sigma$.

Suppose that $\sigma \prec \sigma^{*}$. By (I9) and (1) of Lemma 3.3, the domain of the immersion $\iota_{\sigma, \sigma^{*}}$ induced by $\iota_{\sigma-1, \sigma^{*}}$ is the blowup of

$$
\overline{\mathcal{M}}_{1,\left(I_{P}\left(\sigma^{*}\right), J_{P}\left(\sigma^{*}\right)\right)}^{\rho_{\sigma^{*}}(\sigma-1)} \times \overline{\mathfrak{M}}_{0,\left(\aleph_{B}\left(\sigma^{*}\right), J_{B}\left(\sigma^{*}\right)\right)}
$$

along the preimage of $\overline{\mathfrak{M}}_{1, \sigma}^{\sigma-1}$ under $\iota_{\sigma-1, \sigma^{*}}$ in

$$
\begin{aligned}
\pi_{\rho_{\sigma^{*}}(\sigma-1)} \times \mathrm{id}: \overline{\mathcal{M}}_{1,\left(I_{P}\left(\sigma^{*}\right), J_{P}\left(\sigma^{*}\right)\right)}^{\rho_{\sigma^{*}}(\sigma-1)} & \times \overline{\mathfrak{M}}_{0,\left(\aleph_{B}\left(\sigma^{*}\right), J_{B}\left(\sigma^{*}\right)\right)} \\
& \longrightarrow \overline{\mathcal{M}}_{1, I_{P}\left(\sigma^{*}\right) \sqcup J_{P}\left(\sigma^{*}\right)} \times \overline{\mathfrak{M}}_{0,\left(\aleph_{B}\left(\sigma^{*}\right), J_{B}\left(\sigma^{*}\right)\right)}
\end{aligned}
$$


By ( $I 10)$, this preimage is

$$
\left(\bigcup_{\rho \in \mathcal{A}_{P}\left(\sigma^{*} ; \sigma\right)} \overline{\mathcal{M}}_{1, \rho}^{\rho^{*}(\sigma-1)}\right) \times \overline{\mathfrak{M}}_{0,\left(\aleph_{B}\left(\sigma^{*}\right), J_{B}\left(\sigma^{*}\right)\right)} .
$$

By Lemma 2.6 and the paragraph after Lemma 4.9,

$$
\overline{\mathcal{M}}_{1, \rho_{1}}^{\rho_{\sigma^{*}}(\sigma-1)} \cap \overline{\mathcal{M}}_{1, \rho_{2}}^{\rho_{\sigma^{*}}(\sigma-1)}=\varnothing \quad \forall \rho_{1}, \rho_{2} \in \mathcal{A}_{P}\left(\sigma^{*} ; \sigma\right), \rho_{1} \neq \rho_{2} .
$$

Thus, by the construction of Section 2.3, the blowup of $\overline{\mathcal{M}}_{1,\left(I_{P}\left(\sigma^{*}\right), J_{P}\left(\sigma^{*}\right)\right)}^{\rho_{\sigma^{*}}(\sigma-1)}$ along

$$
\bigcup_{\rho \in \mathcal{A}_{P}\left(\sigma^{*} ; \sigma\right)} \overline{\mathcal{M}}_{1, \rho}^{\rho_{\sigma^{*}}(\sigma-1)}
$$

is $\overline{\mathcal{M}}_{1,\left(I_{P}\left(\sigma^{*}\right), J_{P}\left(\sigma^{*}\right)\right)}^{\rho_{\sigma *}(\sigma)}$, as needed for the inductive statement (I9), with $\sigma-1$ replaced by $\sigma$. The inductive assumptions (I10) and (I11) are verified similarly, using the last statement of Lemma 3.3 and Lemma 4.3. The argument for $(I 11)$ is nearly identical to the verification of the inductive assumption $(I 11)$ in Section 3.4. Finally, the inductive requirement (I12), with $\sigma-1$ replaced by $\sigma$, follows from the last statement of Lemma 4.5, along with the assumptions (I1) and (I2).

We conclude this blowup construction after the $\sigma_{\max }$ step and put

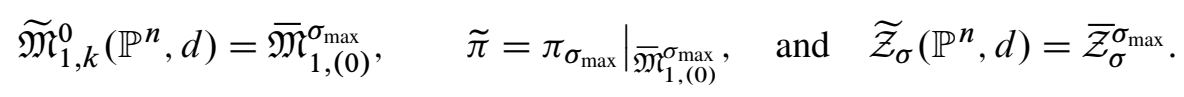

The inductive assumptions ( $I 1)-(I 8)$ imply that

$$
\tilde{\pi}: \widetilde{\mathfrak{M}}_{1, k}^{0}\left(\mathbb{P}^{n}, d\right) \longrightarrow \overline{\mathfrak{M}}_{1, k}^{0}\left(\mathbb{P}^{n}, d\right)
$$

is a desingularization as described in Section 1.2. By (I3), the final result of this blowup construction is independent of the choice of full ordering $<$ extending the natural partial ordering $\prec$ on $\mathcal{A}_{1}(d, k)$.

\section{Proof of Theorem 1.2}

\subsection{Pushforwards of vector bundles}

In this section we prove Theorem 1.2 by lifting the construction of Section 4 from stable maps into $\mathbb{P}^{n}$ to stable maps into (the total space of) the line bundle $\mathcal{L}$ associated to the locally free sheaf $\mathcal{O}_{\mathbb{P}^{n}}(a)$.

Let $\tau: \mathcal{L} \longrightarrow \mathbb{P}^{n}$ be the bundle projection map. We denote by $\overline{\mathfrak{M}}_{1, k}(\mathcal{L}, d)$ the moduli space of degree- $d$ stable maps from genus-one curves with $k$ marked points into $\mathcal{L}$. 
The projection map $\tau$ induces a morphism,

$$
p: \overline{\mathfrak{M}}_{1, k}(\mathcal{L}, d) \longrightarrow \overline{\mathfrak{M}}_{1, k}\left(\mathbb{P}^{n}, d\right), \quad[\Sigma, u] \longrightarrow[\Sigma, \tau \circ u] .
$$

Since no fiber of $\mathcal{L}$ contains the image of a nonconstant holomorphic map, the ghost components of $(\Sigma, \tau \circ u)$ are precisely the same as the ghost components of $(\Sigma, u)$. We note that

$$
p^{-1}([\Sigma, u])=H^{0}\left(\Sigma ; u^{*} \mathcal{L}\right) / \operatorname{Aut}(\Sigma, u) .
$$

In particular, $p$ is a bundle of vector spaces, but of two possible ranks: $d a$ and $d a+1$. Let $\mathcal{S}_{\mathcal{L}}$ denote the sheaf of (holomorphic) sections of

$$
p\left|\overline{\mathfrak{M}}_{1, k}^{0}\left(\mathbb{P}^{n}, d\right): \overline{\mathfrak{M}}_{1, k}(\mathcal{L}, d)\right|_{\overline{\mathfrak{M}}_{1, k}^{0}\left(\mathbb{P}^{n}, d\right)} \longrightarrow \overline{\mathfrak{M}}_{1, k}^{0}\left(\mathbb{P}^{n}, d\right) .
$$

Similarly, denote by $\widetilde{S}_{\mathcal{L}}$ the sheaf of sections of

$$
p: \tilde{\pi}^{*} \overline{\mathfrak{M}}_{1, k}(\mathcal{L}, d) \longrightarrow \widetilde{\mathfrak{M}}_{1, k}^{0}\left(\mathbb{P}^{n}, d\right),
$$

where $\tilde{\pi}: \widetilde{\mathfrak{M}}_{1, k}^{0}\left(\mathbb{P}^{n}, d\right) \longrightarrow \overline{\mathfrak{M}}_{1, k}^{0}\left(\mathbb{P}^{n}, d\right)$ is the desingularization map of Theorem 1.1:

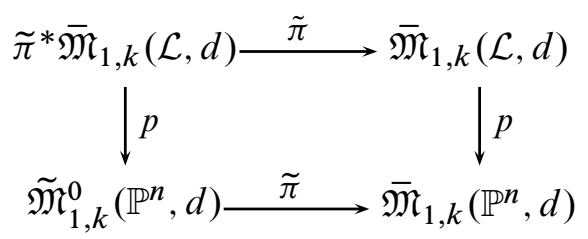

Lemma 5.1 With notation as in Theorem 1.2 and above,

(1) the sheaves $\mathcal{S}_{\mathcal{L}}$ and $\pi_{*} \mathrm{ev}^{*} \mathcal{O}_{\mathbb{P}^{n}}(a)$ over $\overline{\mathfrak{M}}_{1, k}^{0}\left(\mathbb{P}^{n}, d\right)$ are isomorphic;

(2) the sheaves $\widetilde{\mathcal{S}}_{\mathcal{L}}$ and $\pi_{*} \tilde{\pi}^{*} \operatorname{ev}^{*} \mathcal{O}_{\mathbb{P}^{n}}(a)$ over $\widetilde{\mathfrak{M}}_{1, k}^{0}\left(\mathbb{P}^{n}, d\right)$ are isomorphic.

Let $\mathfrak{U}_{\mathcal{L}}$ be the universal curve over $\overline{\mathfrak{M}}_{1, k}(\mathcal{L}, d) \mid \overline{\mathfrak{M}}_{1, k}^{0}\left(\mathbb{P}^{n}, d\right)$, with structure map $\pi_{\mathcal{L}}$ and evaluation map ev $\operatorname{ve}_{\mathcal{L}}$. The projection map $\tau$ induces a morphism $\widetilde{p}$ on $\mathfrak{U}_{\mathcal{L}}$ so that the diagram

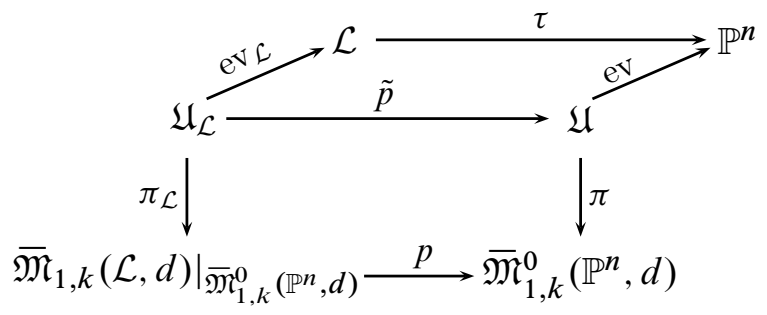


commutes. Suppose $W \subset \overline{\mathfrak{M}}_{1, k}^{0}\left(\mathbb{P}^{n}, d\right)$ is an open subset.

(i) An element

$$
s \in\left\{\pi_{*} \mathrm{ev}^{*} \mathcal{O}_{\mathbb{P}^{n}}(a)\right\}(W) \equiv H^{0}\left(\pi^{-1}(W) ; \mathrm{ev}^{*} \mathcal{L}\right)
$$

induces a morphism $\tilde{s}: \pi^{-1}(W) \longrightarrow \mathcal{L}$ so that ev $=\tau \circ \tilde{s}$. In turn, $\tilde{s}$ induces morphisms $f_{s}$ and $\widetilde{f}_{s}$ to $\overline{\mathfrak{M}}_{1, k}(\mathcal{L}, d) \mid \overline{\mathfrak{M}}_{1, k}^{0}\left(\mathbb{P}^{n}, d\right)$ and $\mathfrak{U}_{\mathcal{L}}$

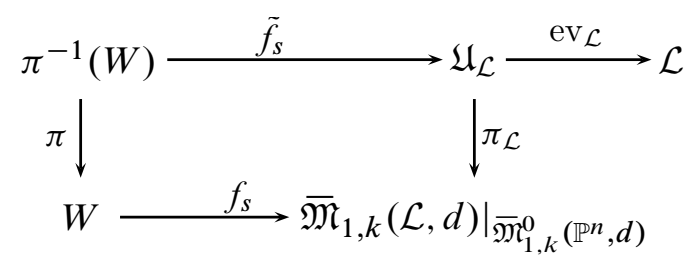

so that $\tilde{s}=\operatorname{ev} \mathcal{L}^{\circ} \tilde{f}_{s}$. Then

$\mathrm{ev} \circ \tilde{p} \circ \tilde{f}_{s}=\tau \circ \mathrm{ev}_{\mathcal{L}} \circ \tilde{f}_{s}=\tau \circ \tilde{s}=\mathrm{ev}: \pi^{-1}(W) \longrightarrow \mathbb{P}^{n} \quad \Longrightarrow \quad p \circ f_{s}=\operatorname{id}_{W}$, since $\pi \circ \tilde{p} \circ \tilde{f}_{s}=p \circ f_{s} \circ \pi$. Thus, $f_{s} \in \mathcal{S}_{\mathcal{L}}(W)$. It is immediate that the map

$$
\left\{\pi_{*} \mathrm{ev}^{*} \mathcal{O}_{\mathbb{P}^{n}}(a)\right\}(W) \longrightarrow \mathcal{S}_{\mathcal{L}}(W), \quad s \longrightarrow f_{s},
$$

induces a sheaf homomorphism.

(ii) Conversely, let $\sigma \in \mathcal{S}_{\mathcal{L}}(W)$, ie $\sigma: W \longrightarrow \overline{\mathfrak{M}}_{1, k}(\mathcal{L}, d)$ is a morphism such that $p \circ \sigma=\operatorname{id}_{W}$. Since $\mathfrak{U}_{\mathcal{L}}=p^{*} \mathfrak{U}$,

$$
\left.\pi^{-1}(W) \equiv \mathfrak{U}\right|_{W}=\sigma^{*} \mathfrak{U}_{\mathcal{L}}
$$

Thus, $\sigma$ lifts to a morphism

$$
\tilde{\sigma}: \pi^{-1}(W)=\sigma^{*} \mathfrak{U}_{\mathcal{L}} \longrightarrow \mathfrak{U}_{\mathcal{L}} .
$$

Let $g_{\sigma}=\operatorname{ev}_{\mathcal{L}} \circ \tilde{\sigma}$. Then

$$
\tau \circ g_{\sigma}=\tau \circ \mathrm{ev}_{\mathcal{L}} \circ \tilde{\sigma}=\mathrm{ev} \circ \tilde{p} \circ \tilde{\sigma}=\mathrm{ev},
$$

ie $g_{\sigma} \in H^{0}\left(\pi^{-1}(W)\right.$; ev* $\left.\mathcal{L}\right)$. It is immediate that the map

$$
\mathcal{S}_{\mathcal{L}}(W) \longrightarrow\left\{\pi_{*} \mathrm{ev}^{*} \mathcal{O}_{\mathbb{P}^{n}}(a)\right\}(W), \quad \sigma \longrightarrow g_{\sigma},
$$

induces a sheaf homomorphism. Furthermore,

$$
g_{f_{s}}=s \quad \forall s \in\left\{\pi_{*} \mathrm{ev}^{*} \mathcal{O}_{\mathbb{P}^{n}}(a)\right\}(W) \quad \text { and } \quad f_{g_{\sigma}}=\sigma \quad \forall \sigma \in \mathcal{S}_{\mathcal{L}}(W) .
$$


These observations imply the first statement of Lemma 5.1. The second claim is proved similarly.

Let

$$
\overline{\mathfrak{M}}_{1, k}^{0}(\mathcal{L}, d) \subset \overline{\mathfrak{M}}_{1, k}(\mathcal{L}, d)
$$

be the closure of the locus of maps from smooth domains. We show in Section 5.4 that the proper transform $\widetilde{\mathfrak{M}}_{1, k}^{0}(\mathcal{L}, d)$ of $\overline{\mathfrak{M}}_{1, k}^{0}(\mathcal{L}, d)$ in

$$
\tilde{\pi}^{*} \overline{\mathfrak{M}}_{1, k}(\mathcal{L}, d) \longrightarrow \widetilde{\mathfrak{M}}_{1, k}^{0}\left(\mathbb{P}^{n}, d\right)
$$

is smooth. Similarly to the case of $\widetilde{\mathfrak{M}}_{1, k}^{0}\left(\mathbb{P}^{n}, d\right)$, the main stratum of $\widetilde{\mathfrak{M}}_{1, k}^{0}(\mathcal{L}, d)$,

$$
\left.\mathfrak{M}_{1, k}^{\mathrm{eff}}(\mathcal{L}, d) \equiv \overline{\mathfrak{M}}_{1, k}(\mathcal{L}, d)\right|_{\mathfrak{M}_{1, k}^{\mathrm{eff}}\left(\mathbb{P}^{n}, d\right)}=\widetilde{\mathfrak{M}}_{1, k}^{0}(\mathcal{L}, d)-\bigcup_{\sigma \in \mathcal{A}_{1}(d, k)} p^{-1}\left(\operatorname{Im} \iota_{\sigma_{\max }, \sigma}\right)
$$

is smooth. On the other hand, by the inductive assumption $(I 1)$ and the last paragraph of Section 5.4, for each $\sigma \in \mathcal{A}_{1}(d, k)$

$$
\widetilde{\mathfrak{M}}_{1, k}^{0}(\mathcal{L}, d) \cap p^{-1}\left(\operatorname{Im} \iota_{\sigma_{\max }, \sigma}\right)
$$

is the image of a smooth variety under the bundle homomorphism $\bar{j}_{\sigma_{\max }, \sigma}$ lifting the embedding $\bar{\iota}_{\sigma_{\max }, \sigma}$ of Section 4.3. Thus,

$$
\widetilde{\mathfrak{M}}_{1, k}^{0}(\mathcal{L}, d) \cap p^{-1}\left(\operatorname{Im} \iota_{\sigma_{\max }, \sigma}\right)
$$

is a smooth subvariety of $\widetilde{\mathfrak{M}}_{1, k}^{0}(\mathcal{L}, d)$. As its normal cone in $\widetilde{\mathfrak{M}}_{1, k}^{0}(\mathcal{L}, d)$ is a line bundle by the inductive assumption $(I 1)$ of Section 5.4 for every $\sigma \in \mathcal{A}_{1}(d, k)$, we conclude that the entire space $\widetilde{\mathfrak{M}}_{1, k}^{0}(\mathcal{L}, d)$ is smooth. Furthermore, the fibers of

$$
\widetilde{p}: \widetilde{\mathfrak{M}}_{1, k}^{0}(\mathcal{L}, d) \longrightarrow \widetilde{\mathfrak{M}}_{1, k}^{0}\left(\mathbb{P}^{n}, d\right)
$$

are vector spaces of the same rank and $\widetilde{\mathfrak{M}}_{1, k}^{0}(\mathcal{L}, d)$ contains $\widetilde{\mathfrak{M}}_{1, k}^{0}\left(\mathbb{P}^{n}, d\right)$ as the zero section. Thus, $\tilde{p}$ is a vector bundle.

Lemma 5.1 and the previous paragraph imply (1) of Theorem 1.2. The second claim of this theorem is obtained in the last paragraph of Section 5.4. Finally, (3) of Theorem 1.2 follows from (4) of Theorem 1.1 and the following lemma. 
Lemma 5.2 Suppose $\tilde{\pi}: \widetilde{\mathfrak{M}} \longrightarrow \overline{\mathfrak{M}}$ is a morphism between varieties, $\mathfrak{U} \longrightarrow \overline{\mathfrak{M}}$ is a flat family of curves, $\mathcal{L} \longrightarrow \mathfrak{U}$ is a line bundle, and $\pi: \widetilde{\mathfrak{U}} \longrightarrow \widetilde{\mathfrak{M}}$ and $\widetilde{\mathcal{L}} \longrightarrow \widetilde{\mathfrak{U}}$ are the pullbacks of $\mathfrak{U}$ and $\mathcal{L}$ via $\tilde{\pi}$ :

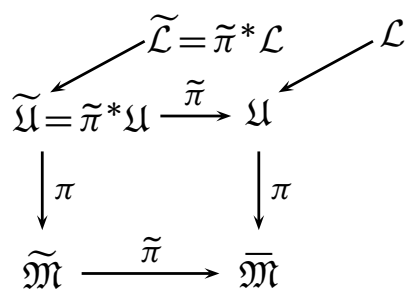

If the morphism $\tilde{\pi}$ is surjective and its fibers are compact and connected, then

$$
\tilde{\pi}_{*} \pi_{*} \widetilde{\mathcal{L}}=\pi_{*} \mathcal{L} .
$$

Since $\mathcal{L}$ is locally trivial, Lemma 5.2 follows from

$$
\tilde{\pi}_{*} \mathcal{O}_{\widetilde{\mathfrak{U}}}=\mathcal{O}_{\mathfrak{U}} \text {. }
$$

In turn, this identity follows from the fact that every holomorphic function on a compact connected variety is constant. Thus, if $\widetilde{W} \subset \mathfrak{U}$ is any open subset and $\widetilde{f}$ is a holomorphic function on $\tilde{\pi}^{-1}(\widetilde{W}) \subset \widetilde{\mathfrak{U}}$, then $\tilde{f}$ is constant on the fibers of $\tilde{\pi}$, ie $\tilde{f}=\tilde{\pi}^{*} f$ for some holomorphic function $f$ on $\widetilde{W}$.

\subsection{Construction of bundle homomorphism}

In this subsection we describe the surjective bundle homomorphism that appears in the second statement of Theorem 1.2; see Proposition 5.5. The construction of this homomorphism is similar to the construction of the homomorphism $\widetilde{\mathcal{D}}_{(\aleph, J)}$ in Sections 3.3 and 3.4 .

Let $\mathcal{L} \longrightarrow \mathbb{P}^{n}$ be a line bundle as in Section 1.2. If $J$ is a finite set, let

$$
\mathcal{V}_{0}=\overline{\mathfrak{M}}_{0,\{0\} \sqcup J}(\mathcal{L}, d) \longrightarrow \overline{\mathfrak{M}}_{0,\{0\} \sqcup J}\left(\mathbb{P}^{n}, d\right)
$$

be the corresponding cone. In particular, if $[\Sigma, u] \in \overline{\mathfrak{M}}_{0,\{0\} \sqcup J}\left(\mathbb{P}^{n}, d\right)$, then

$$
\left.\mathcal{V}_{0}\right|_{[\Sigma, u]}=H^{0}\left(\Sigma ; u^{*} \mathcal{L}\right) / \operatorname{Aut}(\Sigma, u) .
$$

In this, genus-zero, case, this is a vector bundle of the expected rank. Let

$$
\nabla^{u}: \Gamma\left(\Sigma ; u^{*} \mathcal{L}\right) \longrightarrow \Gamma\left(\Sigma ; T^{*} \Sigma \otimes u^{*} \mathcal{L}\right)
$$


be the pullback of the standard Hermitian connection in $\mathcal{L}$ by $u$. We define

$$
\begin{aligned}
\mathfrak{D}_{0} \in & \Gamma\left(\overline{\mathfrak{M}}_{0,\{0\} \sqcup J}\left(\mathbb{P}^{n}, d\right) ; \operatorname{Hom}\left(L_{0} \otimes \mathcal{V}_{0}, \mathrm{ev}_{0}^{*} \mathcal{L}\right)\right) \\
& =\Gamma\left(\overline{\mathfrak{M}}_{0,\{0\} \sqcup J}\left(\mathbb{P}^{n}, d\right) ; \operatorname{Hom}\left(L_{0}, \operatorname{Hom}\left(\mathcal{V}_{0}, \mathrm{ev}_{0}^{*} \mathcal{L}\right)\right)\right) \\
& =\Gamma\left(\overline{\mathfrak{M}}_{0,\{0\} \sqcup J}\left(\mathbb{P}^{n}, d\right) ; \operatorname{Hom}\left(\mathcal{V}_{0}, \operatorname{Hom}\left(L_{0}, \mathrm{ev}_{0}^{*} \mathcal{L}\right)\right)\right) \\
\mathfrak{D}_{0} \xi= & \left.\nabla^{u} \xi\right|_{x_{0}(\Sigma, u)} \quad \forall \xi \in H^{0}\left(\Sigma ; u^{*} \mathcal{L}\right),
\end{aligned}
$$

by

where $x_{0}(\Sigma, u) \in \Sigma$ is the marked point labeled by 0 as before. We note that $\mathfrak{D}_{0}$ vanishes identically on the subvarieties $\overline{\mathfrak{M}}_{0, \sigma}\left(\mathbb{P}^{n}, d\right)$ with $\sigma \in \mathcal{A}_{0}(d, J)$ defined in Section 3.2.

If $\aleph$ and $J$ are finite sets, let

$$
p_{(\aleph, J)}: \mathcal{V}_{(\aleph, J)} \longrightarrow \overline{\mathfrak{M}}_{0,(\aleph, J)}\left(\mathbb{P}^{n}, d\right)
$$

be the vector bundle induced by $\mathcal{L}$, where $\overline{\mathfrak{M}}_{0,(\aleph, J)}\left(\mathbb{P}^{n}, d\right)$ is as in Section 3.3. It is immediate that

$$
\mathcal{V}_{(\boldsymbol{\aleph}, J)}=\left\{\left(\xi_{i}\right)_{i \in \boldsymbol{N}} \in \bigoplus_{i \in \boldsymbol{N}} \pi_{i}^{*} \mathcal{V}_{0}: \operatorname{ev}_{0}\left(\xi_{i}\right)=\operatorname{ev}_{0}\left(\xi_{i^{\prime}}\right) \forall i, i^{\prime} \in \boldsymbol{\aleph}\right\}=\overline{\mathfrak{M}}_{0,(\boldsymbol{\aleph}, J)}(\mathcal{L}, d)
$$

Note that for every $\sigma=\left(m ; J_{P}, J_{B}\right) \in \mathcal{A}_{0}(d, J)$,

$$
\iota_{\sigma}^{*} \mathcal{V}_{0}=\pi_{B}^{*} \mathcal{V}_{\left([m], J_{B}\right)} \longrightarrow \overline{\mathcal{M}}_{0,\{0\} \sqcup[m] \sqcup J_{B}} \times \overline{\mathfrak{M}}_{0,([m], J)}\left(\mathbb{P}^{n}, d\right)
$$

where $\iota_{\sigma}$ is as in Section 3.2.

Lemma 5.3 If $d \in \mathbb{Z}^{+}, J, \mathcal{L}$, and $\mathcal{V}_{0}$ are as above, the bundle homomorphism

$$
\mathfrak{D}_{0} \in \Gamma\left(\overline{\mathfrak{M}}_{0,\{0\} \sqcup J}\left(\mathbb{P}^{n}, d\right) ; \operatorname{Hom}\left(\mathcal{V}_{0}, L_{0}^{*} \otimes \operatorname{ev}_{0}^{*} \mathcal{L}\right)\right)
$$

is surjective on the complement of the subvarieties $\overline{\mathfrak{M}}_{0, \sigma}\left(\mathbb{P}^{n}, d\right)$ with $\sigma \in \mathcal{A}_{0}(d, J)$. Furthermore, for every

$$
\sigma \equiv\left(m ; J_{P}, J_{B}\right) \in \mathcal{A}_{0}(d, J)
$$

the differential of $\mathfrak{D}_{0}$,

$$
\nabla \mathfrak{D}_{0}: \mathcal{N}_{\iota_{\sigma}} \longrightarrow \iota_{\sigma}^{*} \operatorname{Hom}\left(\mathcal{V}_{0}, L_{0}^{*} \otimes \mathrm{ev}_{0}^{*} \mathcal{L}\right)=\pi_{P}^{*} L_{0}^{*} \otimes \pi_{B}^{*} \operatorname{Hom}\left(\mathcal{V}_{\left([m], J_{B}\right)}, \mathrm{ev}_{0}^{*} \mathcal{L}\right),
$$

in the normal direction to the immersion $\iota_{\sigma}$ is given by

$$
\left.\nabla \mathfrak{D}_{0}\right|_{\pi_{P}^{*} L_{i} \otimes \pi_{B}^{*} \pi_{i}^{*} L_{0}}=\pi_{P}^{*} s_{i} \otimes \pi_{B}^{*} \pi_{i}^{*} \mathfrak{D}_{0} \quad \forall i \in[m],
$$

where $s_{i}$ is the homomorphism defined in Section 2.2. 
Lemma 5.3 can viewed as the analogue of Lemma 3.8 for vector bundle sections. The first claim of Lemma 5.3 is an immediate consequence of the fact that

$$
H^{1}\left(\Sigma ; u^{*} \mathcal{L} \otimes \mathcal{O}(-2 z)\right)=\{0\}
$$

for every genus-zero stable map $(\Sigma, u)$ and a smooth point $z \in \Sigma$ such that the restriction of $u$ to the irreducible component of $\Sigma$ containing $z$ is not constant. The second statement follows from [25, Lemma 4.2].

With notation as in Section 3.3, let

$$
\begin{aligned}
\mathfrak{D}_{(\aleph, J)} \in & \Gamma\left(\overline{\mathfrak{M}}_{0,(\aleph, J)}\left(\mathbb{P}^{n}, d\right) ; \operatorname{Hom}\left(\mathcal{V}_{(\aleph, J)}, \operatorname{Hom}\left(F_{(\aleph, J)}, \operatorname{ev}_{0}^{*} \mathcal{L}\right)\right)\right) \\
& =\Gamma\left(\overline{\mathfrak{M}}_{0,(\aleph, J)}\left(\mathbb{P}^{n}, d\right) ; \operatorname{Hom}\left(F_{(\aleph, J)}, \operatorname{Hom}\left(\mathcal{V}_{(\aleph, J)}, \operatorname{ev}_{0}^{*} \mathcal{L}\right)\right)\right) \\
& =\Gamma\left(\overline{\mathfrak{M}}_{0,(\boldsymbol{\aleph}, J)}\left(\mathbb{P}^{n}, d\right) ; \operatorname{Hom}\left(F_{(\aleph, J)} \otimes \mathcal{V}_{(\aleph, J)}, \operatorname{ev}_{0}^{*} \mathcal{L}\right)\right)
\end{aligned}
$$

be the homomorphism defined by

$$
\left.\mathfrak{D}_{(\aleph, J)}\right|_{\pi_{i}^{*} L_{0} \otimes \pi_{j}^{*} \mathcal{V}_{0}}=\left\{\begin{array}{ll}
\pi_{i}^{*} \mathfrak{D}_{0}, & \text { if } j=i ; \\
0, & \text { otherwise; }
\end{array} \quad \forall i, j \in \boldsymbol{\aleph} .\right.
$$

It induces a section

$$
\begin{aligned}
\widetilde{\mathfrak{D}}_{0} \in & \Gamma\left(\widetilde{\mathfrak{M}}_{0,(\aleph, J)}^{0}\left(\mathbb{P}^{n}, d\right) ; \operatorname{Hom}\left(\gamma_{(\aleph, J)}, \pi_{\mathbb{P} F_{(\aleph, J)}}^{*} \operatorname{Hom}\left(\mathcal{V}_{(\aleph, J)}, \mathrm{ev}_{0}^{*} \mathcal{L}\right)\right)\right) \\
& =\Gamma\left(\widetilde{\mathfrak{M}}_{0,(\aleph, J)}^{0}\left(\mathbb{P}^{n}, d\right) ; \operatorname{Hom}\left(\pi_{\mathbb{P} F_{(\aleph, J)}^{*}}^{*} \mathcal{V}_{(\aleph, J)}, \mathbb{E}_{0}^{*} \otimes \pi_{F_{(\aleph, J)}}^{*} \operatorname{ev}_{0}^{*} \mathcal{L}\right)\right)
\end{aligned}
$$

This section vanishes identically on the subvarieties $\widetilde{\mathfrak{M}}_{0, \varrho}^{0}\left(\mathbb{P}^{n}, d\right)$ of $\widetilde{\mathfrak{M}}_{0,(\aleph, J)}^{0}\left(\mathbb{P}^{n}, d\right)$ with $\varrho \in \mathcal{A}_{0}(\aleph ; d, J)$, defined in Section 3.3.

Lemma 5.4 The bundle homomorphism

$$
\widetilde{\mathfrak{D}}_{0} \in \Gamma\left(\widetilde{\mathfrak{M}}_{0,(\aleph, J)}^{0}\left(\mathbb{P}^{n}, d\right) ; \operatorname{Hom}\left(\pi_{\mathbb{P} F_{(\aleph, J)}}^{*} \mathcal{V}_{(\aleph, J)}, \mathbb{E}_{0}^{*} \otimes \pi_{F_{(\aleph, J)}}^{*} \mathrm{ev}_{0}^{*} \mathcal{L}\right)\right)
$$

is surjective on the complement of the subvarieties $\widetilde{\mathfrak{M}}_{0, \varrho^{*}}^{0}\left(\mathbb{P}^{n}, d\right)$ with $\varrho^{*} \in \mathcal{A}_{0}(\boldsymbol{\aleph} ; d, J)$. Furthermore, for every $\varrho^{*} \in \mathcal{A}_{0}(\aleph ; d, J)$ as in Lemma 3.10, the differential of $\widetilde{\mathfrak{D}}_{0}$,

$$
\begin{aligned}
& \nabla \widetilde{\mathfrak{D}}_{0}: \mathcal{N}_{\iota_{0, \varrho^{*}}} \longrightarrow \iota_{0, \varrho^{*}}^{*} \operatorname{Hom}\left(\pi_{\mathbb{P} F_{(\aleph, J)}}^{*} \mathcal{V}_{(\aleph, J)}, \mathbb{E}_{0}^{*} \otimes \pi_{F_{(\aleph, J)}}^{*} \mathrm{ev}_{0}^{*} \mathcal{L}\right) \\
& =\pi_{P}^{*} \mathbb{E}_{0}^{*} \otimes \pi_{B}^{*} \operatorname{Hom}\left(\mathcal{V}_{\left(\aleph_{B}\left(\varrho^{*}\right), J_{B}\left(\varrho^{*}\right)\right)}, \mathrm{ev}_{0}^{*} \mathcal{L}\right),
\end{aligned}
$$

in the normal direction to the immersion $\iota_{0, \varrho^{*}}$ is given by

$$
\begin{aligned}
\left.\nabla \widetilde{\mathfrak{D}}_{0}\right|_{\pi_{P}^{*} L_{0,(l, i)} \otimes \pi_{B}^{*} \pi_{(l, i)}^{*} L_{0}} & =\pi_{P}^{*} s_{0,(l, i)} \otimes \pi_{B}^{*} \pi_{(l, i)}^{*} \mathfrak{D}_{0} \quad \forall i \in\left[m_{l}^{*}\right], l \in \mathfrak{\aleph}_{P}\left(\varrho^{*}\right), \\
\left.\nabla \widetilde{\mathfrak{D}}_{0}\right|_{\mathcal{N}_{\iota_{0, \varrho^{*}}^{\top}}^{\top}} & =\pi_{P}^{*} \operatorname{id} \otimes \pi_{B}^{*} \mathfrak{D}_{\left(\aleph_{B}\left(\varrho^{*}\right), J_{B}\left(\varrho^{*}\right)\right)}
\end{aligned}
$$


where $s_{0,(l, i)}$ is the homomorphism defined in Section 2.4.

This lemma follows immediately from Lemma 5.3.

Proposition 5.5 With notation as above, there exists a surjective bundle homomorphism

$\widetilde{\mathfrak{D}}_{(\aleph, J)} \in \Gamma\left(\widetilde{\mathfrak{M}}_{0,(\aleph, J)}\left(\mathbb{P}^{n}, d\right) ;\right.$

$$
\left.\operatorname{Hom}\left(\pi_{0,(\aleph, J)}^{*} \pi_{\mathbb{P} F_{(\aleph, J)}}^{*} \mathcal{V}_{(\aleph, J)}, \widetilde{\mathbb{E}}^{*} \otimes \pi_{0,(\aleph, J)}^{*} \pi_{\mathbb{P} F_{(\aleph, J)}}^{*} \mathrm{ev}_{0}^{*} \mathcal{L}\right)\right)
$$

such that

$$
\left.\widetilde{\mathfrak{D}}_{(\aleph, J)}\right|_{\mathbb{P} F_{(\aleph, J)}^{0}}=\left.\widetilde{\mathfrak{D}}_{0}\right|_{\mathbb{P} F_{(\aleph, J)}^{0}},
$$

where $\mathbb{P} F_{(\aleph, J)}^{0}=\mathbb{P} F_{(\aleph, J)}$

$$
-\bigcup_{\varrho \in \mathcal{A}_{0}(\aleph ; d, J)} \widetilde{\mathfrak{M}}_{0, \varrho}^{0}\left(\mathbb{P}^{n}, d\right) \subset \widetilde{\mathfrak{M}}_{0,(\aleph, J)}^{0}\left(\mathbb{P}^{n}, d\right), \widetilde{\mathfrak{M}}_{0,(\aleph, J)}\left(\mathbb{P}^{n}, d\right) .
$$

In fact, in the notation of Section 3.4, for every $\varrho \in\{0\} \sqcup \mathcal{A}_{0}(\aleph ; d, J)$ there exists a bundle homomorphism

$$
\widetilde{\mathfrak{D}}_{\varrho} \in \Gamma\left(\widetilde{\mathfrak{M}}_{0,(\aleph, J)}^{\varrho} ; \operatorname{Hom}\left(\pi_{\varrho}^{*} \pi_{\mathbb{P} F_{(\aleph, J)}}^{*} \mathcal{V}_{(\aleph, J)}, \mathbb{E}_{\varrho}^{*} \otimes \pi_{\varrho}^{*} \pi_{\mathbb{P} F_{(\aleph, J)}}^{*} e v_{0}^{*} \mathcal{L}\right)\right)
$$

such that

(i) the restrictions of $\widetilde{\mathfrak{D}}_{\varrho}$ and $\widetilde{\mathfrak{D}}_{0}$ to $\mathbb{P} F_{(\aleph, J)}^{0}$ agree;

(ii) $\widetilde{\mathfrak{D}}_{\varrho}$ is surjective outside of the subvarieties $\widetilde{\mathfrak{M}}_{0, \varrho^{*}}^{\varrho}$ with $\varrho^{*}>\varrho$;

(iii) $\widetilde{\mathfrak{D}}_{\varrho}$ vanishes identically on the subvarieties $\widetilde{\mathfrak{M}}_{0, \varrho^{*}}^{\varrho}$ with $\varrho^{*}>\varrho$;

(iv) for each $\varrho^{*}>\varrho$, the differential of $\widetilde{\mathfrak{D}}_{\varrho}$ in the normal direction to the immersion $\iota_{\varrho, \varrho^{*}}$ is given as in the statement of Lemma 5.4, but with $s_{0,(l, i)}$ replaced by $s_{\rho_{Q^{*}}(\varrho),(l, i)}$.

Similarly to the construction of the bundle sections $\widetilde{\mathcal{D}}_{\varrho}$ in Section 3.4 , we construct the bundle homomorphisms $\widetilde{\mathfrak{D}}_{\varrho}$ inductively starting with $\widetilde{\mathfrak{D}}_{0}$ and twisting by the exceptional divisor at each step. The inductive assumptions (i)-(iv) are analogous to (I3), (I4), and (I12) in Section 3.4 and are verified similarly. Of course, we take

$$
\widetilde{\mathfrak{D}}_{(\boldsymbol{\aleph}, J)}=\widetilde{\mathfrak{D}}_{\varrho_{\max }} .
$$




\subsection{Structure of the cone $\mathcal{V}_{1, k}^{d}$}

In this subsection we describe the structure of the cone

$$
p_{0}: \overline{\mathfrak{M}}_{1, k}(\mathcal{L}, d) \longrightarrow \overline{\mathfrak{M}}_{1, k}\left(\mathbb{P}^{n}, d\right)
$$

restating the primary structural result of [25].

For each element $\sigma=\left(m ; J_{P}, J_{B}\right)$ of $\mathcal{A}_{1}(d, k)$, let

$$
\mathcal{V}_{1, \sigma}^{0} \equiv \overline{\mathfrak{M}}_{1, \sigma}(\mathcal{L}, d)=p_{0}^{-1}\left(\overline{\mathfrak{M}}_{1, \sigma}^{0}\right) \subset \mathcal{V}_{1, k}^{0} \equiv \overline{\mathfrak{M}}_{1, k}(\mathcal{L}, d),
$$

with $\overline{\mathfrak{M}}_{1, \sigma}^{0}$ as in Equation (4-3). The subvarieties $\overline{\mathfrak{M}}_{1, \sigma}(\mathcal{L}, d)$ of $\overline{\mathfrak{M}}_{1, k}(\mathcal{L}, d)$ can also be defined analogously to the subvarieties $\overline{\mathfrak{M}}_{1, \sigma}\left(\mathbb{P}^{n}, d\right)$ of $\overline{\mathfrak{M}}_{1, k}\left(\mathbb{P}^{n}, d\right)$; see the beginning of Section 1.2. Similarly to Section 4.2, let

$$
j_{0, \sigma}: \overline{\mathcal{M}}_{1,\left(I_{P}(\sigma), J_{P}(\sigma)\right)}^{0} \times \mathcal{V}_{\left(\aleph_{B}(\sigma), J_{B}(\sigma)\right)} \longrightarrow \mathcal{V}_{1, \sigma}^{0} \subset \mathcal{V}_{1, k}^{0}
$$

be the natural node-identifying immersion so that the diagram

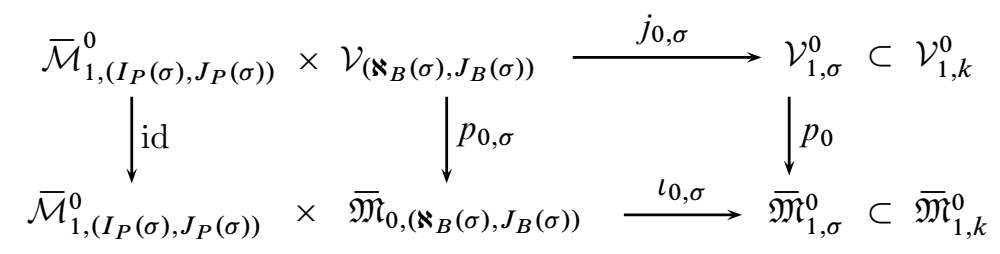

commutes.

Lemma 5.6 If $d, n \in \mathbb{Z}^{+}$and $k \in \overline{\mathbb{Z}}^{+}$, the collection $\left\{j_{0, \sigma}\right\}_{\sigma \in \mathcal{A}_{1}(d, k)}$ of immersions is properly self-intersecting. For every $\sigma \in \mathcal{A}_{1}(d, k)$,

$$
\mathcal{N}_{j_{0, \sigma}}^{\text {ide }}=\left\{\mathrm{id} \times p_{0, \sigma}\right\}^{*} \mathcal{N}_{l_{0, \sigma}}^{\text {ide }}
$$

is an idealized normal bundle for $j_{0, \sigma}$.

The differential $d p_{0}$ of $p_{0}$ induces a surjective linear map

$$
\operatorname{Im} d j_{0, \sigma} \longrightarrow \operatorname{Im} d \iota_{0, \sigma} .
$$

Since the fibers of $p_{0}$ are vector spaces, it follows that $d p_{0}$ induces an injection

$$
j_{0, \sigma}^{*} T C \mathcal{V}_{1, k}^{0} / \operatorname{Im} d j_{0, \sigma} \longrightarrow \iota_{0, \sigma}^{*} T C \overline{\mathfrak{M}}_{1, k}^{0} / \operatorname{Im} d \iota_{0, \sigma}
$$

Thus, Lemma 5.6 follows from Lemma 4.7. 
We denote by $\mathcal{V}_{1,(0)}^{0}$ the main component $\overline{\mathfrak{M}}_{1, k}^{0}(\mathcal{L}, d)$ of the moduli space $\overline{\mathfrak{M}}_{1, k}(\mathcal{L}, d)$. If $\sigma \in \mathcal{A}_{1}(d, k)$, we put

$$
\mathcal{W}_{\sigma}^{0}=j_{0, \sigma}^{-1}\left(\mathcal{V}_{1,(0)}^{0}\right) \equiv j_{0, \sigma}^{-1}\left(\mathcal{V}_{1,(0)}^{0} \cap \mathcal{V}_{1, \sigma}^{0}\right) .
$$

Note that

$$
\left\{\mathrm{id} \times p_{0, \sigma}\right\}\left(\mathcal{W}_{\sigma}^{0}\right)=\overline{\mathcal{Z}}_{\sigma}^{0} \equiv \iota_{0, \sigma}^{-1}\left(\overline{\mathfrak{M}}_{1,(0)}^{0}\right) .
$$

Let $\mathcal{N} \mathcal{W}_{\sigma}^{0} \subset \mathcal{N}_{j_{0, \sigma}}^{\text {ide }}$ be the normal cone $\mathcal{N}_{j_{0, \sigma} \mid \mathcal{V}_{1,(0)}^{0}}$ for $\left.j_{0, \sigma}\right|_{\mathcal{W}_{\sigma}^{0}}$ in $\mathcal{V}_{1,(0)}^{0}$. Its structure is described in Lemma 5.7 below. Let $\mathfrak{D}_{0, \sigma}$ be the section of the bundle

$$
\begin{aligned}
\operatorname{Hom}\left(\pi_{B}^{*} \mathcal{V}_{\left(\aleph_{B}(\sigma), J_{B}(\sigma)\right)}, \operatorname{Hom}\left(\mathcal{N}_{\iota_{0, \sigma}}^{\text {ide }}, \pi_{P}^{*} \mathbb{E}_{0}^{*} \otimes \pi_{B}^{*} \operatorname{ev}_{0}^{*} \mathcal{L}\right)\right) \\
=\operatorname{Hom}\left(\mathcal{N}_{\iota_{0, \sigma}}^{\text {ide }}, \pi_{P}^{*} \mathbb{E}_{0}^{*} \otimes \pi_{B}^{*} \operatorname{Hom}\left(\mathcal{V}_{\left(\aleph_{B}(\sigma), J_{B}(\sigma)\right)}, \mathrm{ev}_{0}^{*} \mathcal{L}\right)\right)
\end{aligned}
$$

over $\overline{\mathcal{M}}_{1,\left(I_{P}(\sigma), J_{P}(\sigma)\right)}^{0} \times \overline{\mathfrak{M}}_{0,\left(\aleph_{B}(\sigma), J_{B}(\sigma)\right)}$ defined by

$$
\left.\mathfrak{D}_{0, \sigma}\right|_{\pi_{P}^{*} L_{i} \otimes \pi_{B}^{*} \pi_{i}^{*} L_{0}}=\pi_{P}^{*} s_{i} \otimes \pi_{B}^{*} \pi_{i}^{*} \mathfrak{D}_{0}, \quad \forall i \in[m],
$$

where $s_{i}$ and $\mathfrak{D}_{0}$ are as in Sections 2.2 and 5.2, respectively. If $\xi \in \pi_{B}^{*} \mathcal{V}_{\left(\aleph_{B}(\sigma), J_{B}(\sigma)\right)}$, we will view $\mathfrak{D}_{0, \sigma} \xi$ as a homomorphism

$$
\mathfrak{D}_{0, \sigma} \xi:\left.\mathcal{N}_{j_{0, \sigma}}^{\text {ide }}\right|_{\xi}=\left.\left.\mathcal{N}_{l_{0, \sigma}}^{\text {ide }}\right|_{\left\{\mathrm{id} \times p_{0, \sigma}\right\}(\xi)} \longrightarrow \pi_{P}^{*} \mathbb{E}_{0}^{*} \otimes \pi_{B}^{*} \mathrm{ev}_{0}^{*} \mathcal{L}\right|_{\left\{\mathrm{id} \times p_{0, \sigma}\right\}(\xi)} \text {. }
$$

Lemma 5.7 For all $\sigma \in \mathcal{A}_{1}(d, k), \mathcal{V}_{1,(0)}^{0}$ is a proper subvariety of $\mathcal{V}_{1, k}^{0}$ relative to the immersion $j_{0, \sigma}$. The homomorphism

$$
\mathcal{N} \mathcal{W}_{\sigma}^{0} \longrightarrow\left\{\mathrm{id} \times p_{0, \sigma}\right\}^{*} \mathcal{N} \overline{\mathcal{Z}}_{\sigma}^{0}
$$

induced by $d p_{0}$ is injective. Furthermore,

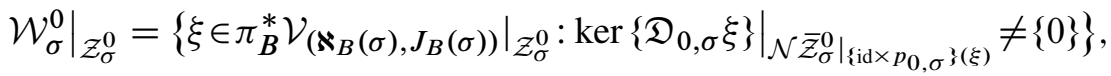

$$
\begin{aligned}
& \left.\mathcal{N} \mathcal{W}_{\sigma}^{0}\right|_{\xi}=\left.\left.\operatorname{ker}\left\{\mathfrak{D}_{0, \sigma} \xi\right\}\right|_{\left.\mathcal{N} \overline{\mathcal{Z}}_{\sigma}^{0}\right|_{\left\{\text {id } \times p_{0, \sigma}\right\}(\xi)}} \subset \mathcal{N}_{j_{0, \sigma}}^{\text {ide }} \quad \forall \xi \in \mathcal{W}_{\sigma}^{0}\right|_{\mathcal{Z}_{\sigma}^{0}} \text {. }
\end{aligned}
$$

Finally, $\mathcal{W}_{\sigma}^{0}$ is the closure of $\left.\mathcal{W}_{\sigma}^{0}\right|_{\mathcal{Z}_{\sigma}^{0}}$ in

$$
\overline{\mathcal{M}}_{1,\left(I_{P}(\sigma), J_{P}(\sigma)\right)}^{0} \times \mathcal{V}_{\left(\aleph_{B}(\sigma), J_{B}(\sigma)\right)}
$$

and $\mathcal{N} \mathcal{W}_{\sigma}^{0}$ is the closure of $\left.\mathcal{N} \mathcal{W}_{\sigma}^{0}\right|_{\left.\mathcal{W}_{\sigma}^{0}\right|_{\mathcal{Z}_{\sigma}^{0}}}$ in $\mathcal{N}_{j_{0, \sigma}}^{\text {ide }}$.

Since the fibers of $p_{0}$ are vector spaces, the first two sentences of this lemma follow from Lemma 4.10. The middle claim of Lemma 5.7 is a restatement of [25, Lemma 3.4]. The remaining claims of the lemma follow from [25, Lemma 3.4] by dimension counting, similarly to the argument following Lemma 4.10. 
Remark It may appear that the statement of Lemma 5.7 depends on the choice of a hermitian connection (or metric) in the line bundle $\mathcal{L} \longrightarrow \mathbb{P}^{n}$. As explained in detail in [25, Subsection 3.3], the dependence is only on the holomorphic structure of $\mathcal{L}$, as the case should be.

\subsection{Desingularization construction}

In this subsection we lift the inductive blowup construction of Section 4.3 to the cone

$$
p_{0}: \mathcal{V}_{1, k}^{0} \longrightarrow \overline{\mathfrak{M}}_{1, k}^{0} \text {. }
$$

For each $\sigma \in \mathcal{A}_{1}(d, k)$, let $\mathcal{V}_{1, k}^{\sigma} \equiv \pi_{\sigma}^{*} \mathcal{V}_{1, k}^{0}$ be the pullback of $\mathcal{V}_{1, k}^{0}$ to $\overline{\mathfrak{M}}_{1, k}^{\sigma}$ :

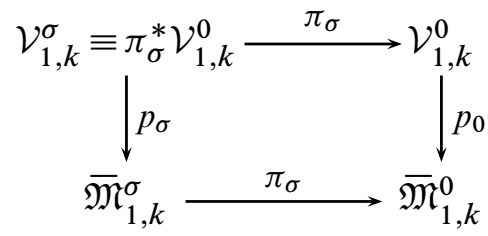

For each $\sigma^{\prime} \in \mathcal{A}_{1}(d, k)$, let

$$
\mathcal{V}_{1, \sigma^{\prime}}^{\sigma}=\left.\mathcal{V}_{1, k}^{\sigma}\right|_{\overline{\mathfrak{M}}_{1, \sigma^{\prime}}^{\sigma}}=\pi_{\sigma}^{-1}\left(\left.\mathcal{V}_{1, k}^{0}\right|_{\overline{\mathfrak{M}}_{1, \sigma^{\prime}}^{0}}\right) \text {. }
$$

The bundle homomorphisms $j_{0, \sigma^{\prime}}$ lift to bundle homomorphisms onto $\mathcal{V}_{1, \sigma^{\prime}}^{\sigma}$ covering the immersion $\iota_{\sigma, \sigma^{\prime}}$ of Section 4.3:

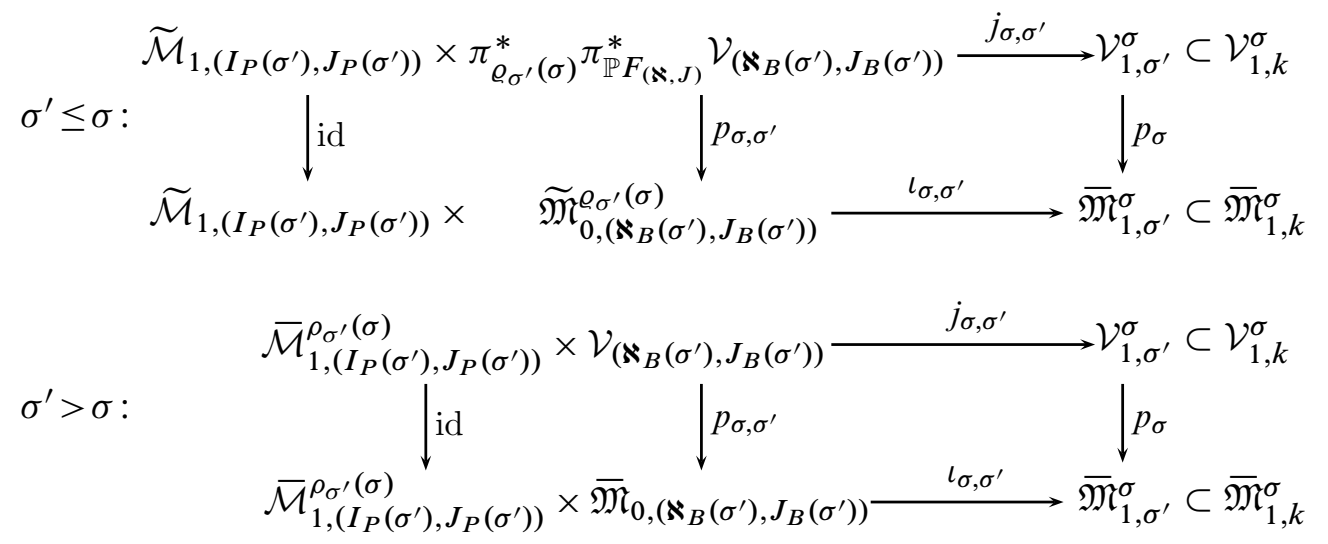

The collection $\left\{\iota_{\sigma, \sigma^{\prime}}\right\}_{\sigma^{\prime} \in \mathcal{A}_{1}(d, k)}$ of immersions is properly self-intersecting by the inductive assumption (I14) of Section 4.3. Thus, by the same argument as in the paragraph following Lemma 5.6, so is the collection $\left\{j_{\sigma, \sigma^{\prime}}\right\}_{\sigma^{\prime} \in \mathcal{A}_{1}(d, k)}$. Furthermore,

$$
\mathcal{N}_{j_{\sigma, \sigma^{\prime}}}^{\text {ide }}=\left\{\mathrm{id} \times p_{\sigma, \sigma^{\prime}}\right\}^{*} \mathcal{N}_{\iota_{\sigma, \sigma^{\prime}}}^{\text {ide }}
$$


is an idealized normal bundle for $j_{\sigma, \sigma^{\prime}}$. These two observations also follow from Lemma 5.6 by induction using Lemmas 4.2 and 4.3 .

Lemma 5.8 If $\sigma \in \mathcal{A}_{1}(d, k), \mathcal{V}_{1, \sigma}^{\sigma-1}$ is a smooth subvariety of $\mathcal{V}_{1, k}^{\sigma-1}$ and

$$
p_{\sigma}: \mathcal{V}_{1, k}^{\sigma} \longrightarrow \overline{\mathfrak{M}}_{1, k}^{\sigma}
$$

is the idealized blowup of $\mathcal{V}_{1, k}^{\sigma-1}$ along $\mathcal{V}_{1, \sigma}^{\sigma-1}$.

Recall from Section 4.3 that the immersion

$$
\bar{\iota}_{\sigma-1, \sigma}:\left(\widetilde{\mathcal{M}}_{1,\left(I_{P}(\sigma), J_{P}(\sigma)\right)} \times \overline{\mathfrak{M}}_{0,\left(\aleph_{B}(\sigma), J_{B}(\sigma)\right)}\right) / G_{\sigma} \longrightarrow \overline{\mathfrak{M}}_{1, \sigma}^{\sigma-1} \subset \overline{\mathfrak{M}}_{1, k}^{\sigma-1}
$$

induced by $\iota_{\sigma-1, \sigma}$ is an embedding and

$$
\tilde{\pi}_{\sigma}: \overline{\mathfrak{M}}_{1, k}^{\sigma} \longrightarrow \overline{\mathfrak{M}}_{1, k}^{\sigma-1}
$$

is the idealized blowup along $\overline{\mathfrak{M}}_{1, \sigma}^{\sigma-1}$. Thus, the immersion

$$
\bar{j}_{\sigma-1, \sigma}:\left(\widetilde{\mathcal{M}}_{1,\left(I_{P}(\sigma), J_{P}(\sigma)\right)} \times \mathcal{V}_{\left(\aleph_{B}(\sigma), J_{B}(\sigma)\right)}\right) / G_{\sigma} \longrightarrow \mathcal{V}_{1, \sigma}^{\sigma-1} \subset \mathcal{V}_{1, k}^{\sigma-1}
$$

induced by $j_{\sigma-1, \sigma}$ is also an embedding and $\mathcal{V}_{1, \sigma}^{\sigma-1}$ is a smooth subvariety of $\mathcal{V}_{1, k}^{\sigma-1}$.

Let $\quad \tilde{\pi}_{\sigma}: \mathcal{V} \longrightarrow \mathcal{V}_{1, k}^{\sigma-1}$

be the idealized blowup along $\mathcal{V}_{1, k}^{\sigma-1}$. Since

$$
\mathcal{N}_{j_{\sigma-1, \sigma}}^{\text {ide }}=\left\{\mathrm{id} \times p_{\sigma-1, \sigma}\right\}^{*} \mathcal{N}_{l_{\sigma-1, \sigma}}^{\text {ide }}
$$

and the linear map

$$
j_{\sigma-1, \sigma}^{*} T C \mathcal{V}_{1, k}^{\sigma-1} / \operatorname{Im} d j_{\sigma-1, \sigma} \longrightarrow \iota_{\sigma-1, \sigma}^{*} T C \overline{\mathfrak{M}}_{1, k}^{\sigma-1} / \operatorname{Im} d \iota_{\sigma-1, \sigma}
$$

induced by $d p_{\sigma-1}$ is injective, $p_{\sigma-1}$ lifts to a map $p$ over the blowdown maps $\tilde{\pi}_{\sigma}$ :

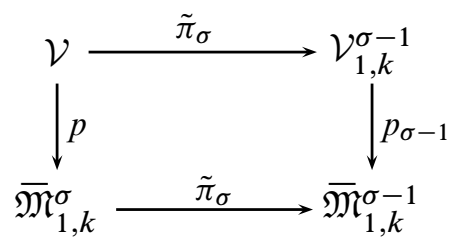

Then $p$ and the top arrow $\tilde{\pi}_{\sigma}$ factor through a morphism $f$ to $\tilde{\pi}_{\sigma}^{*} \mathcal{V}_{1, k}^{\sigma-1}$ : 


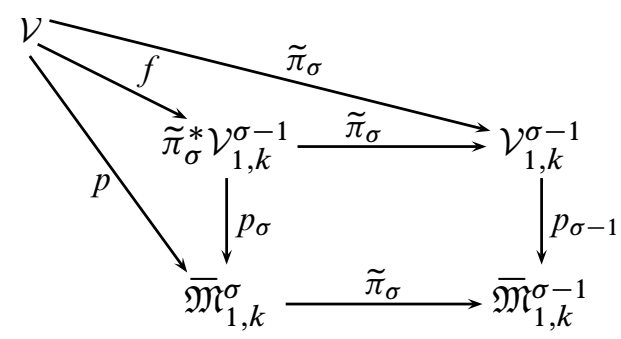

We show in the next paragraph that $f$ is an isomorphism. Since $\tilde{\pi}_{\sigma}^{*} \mathcal{V}_{1, k}^{\sigma-1}=\mathcal{V}_{1, k}^{\sigma}$, this implies the second statement of Lemma 5.8.

By construction, the maps

$$
\tilde{\pi}_{\sigma}: \overline{\mathfrak{M}}_{1, k}^{\sigma} \longrightarrow \overline{\mathfrak{M}}_{1, k}^{\sigma-1} \quad \text { and } \quad \tilde{\pi}_{\sigma}: \mathcal{V} \longrightarrow \mathcal{V}_{1, k}^{\sigma-1}
$$

are isomorphisms on the complements of the idealized exceptional divisors

$$
\overline{\mathfrak{M}}_{1, \sigma}^{\sigma} \equiv \mathcal{E}_{\overline{\mathfrak{M}}_{1, \sigma}^{\sigma-1}}^{\text {ide }} \subset \overline{\mathfrak{M}}_{1, k}^{\sigma} \quad \text { and } \quad \mathcal{V}_{1, \sigma}^{\sigma} \equiv \mathcal{E}_{\mathcal{V}_{1, \sigma}^{\sigma-1}}^{\text {ide }} \subset \mathcal{V}
$$

Thus, $f: \mathcal{V} \longrightarrow \tilde{\pi}_{\sigma}^{*} \mathcal{V}_{1, k}$ is an isomorphism over the complement of $\overline{\mathfrak{M}}_{1, \sigma}^{\sigma}$ in $\overline{\mathfrak{M}}_{1, k}^{\sigma}$. In particular, $f$ is linear on all fibers of $p$. Furthermore,

$$
\left.\tilde{\pi}_{\sigma}^{*} \mathcal{V}_{1, k}^{\sigma-1}\right|_{\overline{\mathfrak{M}}_{1, \sigma}^{\sigma}}=\left\{(\ell, v) \in \mathbb{P} \mathcal{N}_{\overline{\mathfrak{M}}_{1, \sigma}^{\sigma-1}}^{\mathrm{ide}} \times \mathcal{V}_{1, k}^{\sigma-1}: \tilde{\pi}_{\sigma}(\ell)=p_{\sigma-1}(v)\right\}
$$

On the other hand, since

$$
\mathcal{N}_{\mathcal{V}_{1, \sigma}^{\sigma-1}}^{\text {ide }}=p_{\sigma-1}^{*} \mathcal{N}_{\overline{\mathfrak{M}}_{1, \sigma}^{\sigma-1}}^{\text {ide }}
$$

by $(5-1)$, we have

$$
\mathcal{V}_{1, \sigma}^{\sigma}=p_{\sigma-1}^{*} \mathbb{P} \mathcal{N}_{\mathfrak{M}_{1, \sigma}^{\sigma-1}}^{\text {ide }}=\left\{(v, \ell) \in \mathcal{V}_{1, k}^{\sigma-1} \times \mathbb{P} \mathcal{N}_{\overline{\mathfrak{M}}_{1, \sigma}^{\sigma-1}}^{\text {ide }}: p_{\sigma-1}(v)=\tilde{\pi}_{\sigma}(\ell)\right\} .
$$

Thus, the restriction of $f$ to $\mathcal{V}_{1, \sigma}^{\sigma}$ must interchange $v$ and $\ell$, ie it is a vector bundle isomorphism over $\overline{\mathfrak{M}}_{1, k}^{\sigma}$. Finally, $\overline{\mathfrak{M}}_{1, \sigma}^{\sigma-1}$ is a smooth subvariety of $\mathcal{V}_{1, k}^{\sigma-1}$ and

$$
T\left(\overline{\mathfrak{M}}_{1, k}^{\sigma-1} \cap \mathcal{V}_{1, \sigma}^{\sigma-1}\right)=T \overline{\mathfrak{M}}_{1, \sigma}^{\sigma-1}=T C \overline{\mathfrak{M}}_{1, k}^{\sigma-1} \cap T \mathcal{V}_{1, \sigma}^{\sigma-1} \subset T C \mathcal{V}_{1, k}^{\sigma-1}
$$

Thus, similarly to (1) of Lemma 3.3, the proper transform of $\overline{\mathfrak{M}}_{1, k}^{\sigma-1}$ in $\mathcal{V}$ is the blowup of $\overline{\mathfrak{M}}_{1, k}^{\sigma-1}$ along

$$
\overline{\mathfrak{M}}_{1, k}^{\sigma-1} \cap \mathcal{V}_{1, \sigma}^{\sigma-1}=\overline{\mathfrak{M}}_{1, \sigma}^{\sigma-1}
$$

ie $\mathcal{V}$ contains $\overline{\mathfrak{M}}_{1, k}^{\sigma}$ as the zero section. The map $f$ must be the identity on $\overline{\mathfrak{M}}_{1, k}^{\sigma}$. Since $f$ is a linear isomorphism on all fibers of $p$ by the above, it then follows that $f$ is an isomorphism everywhere. 
Remark If $\mathcal{V}_{1, k}^{\sigma-1}$ is a vector bundle over $\overline{\mathfrak{M}}_{1, k}^{\sigma-1}$, the second statement of Lemma 5.8 applies to standard blowups of $\overline{\mathfrak{M}}_{1, k}^{\sigma-1}$ and $\mathcal{V}_{1, k}^{\sigma-\hat{1}}$ as well. However, the second statement does not generally apply to standard blowups in the setting of Lemma 5.8, as the analogue of the morphism $f$ may not be surjective.

By the inductive assumption ( $I 1)$ of Section 4.3, the projection map $\pi_{\sigma}$ is an isomorphism outside of the subvarieties $\mathcal{V}_{1, \sigma^{\prime}}^{\sigma}$ with $\sigma^{\prime} \leq \sigma$. We denote by

$$
\mathcal{V}_{1,(0)}^{\sigma} \subset \mathcal{V}_{1, k}^{\sigma}
$$

the proper transform of $\mathcal{V}_{1,(0)}^{0}$. For each $\sigma^{\prime} \in \mathcal{A}_{1}(d, k)$, let

$$
\mathcal{W}_{\sigma^{\prime}}^{\sigma}=j_{\sigma, \sigma^{\prime}}^{-1}\left(\mathcal{V}_{1,(0)}^{\sigma}\right)=j_{\sigma, \sigma^{\prime}}^{-1}\left(\mathcal{V}_{1,(0)}^{\sigma} \cap \mathcal{V}_{1, \sigma^{\prime}}^{\sigma}\right)
$$

By the inductive assumption (I15) of Section 4.3, $\overline{\mathfrak{M}}_{1,(0)}^{\sigma}$ is a proper subvariety of $\overline{\mathfrak{M}}_{1, k}^{\sigma}$ with respect to the immersion $\iota_{\sigma, \sigma^{\prime}}$. Thus, by the same argument as in the paragraph following Lemma 5.7, the subvariety $\mathcal{V}_{1,(0)}^{\sigma}$ of $\mathcal{V}_{1, k}^{\sigma}$ is proper with respect to the immersion $j_{\sigma, \sigma^{\prime}}$. Furthermore, if

$$
\mathcal{N} \mathcal{W}_{\sigma^{\prime}}^{\sigma} \equiv \mathcal{N} \mathcal{W}_{j_{\sigma, \sigma^{\prime}} \mid \mathcal{V}_{1,(0)}^{\sigma}} \subset \mathcal{N}_{j_{\sigma^{\prime}, \sigma}^{\text {ide }}}
$$

denotes the normal cone for $\left.j_{\sigma^{\prime}, \sigma}\right|_{\mathcal{W}_{\sigma^{\prime}}^{\sigma}}$ in $\mathcal{V}_{1,(0)}^{\sigma}$, then the homomorphism

$$
\mathcal{N} \mathcal{W}_{\sigma^{\prime}}^{\sigma} \longrightarrow\left\{\operatorname{id} \times p_{\sigma, \sigma^{\prime}}\right\}^{*} \mathcal{N} \overline{\mathcal{Z}}_{\sigma^{\prime}}^{\sigma}
$$

induced by $d p_{\sigma}$ is injective. These two observations also follow from Lemma 5.7 by induction using Lemma 4.5.

If $\sigma^{\prime} \in \mathcal{A}_{1}(d, k)$, let

$$
\widetilde{\mathcal{Z}}_{\sigma^{\prime} ; B}^{0}=\left.\widetilde{\mathcal{D}}_{0}^{-1}(0) \cap \mathbb{P} F_{\left(I_{P}\left(\sigma^{\prime}\right), J_{P}\left(\sigma^{\prime}\right)\right)}\right|_{\mathfrak{M}_{0,\left(\aleph_{B}(\sigma), J_{B}(\sigma)\right)}} .
$$

By the inductive assumptions (I7) in Section 4.3 and (I4) in Section 3.4,

$$
\overline{\mathcal{Z}}_{\sigma^{\prime}}^{\sigma} \equiv \iota_{\sigma, \sigma^{\prime}}^{-1}\left(\overline{\mathfrak{M}}_{1,(0)}^{\sigma}\right)
$$

is the closure of $\widetilde{\mathcal{M}}_{1,\left(I_{P}\left(\sigma^{\prime}\right), J_{P}\left(\sigma^{\prime}\right)\right)} \times \widetilde{\mathcal{Z}}_{\sigma^{\prime} ; B}^{0}$ in

$$
\widetilde{\mathcal{M}}_{1,\left(I_{P}\left(\sigma^{\prime}\right), J_{P}\left(\sigma^{\prime}\right)\right)} \times \widetilde{\mathfrak{M}}_{0,\left(\aleph_{B}\left(\sigma^{\prime}\right), J_{B}\left(\sigma^{\prime}\right)\right)}^{\varrho}
$$

for all $\sigma \in \mathcal{A}_{1}(k, d)$ such that $\sigma^{\prime} \leq \sigma$.

Suppose $\sigma \in\{0\} \cup \mathcal{A}_{1}(d, k)$ and $\sigma^{\prime} \in \mathcal{A}_{1}(d, k)$. We claim that 
(I1) if $\sigma^{\prime} \leq \sigma$, then $\mathcal{W}_{\sigma^{\prime}}^{\sigma}$ is the closure of

$$
\begin{aligned}
\widetilde{\mathcal{M}}_{1,\left(I_{P}\left(\sigma^{\prime}\right), J_{P}\left(\sigma^{\prime}\right)\right)} \times \operatorname{ker} & \left.\widetilde{\mathfrak{D}}_{0}\right|_{\widetilde{\mathcal{Z}}_{\sigma^{\prime} ; B}^{0}} \\
\subset & \widetilde{\mathcal{M}}_{1,\left(I_{P}\left(\sigma^{\prime}\right), J_{P}\left(\sigma^{\prime}\right)\right)} \times \pi_{\mathbb{P} F_{(\aleph, J)}}^{*} \mathcal{V}_{\left(\aleph_{B}\left(\sigma^{\prime}\right), J_{B}\left(\sigma^{\prime}\right)\right)}, \\
& \widetilde{\mathcal{M}}_{1,\left(I_{P}\left(\sigma^{\prime}\right), J_{P}\left(\sigma^{\prime}\right)\right)} \times \pi_{\varrho_{\sigma^{\prime}}(\sigma)}^{*} \pi_{\mathbb{P} F_{(\aleph, J)}}^{*} \mathcal{V}_{\left(\aleph_{B}\left(\sigma^{\prime}\right), J_{B}\left(\sigma^{\prime}\right)\right)}
\end{aligned}
$$

in $\widetilde{\mathcal{M}}_{1,\left(I_{P}\left(\sigma^{\prime}\right), J_{P}\left(\sigma^{\prime}\right)\right)} \times \pi_{\varrho_{\sigma^{\prime}}(\sigma)}^{*} \pi_{\mathbb{P} F_{(\aleph, J)}}^{*} \mathcal{V}_{\left(\aleph_{B}\left(\sigma^{\prime}\right), J_{B}\left(\sigma^{\prime}\right)\right)}$ and

$$
\mathcal{N} \mathcal{W}_{\sigma^{\prime}}^{\sigma}=\left.\mathcal{N}_{j_{\sigma, \sigma^{\prime}}}^{\text {ide }}\right|_{\mathcal{W}_{\sigma^{\prime}}^{\sigma}}
$$

(I2) if $\sigma^{\prime}>\sigma$, then $\mathcal{W}_{\sigma^{\prime}}^{\sigma}$ and $\mathcal{N} \mathcal{W}_{\sigma^{\prime}}^{\sigma}$ are the closures of

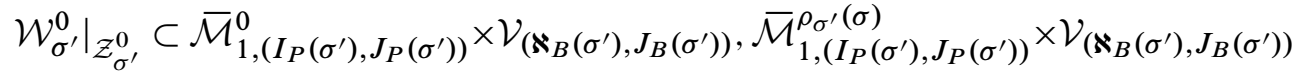

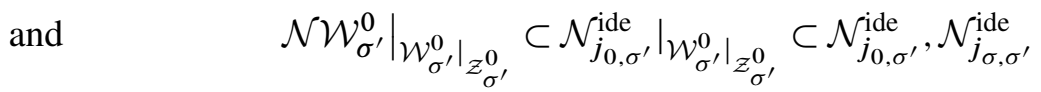

in $\overline{\mathcal{M}}_{1,\left(I_{P}\left(\sigma^{\prime}\right), J_{P}\left(\sigma^{\prime}\right)\right)}^{\rho_{\sigma^{\prime}}(\sigma)} \times \mathcal{V}_{\left(\aleph_{B}\left(\sigma^{\prime}\right), J_{B}\left(\sigma^{\prime}\right)\right)}$ and in $\mathcal{N}_{j_{\sigma, \sigma^{\prime}}}^{\text {ide }}$, respectively.

If $\sigma=0$, the assumption (I1) is trivially satisfied, while (I2) constitutes part of Lemma 5.7. Suppose $\sigma \in \mathcal{A}_{1}(d, k)$ and the two assumptions hold with $\sigma$ replaced by $\sigma-1$. By Lemma 5.8, $\mathcal{V}_{1, k}^{\sigma}$ is the idealized blowup of $\mathcal{V}_{1, k}^{\sigma-1}$ along $\mathcal{V}_{1, \sigma}^{\sigma-1}$. Thus, by the last statement of Lemma 4.5 both of the inductive assumptions continue to hold for $\sigma^{\prime} \neq \sigma$.

On the other hand, let

$$
\begin{aligned}
\mathcal{Z}_{\sigma ; B} & =\left\{b \in \mathfrak{M}_{0,\left(\aleph_{B}(\sigma), J_{B}(\sigma)\right)}: \operatorname{ker} \mathcal{D}_{\left(\aleph_{B}(\sigma), J_{B}(\sigma)\right)} \neq 0\right\}, \\
\mathcal{W}_{\sigma ; B}^{0} & =\left\{\xi \in \mathcal{V}_{\left(\aleph_{B}(\sigma), J_{B}(\sigma)\right)} \mid \mathcal{Z}_{\sigma ; B}:\right. \\
\left.\left.\operatorname{ker}\left\{\mathfrak{D}_{\left(\aleph_{B}(\sigma), J_{B}(\sigma)\right)} \xi\right\}\right|_{\left.\operatorname{ker} \mathcal{D}_{\left(\aleph_{B}(\sigma), J_{B}(\sigma)\right)}\right|_{p_{\sigma-1, \sigma}(\xi)}} \neq\{0\}\right\}, & \\
\mathcal{N} \mathcal{W}_{\sigma ; B}^{0}=\left\{(\xi, v): \xi \in \mathcal{W}_{\sigma ; B}^{0},\left.v \in \operatorname{ker}\left\{\mathfrak{D}_{0, \sigma} \xi\right\}\right|_{\left.\left.\operatorname{ker} \mathcal{D}_{\left(\aleph_{B}(\sigma), J_{B}(\sigma)\right)}\right|_{p_{\sigma-1, \sigma}(\xi)}\right\}}\right. & \subset p_{\sigma-1, \sigma}^{*} F_{\left(\aleph_{B}(\sigma), J_{B}(\sigma)\right) .}
\end{aligned}
$$

By the inductive assumption (I12) in Section 4.3,

$$
\begin{gathered}
\overline{\mathcal{Z}}_{\sigma}^{\sigma-1} \cap\left(\widetilde{\mathcal{M}}_{1,\left(I_{P}(\sigma), J_{P}(\sigma)\right)} \times \mathfrak{M}_{0,\left(\aleph_{B}(\sigma), J_{B}(\sigma)\right)}\right)=\widetilde{\mathcal{M}}_{1,\left(I_{P}(\sigma), J_{P}(\sigma)\right)} \times \mathcal{Z}_{\sigma ; B}, \\
\left.\mathcal{N} \overline{\mathcal{Z}}_{\sigma}^{0}\right|_{\widetilde{\mathcal{M}}_{1,\left(I_{P}(\sigma), J_{P}(\sigma)\right)} \times \mathcal{Z}_{\sigma ; B}}=\pi_{P}^{*} \mathbb{L} \otimes \pi_{B}^{*} \operatorname{ker} \mathcal{D}_{\left(\aleph_{B}(\sigma), J_{B}(\sigma)\right)} .
\end{gathered}
$$


By the inductive assumption (I2) above, Lemma 5.7, and the inductive assumption (I11) in Section 4.3, $\mathcal{W}_{\sigma}^{\sigma-1}$ and $\mathcal{N} \mathcal{W}_{\sigma}^{\sigma-1}$ are the closures of

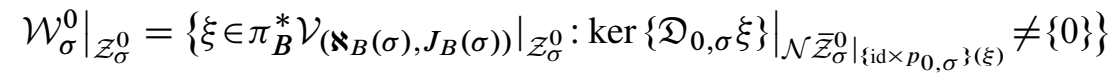

$$
\begin{aligned}
& \text { and }\left.\quad \mathcal{N} \mathcal{W}_{\sigma}^{0}\right|_{\left.\mathcal{W}_{\sigma}^{0}\right|_{\mathcal{Z}_{\sigma}^{0}}}=\left\{(\xi, v):\left.\xi \in \mathcal{W}_{\sigma}^{0}\right|_{\mathcal{Z}_{\sigma}^{0}},\left.v \in \operatorname{ker}\left\{\mathfrak{D}_{0, \sigma} \xi\right\}\right|_{\left.\mathcal{N} \overline{\mathcal{Z}}_{\sigma}^{0}\right|_{\left\{\mathrm{id} \times p_{0, \sigma}\right\}(\xi)}}\right\} \\
& \subset \mathcal{N}_{j_{0, \sigma}}^{\text {ide }}, \mathcal{N}_{j_{\sigma-1, \sigma}}^{\text {ide }}
\end{aligned}
$$

in $\widetilde{\mathcal{M}}_{1,\left(I_{P}(\sigma), J_{P}(\sigma)\right)} \times \mathcal{V}_{\left(\aleph_{B}(\sigma), J_{B}(\sigma)\right)}$ and in

$$
\mathcal{N}_{j_{\sigma-1, \sigma}}^{\text {ide }}=\pi_{P}^{*} \mathbb{L} \otimes \pi_{B}^{*} p_{\sigma-1, \sigma}^{*} F_{\left(\aleph_{B}(\sigma), J_{B}(\sigma)\right)} .
$$

As before,

$$
\pi_{P}, \pi_{B}: \widetilde{\mathcal{M}}_{1,\left(I_{P}(\sigma), J_{P}(\sigma)\right)} \times \mathcal{V}_{\left(\aleph_{B}(\sigma), J_{B}(\sigma)\right)} \longrightarrow \widetilde{\mathcal{M}}_{1,\left(I_{P}(\sigma), J_{P}(\sigma)\right)}, \mathcal{V}_{\left(\aleph_{B}(\sigma), J_{B}(\sigma)\right)}
$$

are the projections onto the principle and bubble components. The bundle homomorphisms $s_{i}$ and $\tilde{s}_{i}$ of Section 2.3 agree on

$$
\mathcal{M}_{1,\left(I_{P}(\sigma), J_{P}(\sigma)\right)} \subset \overline{\mathcal{M}}_{1,\left(I_{P}(\sigma), J_{P}(\sigma)\right)}, \widetilde{\mathcal{M}}_{1,\left(I_{P}(\sigma), J_{P}(\sigma)\right)} .
$$

The homomorphism $\tilde{s}_{i}$ is an isomorphism from $\tilde{L}_{i}$ to $\widetilde{\mathbb{E}}^{*}$ over $\widetilde{\mathcal{M}}_{1,\left(I_{P}(\sigma), J_{P}(\sigma)\right)}$, and both line bundles are isomorphic to $\mathbb{L}$. It follows that $\mathcal{W}_{\sigma}^{\sigma-1}$ and $\mathcal{N} \mathcal{W}_{\sigma}^{\sigma-1}$ are the closures of

$$
\widetilde{\mathcal{M}}_{1,\left(I_{P}(\sigma), J_{P}(\sigma)\right)} \times \mathcal{W}_{\sigma ; B}^{0} \quad \text { and } \quad \pi_{P}^{*} \mathbb{L} \otimes \pi_{B}^{*} \mathcal{N} \mathcal{W}_{\sigma ; B}^{0}
$$

in $\widetilde{\mathcal{M}}_{1,\left(I_{P}(\sigma), J_{P}(\sigma)\right)} \times \mathcal{V}_{\left(\aleph_{B}(\sigma), J_{B}(\sigma)\right)}$ and in

$$
\mathcal{N}_{j_{\sigma-1, \sigma}}^{\text {ide }}=\pi_{P}^{*} \mathbb{L} \otimes \pi_{B}^{*} p_{\sigma-1, \sigma}^{*} F_{\left(\aleph_{B}(\sigma), J_{B}(\sigma)\right)} .
$$

Thus, by the first statement of Lemma 4.5 ,

$$
\mathcal{W}_{\sigma}^{\sigma} \equiv j_{\sigma, \sigma}^{-1}\left(\mathcal{V}_{1,(0)}^{\sigma}\right)
$$

is the closure of

$$
\begin{aligned}
\mathbb{P}\left(\pi_{P}^{*} \mathbb{L} \otimes \pi_{B}^{*} \mathcal{N} \mathcal{W}_{\sigma ; B}^{0}\right)= & \widetilde{\mathcal{M}}_{1,\left(I_{P}(\sigma), J_{P}(\sigma)\right)} \times \mathbb{P} \mathcal{N} \mathcal{W}_{\sigma ; B}^{0} \\
= & \widetilde{\mathcal{M}}_{1,\left(I_{P}(\sigma), J_{P}(\sigma)\right)} \times\left.\operatorname{ker} \widetilde{\mathfrak{D}}_{0}\right|_{\widetilde{\mathcal{Z}}_{\sigma ; B}^{0}} \\
& \subset \mathbb{P} \mathcal{N}_{j_{\sigma-1, \sigma}}^{\text {ide }}=\widetilde{\mathcal{M}}_{1,\left(I_{P}(\sigma), J_{P}(\sigma)\right)} \times \pi_{\mathbb{P} F_{(\aleph, J)}}^{*} \mathcal{V}_{\left(\aleph_{B}(\sigma), J_{B}(\sigma)\right)},
\end{aligned}
$$

ie the first part of the inductive assumption (I1) for $\sigma^{\prime}=\sigma$ is satisfied. Furthermore, by the second part of (1) of Lemma 4.5,

$$
\mathcal{N} \mathcal{W}_{\sigma}^{\sigma}=\left.\gamma_{\mathcal{V}_{1, \sigma}^{\sigma-1}}\right|_{\mathcal{W}_{\sigma}^{\sigma}}=\left.\left\{\mathrm{id} \times p_{\sigma, \sigma}\right\}^{*} \gamma_{\overline{\mathfrak{M}}_{1, \sigma}^{\sigma-1}}\right|_{\mathcal{W}_{\sigma}^{\sigma}}=\left.\left\{\operatorname{id} \times p_{\sigma, \sigma}\right\}^{*} \mathcal{N}_{l_{\sigma, \sigma}}^{\text {ide }}\right|_{\mathcal{W}_{\sigma}^{\sigma}}=\left.\mathcal{N}_{j_{\sigma, \sigma}}^{\text {ide }}\right|_{\mathcal{W}_{\sigma}^{\sigma}} .
$$


We have thus verified the second part of the inductive assumption (I1) for $\sigma^{\prime}=\sigma$.

Since the immersions $\bar{\iota}_{\sigma, \sigma^{\prime}}$ with $\sigma^{\prime} \leq \sigma$ are embeddings by the inductive assumption ( $I 8$ ) in Section 4.3, so are the immersions

$$
\bar{j}_{\sigma, \sigma^{\prime}}:\left(\widetilde{\mathcal{M}}_{1,\left(I_{P}\left(\sigma^{\prime}\right), J_{P}\left(\sigma^{\prime}\right)\right)} \times \pi_{\varrho_{\sigma^{\prime}}(\sigma)}^{*} \pi_{\mathbb{P} F_{(\aleph, J)}}^{*} \mathcal{V}_{\left(\aleph_{B}\left(\sigma^{\prime}\right), J_{B}\left(\sigma^{\prime}\right)\right)}\right) / G_{\sigma^{\prime}} \longrightarrow \mathcal{V}_{1, \sigma^{\prime}}^{\sigma} \subset \mathcal{V}_{1, k}^{\sigma}
$$

induced by $j_{\sigma, \sigma^{\prime}}$. In particular, all of the morphisms

$$
\begin{aligned}
& \bar{j}_{\sigma_{\max }, \sigma^{\prime}}:\left(\widetilde{\mathcal{M}}_{1,\left(I_{P}\left(\sigma^{\prime}\right), J_{P}\left(\sigma^{\prime}\right)\right) \times \pi_{0,\left(\aleph_{B}\left(\sigma^{\prime}\right), J_{B}\left(\sigma^{\prime}\right)\right)}} \pi_{\mathbb{P} F_{(\aleph, J)}}^{*} \mathcal{V}_{\left(\aleph_{B}\left(\sigma^{\prime}\right), J_{B}\left(\sigma^{\prime}\right)\right)}\right) / G_{\sigma^{\prime}} \\
& \longrightarrow \mathcal{V}_{1, \sigma^{\prime}}^{\sigma_{\max }} \subset \mathcal{V}_{1, k}^{\sigma_{\max }}=\tilde{\pi}^{*} \overline{\mathfrak{M}}_{1, k}(\mathcal{L}, d)
\end{aligned}
$$

are embeddings. On the other hand, by the inductive assumption (I1),

$$
\widetilde{\mathcal{W}}_{\sigma^{\prime}} \equiv \mathcal{W}_{\sigma^{\prime}}^{\sigma_{\max }} \equiv j_{\sigma_{\max }, \sigma^{\prime}}^{-1}\left(\widetilde{\mathfrak{M}}_{1, k}^{0}(\mathcal{L}, d)\right) \equiv j_{\sigma_{\max }, \sigma^{\prime}}^{-1}\left(\mathcal{V}_{1,(0)}^{\sigma_{\max }}\right)
$$

is the closure of

$$
\begin{aligned}
\widetilde{\mathcal{M}}_{1,\left(I_{P}\left(\sigma^{\prime}\right), J_{P}\left(\sigma^{\prime}\right)\right)} \times & \left.\operatorname{ker} \widetilde{\mathfrak{D}}_{0}\right|_{\widetilde{\mathcal{Z}}_{\sigma^{\prime} ; B}^{0}} \\
& \subset \widetilde{\mathcal{M}}_{1,\left(I_{P}\left(\sigma^{\prime}\right), J_{P}\left(\sigma^{\prime}\right)\right)} \times \pi_{0,\left(\aleph_{B}\left(\sigma^{\prime}\right), J_{B}\left(\sigma^{\prime}\right)\right)}^{*} \pi_{\mathbb{P} F_{(\aleph, J)}}^{*} \mathcal{V}_{\left(\aleph_{B}\left(\sigma^{\prime}\right), J_{B}\left(\sigma^{\prime}\right)\right)} .
\end{aligned}
$$

By Proposition 5.5 and the inductive assumption (I8) in Section 4.3, this closure is

$$
\widetilde{\mathcal{M}}_{1,\left(I_{P}\left(\sigma^{\prime}\right), J_{P}\left(\sigma^{\prime}\right)\right)} \times\left.\operatorname{ker} \widetilde{\mathfrak{D}}_{\left(I_{P}\left(\sigma^{\prime}\right), J_{P}\left(\sigma^{\prime}\right)\right)}\right|_{\widetilde{\mathcal{Z}}_{\sigma^{\prime} ; B}},
$$

where $\quad \widetilde{\mathcal{Z}}_{\sigma^{\prime} ; B}=\widetilde{\mathcal{D}}_{\left(I_{P}\left(\sigma^{\prime}\right), J_{P}\left(\sigma^{\prime}\right)\right)}^{-1}(0)$.

Since the bundle section $\widetilde{\mathcal{D}}_{\left(I_{P}\left(\sigma^{\prime}\right), J_{P}\left(\sigma^{\prime}\right)\right)}$ is transverse to the zero set, $\widetilde{\mathcal{Z}}_{\sigma^{\prime} ; B}$ is a smooth subvariety of $\widetilde{\mathfrak{M}}_{0,\left(I_{P}\left(\sigma^{\prime}\right), J_{P}\left(\sigma^{\prime}\right)\right)}\left(\mathbb{P}^{n}, d\right)$ and

$$
\widetilde{\mathcal{W}}_{\sigma^{\prime}} \longrightarrow \widetilde{\mathcal{M}}_{1,\left(I_{P}\left(\sigma^{\prime}\right), J_{P}\left(\sigma^{\prime}\right)\right)} \times \widetilde{\mathcal{Z}}_{\sigma^{\prime} ; B}
$$

is a smooth vector bundle by Proposition 5.5. We conclude that

$$
\widetilde{\mathfrak{M}}_{1, k}^{0}(\mathcal{L}, d) \cap \mathcal{V}_{1, \sigma^{\prime}}^{\sigma_{\max }}
$$

is a smooth subvariety of $\widetilde{\mathfrak{M}}_{1, k}^{0}(\mathcal{L}, d)$ for all $\sigma^{\prime} \in \mathcal{A}_{1, k}(k, d)$. Its normal cone is a line bundle by the inductive assumption (I1). 


\section{Appendix A Most frequently used symbols and notation}

\begin{tabular}{|c|c|}
\hline$d$ & degree of stable maps to $\mathbb{P}^{n}$ \\
\hline$k$ & number of marked points for genus-one stable maps \\
\hline$n$ & dimension of projective space, $\mathbb{P}^{n}$ \\
\hline $\mathbb{C}, \mathbb{Q}$ & the sets of rational numbers, of complex numbers \\
\hline $\mathbb{Z}, \mathbb{Z}^{+}, \overline{\mathbb{Z}}^{+}$ & the sets of all, positive, nonnegative integers \\
\hline$[k]$ & $\{1, \ldots, k\}$ \\
\hline$\gamma_{\varrho}, \gamma_{(\mathcal{N}, J)}$ & tautological line bundles on $\mathbb{P} F_{\varrho}, \mathbb{P} F_{(\aleph, J)}$ \\
\hline$s_{i}$ & pairing of $L_{i}$ with $\mathbb{E}$ on $\overline{\mathcal{M}}_{1, I}$ and with $L_{0}$ on $\overline{\mathcal{M}}_{0,0 \sqcup I} ; \mathrm{p} 30$ \\
\hline$s_{\rho, i}$ & pairing of $L_{\rho, i}$ with $\mathbb{E}_{\rho}$ on $\overline{\mathcal{M}}_{1,(I, J)}^{\rho}, \rho \in \mathcal{A}_{1}(I, J) ; \mathrm{p} 33$ \\
\hline$\tilde{s}_{i}$ & nondegenerate pairing of $\widetilde{L}_{i}$ with $\widetilde{\mathbb{E}}$ on $\widetilde{\mathcal{M}}_{1,(I, J)} ; \mathrm{p} 33$ \\
\hline$s_{\rho,(l, i)}$ & pairing of $L_{\rho,(l, i)}$ with $\mathbb{E}_{\rho}$ on $\widetilde{\mathcal{M}}_{0, \varrho}^{\rho}, \rho \in \mathcal{A}_{0}(\varrho)$; Section 2.4 \\
\hline$\tilde{S}_{(l, i)}$ & nondegenerate pairing of $\widetilde{L}_{(l, i)}$ with $\widetilde{\mathbb{E}}$ on $\widetilde{\mathcal{M}}_{0, \varrho} ; \mathrm{p} 37$ \\
\hline $\mathcal{A}_{g}(I), \mathcal{A}_{g}(I, J)$ & collections of subvarieties of $\overline{\mathcal{M}}_{g, I}$, of $\overline{\mathcal{M}}_{g, I \sqcup J} ; \mathrm{p} 28, \mathrm{p} 31$ \\
\hline $\mathcal{A}_{0}(\varrho)$ & collection of subvarieties of $\mathbb{P} F_{\varrho}, \varrho=\left(I_{l}, J_{l}\right)_{l \in \mathfrak{N}} ;$ p 35 \\
\hline $\mathcal{A}_{g}(d, k)$ & collection of subvarieties of $\overline{\mathfrak{M}}_{g, k}\left(\mathbb{P}^{n}, d\right) ; \mathrm{p} 6, \mathrm{p} 40$ \\
\hline $\mathcal{A}_{0}(\aleph ; d, J)$ & collection of subvarieties of $\mathbb{P} F_{(\aleph, J)} ; \mathrm{p} 45$ \\
\hline $\mathcal{D}_{0}, \mathfrak{D}_{0}$ & "derivatives" of maps at the 0 -th marked point; $\mathrm{p} 43, \mathrm{p} 80$ \\
\hline $\mathcal{D}_{(\aleph, J)}, \mathfrak{D}_{(\aleph, J)}$ & "sums" of various $\mathcal{D}_{0}, \mathfrak{D}_{0} ; \mathrm{p} 48, \mathrm{p} 81$ \\
\hline$\widetilde{\mathcal{D}}_{\varrho}, \widetilde{\mathfrak{D}}_{\varrho}$ & induced derivatives over $\widetilde{\mathfrak{M}}_{(\aleph, J)}^{\varrho} ; \mathrm{p} 52, \mathrm{p} 82$ \\
\hline $\mathbb{E}$ & Hodge line bundle of holomorphic differentials on $\overline{\mathcal{M}}_{1, J}$ \\
\hline $\mathbb{E}_{\rho}, \widetilde{\mathbb{E}}$ & $\begin{array}{l}\text { line bundles on } \overline{\mathcal{M}}_{1,(I, J)}^{\rho}, \widetilde{\mathcal{M}}_{1,(I, J)}, \rho \in \mathcal{A}_{1}(I, J), \text { or } \widetilde{\mathcal{M}}_{0, \varrho}^{\rho}, \\
\widetilde{\mathcal{M}}_{0, \varrho}, \rho \in \mathcal{A}_{1}(\varrho) \text {, or } \mathfrak{M}_{0,(\aleph, J)}^{\rho}, \widetilde{\mathfrak{M}}_{0,(\boldsymbol{\aleph}, J)}\left(\mathbb{P}^{n}, d\right), \rho \in \mathcal{A}_{1}(\boldsymbol{\aleph} ; d, J), \\
\text { obtained from } \mathbb{E} \text {, or } \gamma_{\varrho}, \text { or } \gamma_{(\boldsymbol{\aleph}, J)} ; \mathrm{p} 32, \mathrm{p} 37, \mathrm{p} 52\end{array}$ \\
\hline$F_{\varrho}, F_{(\aleph, J)}$ & vector bundles over $\overline{\mathcal{M}}_{0, \varrho}, \overline{\mathfrak{M}}_{0,(\aleph, J)}\left(\mathbb{P}^{n}, d\right) ; \mathrm{p} 35, \mathrm{p} 44$ \\
\hline$F_{\varrho ; P}, F_{\varrho ; S}$ & subbundles of $F_{(\aleph, J)}, \varrho \in \mathcal{A}_{0}(\aleph ; d, J) ; \mathrm{p} 46$ \\
\hline$G_{\sigma}$ & symmetry group of the immersions $\iota_{\sigma}, \iota_{\sigma^{*}, \sigma} ; \mathrm{p} 10$ \\
\hline $\operatorname{Im}^{s} \iota$ & singular locus of immersion $\iota ; \mathrm{p} 38$ \\
\hline$L_{j}$ & $\begin{array}{l}\text { universal tangent line bundle for } j \text {-th marked point over } \overline{\mathcal{M}}_{g, J} \\
\text { or } \overline{\mathfrak{M}}_{g, J}\left(\mathbb{P}^{n}, d\right), j \in J\end{array}$ \\
\hline$L_{\rho, i}, \tilde{L}_{i}$ & line bundles on $\overline{\mathcal{M}}_{1,(I, J)}^{\rho}, \widetilde{\mathcal{M}}_{1,(I, J)}, \rho \in \mathcal{A}_{1}((I, J) ; \mathrm{p} 32$ \\
\hline$L_{\rho,(l, i)}, \widetilde{L}_{(l, i)}$ & line bundles on $\widetilde{\mathcal{M}}_{0, \varrho}^{\rho}, \widetilde{\mathcal{M}}_{0, \varrho}, \rho \in \mathcal{A}_{0}(\varrho) ; \mathrm{p} 37$ \\
\hline $\mathbb{L}$ & universal tangent line bundle on $\widetilde{\mathcal{M}}_{1,(I, J)}, \widetilde{\mathcal{M}}_{0, \varrho} ; \mathrm{p} 31, \mathrm{p} 35$ \\
\hline & the line bundle associated to the sheaf $\mathcal{O}_{\mathbb{P}^{n}}(a), a>0$ \\
\hline
\end{tabular}




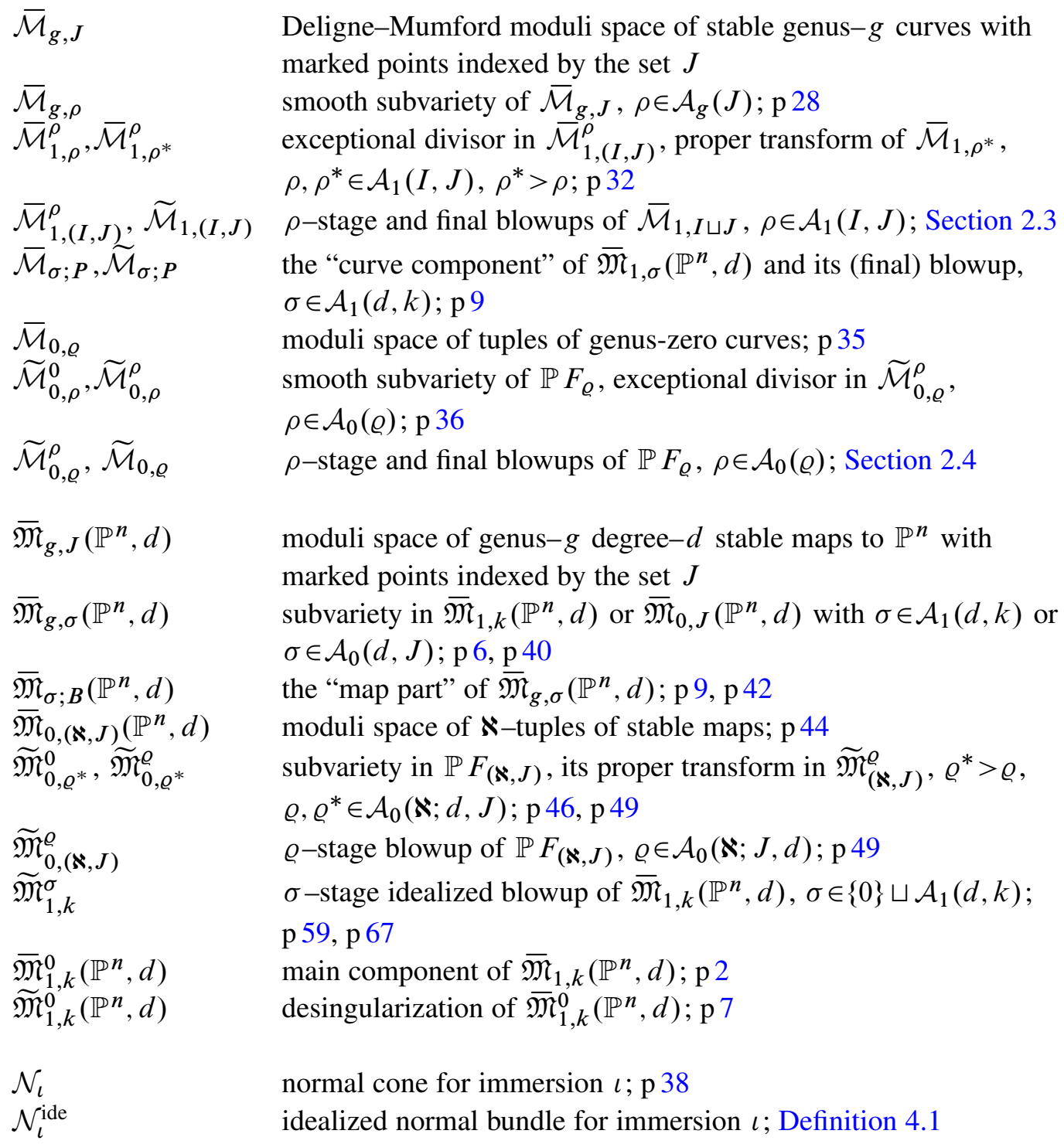

\section{References}

[1] MF Atiyah, R Bott, The moment map and equivariant cohomology, Topology 23 (1984) 1-28 MR721448

[2] A Beauville, Quantum cohomology of complete intersections, Mat. Fiz. Anal. Geom. 2 (1995) 384-398 MR1484335 
[3] M Bershadsky, S Cecotti, H Ooguri, C Vafa, Holomorphic anomalies in topological field theories, Nuclear Phys. B 405 (1993) 279-304 MR1240687

[4] A Bertram, Another way to enumerate rational curves with torus actions, Invent. Math. 142 (2000) 487-512 MR1804158

[5] C Fontanari, Towards the cohomology of moduli spaces of higher genus stable maps arXiv: math.AG/0611754

[6] W Fulton, R Pandharipande, Notes on stable maps and quantum cohomology, from: "Algebraic geometry—Santa Cruz 1995", Proc. Sympos. Pure Math. 62, Amer. Math. Soc., Providence, RI (1997) 45-96 MR1492534

[7] A Gathmann, Absolute and relative Gromov-Witten invariants of very ample hypersurfaces, Duke Math. J. 115 (2002) 171-203 MR1944571

[8] A Givental, The mirror formula for quintic threefolds, from: "Northern California Symplectic Geometry Seminar", Amer. Math. Soc. Transl. Ser. 2 196, Amer. Math. Soc., Providence, RI (1999) 49-62 MR1736213

[9] M Gromov, Pseudoholomorphic curves in symplectic manifolds, Invent. Math. 82 (1985) 307-347 MR809718

[10] K Hori, S Katz, A Klemm, R Pandharipande, R Thomas, C Vafa, R Vakil, E Zaslow, Mirror symmetry, Clay Mathematics Monographs 1, American Mathematical Society, Providence, RI (2003) MR2003030With a preface by Vafa

[11] M Kontsevich, Y Manin, Gromov-Witten classes, quantum cohomology, and enumerative geometry, Comm. Math. Phys. 164 (1994) 525-562 MR1291244

[12] Y-P Lee, Quantum Lefschetz, hyperplane theorem, Invent. Math. 145 (2001) 121-149 MR1839288

[13] J Li, A Zinger, On the genus-one Gromov-Witten invariants of complete intersections arXiv:math.AG/0507104

[14] B H Lian, K Liu, S-T Yau, Mirror principle. I, Asian J. Math. 1 (1997) 729-763 MR1621573

[15] D Maulik, R Pandharipande, A topological view of Gromov-Witten theory, Topology 45 (2006) 887-918 MR2248516

[16] R Pandharipande, Intersections of $\mathbf{Q}$-divisors on Kontsevich's moduli space $\bar{M}_{0, n}\left(\mathbf{P}^{r}, d\right)$ and enumerative geometry, Trans. Amer. Math. Soc. 351 (1999) 14811505 MR1407707

[17] Y Ruan, G Tian, A mathematical theory of quantum cohomology, J. Differential Geom. 42 (1995) 259-367 MR1366548

[18] R Vakil, The enumerative geometry of rational and elliptic curves in projective space, J. Reine Angew. Math. 529 (2000) 101-153 MR1799935 
[19] R Vakil, Murphy's law in algebraic geometry: badly-behaved deformation spaces, Invent. Math. 164 (2006) 569-590 MR2227692

[20] R Vakil, A Zinger, A natural smooth compactification of the space of elliptic curves in projective space, Electron. Res. Announc. Amer. Math. Soc. 13 (2007) 53-59 MR2320682

[21] A Zinger, Enumeration of genus-two curves with a fixed complex structure in $\mathbb{P}^{2}$ and $\mathbb{P}^{3}$, J. Differential Geom. 65 (2003) 341-467 MR2064428

[22] A Zinger, Enumeration of one-nodal rational curves in projective spaces, Topology 43 (2004) 793-829 MR2061208

[23] A Zinger, Counting rational curves of arbitrary shape in projective spaces, Geom. Topol. 9 (2005) 571-697 MR2140990

[24] A Zinger, A sharp compactness theorem for genus-one pseudo-holomorphic maps arXiv:math.SG/0406103

[25] A Zinger, On the structure of certain natural cones over moduli spaces of genus-one holomorphic maps, Adv. Math. 214 (2007) 878-933 MR2349722

[26] A Zinger, Reduced genus-one Gromov-Witten invariants arXiv:math.SG/0507103

[27] A Zinger, Intersections of tautological classes on blowups of moduli spaces of genusone curves, Michigan Math. J. 55 (2007) 535-560 arXiv:math. AG/0603357

[28] A Zinger, The reduced genus-one Gromov-Witten invariants of Calabi-Yau hypersurfaces arXiv:math/0705.2397

Department of Mathematics, Stanford University

Stanford, CA 94305-2125, USA

Department of Mathematics, SUNY Stony Brook

Stony Brook, NY 11794-3651, USA

vakil@math.stanford.edu, azinger@math.sunysb.edu

http://math.stanford.edu/ vakil, http://www.math.sunysb.edu/ azinger

Proposed: Jim Bryan

Received: 4 March 2007

Seconded: Gang Tian, Eleny Ionel

Revised: 12 October 2007 